\title{
A Constraint-Based Approach to \\ Manipulator Kinematics and Singularities
}

\author{
Seyedvahid Amirinezhad \\ VICTORIA UNIVERSITY OF WELLINGTON \\ Te Whare Wānanga o te Ūpoko o te Ika a Māui

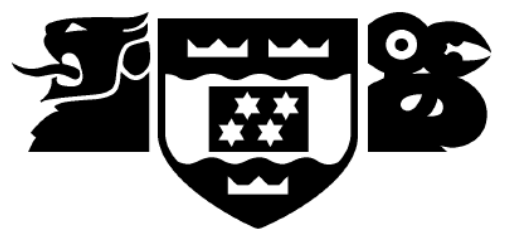 \\ School of Mathematics and Statistics \\ Te Kura Mātai Tatauranga
}

\begin{abstract}
A thesis
submitted to the Victoria University of Wellington in fulfilment of the requirements for the degree of

Doctor of Philosophy

in Mathematics.

Victoria University of Wellington
\end{abstract}

2019 



\begin{abstract}
In this thesis, a differential-geometric approach to the kinematics of multibody mechanisms is introduced that enables analysis of singularities of both serial and parallel manipulators in a flexible and complete way. Existing approaches such as those of Gosselin and Angeles [1], Zlatanov et al. [2] and Park and Kim [3] make use of a combination of joint freedoms and constraints and so build in assumptions. In contrast, this new approach is solely constraint-based, avoiding some of the shortcomings of these earlier theories.

The proposed representation has two core ingredients. First, it avoids direct reference to the choice of inputs and their associated joint freedoms and instead focuses on a kinematic constraint map (KCM), defined by the constraints imposed by all joints and not requiring consideration of closure conditions arising from closed loops in the design. The KCM is expressed in terms of pose (i.e. position and orientation) variables, which are the coordinates of all the manipulator's links with respect to a reference frame. The kinematics of a given manipulator can be described by means of this representation, locally and globally. Also, for a family of manipulators defined by a specific architecture, the KCM will tell us how the choice of design parameters (e.g. link lengths) affects these kinematic properties within the family.
\end{abstract}

At a global level, the KCM determines a subset in the space of all pose variables, known as the configuration space (C-space) of the manipulator, whose topology may vary across the set of design parameters. The Jacobian (matrix of first-order partial derivatives) of the KCM may become singular 
at some specific choices of pose variables. These conditions express a subset called the singular set of the C-space. It is shown that if a family of manipulators, parametrised by a manifold $\mathbb{R}^{d}$ of design parameters, is "wellbehaved" then the pose variables can be eliminated from the KCM equations together with the conditions for singularities, to give conditions in terms of design parameters, that define a hypersurface in $\mathbb{R}^{d}$ of manipulators in the class that exhibit C-space singularities. These are referred to as Grashof-type conditions, as they generalise classically known inequalities classifying planar 4-bar mechanisms due to Grashof [4].

Secondly, we develop the theory to incorporate actuator space (A-space) and workspace (W-space), based on a choice of actuated joints or inputs and on the manipulator's end-effector workspace or outputs. This will facilitate us with a framework for analysing singularities for forward and inverse kinematics via input and output mappings defined on the manipulator's C-space. This provides new insight into the structure of the forward and inverse kinematics, especially for parallel manipulators.

The theory is illustrated by a number of applications, some of which recapitulate classical or known results and some of which are new. 


\section{Acknowledgements}

Before everything else, I would like to express gratitude to my thesis supervisor, A/Prof. Peter Donelan - the former head of School of Mathematics and Statistics (SMS) at Victoria University of Wellington (VUW), for his indispensable help and guidance during the last few years. He has devoted a lot of his time and energy for keeping me on a right and clear research pathway. He has been always around like a friend to support me in difficulties and to celebrate good times.

It has been a unique opportunity to complete my $\mathrm{PhD}$ degree in the SMS at VUW. I would like to express my appreciation to the university for providing me with a fabulous academic environment to peacefully conduct my research. In addition, I thank the technical staff for ensuring all technical requirements and the administrative staff for keeping the atmosphere warm and friendly.

I am genuinely thankful to Prof. Andreas Müller - the director of the Institute of Robotics at Johannes Kepler University Linz (JKU) - for his valuable friendship and collaboration. He has kindly invited me to be a research visitor for a few months at JKU. This was a great opportunity and special experience for me to collaborate with him and his research team.

Last but not least, I want to sincerely thank my parents and my sister for their endless and inexhaustible support in my life. Their encouragement and companionship motivated me to commence this journey exploring the world and science. 


\section{Contents}

Abstract ............................ ii

Acknowledgements ................... ii

Contents ....................................

List of Figures . . . . . . . . . . . . . . . . . . xi

1 Introduction 1

1.1 Kinematic geometry . . . . . . . . . . . . . . . 1

1.2 Kinematic singularities . . . . . . . . . . . . . . 4

1.3 Literature review . . . . . . . . . . . . . . . 6

1.4 Further discussions . . . . . . . . . . . . . . . . . . . 8

1.5 A constraint-based approach . . . . . . . . . . . . 14

1.6 Thesis structure . . . . . . . . . . . . . . . 16

$\begin{array}{llr}2 & \text { Euclidean group } & 19\end{array}$

2.1 Lie group . . . . . . . . . . . . . . . . . . . . . 19

2.2 Euclidean groups . . . . . . . . . . . . . . . . . . 22

2.3 Coordinate frames and pose variables . . . . . . . . . 27

2.4 Group parametrisations . . . . . . . . . . . . . 28

2.4.1 Special orthogonal groups . . . . . . . . . . . . 28

2.4.2 Quaternions and spatial rotations . . . . . . . . 30

2.4.3 The special Euclidean groups . . . . . . . . . . . 34

2.4.4 Dual numbers . . . . . . . . . . . . . . 36

2.4.5 Dual quaternions and spatial displacement . . . . . . 37 
2.5 The pose space . . . . . . . . . . . . . . . . . . . 41

3 The Euclidean Lie algebra \& associated geometry 45

3.1 Tangent spaces \& Lie algebras . . . . . . . . . . . . . . . 46

3.2 Lie algebra of $S O(3) \ldots \ldots \ldots$. . . . . . . . . . . . . 47

3.3 Lie algebra of $S E(3) \ldots \ldots \ldots \ldots$

3.3.1 Adjoint representation . . . . . . . . . . . . 52

3.4 Line geometry . . . . . . . . . . . . . . . . 54

3.4.1 Lines in $\mathbb{R}^{3} \ldots \ldots \ldots \ldots \ldots \ldots$

3.4.2 Plücker coordinates . . . . . . . . . . . 56

3.4.3 Screw theory . . . . . . . . . . . . 58

3.5 The exponential map and 1-parameter

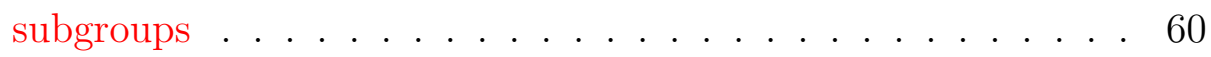

3.6 Infinitesimal displacements and twists in the pose space . . . . 61

4 Constraints versus freedoms $\quad 65$

4.1 Chebyshev-Grübler-Kutzbach formula . . . . . . . . . . 67

4.2 Joint constraint equations . . . . . . . . . . . . . . . 68

4.3 Planar kinematic pairs . . . . . . . . . . . . . . 72

4.3.1 R-joint constraints . . . . . . . . . . . 73

4.3.2 P-joint constraints . . . . . . . . . . . . . 74

4.3 .3 2P-joint constraints . . . . . . . . . . 77

4.4 Spatial kinematic pairs . . . . . . . . . . . . . . 78

4.4.1 R-joint constraints . . . . . . . . . . . . 79

4.4.2 P-joint constraints . . . . . . . . . . . . 82

4.4.3 H-joint constraints . . . . . . . . . . . . . 84

4.4.4 C-joint constraints . . . . . . . . . . . 84

4.4.5 S-joint constraints . . . . . . . . . . 85

4.4 .6 E-joint constraints . . . . . . . . . . . 86

4.4 .7 U-joint constraints . . . . . . . . . . . . 88 
4.5 Kinematic constraint mapping . . . . . . . . . . . . . . 90

4.6 Joint variables . . . . . . . . . . . . . . . . . . . . . . . . . . 92

4.6.1 Planar joint variables . . . . . . . . . . . . . . . . 92

4.6.2 Spatial joint variables . . . . . . . . . . . . . . 93

4.7 Actuator space and workspace - input and output maps . . . . 94

$\begin{array}{llr}5 & \text { Singularities } & 99\end{array}$

5.1 Singularities of mappings . . . . . . . . . . . . . . . . . . . . 99

$5.2 \quad$ C-space singularities . . . . . . . . . . . . . . . 103

5.2 .1 Design parameters . . . . . . . . . . . . . 103

5.2 .2 Transversality . . . . . . . . . . . . . . . . . . 104

5.2.3 Grashof-type conditions . . . . . . . . . . 106

5.3 Input and output singularities . . . . . . . . . . . . . . . . 108

5.3 .1 Ceva's theorem ................... 111

6 Classical planar mechanisms $\quad 115$

6.1 Planar 4-R linkage . . . . . . . . . . . . . . . . . 116

6.1.1 C-space singularities and Grashof-type conditions . . . 118

6.1.2 Input mapping and singularities . . . . . . . . . . . . 121

6.1.3 Output mapping and singularities . . . . . . . . . 122

6.2 Planar RRRP linkage . . . . . . . . . . . . . . . . . . . . 125

6.2.1 C-space singularities and Grashof-type conditions . . . 126

6.2.2 Input mapping and singularities . . . . . . . . . . 128

6.2.3 Output mapping and singularities . . . . . . . . . . . . 128

6.3 Extended Watt planar linkage . . . . . . . . . . . . . 130

7 Planar parallel manipulators 135

7.1 Planar 3-RRR parallel manipulator . . . . . . . . . . . . . 135

7.1.1 C-space singularities and Grashof-type conditions . . . 137

7.1.2 Output mapping and singularities . . . . . . . . . . 140

7.1.3 Input mapping and singularities . . . . . . . . . . . . 142 
7.2 Planar 3-RPR parallel manipulator . . . . . . . . . . . 145

7.2.1 Corank 1 singularities . . . . . . . . . . . 147

7.2.2 Corank 2 singularities . . . . . . . . . . . . . . 149

7.2.3 Output mapping and singularities . . . . . . . . . . . 149

7.2.4 Input mapping and singularities . . . . . . . . . . 151

8 Planar geared manipulators $\quad 155$

8.1 The mechanism . . . . . . . . . . . . . . . 156

8.2 Input-output analysis via the matroid method . . . . . . . . 158

$8.3 \mathrm{KCM}$ and C-space singularities . . . . . . . . . . . . 163

8.4 Forward kinematic and singularity analysis . . . . . . . . . 167

9 Spatial parallel manipulators $\quad 171$

9.1 Spatial 3-RPS parallel manipulator . . . . . . . . . . . . 172

10 Conclusion $\quad 189$

A Constraint equations for spatial joints 193

B Singularities and Grashof-type conditions

$\begin{array}{ll}\text { for } 3-R R R ~ P P M & 201\end{array}$

$\begin{array}{ll}\text { References } & 204\end{array}$ 


\section{List of Figures}

3.1 Lines in 3 -space . . . . . . . . . . . . . . . . . . 55

4.1 Adjacent links constrained at a planar R-joint . . . . . . . . 73

4.2 Adjacent links constrained at a planar P-joint: a) RPR-chain and b) RPP-chain . . . . . . . . . . . . . . . . . 75

4.3 Adjacent links constrained at a spatial R-joint . . . . . . . . 80

4.4 Adjacent links constrained at a spatial P-joint . . . . . . . . 82

4.5 Adjacent links constrained at a S-joint . . . . . . . . . . 85

4.6 Adjacent links constrained at an E-joint . . . . . . . . . 87

4.7 Adjacent links constrained at a U-joint . . . . . . . . . . 88

4.8 Planar joint variables . . . . . . . . . . . . . . . . . . . 93

4.9 Kinematic mappings and the C-space . . . . . . . . . . 96

5.1 An implicit function with associated projections . . . . . . . 102

5.2 Forward and inverse kinematic mappings . . . . . . . . . . . 109

5.3 A triangle considered for Ceva's theorem . . . . . . . . . . 112

6.1 4-R linkage with associated frames and design parameters . . 116

6.2 A C-space singularity for 4-R linkage . . . . . . . . . . . . 119

6.3 A shaky (immobile) configuration for 4-R linkage . . . . . . 121

6.4 An input singularity for 4-R linkage . . . . . . . . . . . . 122

6.5 An output singularity corresponding to $L_{3}$ for 4 -R linkage . . 123

6.6 An output singularity corresponding to $L_{2}$ for 4 -R linkage . . 124 
6.7 3RP linkage with associated moving frames and design param-

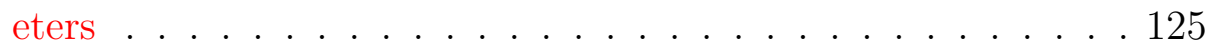

6.8 A C-space singularity for RRRP linkage . . . . . . . . . . 126

6.9 An input singularity for RRRP linkage . . . . . . . . . . . 127

6.10 An output singularity corresponding to $L_{3}$ for RRRP linkage . 128

6.11 An output singularity corresponding to $L_{2}$ for RRRP linkage . 129

6.12 Extended Watt 7-R linkage with associated moving frames and design parameters . . . . . . . . . . . . 130

6.13 A C-space singularity associated to the 4-R at a flat configuration132

6.14 A flat configuration corresponding to the C-space singularity . 133

7.1 3-RRR PPM with associated moving frames and design pa-

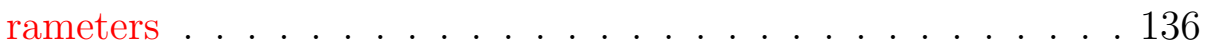

7.2 C-space singularities for $3-\mathrm{RRR}$ PM . . . . . . . . . . . . 138

7.3 Special C-space singularities for $3-\mathrm{RRR}$ PPM . . . . . . . . . . . 140

7.4 3-RRR PPM output singularities . . . . . . . . . . . . . . . 141

7.53 -RRR PPM input singularities . . . . . . . . . . . . . . . . 142

7.6 Special input singularities for $3-\underline{R R R ~ P P M ~ . ~ . ~ . ~ . ~ . ~ . ~ . ~ . ~ . ~ . ~} 144$

7.7 3-RR PPM input singularities . . . . . . . . . . . . . . 145

7.8 3-RPR PPM with associated moving frames and design parameters ........................ 146

7.9 Corank 1 singular configurations for 3-RPR PPM . . . . . . 148

7.10 Corank 2 singular coonfigurations for 3-RPR PPM ... . 149

7.11 3-RPR PPM output singularities . . . . . . . . . . . 150

7.12 3-RPR PPM input singularities . . . . . . . . . . . . . 151

7.13 A special input singularity for 3-RPR PPM . . . . . . . . . . 152

7.14 3-RPR input singularities . . . . . . . . . . . . . . 153

8.1 3-dof geared planar manipulator . . . . . . . . . . . . 156

8.2 Functional schematic of the manipulator in Figure 8.1 . . . . 157 
8.3 Associated digraph . . . . . . . . . . . . . . . . . . 158

8.4 Planar GM with associated frames and links' lengths . . . . . 164

9.1 3-RPS spatial PM with associated moving frames and design

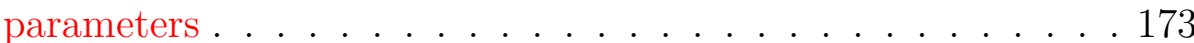




\section{Chapter 1}

\section{Introduction}

In this thesis, we aim to introduce a robust approach that can be employed to fully understand the kinematics of any serial or parallel manipulator. In particular, the main problem which we are going to address is identifying singularities of parallel manipulators (PMs) to which there is a lack of a comprehensive approach. Understanding singularities of manipulators has been one of the major challenges of robot kinematics for more than 35 years. While there is now detailed understanding of singularities for many specific robot architectures and a number of approaches to analysing and classifying singularities in general, there is not a robust mathematical model underpinning these.

\subsection{Kinematic geometry}

While the full mathematical study of manipulators and robot systems involves statics, dynamics, control, sensing, environment and more, underpinning all these is understanding their kinematics. Kinematics concern the motion capabilities of the system, without reference to forces, but may include velocity and acceleration analysis. We make the assumption that manipulators and robot systems comprise rigid components (i.e. links) connected 
by a variety of joints. Therefore, the basic requirement is a mathematical description of the placement and movement of rigid bodies.

Kinematic geometry has a long history motivated by a desire to understand the motion of, on the one hand, individual objects such as the planets and projectiles, but on the other by machines and mechanisms combining several components. The term kinematics is due to Ampère [5, 6], though the conceptual history of the subject is much older. In the industrial age kinematics evolved rapidly, as the development of machines as a means of converting motion became increasingly important. Advances in kinematics in the $19^{\text {th }}$ century were linked very closely with new ideas in geometry. Hamilton's discovery of the quaternions [7] was motivated precisely by the desire to describe spatial motion in an effective way and this then led to the development of linear algebra. Grassmann, Cayley, Clifford [8, 9] and others discovered further algebraic ways, including dual quaternions, for describing geometric motion. By the beginning of the $20^{t h}$ century, Ball had set out the foundations of screw theory [10] and its application to statics, and Study [11] had significantly advanced the study and application of dual quaternions.

The advance of robotics in the second half of the $20^{\text {th }}$ century led to new questions in kinematics, in particular about modelling systems with multiple degrees of freedom (dofs), in contrast to the predominantly 1-dof models required for mechanisms. Several important distinctions arose in the modelling. Robot systems were characterized primarily as either serial or parallel. A serial manipulator or robot consists of an open chain of rigid links with successive pairs connected by 1-dof joints. Typically, the purpose of a serial manipulator is to convert inputs at the joints to motion of the final link (i.e. end-effector). On the other hand, in a parallel manipulator there are closed chains of links. Typical architectures for parallel manipulators involve a number of serial chain legs connecting a base to a platform. The existence of closed loops means that the number of joints exceeds the required 
dof(s) for the platform, so that only a subset of joints are required as inputs, while other joints move passively. Moreover, the passive joints may have greater than one dof, for example they may be universal (2-dof) joints or ball-and-socket (3-dof) joints.

The description of serial and parallel manipulators also makes clear a distinction between inputs - typically thought of as joint variables - and outputs which are typically the pose (i.e. position and orientation) or configuration of the end-effector or platform. This distinction gives rise to what are usually regarded as the fundamental problems of robot kinematics, namely the forward and inverse kinematic problems (FKP, IKP respectively). The FKP concerns locating the output of the system for a given input, while the IKP requires determining how to choose the input to realise a given output. It is not clear that either of these problems has a well-defined or unique solution. Generally, however, it is the case that the FKP is relatively straightforward and well-defined for serial manipulators, while the IKP is more easily solved for parallel manipulators. As a consequence, emphasis in the robot kinematics research has been placed on the serial IKP and the parallel FKP. Important in the kinematics of both classes of mechanisms is the Jacobian matrix, originally thought of in robotics as the instantaneous linear relation between input and output velocities.

Early advances in solving the IKP for serial manipulators was made by Peiper [12]. An important tool in the analysis and design of serial manipulators was the formulation of a matrix description due to Denavit and Hartenberg [13]. Since serial manipulators were far more widely used in industry, the mathematical development of their kinematics was earlier than that for parallel manipulators. While, the key prototype for parallel mechanisms, independently developed in quite different settings by Gough and Stewart [14, 15] does have quite a long history, the mathematical descriptions of such mechanisms have been more recent. Hunt [16] and Phillips [17] 
demonstrated that Ball's screw theory was an essential tool. Line geometry [18] and algebraic geometry, especially modern computational techniques like Gröbner base [19], also became important. By the early $21^{\text {st }}$ century it was clear that the kinematic geometry of parallel mechanisms is more complicated than allowed for by earlier mathematical models - in particular, the role of passive joints cannot be overlooked. It also had become clear that for all types of mechanisms and robot systems, it is essential to understand and analyse kinematic singularities.

\subsection{Kinematic singularities}

A singularity of a manipulator is usually interpreted physically as a configuration where an instantaneous and uncontrollable change occurs in its motion and more specifically its dofs which is the number of independent motions that a manipulator is expected to have, either instantaneously or over a some finite interval. A sudden (unexpected) change can be either loss or gain of mobility.

The presence of singularities may result in dexterity issues caused by loss of mobility [20] or controllability issues caused by gain of mobility [21] in a manipulator. They also give rise to possible technical failures such as irresistible torques/forces on the mechanical components [22], loss of stiffness/compliance [23], and malfunctioning of control procedures [24].

Consequently, one of the crucial stages of designing a manipulator is analysis of singularities. Although it is mostly preferred to avoid singularities, existence of singularities in some architectures may result in some advantages such as a higher tolerance of extremely large external torques [25] and a smoother control procedure of the end-effector [26].

Mathematically, a singularity of manipulator is determined by a drop in rank of the Jacobian matrix of some mapping determining its kinematics. How to specify the mapping and what variables are considered are the main 
differences among various approaches [1, 27, 28].

A key goal of singularity analysis of a given manipulator architecture is to determine singular configurations and how they are influenced by choice of design parameters [29]. There may be certain geometric conditions relating the component links and joints of a manipulator, such as collinearity, coplanarity or symmetries, represented by equations on the design parameters, which assure existence of a singularity.

For a given manipulator with certain tasks to do, we may only need to examine some particular aspects of its singular behaviour. For example, we may wish to identify maximal singularity-free regions in its operational space; or we may want to design in specific singular behaviour. Ideally, this needs us to study the manipulator globally in order to capture relevant information on possible singular configurations.

This presents a serious challenge. Many approaches to singularity analysis establish that in a given configuration, a manipulator must have singular behaviour. However, establishing all such configurations is not addressed. Indeed, the fact that singularities are, in some sense, an instantaneous phenomenon suggests that a global approach is not necessary or even possible. This view is associated with the idea of that a Jacobian is simply a linear relationship between joint and input velocities and output (end-effector) velocities. While this is true, it may lose sight of the way the Jacobian varies as the manipulator moves and more significantly, exactly what the possible configurations of the manipulator are. These shortcomings become especially apparent for parallel manipulators where the total mobility may involve joints and links that are not directly part of the input nor the output, but are "passive".

In order to give a more complete approach to singularity analysis for manipulators, we return to basics and attempt to build a coherent kinematics model that can capture all aspects. 


\subsection{Literature review}

Here is a brief review of the literature on kinematic singularities. To the best of author's knowledge, the idea of singularity analysis of mechanisms and manipulators dates back to the late 1960s when Whitney [30] identified singularities when he was trying to develop a control algorithm for manipulators and prostheses. He proposed a method which is equivalent to the pseudo-inverse of a singular Jacobian matrix. In the late 70s, Hunt [16] defined uncertainty configurations for PMs where the mechanism has an instantaneous increase in mobility.

Since then, the research literature has divided to mainly concentrate on either serial or on parallel manipulators, rather than both. This review focuses on research that addresses general issues of kinematics modelling and singularity analysis, rather than on specific manipulators.

In respect of PMs, Merlet [18] employed Grassmann line geometry in order to explain singularities. Shortly after that, Gosselin and Angeles [1] introduced a new approach to singularities of PMs. Their approach was based on Jacobian analysis of an implicit kinematic formulation $F(\theta, x)=$ 0 , where $\theta$ represents input joint variables and $x$ output variables. It was assumed that there are equal numbers of these. Their approach facilitated understanding of PM singularities, and they introduced a simple classification of singularities. Their approach is widely cited and underpins much of the subsequent singularity analysis for PMs.

Several researchers have concentrated on mathematical aspects and used singularity theory [31] to understand singularities of manipulators. Pai and Leu [32] proposed the notion of a generic kinematic map for serial manipulators, whose singular sets form smooth manifolds of prescribed dimension in the joint space of the manipulator. This included consideration of secondorder derivatives of the forward mapping to formalise the so-called transversality conditions [33]. Tchoń and Muszyński [34, 35] explicitly introduced 
an approach, which comes from the singularity theory of maps, to provide normal forms of kinematic mappings. Although this approach was meant to focus on kinematic singularities, it was also acknowledged that there are special forms that significantly limit the so-called genericity of a serial manipulator.

Gibson, Donelan et al. [36, 37, 38, 39] analysed the relation between a forward kinematic mapping and the singularities of trajectories based on singularity-theoretic terms. In a review of singularity-theoretic methods with application to kinematics, Donelan [29] emphasised the importance of transversality as a tool for establishing genericity.

Burdick [40] proposed a geometric factorisation of the singularity set in order to classify generic and non-generic cases in the class of spatial 3R manipulators. In this context, Wenger et al. [41, 42] introduced the idea of cuspidal manipulators which can change their posture without crossing a singular set. Karger [43] explicitly used Lie group and Lie algebra structure to develop a theory which allows to describe higher order singularities for serial manipulators.

Lerbet [44] introduced concepts of analytic geometry, such as the tangent cone, to provide an intrinsic analysis of singularities for both open and closed chain mechanisms. Based on Lie group theory, he described the structure of the configuration space whether or not it has singularities. Lerbet and Hao [45] also explicitly utilised product of exponentials (PoEs) [46] and transversality in order to perform singularity analysis up to the second order.

By using algebraic geometry, Gibson et al. [47, 48] established a methodology to analyse the topology and singularities for planar mechanisms. Karger and Husty [49] also used algebraic equations to define spatial kinematics and for classification of self-motions, which are finite branch of motions when actuated joints are static. Torras et al. [50], Ben-Horin and Shoham [51] have approached kinematics and singularities of parallel manipulators by using 
geometric algebra.

Returning to the underlying kinematic model of Gosselin and Angeles for PMs, Zlatanov et al. [2] noticed the importance of passive joints and refined the classification of singularities for a general mechanism based on instantaneous kinematics into six types based on various combinations of ways in which the rank of a Jacobian matrix, involving input, output and passive joint variables, drops below its expected value. This approach has more recently been revised by Müller [52] and Bohigas et al. [53] so that the input map is treated as a function from the configuration space.

Park and Kim [54] proposed a differential geometric framework to stratify kinematic singularities into three types - namely configuration space singularities, actuator singularities, and end-effector singularities. Zlatanov et al. [55] re-examined singularities of PMs in more details and introduced the idea of configuration space singularities where a passive switching between different motion modes is possible. This is also considered by Shvalb et al. [56], as topological singularities. Chen [57] emphasised the difference between the global mobility and local mobilities with different orders and revealed the corresponding relations in between.

Most recently, Müller [58] has returned to the concept of genericity of a kinematic mapping - the likeliness of singularities to happen and their stability. He discussed whether singularities of a kinematic mapping comprise smooth manifolds or not by examining two genericity concepts. He also revisited the kinematic tangent cone [59, 60], as an approximation of the C-space, and used it for identification of singularities by analysing the local geometry of the set of critical points.

\subsection{Further discussions}

In this section, we provide a more detailed description of the research dis-

cussed briefly in the literature review that has had the most influence on 
developing the robust approach we propose for analysis and understanding of PM singularities. The analysis for singularities of serial manipulators is in many ways more straightforward, but does not lend itself to generalisation to PMs. Whereas, our proposed approach is motivated by consideration of PMs based on the the ideas addressed in this section, and therefore it easily works also for serial manipulators.

Gosselin and Angeles [1] laid the foundations for most of the subsequent work on PM singularities. They proposed an approach which begins by selecting input coordinates $\boldsymbol{\theta}$ and output coordinate $\mathbf{x}$, where $\boldsymbol{\theta}$ and $\mathbf{x}$ are assumed to be of the same dimension and equal to the global mobility $\mu$ of the mechanism. A relationship between the input (the actuated joint variables) and the output (the pose variables of the end-effector) is then described by an implicit function $F(\boldsymbol{\theta}, \mathbf{x})=0$. Typically, this equation is assumed to arise from some closure conditions on the manipulator.

Taking time derivatives of $F$ and applying the chain rule yields

$$
\left(D_{\mathbf{x}} F\right) \dot{\mathbf{x}}+\left(D_{\boldsymbol{\theta}} F\right) \dot{\boldsymbol{\theta}}=0
$$

where $D_{\mathbf{x}} F$ and $D_{\boldsymbol{\theta}} F$ denote the matrices of partial derivatives of $F$ with respect to $\mathbf{x}$ and $\boldsymbol{\theta}$, respectively. Based on this approach, there are three basics types of singularities: (i) if $\operatorname{det}\left(D_{\boldsymbol{\theta}} F\right)=0$, ii) if $\operatorname{det}\left(D_{\mathbf{x}} F\right)=0$, and iii) if $\operatorname{det}\left(D_{\boldsymbol{\theta}} F\right)=\operatorname{det}\left(D_{\mathbf{x}} F\right)=0$. In case (i), equation (1.1) can be satisfied by some non-zero $\dot{\boldsymbol{\theta}}$ with $\dot{\mathrm{x}}=0$ the end-effector loses a dof. On the other hand, (ii) allows a non-zero $\dot{\mathbf{x}}$ with $\dot{\boldsymbol{\theta}}=0$ so the end-effector is instantaneously mobile with the actuators locked. Type (iii) involves a combination of these and may be related to a configuration space singularities, which we discuss in Chapter 5. This typically requires conditions on design parameters as observed in examples in [1].

Zlatanov et al. [2] developed a methodology based on the study of the instantaneous kinematics. They noted that for the purposes of passive joints, 
this becomes especially important for PMs with limited dofs (i.e. less than six for a spatial mechanism). The $n$ active joint velocities are referred to as the input and a set of $n$ velocities, which specify the instantaneous motion of the end-effector, as the output. The output equation determines the output $T$ in terms of the input $\Omega_{a}$ and the $N-n$ passive joint velocities $\Omega_{p}$ by $T=A(q)\left[\Omega_{a}^{T}, \Omega_{p}^{T}\right]^{T}$, where $A(q)$ is an $n \times n$ Jacobian matrix and $q$ uniquely identifies the configuration of the PM.

For a PM, joint velocities must also satisfy the loop-closure equations $D(q)\left[\Omega_{a}^{T}, \Omega_{p}^{T}\right]^{T}=0$, where $D(q)$ is an $(N-n) \times N$ matrix. This is a system of equations that gives necessary and sufficient conditions for the feasibility of $\left[\Omega_{a}^{T}, \Omega_{p}^{T}\right]^{T}$. By combining $A(q)$ and $D(q)$, a $N \times(N+n)$ matrix $L(q)$ is introduced and fully defines the instantaneous kinematics of the mechanism by the velocity equation $L(q)\left[T^{T}, \Omega_{a}^{T}, \Omega_{p}^{T}\right]^{T}=0$. According to this formulation, Zlatanov et al. [2] define six types of singular configurations: i) redundant input: there exist $\Omega_{a} \neq 0$ and $\Omega_{p} \neq 0$ to satisfy the velocity equation while $T=0$, ii) redundant output: there exist $T \neq 0$ and $\Omega_{p} \neq 0$ to satisfy the velocity equation while $\Omega_{a}=0$, iii) impossible input: for some $\Omega_{a} \neq 0$ the velocity equation cannot be satisfied for any combination of $T$ and $\Omega_{p}$, iv) impossible output: for some $T \neq 0$ the velocity equation cannot be satisfied for any combination of $\Omega_{a}$ and $\left.\Omega_{p}, \mathrm{v}\right)$ increased instantaneous mobility: if $\operatorname{rank} L<N$, then the instantaneous mobility is greater than the global mobility $\mu$ and both forward and inverse kinematic mappings are singular, and vi) redundant passive motion: there exists $\Omega_{p} \neq 0$ to satisfy the velocity equation while $\Omega_{a}=0$ and $T=0$. We note that this analysis does not explicitly define the configuration space of the PM and is simply instantaneous. It also continues to base the (instantaneous) kinematics in terms of the pre-selected inputs and outputs. These are limitations that we seek to remove in this thesis.

Although the framework proposed by Park and Kim [54] carries simi- 
larities to that of Gosselin and Angeles [1], they extend its framework by allowing for redundant actuation (more actuator dofs than output dofs) and by more explicitly recognising the role of differential geometry. Specifically, pseudo-Riemannian metrics are introduced on manifolds, namely the ambient space $K$, the joint configuration space $M$ of all joint variables in the absence of loop-closure constraints, and the end-effector space $N$. These metrics are expressed in local coordinates $\mathbf{x}, \mathbf{u}, \mathbf{f}$ on $K, M, N$ respectively by symmetric positive definite matrices $\mathbf{E}, \mathbf{G}$, and $\mathbf{H}$. Assuming a well-defined (local) forward kinematics $\mathbf{f}=\mathbf{f}(\mathbf{u})$, there is a Jacobian $J=D_{\mathbf{u}} \mathbf{f}$, and an embedding of the configuration space given in local coordinates by $\mathbf{x}=\mathbf{x}(\mathbf{u})$. Then $\mathbf{G}=\left(D_{\mathbf{u}} \mathbf{x}\right)^{T} \mathbf{E}\left(D_{\mathbf{u}} \mathbf{x}\right)$, where $\mathbf{E}$ may be semi-definite, depending on the choice of actuators. Based on this approach three possible singularity types are identified: i) a configuration space singularity ( $M$ is defined by $\mathbf{\Phi}(\mathbf{x})=0$ and $D_{\mathbf{x}} \mathbf{\Phi}$ is singular), ii) an actuator singularity: the rank of $\mathbf{G}$ decreases, and iii) an end-effector singularity: the rank of $\mathbf{J}$ decreases. Although the technical setting is differential geometry on manifolds, explicit computations are largely lacking and as with the earlier research, assumptions are made about the role of actuated joints and end-effector or output variables.

The complexity of PM kinematics and singularity analysis continued to pose a challenge, but by the beginning of this decade (2010s) the central role of the configuration space had become apparent. While there was recognition of the global geometric approach, instantaneous kinematics remained the main tool. For example, Müller [52] constructed an approach which is focused on an instantaneous kinematics model and analyses a PM in a given configuration. This approach begins with considering a vector of joint variables $\mathbf{q} \in \mathbb{V}^{n}$, where $\mathbb{V}^{n}$ is the joint space of a PM. If $\mathbf{h}$ is the set of loop constraints (i.e. geometric constraints), then solutions for $\mathbf{h}(\mathbf{q})=0$ determine the configuration space $V$ that is the set of all feasible configurations. The output mapping $f_{O}: V \rightarrow S E(3)$ determines the position and orienta- 
tion of the end-effector in terms of a given $\mathbf{q} \in V$. The workspace $\mathcal{W}$ of the end-effector is then defined as the set of all feasible positions and orientations of the end-effector so that $\mathcal{W} \subset S E(3)$. In addition, the relation between active inputs and motions of the PM is described via the input mapping $f_{I}: V \rightarrow \mathcal{I}$, where $\mathcal{I}$ is the input space. In particular, the relation between joint inputs and configurations is reversed from earlier models. Hence, $f_{I}$ assigns feasible inputs to any configuration of PM. According to this formulation, $f_{I}$ and $f_{O}$ are considered as mappings from the configuration space $V$ to $\mathcal{I}$ and $\mathcal{W}$, respectively. Associated with the $\mathbf{h}, f_{O}$, and $f_{I}$, there are Jacobian matrices $\mathbf{J}, \mathbf{J}_{O}$, and $\mathbf{J}_{I}=\left[\mathbf{J}_{a}, \mathbf{J}_{p}\right]$, respectively. In this model, three types of singularities arise: i) configuration singularity: $\operatorname{rank} \mathbf{J}$ is not constant in any neighborhood of $\mathbf{q} \in V$, ii) input singularity: either rank $\mathbf{J}_{p}$ or $\operatorname{rank} \mathbf{J}_{a}$ is not constant in any neighborhood of $\mathbf{q} \in V$, and iii) output singularity: $\operatorname{rank} \mathbf{J}_{O}$ is not constant in any neighborhood of $\mathbf{q} \in V$. These singularities can occur simultaneously, and any possible combinations of them may lead to instantaneously impossible input motions, or instantaneously redundant inputs in the terminology of Zlatanov et al. [2].

At about the same time, a similar framework was proposed by Piipponen and Tuomela [61] based on algebraic geometry. They proposed a formulation to determine the constraints on configurations based on geometrical reasoning to provide polynomial equations for each joint without specifying which are to be actuated. They propose that only three basic constraint types are needed: i) coincidence constraint: given points in the coordinate systems of the rigid bodies are the same point in the coordinate system of the ambient space, ii) symmetric orthogonality constraint: two given vectors in the coordinate systems of the rigid bodies must be perpendicular to each other in the coordinate system of the ambient space, and iii) non-symmetric orthogonality constraint: given a vector and two points in the coordinate systems of the rigid bodies so that the difference of the points must be perpendicular to the 
vector in the coordinate system of the ambient space. By taking appropriate combinations of the basic types, many lower-pair joints may be defined. These constraints are identified as "local" (since they are defined on a single pair of jointed bodies). Following the same judgment as [2], these local constraints are distinguished from global constraints or closure-loop equations (which describe the structure of the mechanism as a whole). In fact, we show in this thesis that joint constraints are entirely sufficient to describe the configuration space since the loop-closure equations derive from them. The detailed analysis in Piipponen and Tuomela [61] is in terms of algebraic equations and geometry, whereas we use the setting of differential topology.

While it is not in the mainstream of robot/mechanism kinematics, it is worth noting that a constraint-based approach has been proposed previously. Kramer [62] was motivated by the challenge of symbolic or numerical computation for kinematic systems. He introduced the idea of a marker, which is made up of a point along with orthogonal $x-, z$-axes. Markers are attached to the rigid bodies and thus constraints between markers constrain the bodies. Joint constraints are then modelled as coincidence between a pair of markers. This approach is similar to that subsequently used by Piipponen and Tuomela [61]. While Kramer's goal was to replace algebra by geometry, his approach can be realised algebraically in terms of "absolute coordinates", in which the position and orientation of each rigid link is identified in terms of a reference frame. This is the approach we will adopt in this thesis.

Finally, we note that recent work by Bohigas et al. [53] also adapts the earlier work of Zlatanov et al. [2] to accommodate the perspective of Müller [52] by treating the input and output mappings as functions from the configuration space. Therefore, the framework we present in this thesis is in line with other current research. However, it combines features of these in a novel way that appears more comprehensive than any earlier work and which is demonstrated to give geometric insight into the kinematics and singularity 
analysis of many types of manipulator.

\subsection{A constraint-based approach}

In this thesis, a comprehensive approach to multi-body mechanism kinematics is introduced enabling analysis of singularities of both serial and parallel mechanisms. Existing approaches, like those of Gosselin and Angeles [1], Zlatanov et al. [2] and Park and Kim [3], make use of a combination of joint freedoms and constraints. However, a uniquely constraint-based approach seems to be more desirable and universal. This is not entirely new - a few other approaches, like those of Kramer [62] and Piipponen and Tuomela [61] concentrate on constraints. The former describes configurations of a set of rigid bodies using a geometric-based formulation for the purpose of defining a symbolic algorithmic approach to kinematics, while the latter is an algebra-based method suitable for Gröbner basis techniques from algebraic geometry. Similarly, Bohigas et al. [53] have recently proposed a model of this sort. However, all these approaches either implicitly or explicitly retain the use of joint variables as an intrinsic element of the kinematic model.

We also bear in mind that design parameters play an important role in singularities occurring. By taking into account variation of design parameters for a family of manipulators, we capture how the topological properties of its kinematics vary. A member of this family is identified by a specific choice of design parameters which may or may not have singularities.

The advantage of this consideration is that if we are asked to design a manipulator with a given set of design parameters, it will help us to know for what choices of design parameters the manipulator may have singularities. In principle, we may also be able to identify overconstrained manipulators in a family, where there is finite mobility that would not be expected. For such manipulators, in some sense, all configurations are singular. However, we have not been able to pursue this line of research. 
The proposed representation removes direct references to the freedoms and focuses on the kinematic constraint mapping (KCM) based on constraints imposed by joints on the relative motion of links, rather than the freedom of motion they permit. The mapping is expressed in terms of parameters called pose variables, which are displacement coordinates of links with respect to a reference frame. The properties of a given mechanism can be described by means of this representation, locally and globally. Also, the KCM will tell us how the choice of design parameters affects these properties within a family of mechanisms.

The KCM determines a subset in the space of pose variables called the configuration space (C-space). Its Jacobian (the matrix of first-order partial derivatives) may drop rank for some values of the pose variables, so that the C-space has singularities. By parametrising the C-space in terms of design parameters, we seek those choices of design parameters for which the Cspace has singularities, which we call Grashof-type conditions for the family of manipulators.

As the second step towards full understanding of singularities, we develop the theory to incorporate the actuator space (A-space) and workspace (W-space), based on choice of actuated joints (inputs) and on the manipulator's workspace or end-effector (outputs). This provides us with a framework for analysing singularities for forward and inverse kinematics via input and output mappings defined on the manipulator's C-space.

To summarise, this approach has a number of advantages over many previous formulations:

(i) The roles of the variables describing link positions-pose variables in our terminology — and joint variables are clearly distinguished. This distinction has been blurred because often joint variables act as surrogates for a pose variables, or vice versa.

(ii) The analysis of singularities is global - the definition of the C-space 
captures all configurations of all the links in the manipulator in terms of its pose variables, so singular configurations are explicitly identified. Rather than treating Jacobians as purely local realisations of the instantaneous kinematic relationship between joint velocities and link velocities, they are matrices of partial derivatives of globally defined mappings.

(iii) The KCM approach works for serial and parallel manipulators. It does not require inclusion of "loop-closure" constraints, which are simply corollaries of the joint constraints. As a result, it will be easy to see why, for example, the forward kinematics of a serial mechanism are well defined.

\subsection{Thesis structure}

We start with some mathematical basics including background from differential geometry, Lie theory, and associative algebra. In Chapter 2, we review the definition of a Lie group and discuss Euclidean groups, along with corresponding methods of parametrisation in more detail. An element of the Euclidean group is used to describe rigid displacement. We review dual quaternions as an efficient tool to express a rigid displacement. In the last section of this chapter, we introduce the idea of pose space which consists of pose variables of all moving components of a manipulator.

Chapter 3 is allocated to the Lie algebra of Euclidean groups $S O(n)$ and $S E(n), n=2,3$. We will start with several representations of Lie algebras. The relation between the Euclidean Lie algebra and screw theory will be discussed in detail. Following this, the Mozzi-Chasles theorem [63, 64], the connections between line geometry and Plücker coordinates and screws - a description of finite and instantaneous rigid body displacement - is given. Next, the exponential function will be introduced as a mapping which transforms an element of the Lie algebra to an element of the Lie group and which gives rise to 1-parameter subgroups. The last section will be allocated to infinitesimal displacement in the pose space. 
In Chapter 4, we introduce the core idea of our approach. Here, we model each type of kinematic pair (joint) with a geometric object associated to each joined link. Identification of these objects provides us with a set of constraint equations for the joint. For a given manipulator with a number of different joints, the collection of all constraint equations is a vector-valued function called the kinematic constraint mapping (KCM). The $\mathrm{KCM}$ determines a manifold called the configuration space (C-space) whose points correspond to feasible configurations for a given manipulator. First-order partial derivatives of the KCM with respect to pose variables form a Jacobian matrix which is used for the purpose of singularity analysis. In the last section, we express a joint in terms of its relative freedom, corresponding to a joint variable, which can be described in terms of pose variables of two adjacent components connected at the joint.

Chapter 5 describes a comprehensive singularity analysis of a given manipulator. The first part will discuss the most fundamental type of singularities, C-space singularities. By considering transversality for the KCM, we indicate how C-space singularities may be characterised by a Grashof-type condition only defined in terms of design parameters. In the second part of the chapter, based on the choice of inputs and outputs, an actuator space (A-space) and workspace (W-space) will be determined. The resulting input map and output map are defined and corresponding singularities described.

Finally in Chapters 6-9, we will apply our constraint-based approach to several serial and parallel manipulators and perform a full kinematic analysis including singularities. Some of the results in the thesis have been published already or are planned to appear [65, 66, 67, 68, 69]. 


\section{Chapter 2}

\section{Euclidean group}

In this chapter we study representation of the displacement of a rigid object in Euclidean space. Such displacements are expressed by means of the Euclidean group which is a Lie group. A Lie group is a group with the additional structure of a differentiable manifold, such that its operations are differentiable.

\section{$2.1 \quad$ Lie group}

We begin with the definition of a group [70, 71]

Definition 2.1. A group $G$ is a set with a finite or infinite number elements on which a binary operation $\left(g_{1}, g_{2}\right) \mapsto g_{1} g_{2}$ is defined satisfying the following four properties

- closure: $\forall g_{1}, g_{2} \in G, g_{1} g_{2} \in G$

- associativity: $\forall g_{1}, g_{2}, g_{3} \in G, \quad g_{1}\left(g_{2} g_{3}\right)=\left(g_{1} g_{2}\right) g_{3}$

- identity: $\exists e \in G, \forall g \in G \quad g e=e g=g$

- inversion: $\exists g^{-1} \in G, \forall g \in G \quad g g^{-1}=g^{-1} g=e$ 
Note that $\forall g_{1}, g_{2} \in G,\left(g_{1} g_{2}\right)^{-1}=g_{2}^{-1} g_{1}^{-1}$. A non-empty subset of a group that is closed under the binary operation and inversion is called a subgroup. Assume $H$ is a subgroup of $G$ and $g \in G$, then $g H:=\{g h: h \in H\}$ is a left coset of $H$ whilst $H g:=\{h g: h \in H\}$ is a right coset of $H$. It can immediately be concluded that $\forall g_{1}, g_{2} \in G$

$$
g_{1} H=g_{2} H \Longleftrightarrow g_{1}^{-1} g_{2} \in H, \quad H g_{1}=H g_{2} \Longleftrightarrow g_{1} g_{2}^{-1} \in H
$$

Consequently, it can be deduced that left cosets (similarly right cosets) partition $G$ because these conditions define an equivalence relation on $G$ and the cosets equivalence classes.

Generally speaking, $g H$ and $H g$ are distinct subsets of $G$ although they both contain $g$.

Definition 2.2. For all $g \in G$, if the left and right cosets coincide $g H=H g$, then $H$ is a normal subgroup of $G$

$$
H \triangleleft G:=\left\{g H g^{-1}: g \in G\right\}
$$

Let us consider two groups along with their binary operations $\left(G_{1}, \cdot\right)$ and $\left(G_{2}, \times\right)$.

Definition 2.3. A function $\phi: G_{1} \rightarrow G_{2}$ is a group homomorphism if it preserves the binary operation

$$
\forall g_{1}, g_{2} \in G_{1}, \phi\left(g_{1}\right) \cdot \phi\left(g_{2}\right)=\phi\left(g_{1} \times g_{2}\right) \in G_{2}
$$

Based on this definition, it can be concluded that a group homomorphism must also preserve the identity and group inversion; that is

- $\phi\left(g_{1}\right)=\phi\left(e_{1} g_{1}\right)=\phi\left(e_{1}\right) \cdot \phi\left(g_{1}\right) \Rightarrow \phi\left(e_{1}\right)=e_{2}$ 
- $\phi(g) \cdot \phi\left(g^{-1}\right)=\phi\left(g g^{-1}\right)=\phi\left(e_{G_{1}}\right)=e_{G_{2}} \therefore \phi(g)^{-1}=\phi\left(g^{-1}\right)$

The kernel of a homomorphism $\phi$ is $\operatorname{ker} \phi=\left\{g_{1} \in G_{1} \mid \phi\left(g_{1}\right)=e_{G_{2}}\right\}$ where $e_{G_{2}}$ is the identity element of $G_{2}$; $\operatorname{ker} \phi$ is a normal subgroup of $G_{1}$. A homomorphism $\phi$ is called an isomorphism if it is a bijection (i.e. one-to-one and onto). In particular, it has an inverse which is also a homomorphism. In this case, the two groups are isomorphic.

Now, we can introduce the idea of a Lie group. This requires some differential topology and in particular the definition of a differentiable manifold. This is quite technical and we refer to, for example, [72, 73]. An alternative simpler but more restrictive approach is in [74]. For our purpose, it will sufficient to say that a differentiable manifold is a subset $M$ of some Euclidean space $\mathbb{R}^{p}$ such that a neighbourhood in $M$ of every point $\mathbf{x} \in M$ can be mapped bijectively to an open subset of some $\mathbb{R}^{m}(m \leq p$ fixed) and that this mapping or $\operatorname{chart} \varphi$ is differentiable and has differentiable inverse.

In brief, $M$ is locally Euclidean whose dimension is $m$. A simple example is the unit sphere

$$
S^{2}:=\left\{\mathbf{x} \in \mathbb{R}^{3} \mid\|\mathbf{x}\|=1\right\}
$$

Open hemispheres, $x_{i}>0$ or $x_{i}<0 ; i=1,2,3$ can be mapped bijectively to the open unit disc in $\mathbb{R}^{2}$ by omitting the $i^{\text {th }}$ coordinate. A typical inverse has the form

$$
\left(y_{1}, y_{2}\right) \mapsto\left(\sqrt{1-y_{1}^{2}-y_{2}^{2}}, y_{1}, y_{2}\right)
$$

which is differentiable since $y_{1}^{2}+y_{2}^{2}<1$. The unit sphere $S^{2}$ is a 2 -dimensional (differentiable) manifold.

If $M, N$ are differentiable manifolds and $f: M \rightarrow N$ a function, then $f$ is differentiable at $\mathbf{x} \in M$ if there is a chart $\varphi$ on $M$ at $\mathbf{x}$ and a chart $\psi$ on $N$ at $f(\mathbf{x})$ such that $\psi \circ f \circ \varphi^{-1}$ is differentiable at $\varphi(\mathbf{x})$. This composition or local representative maps between $\mathbb{R}^{m}$ and $\mathbb{R}^{n}$ where $m=\operatorname{dim} M$ and $n=\operatorname{dim} N$.

Definition 2.4. A Lie group is a group which is also a differentiable manifold 
and whose binary and inverse operations are differentiable.

Note that the binary operation is a map $G \times G \rightarrow G$. If $G$ is a manifold then so is the Cartesian product $G \times G$.

\section{$2.2 \quad$ Euclidean groups}

In the previous section, we briefly reviewed the definition of a Lie group. The Euclidean group is central to robot kinematics and is a Lie group that expresses the symmetry of Euclidean space [75].

Definition 2.5. Euclidean space is the space of $n$-vectors of real numbers $\mathbf{x}:=\left(x_{1}, x_{2}, \ldots, x_{n}\right) \in \mathbb{R}^{n}$ together with the Euclidean inner product

$$
\forall \mathbf{x}, \mathbf{y} \in \mathbb{R}^{n} \quad\langle\mathbf{x}, \mathbf{y}\rangle=\mathbf{x}^{T} \mathbf{y}=\sum_{i=1}^{n} x_{i} y_{i}
$$

where $T$ as a superscript stands for transpose. Definition 2.5 gives rise to the Euclidean norm $\|\mathbf{x}\|_{2}=\sqrt{\langle\mathbf{x}, \mathbf{x}\rangle}$, which defines the Euclidean length of $\mathbf{x}$, and hence the Euclidean metric (or distance function) $d: \mathbb{R}^{n} \times \mathbb{R}^{n} \rightarrow \mathbb{R}$

$$
d(\mathbf{x}, \mathbf{y})=\|\mathbf{x}-\mathbf{y}\|_{2}
$$

This distance function gives rise to an isometry that preserves the Euclidean metric.

We are interested in functions that preserve the inner product and the metric. First, let us consider linear transformations $f_{r}: \mathbb{R}^{n} \rightarrow \mathbb{R}^{n}$ that preserve the Euclidean inner product i.e. $\forall \mathbf{x}, \mathbf{y} \in \mathbb{R}^{n},\left\langle f_{r}(\mathbf{x}), f_{r}(\mathbf{y})\right\rangle=\langle\mathbf{x}, \mathbf{y}\rangle$ are called orthogonal and $O(n)$ denotes the set of these transformations.

Lemma 2.6. $A \in O(n)$ if and only if $A^{T} A=I_{n}{ }^{1}$.

\footnotetext{
${ }^{1} I_{n}$ stands for the $n$-dimensional identity matrix.
} 
A proof can be found in standard texts, for example [76]. It follows that $O(n)$ is a group since products and inverses will also satisfy the equation.

The orthogonal group $O(n)$ also forms a manifold because Lemma 2.6 implies that $O(n):=g_{s}^{-1}\left(I_{n}\right)$ where $g_{s}(A)=A^{T} A$. Since $g_{s}(A)$ is always symmetric, it can be shown that $g_{s}$ is regular at any $A \in O(n)$ that means $O(n)$ is a manifold of dimension $\frac{1}{2} n(n-1)$ (see e.g. [31]). Furthermore, the operations of matrix multiplication and inverse are differentiable. The latter can be seen based on the matrix inverse formula

$$
A^{-1}=\frac{\operatorname{adj} A}{\operatorname{det} A}
$$

where $\operatorname{adj} A$ and $\operatorname{det} A$ stand for the adjugate $^{2}$ and the determinant of $A$, respectively. Therefore, entries in $A^{-1}$ are ratios of polynomials in the entries of $A$ and the denominator is non-zero. Hence $O(n)$ is a Lie group.

Since $\operatorname{det} A=\operatorname{det} A^{T}$ and $\operatorname{det} I_{n}=1$, so $\operatorname{det} A^{T} A=\operatorname{det} A^{T} \operatorname{det} A=$ $\operatorname{det} A^{2}=1$, which implies that $\operatorname{det} A= \pm 1$. As a result, the manifold associated to $O(n)$ is made up of two disjoint subsets. Matrices such that $\operatorname{det} A=-1$ represent reflections and reverse orientation in $\mathbb{R}^{n}$ whilst matrices with $\operatorname{det} A=+1$ preserve orientation. The latter form a Lie group called the special orthogonal group $S O(n)$ that is a connected subgroup and is a Lie group with the same dimension as $O(n)$. Accordingly, elements of $S O(n)$ are rotations about the origin $\mathbf{o} \in \mathbb{R}^{n}$. Although reflections are proper orthogonal transformations, they are irrelevant in the kinematics of mechanisms because no mechanical component can produce a reflection. Hence from now on, we will only focus on $S O(n)$ as the group of rotations. Note that rotations, in general, form a non-abelian group; that is, they do not commute i.e. for $A_{1}, A_{2} \in S O(n) A_{1} A_{2} \neq A_{2} A_{1}$. The case $n=2$ is the exception.

Definition 2.7. A Euclidean transformation is an isometry $f: \mathbb{R}^{n} \rightarrow \mathbb{R}^{n}$;

\footnotetext{
${ }^{2}$ The adjugate of a square matrix is the transpose of its cofactor matrix. It is also known as the adjoint and the adjunct.
} 
that is

$$
\forall \mathbf{x}, \mathbf{y} \in \mathbb{R}^{n}, \quad d(\mathbf{X}, \mathbf{Y})=d(\mathbf{x}, \mathbf{y})
$$

where $\mathbf{X}=f(\mathbf{x})$ and $\mathbf{Y}=f(\mathbf{y})$.

Euclidean transformations are bijective. If we assume $f(\mathbf{x})=f(\mathbf{y})$, then $d(f(\mathbf{x}), f(\mathbf{y}))=0$. Hence, $d(\mathbf{x}, \mathbf{y})=0$ and so $\mathbf{x}=\mathbf{y}$ and $f$ is injective (oneto-one). Surjectivity (onto) is harder to prove but follows from (2.5). Clearly, the composition of two Euclidean transformations is also in the Euclidean space. Note that if $f$ is a Euclidean transformation, there is an inverse function associated to it, $f^{-1}: \mathbb{R}^{n} \rightarrow \mathbb{R}^{n}$. If $\mathbf{x}=f^{-1}(\mathbf{X})$ and $\mathbf{y}=f^{-1}(\mathbf{Y})$, then since $f$ is an isometry, so $d\left(f^{-1}(\mathbf{X}), f^{-1}(\mathbf{Y})\right)=d(\mathbf{x}, \mathbf{y})=d(f(\mathbf{x}), f(\mathbf{y}))=$ $d(\mathbf{X}, \mathbf{Y})$, showing that $f^{-1}$ is also an isometry. Therefore, they form a group, called the Euclidean group, $E(n)$. Since orthogonal transformations preserve the Euclidean norm, they are also Euclidean transformations which fix the origin $\mathbf{o} \in \mathbb{R}^{n}$.

A translation is linear transformation $f_{t}: \mathbb{R}^{n} \rightarrow \mathbb{R}^{n}, \mathbf{X}=f_{t}(\mathbf{x})=\mathbf{x}+\mathbf{t}$ that shifts every point $\mathbf{x} \in \mathbb{R}^{n}$ by the same distance and in the same direction. Clearly, every translation is an isometry of $\mathbb{R}^{n}$ as the Euclidean metric is preserved. Let us assume $\mathbf{x}, \mathbf{y} \in \mathbb{R}^{n}$ that are transformed by $\mathbf{t} \in \mathbb{R}^{n}$ so $\mathbf{X}=\mathbf{x}+\mathbf{t}$ and $\mathbf{Y}=\mathbf{y}+\mathbf{t}$ then $d(\mathbf{X}, \mathbf{Y})=\mathbf{x}+\mathbf{t}-\mathbf{y}-\mathbf{t}=\mathbf{x}-\mathbf{y}=d(\mathbf{x}, \mathbf{y})$. The set of all translations establishes the translation group that is a Lie group isomorphic to $\mathbb{R}^{n}$. Translations form an abelian group; that is, they are commutative as for $\mathbf{t}_{1}, \mathbf{t}_{2} \in \mathbb{R}^{n}, \mathbf{t}_{1}+\mathbf{t}_{2}=\mathbf{t}_{2}+\mathbf{t}_{1}$.

The following theorem confirms that these two types of isometry - orthogonal and translation - generate all Euclidean transformations (for proof see e.g. [77] Page 26).

Theorem 2.8. Every Euclidean transformation $f: \mathbb{R}^{n} \rightarrow \mathbb{R}^{n}$ can be uniquely written as a composition of an orthogonal transformation followed by a translation. 
Based on Theorem 2.8, it follows that an element of $E(n)$ can be represented uniquely by $g:=(A, \mathbf{t})$ where $A \in O(n)$ and $\mathbf{t} \in \mathbb{R}^{n}$. The corresponding transformation is defined by

$$
\mathbf{X}=g \mathbf{x}=(A, \mathbf{t}) \mathbf{x}=A \mathbf{x}+\mathbf{t}
$$

The special Euclidean group $S E(n)$ is the connected subgroup in $E(n)$ represented by pairs $(A, \mathbf{t})$, where $A \in S O(n)$. Thus $S E(n)$ is the orientationpreserving Euclidean transformations on which we will concentrate from now on.

Given $g_{1}, g_{2} \in S E(n) ; g_{1}:=\left(A_{1}, \mathbf{t}_{1}\right), g_{2}:=\left(A_{2}, \mathbf{t}_{2}\right)$, then $g_{1}$ acts on $\mathbf{x} \in \mathbb{R}^{n}$ so $\mathbf{X}_{1}=A_{1} \mathbf{x}+\mathbf{t}_{1}$ and $g_{2}$ acts on $\mathbf{X}_{1}$ so $\mathbf{X}_{2}=A_{2} \mathbf{X}_{1}+\mathbf{t}_{2}$. The resultant of the composition of transformations, $g_{2} g_{1}$, is $\mathbf{X}_{2}=A_{2} A_{1} \mathbf{x}+\left(A_{2} \mathbf{t}_{1}+\mathbf{t}_{2}\right)$. Thus, the group operation is defined by

$$
\left(A_{2}, \mathbf{t}_{2}\right)\left(A_{1}, \mathbf{t}_{1}\right)=\left(A_{2} A_{1}, A_{2} \mathbf{t}_{1}+\mathbf{t}_{2}\right)
$$

Definition 2.9. Given a group $G$ acting on a vector space $V$, the semi-direct product is the group $G \ltimes V$ with product $\left(g_{2}, \mathbf{v}_{2}\right) \cdot\left(g_{1}, \mathbf{v}_{1}\right)=\left(g_{2} g_{1}, g_{2} \mathbf{v}_{1}+\mathbf{v}_{2}\right)$ where $g_{1}, g_{2} \in G$ while $\mathbf{v}_{1}, \mathbf{v}_{2} \in V$.

Theorem 2.8 and equation (2.7) give that $S E(n)$ is isomorphic to the semi-direct product of the rotations $S O(n)$ with the translations $\mathbb{R}^{n}$

$$
S E(n) \cong S O(n) \ltimes \mathbb{R}^{n}
$$

Note that the action of the rotation $A_{2}$ on the translation $\mathbf{t}_{1}$ stops this being a direct product. It follows that $S E(n)$ is a Lie group whose dimension is

$$
p=\operatorname{dim} S O(n)+\operatorname{dim} \mathbb{R}^{n}=\frac{1}{2} n(n-1)+n=\frac{1}{2} n(n+1)
$$

It is clear that $g_{t}:=\left(I_{n}, \mathbf{t}\right)$ represents a translation in $\mathbb{R}^{n}$ whereas $g_{r}:=(A, \mathbf{0})$ 
describes a rotation about the origin $\mathbf{o} \in \mathbb{R}^{n}$. The translation subgroup $I_{n} \ltimes \mathbb{R}^{n}$ is a normal subgroup of $S E(n)$ whereas $S O(n) \ltimes\{\mathbf{0}\}$ is not normal.

It is common to represent an element of $S E(n)$ using the homogeneous representation as an $(n+1 \times n+1)$ matrix [46, 70]

$$
g:={ }_{1}^{n}\left[\begin{array}{cc}
n & 1 \\
A & \mathbf{t} \\
\mathbf{0}^{T} & 1
\end{array}\right]
$$

where $\mathbf{0}^{T}$ is a row vector, which will be only written as $\mathbf{0}$ from now on. In the homogeneous representation, the composition of two Euclidean transformations $g_{1}, g_{2}$, can be expressed by matrix multiplication

$$
\left[\begin{array}{cc}
A_{2} & \mathbf{t}_{2} \\
\mathbf{0} & 1
\end{array}\right]\left[\begin{array}{cc}
A_{1} & \mathbf{t}_{1} \\
\mathbf{0} & 1
\end{array}\right]=\left[\begin{array}{cc}
A_{2} A_{1} & A_{2} \mathbf{t}_{1}+\mathbf{t}_{2} \\
\mathbf{0} & 1
\end{array}\right]
$$

Similarly, the inverse operation on an element of $S E(n)$ is given by the matrix inverse

$$
g^{-1}:=\left[\begin{array}{cc}
A & \mathbf{t} \\
\mathbf{0} & 1
\end{array}\right]^{-1}=\left[\begin{array}{cc}
A^{T} & -A^{T} \mathbf{t} \\
\mathbf{0} & 1
\end{array}\right]
$$

thus $g^{-1}=\left(A^{T},-A^{T} \mathbf{t}\right)$. This provides a group representation of $S E(n)$ on $\mathbb{R}^{n+1}$.

Note that the action of $S E(n)$ on $\mathbf{x} \in \mathbb{R}^{n}$, as in (2.5), is given in the homogeneous representation for given coordinates by setting $x_{n+1}=1$ so that if $\mathbf{x} \in \mathbb{R}^{n}$

$$
\left[\begin{array}{c}
\mathbf{X} \\
1
\end{array}\right]=\left[\begin{array}{ll}
A & \mathbf{t} \\
0 & 1
\end{array}\right]\left[\begin{array}{l}
\mathbf{x} \\
1
\end{array}\right]
$$

In the next section, we will show how to assign a Euclidean coordinate system to a rigid body and in a mechanism with a number of bodies how their coordinates interact. A coordinate system is parametrised by a set of parameters which will be employed as a representation of the Euclidean 
group.

\subsection{Coordinate frames and pose variables}

In the kinematics of a rigid body motion, we can make use of the Euclidean group by assigning a Euclidean coordinate system to a rigid body that is free to move in ambient space, along with its own coordinate system. It is an underlying assumption that the components of a robot mechanism are rigid and that the space in which they move has an inherently Euclidean structure. Nevertheless, there is not usually a single natural choice of coordinates so we are free to assign a coordinate system in a way that suits our analysis.

A Euclidean coordinate system in an $n$-dimensional space consists of a choice of a point as the origin $\mathbf{o} \in \mathbb{R}^{n}$ and $n$ direction vectors $\mathbf{e}_{1}, \ldots, \mathbf{e}_{n}$ positioned at $\mathbf{o}$ that are of unit length and mutually orthogonal, thus they form an orthonormal basis. Consequently, every point in the Euclidean space $\mathbf{x} \in \mathbb{R}^{n}$ is identified as a vector sum

$$
\mathbf{x}=x_{1} \mathbf{e}_{1}+\ldots+x_{n} \mathbf{e}_{n}
$$

and hence it is uniquely represented by its coordinates $\mathbf{x}:=\left(x_{1}, \ldots, x_{n}\right)$.

A coordinate system attached to a moving body is called a moving frame $M$. The body moves in an ambient space equipped with a coordinate system is called the reference frame $R$. Now, the question is how are a moving frame and the reference frame related? Let us suppose a rigid body, with moving frame $M$, is located at a particular pose - position and orientation - in its ambient space with reference frame $R$. Since both frames are orthonormal, the transformation between coordinates $\mathbf{x} \in M$ and $\mathbf{X} \in R$ is Euclidean. Moreover, we will ensure that the coordinates are ordered so that the transformation is orientation-preserving. As a result, there is an element of $S E(n)$ defining the pose. We call a set of parameters for this transformation its pose 
variables. The transformation is sometimes called an absolute displacement of a moving frame $M$ in the ambient space (see, for instance, [78]).

We are only interested in Euclidean groups $S E(n)$ where $n=2,3$. They express 2-dimensional (plane) and 3-dimensional (space) kinematics, respectively. We will discuss these two groups in the following section. As we have already seen, different representations can be employed to describe an element of $S E(n)$. For $S E(3)$, for example, dual quaternions are helpful. In the following sections, we will discuss four common representations; namely Euler angles, Euler parameters, quaternions, and dual quaternions.

\subsection{Group parametrisations}

In this section, we will describe representations of a spatial rigid displacement. Planar displacements can be viewed as spatial displacements that preserve a plane in $\mathbb{R}^{3}$.

Spatial rigid displacements in 3-dimensional soace have six degrees of freedom which is the dimension of the Euclidean group $S E(3)$. They can be taken to be three rotations about the origin and three translation along the axes of the reference frame. There are several different parametrisations available. Some of them have singularities defined globally. Others are singularity-free everywhere but involve additional parameters and constraints.

Planar displacements form a 3-dimensional Lie group $S E(2)$ that we can view as a subgroup of $S E(3)$. Therefore, only three parameters are required in the planar case. We begin by presenting parametrisations of the rotations about the origin.

\subsubsection{Special orthogonal groups}

There are several parametrisations to describe a spatial rotation, which is an element of $S O(3)$. Euler angles represent rotations about three axes [79, 80]. 
These are usually about $z-, y$ - (or $x$-) and $z$-axes. However, any three axes (so long as adjacent ones are distinct) can be used. For convenience, we use the parametrisation $\left(\theta_{x}, \theta_{y}, \theta_{z}\right) \in \mathbb{R}^{3}$, where the $(3 \times 3)$ rotation matrix associated to each angle corresponds to rotation about $x$-, $y$ - and $z$-axes successively

$$
A_{x}=\left[\begin{array}{ccc}
1 & 0 & 0 \\
0 & c_{x} & -s_{x} \\
0 & s_{x} & c_{x}
\end{array}\right], A_{y}=\left[\begin{array}{ccc}
c_{y} & 0 & s_{y} \\
0 & 1 & 0 \\
-s_{y} & 0 & c_{y}
\end{array}\right], \quad A_{z}=\left[\begin{array}{ccc}
c_{z} & -s_{z} & 0 \\
s_{z} & c_{z} & 0 \\
0 & 0 & 1
\end{array}\right]
$$

where $c_{x}, s_{x}$ respectively stand for $\cos \theta_{x}$ and $\sin \theta_{x}$ and similarly for $c_{y}, s_{y}$ and $c_{z}, s_{z}$. Therefore, an arbitrary rotation about the origin in $\mathbb{R}^{3}$ can be expressed by multiplying those three rotation matrices which results in a $(3 \times 3)$ matrix

$$
A=A_{x} A_{y} A_{z}=\left[\begin{array}{ccc}
c_{y} c_{z} & -c_{y} s_{z} & s_{y} \\
c_{x} s_{z}+c_{z} s_{x} s_{y} & c_{x} c_{z}-s_{x} s_{y} s_{z} & -c_{y} s_{x} \\
s_{x} s_{z}-c_{x} c_{z} s_{y} & c_{z} s_{x}+c_{x} s_{y} s_{z} & c_{x} c_{y}
\end{array}\right]
$$

There are bounds on the choice of angle so that the parametrisation is not continuous on $S O(3)$. That is, the Euler angle coordinate involves a singularity that has similarities to, but is not the same thing as, gimbal lock, which is a mechanical/kinematic phenomenon (for more details see [81]).

Planar rotations belong to the Lie group $S O(2)$. Thought of as a subset of $S O(3)$, this rotation occurs about an axis normal to a chosen plane. If we think of that axis as the $z$-axis of the Cartesian coordinate system, then the only required Euler angle will be $\theta_{z} \in \mathbb{R}$. We can write an element of $S O(2)$ as the $(2 \times 2)$ submatrix

$$
\left[\begin{array}{cc}
c_{z} & -s_{z} \\
s_{z} & c_{z}
\end{array}\right]
$$

The underlying manifold of $S O(2)$ is therefore the unit circle $S^{1} \subseteq \mathbb{R}^{2}$. In this case, $\theta_{z} \in \mathbb{R}$ can be used as a continuous parameter without singularities. 


\subsubsection{Quaternions and spatial rotations}

We introduce quaternions ${ }^{3}$ and show how unit quaternions can be employed to represent spatial rotations. The quaternions form a 4-dimensional space over the real numbers which we denote by $\mathbb{H}$. They form an associative algebra by introducing a multiplication operation. Consider $\{1, \mathrm{i}, \mathrm{j}, \mathrm{k}\}$ as a basis, the following relations between these basis elements

$$
\begin{array}{lll}
\mathrm{i} 1=1 \mathrm{i}=\mathrm{i}, & \mathrm{j} 1=1 \mathrm{j}=\mathrm{j}, & \mathrm{k} 1=1 \mathrm{k}=\mathrm{k}, \\
\mathrm{ij}=\mathrm{k}, & \mathrm{jk}=\mathrm{i}, & \mathrm{ki}=\mathrm{j}, \\
\mathrm{j} \mathrm{i}=-\mathrm{k}, & \mathrm{kj}=-\mathrm{i}, & \mathrm{ik}=-\mathrm{j}, \\
\mathrm{i}^{2}=\mathrm{j}^{2}=\mathrm{k}^{2}=\mathrm{ijk}=-1 &
\end{array}
$$

determine a non-commutative multiplication on $\mathbb{H}$.

Therefore, a quaternion is written in the form $\mathbf{q}=e_{0}+e_{1} \mathrm{i}+e_{2} \mathrm{j}+e_{3} \mathrm{k}$ where $e_{i} \in \mathbb{R}, i=0,1,2,3$. It is convenient to write this in the form $\mathbf{q}:=$ $\left(e_{0}, \mathbf{e}\right)$ where $\mathbf{e}=e_{1} \mathrm{i}+e_{2} \mathrm{j}+e_{3} \mathrm{k}$ can be identified with a 3 -vector in $\mathbb{R}^{3}$. We call $e_{0}$ the scalar or real part of $\mathbf{q}$ while $\mathbf{e}$ is called a pure (imaginary) quaternion. We often identify a scalar quaternion $\left(e_{0}, \mathbf{0}\right)$ with $e_{0}$ and a pure quaternion $(0, \mathbf{e})$ with $\mathbf{e}$. One can immediately observe that a vector $\mathbf{v} \in \mathbb{R}^{3}$ can be represented by a pure quaternion $\mathbf{q}:=(0, \mathbf{v})$.

Given two quaternions $\mathbf{q}_{1}=\left(e_{0}, \mathbf{e}\right)$ and $\mathbf{q}_{2}=\left(f_{0}, \mathbf{f}\right)$, addition is represented componentwise

$$
\mathbf{q}_{1}+\mathbf{q}_{2}=\left(e_{0}+f_{0}, \mathbf{e}+\mathbf{f}\right)
$$

while multiplication (quaternion product) can be written in the form

$$
\mathbf{q}_{1} \mathbf{q}_{2}=\left(\left(e_{0} f_{0}-\mathbf{e} \cdot \mathbf{f}\right),\left(e_{0} \mathbf{f}+f_{0} \mathbf{e}+\mathbf{e} \times \mathbf{f}\right)\right)
$$

\footnotetext{
${ }^{3}$ Quaternions were first introduced by W. R. Hamilton in 1843 [7, 82].
} 
where $\mathbf{e} \cdot \mathbf{f}$ and $\mathbf{e} \times \mathbf{f}$ are dot product and cross product of vectors in $\mathbb{R}^{3}$.

A quaternion $\mathbf{q}:=\left(e_{0}, \mathbf{e}\right) \in \mathbb{H}$ has a conjugate of the form

$$
\mathbf{q}^{*}:=\left(e_{0},-\mathbf{e}\right) \in \mathbb{H}
$$

The product of a quaternion with its conjugate gives a non-negative real number

$$
\mathbf{q q}^{*}=\mathbf{q}^{*} \mathbf{q}=e_{0}^{2}+e_{1}^{2}+e_{2}^{2}+e_{3}^{2}
$$

We call its square root the norm of $\mathbf{q}$, denoted $\|\mathbf{q}\|$. The inverse of a non-zero quaternion is then given by

$$
\mathbf{q}^{-1}=\frac{\mathbf{q}^{*}}{\|\mathbf{q}\|^{2}}
$$

A unit quaternion $\mathbf{q} \in \mathbb{H}$ is one that satisfies $\|\mathbf{q}\|=1$. The unit quaternions form a 3-sphere $S^{3} \subseteq \mathbb{R}^{4} \equiv \mathbb{H}$. Moreover, $S^{3}$ is a group with product and inverse given by quaternion product and conjugate and identity element $(1, \mathbf{0})$. Given $\mathbf{q}=\left(e_{0}, \mathbf{e}\right) \in S^{3}$, since $e_{0}^{2} \leq 1$ we can write $e_{0}=\cos (\theta)$ for some $0 \leq \theta \leq \pi$. Then, $\|\mathbf{e}\|=|\sin (\theta)|$ and we can write $\mathbf{q}=\cos \theta+\mathbf{u} \sin \theta$ where $\mathbf{u} \in \mathbb{R}^{3}$ and $\|\mathbf{u}\|=1$, that is, $\mathbf{u}$ is a pure unit quaternion.

Now consider the action of this unit quaternion $\mathbf{q} \in S^{3}$ on pure quaternions $\mathbf{v} \in \mathbb{R}^{3}$, through conjugation

$$
\mathbf{v} \mapsto \mathbf{q v q}^{*}
$$

This can be shown to have the following properties

- it is an isometry of $\mathbb{R}^{3}:\left\|\mathbf{q v q}^{*}\right\|=\|\mathbf{v}\|$

- it fixes $\mathbf{u}, \mathbf{q u q}^{*}=\mathbf{u}$

- it rotates the plane orthogonal to $\mathbf{u}$ through an angle $2 \theta$ 
It follows that $f_{\mathbf{q}}(\mathbf{v})=\mathbf{q v q}^{*}$ is an element of $S O(3)$. Since $f_{\mathbf{q}}=f_{-\mathbf{q}}$, there is a $2: 1$ homomorphism $S^{3} \rightarrow S O(3)$ given by $\mathbf{q} \mapsto f_{\mathbf{q}}$.

Viewed the other way round, a rotation (anti-clockwise) about an axis $\mathbf{u} \in \mathbb{R}^{3}$ by an angle $\theta$ can be represented by the unit quaternion $\cos (\theta / 2)+$ $\mathbf{u} \sin (\theta / 2)$. Composition of rotations is given by quaternion product and it is more straightforward to determine the axis and angle of rotation than using Euler angles. If $\mathbf{q}=e_{0}+e_{1} \mathrm{i}+e_{2} \mathbf{j}+e_{3} \mathrm{k}$ represents a rotation, then the condition $e_{0}^{2}+e_{1}^{2}+e_{2}^{2}+e_{3}^{2}=1$ is required. Furthermore, quaternions provide a smooth parametrisation of $S O(3)$, however, the representation is not unique since $-\mathbf{q}$ represents the same rotation.

It can be convenient to use the $(4 \times 4)$ matrix representation of $\mathbf{q}=$ $e_{0}+e_{1} \mathrm{i}+e_{2} \mathrm{j}+e_{3} \mathrm{k}$ as

$$
Q=\left[\begin{array}{cccc}
e_{0} & -e_{1} & -e_{2} & -e_{3} \\
e_{1} & e_{0} & -e_{3} & e_{2} \\
e_{2} & e_{3} & e_{0} & -e_{1} \\
e_{3} & -e_{2} & e_{1} & e_{0}
\end{array}\right]
$$

This is the linear representation of quaternions product by $\mathbf{q}$, that is if $\mathbf{q}, \mathbf{r} \in$ $\mathbb{H}$, then $\mathbf{q r}=Q \mathbf{r}$, where on the right hand side $\mathbf{r}$ is written as a 4-vector. Further, for $\mathbf{q} \in S^{3}, \mathbf{q}^{*}$ is represented by $Q^{T}$. Conjugation of the basis vectors $\mathrm{i}, \mathrm{j}, \mathrm{k}$ by $\mathbf{q}$ yields, for example

$$
\begin{aligned}
\mathbf{q} \mathbf{i} \mathbf{q}^{*} & =\left(e_{0}+e_{1} \mathrm{i}+e_{2} \mathrm{j}+e_{3} \mathrm{k}\right) \mathrm{i}\left(e_{0}-e_{1} \mathrm{i}-e_{2} \mathrm{j}-e_{3} \mathrm{k}\right) \\
& =0+\left(e_{0}^{2}+e_{1}^{2}-e_{2}^{2}-e_{3}^{2}\right) \mathrm{i}+2\left(e_{1} e_{2}+e_{0} e_{3}\right) \mathrm{j}+2\left(e_{1} e_{3}-e_{0} e_{2}\right) \mathrm{k}
\end{aligned}
$$

Equivalently, using matrix multiplication of matrices as in (2.20)

$$
Q \mathrm{I} Q^{T}=\left[\begin{array}{cccc}
e_{0} & -e_{1} & -e_{2} & -e_{3} \\
e_{1} & e_{0} & -e_{3} & e_{2} \\
e_{2} & e_{3} & e_{0} & -e_{1} \\
e_{3} & -e_{2} & e_{1} & e_{0}
\end{array}\right]\left[\begin{array}{cccc}
0 & -1 & 0 & 0 \\
1 & 0 & 0 & 0 \\
0 & 0 & 0 & -1 \\
0 & 0 & 1 & 0
\end{array}\right]\left[\begin{array}{cccc}
e_{0} & e_{1} & e_{2} & e_{3} \\
-e_{1} & e_{0} & e_{3} & -e_{2} \\
-e_{2} & -e_{3} & e_{0} & e_{1} \\
-e_{3} & e_{2} & -e_{1} & e_{0}
\end{array}\right]
$$




$$
=\left[\begin{array}{ccc}
0 & -e_{0}^{2}-e_{1}^{2}+e_{2}^{2}+e_{3}^{2} & \\
e_{0}^{2}+e_{1}^{2}-e_{2}^{2}-e_{3}^{2} & 0 & \\
2\left(e_{1} e_{2}+e_{0} e_{3}\right) & -2\left(e_{0} e_{2}-e_{1} e_{3}\right) & \\
2\left(e_{1} e_{3}-e_{0} e_{2}\right) & -2\left(e_{1} e_{2}+e_{0} e_{3}\right) & \\
-2\left(e_{1} e_{2}+e_{0} e_{3}\right) & -2\left(e_{1} e_{3}-e_{0} e_{2}\right) \\
2\left(e_{0} e_{2}-e_{1} e_{3}\right) & 2\left(e_{1} e_{2}+e_{0} e_{3}\right) \\
0 & -e_{0}^{2}-e_{1}^{2}+e_{2}^{2}+e_{3}^{2} \\
e_{0}^{2}+e_{1}^{2}-e_{2}^{2}-e_{3}^{2} & 0
\end{array}\right]
$$

and we can easily extract the vector form

$$
\left(0, e_{0}^{2}+e_{1}^{2}-e_{2}^{2}-e_{3}^{2}, 2\left(e_{1} e_{2}+e_{0} e_{3}\right), 2\left(e_{1} e_{3}-e_{0} e_{2}\right)\right)^{T}
$$

Similarly, we can obtain the results of conjugation by $\mathbf{q}$ on $\mathbf{j}$, k. We can write the results in the form of following matrix whose columns correspond to the pure quaternion parts of conjugation on the unit vectors $\mathrm{i}, \mathrm{j}, \mathrm{k}$

$$
\left[\begin{array}{ccc}
e_{0}^{2}+e_{1}^{2}-e_{2}^{2}-e_{3}^{2} & 2\left(e_{1} e_{2}+e_{0} e_{3}\right) & 2\left(e_{1} e_{3}-e_{0} e_{2}\right) \\
2\left(e_{1} e_{2}-e_{0} e_{3}\right) & e_{0}^{2}-e_{1}^{2}+e_{2}^{2}-e_{3}^{2} & 2\left(e_{2} e_{3}+e_{0} e_{1}\right) \\
2\left(e_{1} e_{3}+e_{0} e_{2}\right) & 2\left(e_{2} e_{3}-e_{0} e_{1}\right) & e_{0}^{2}-e_{1}^{2}-e_{2}^{2}+e_{3}^{2}
\end{array}\right]
$$

This is the matrix form of an element of $S O(3)$ in terms of its unit quaternion representation. This representation of rotation matrices in 3-dimensional spsce is often called the Euler parametrisation. The double representation can be avoided by taking $\left(e_{0}: e_{1}: e_{2}: e_{3}\right)$ as homogeneous coordinates for projective 3 -space. In that case, the scalar multiple $\left(e_{0}^{2}+e_{1}^{2}+e_{2}^{2}+e_{3}^{2}\right)^{-1}$ of (2.21) is required.

Planar rotations are not as complicated as spatial ones so trigonometric representation of the rotation matrix as in (2.15) is generally sufficient. Yet, there is the possibility of representing an element of $S O(2)$ by a unit quaternion. Assuming the $z$-axis to be the axis of rotation, we choose $e_{1}=e_{2}=0$. Thus, taking the leading $(2 \times 2)$ submatrix in $(2.21)$, a rotation matrix rep- 
resenting an element of $S O(2)$ in terms of the unit quaternion $e_{0}+e_{3} \mathrm{k}$ is

$$
\left[\begin{array}{cc}
e_{0}^{2}-e_{3}^{2} & 2 e_{0} e_{3} \\
-2 e_{0} e_{3} & e_{0}^{2}-e_{3}^{2}
\end{array}\right]
$$

\subsubsection{The special Euclidean groups}

We now show how to extend parametrisations for the orthogonal groups to the Euclidean groups. First, we start with Euler angles $\theta_{x}, \theta_{y}, \theta_{z} \in \mathbb{R}^{3}$ combined with a position vector $\mathbf{t} \in \mathbb{R}^{3}$ whose components are three linear translations $t_{x}, t_{y}, t_{z}$ along the axes of the coordinate system of $\mathbb{R}^{3}$. As a result, an element of $S E(3)$ can be thought of as a real 6-vector i.e. $\left(\theta_{x}, \theta_{y}, \theta_{z}, t_{x}, t_{y}, t_{z}\right) \in \mathbb{R}^{6}$. It is possible to write this representation as a $(4 \times 4)$ matrix whose shape is given in $(2.10)$, where $A$ is the rotation matrix in $(2.14)$

$$
\left[\begin{array}{cccc}
c_{x} c_{y} c_{z}-s_{x} s_{z} & -c_{y} s_{x}-c_{x} c_{y} s_{z} & c_{x} s_{y} & t_{x} \\
c_{y} c_{z} s_{x}+c_{x} s_{z} & c_{x} c_{z}-c_{y} s_{x} s_{z} s_{z} & s_{x} s_{y} & t_{y} \\
-c_{z} s_{y} & s_{y} s_{z} & c_{y} & t_{z} \\
0 & 0 & 0 & 1
\end{array}\right]
$$

Alternatively, we can employ a unit quaternion q (Euler parameters) instead of Euler angles. Following this replacement, an element of $S E(3)$ will be given by a 7 -vector i.e. $\left(e_{0}, e_{1}, e_{2}, e_{3} ; t_{x}, t_{y}, t_{z}\right) \in \mathbb{R}^{7}$ that we can still describe as a $(4 \times 4)$ matrix whose rotation part is given by $(2.21)$

$$
\left[\begin{array}{cccc}
e_{0}^{2}+e_{1}^{2}-e_{2}^{2}-e_{3}^{2} & 2\left(e_{1} e_{2}+e_{0} e_{3}\right) & 2\left(e_{1} e_{3}-e_{0} e_{2}\right) & t_{x} \\
2\left(e_{1} e_{2}-e_{0} e_{3}\right) & e_{0}^{2}-e_{1}^{2}+e_{2}^{2}-e_{3}^{2} & 2\left(e_{0} e_{1}+e_{2} e_{3}\right) & t_{y} \\
2\left(e_{0} e_{2}+e_{1} e_{3}\right) & 2\left(e_{2} e_{3}-e_{0} e_{1}\right) & e_{0}^{2}-e_{1}^{2}-e_{2}^{2}+e_{3}^{2} & t_{z} \\
0 & 0 & 0 & 1
\end{array}\right]
$$

We can easily deduce from (2.23) a way to represent a planar displace- 
ment. For translation only a 2-vector is required

$$
\mathbf{t}=\left(t_{x}, t_{y}\right) \in \mathbb{R}^{2}
$$

Therefore, an element of $S E(2)$ corresponding to a displacement in the $x y$ plane, is represented by a $(3 \times 3)$ matrix

$$
\left[\begin{array}{ccc}
c_{z} & -s_{z} & t_{x} \\
s_{z} & c_{z} & t_{y} \\
0 & 0 & 1
\end{array}\right]
$$

or with planar Euler parameters

$$
\left[\begin{array}{ccc}
e_{0}^{2}-e_{3}^{2} & 2 e_{0} e_{3} & t_{x} \\
-2 e_{0} e_{3} & e_{0}^{2}-e_{3}^{2} & t_{y} \\
0 & 0 & 1
\end{array}\right]
$$

Most simply, an element of $S E(2)$ can be parametrised by a 3-vector

$$
\left(\theta_{z}, t_{x}, t_{y}\right) \in \mathbb{R}^{3}
$$

Note, however, that $\left(\theta_{z}+2 \pi k, t_{x}, t_{y}\right)$ represent the same displacement for all integers $k$. These are three pose variables that are necessary and sufficient to express the position and orientation of a moving frame $M$ with respect to the reference frame $R$.

In the same way that quaternions provide an algebraic structure for describing rotations, Clifford [9] showed that there is an algebraic model for rigid displacements, which he called biquaternions. They are now usually known as dual quaternions. In the next sections we describe them and their connection to $S E(3)$. 


\subsubsection{Dual numbers}

Dual numbers were initially introduced by Clifford [9] as an extension of real numbers rather like complex numbers, and later used by Study [11] in describing the geometry of lines in space. A dual number $\breve{d} \in \mathbb{D}$ has the form $p+\varepsilon d$ where $p, d \in \mathbb{R}$ are called its primal and dual parts, respectively, and $\varepsilon$ is the dual unit satisfying $\varepsilon^{2}=0$. Addition on dual numbers is defined componentwise

$$
\left(p_{1}+\varepsilon d_{1}\right)+\left(p_{2}+\varepsilon d_{2}\right)=\left(p_{1}+p_{2}\right)+\varepsilon\left(d_{1}+d_{2}\right)
$$

while multiplication is defined distributively by

$$
\begin{aligned}
\left(p_{1}+\varepsilon d_{1}\right)\left(p_{2}+\varepsilon d_{2}\right) & =p_{1} p_{2}+\varepsilon p_{1} d_{2}+\varepsilon d_{1} p_{2}+\varepsilon^{2} d_{1} d_{2} \\
& =p_{1} p_{2}+\varepsilon\left(p_{1} d_{2}+d_{1} p_{2}\right)
\end{aligned}
$$

Since $\varepsilon^{2}=0$.

Give a dual number $\breve{d}=p+\varepsilon d$, its conjugate is defined by

$$
\breve{d}^{*}=p-\varepsilon d
$$

and $\breve{d} \breve{d}^{*}=p^{2}$. Therefore, if $p \neq 0$, the inverse of $\breve{d}$ is given by

$$
\breve{d}^{-1}=\frac{\breve{d}^{*}}{\breve{d} \breve{d}^{*}}=\frac{1}{p^{2}}(p-\varepsilon d)=p^{-1}\left(1-\varepsilon d p^{-1}\right)
$$

Note that if $p=0$, i.e. $\breve{d}$ is a pure dual, $\breve{d}$ has no inverse. It follows that $\mathbb{D}$ does not form a field but it is a ring, as well as a 2-dimensional real vector space.

We can also define a dual vector $\breve{\mathbf{v}}=\mathbf{v}+\varepsilon \mathbf{v}^{\prime} \in \mathbb{D}^{n}$ where $\mathbf{v}, \mathbf{v}^{\prime} \in \mathbb{R}^{n}$ and dual matrices. These are modules over the ring $\mathbb{D}$ or real vector spaces. We 
can define dot product and cross product on dual vectors

$$
\breve{\mathbf{u}} \cdot \breve{\mathbf{v}}=\mathbf{u} \cdot \mathbf{v}+\varepsilon\left(\mathbf{u} \cdot \mathbf{v}^{\prime}+\mathbf{u}^{\prime} \cdot \mathbf{v}\right)
$$

and for $n=3$

$$
\breve{\mathbf{u}} \times \breve{\mathbf{v}}=\mathbf{u} \times \mathbf{v}+\varepsilon\left(\mathbf{u}^{\prime} \times \mathbf{v}+\mathbf{u} \times \mathbf{v}^{\prime}\right)
$$

where and $\times$ on the right stand for real vector dot product and cross product.

\subsubsection{Dual quaternions and spatial displacement}

A dual quaternion is an object of the form

$$
\breve{\mathbf{q}}=\mathbf{q}_{p}+\varepsilon \mathbf{q}_{d} \in \mathbb{D} \mathbb{H}
$$

where $\mathbf{q}_{p}:=\left(e_{p^{0}}, \mathbf{e}_{p}\right)$ and $\mathbf{q}_{d}:=\left(e_{d^{0}}, \mathbf{e}_{d}\right)$ are quaternions, called the primal and dual, respectively. It can also be expressed in the form $\breve{\mathbf{q}}:=(\breve{s}, \breve{\mathbf{v}})=\breve{s}+\breve{\mathbf{v}}$ where $\breve{s}:=\left(q_{p^{0}}, q_{d^{0}}\right)=q_{p^{0}}+\varepsilon q_{d^{0}} \in \mathbb{D}$ is the dual scalar and $\breve{\mathbf{v}}:=\left(\mathbf{e}_{p}, \mathbf{e}_{d}\right)=$ $\mathbf{e}_{p}+\varepsilon \mathbf{e}_{d} \in \mathbb{D}^{3}$ is the dual vector. In addition, a dual quaternion $\breve{\mathbf{q}}$ can also be seen as an 8-vector space over real numbers

$$
\breve{\mathbf{q}}=\left(q_{p^{0}}, q_{p^{1}}, q_{p^{2}}, q_{p^{3}} ; q_{d^{0}}, q_{d^{1}}, q_{d^{2}}, q_{d^{3}}\right) \in \mathbb{R}^{8}
$$

whose basis consists of $\{1, \mathrm{i}, \mathrm{j}, \mathrm{k}, \varepsilon, \varepsilon \mathrm{i}, \varepsilon \mathrm{j}, \varepsilon \mathrm{k}\}$.

Addition on dual quaternions is done componentwise

$$
\breve{\mathbf{q}}+\breve{\mathbf{p}}=\left(\mathbf{q}_{p}+\mathbf{p}_{p}\right)+\varepsilon\left(\mathbf{q}_{d}+\mathbf{p}_{d}\right)
$$

while multiplication is given by

$$
\breve{\mathbf{q}} \breve{\mathbf{p}}=\mathbf{q}_{p} \mathbf{p}_{p}+\varepsilon\left(\mathbf{q}_{p} \mathbf{p}_{d}+\mathbf{q}_{d} \mathbf{p}_{p}\right)
$$


where quaternion multiplication on the right is given in (2.16). Dual quaternion multiplication can also be stated in following format which is sometimes more useful

$$
\breve{\mathbf{q}} \breve{\mathbf{p}}=\left(\breve{s}_{1}, \breve{\mathbf{v}}_{1}\right)\left(\breve{s}_{2}, \breve{\mathbf{v}}_{2}\right)=\left(\left(\breve{s}_{1} \breve{s}_{2}-\breve{\mathbf{v}}_{1} \cdot \breve{\mathbf{v}}_{2}\right),\left(\breve{s}_{1} \breve{\mathbf{v}}_{2}+\breve{s}_{2} \breve{\mathbf{v}}_{1}+\breve{\mathbf{v}}_{1} \times \breve{\mathbf{v}}_{2}\right)\right)
$$

which follows from (2.31) and (2.32).

Since a dual quaternion $\breve{\mathbf{q}}=\mathbf{q}_{p}+\varepsilon \mathbf{q}_{d}$ involves quaternions and dual numbers, there are three types of conjugations associated to it; namely quaternion conjugation as in (2.17), dual number conjugation as in (2.30), or a combination of both of these

- quaternion conjugation: $\breve{\mathbf{q}}^{*}=\mathbf{q}_{p}^{*}+\varepsilon \mathbf{q}_{d}^{*}$

- dual conjugation: $\breve{\mathbf{q}}=\mathbf{q}_{p}-\varepsilon \mathbf{q}_{d}$

- combination of both: $\breve{\mathbf{q}}^{\diamond}=\mathbf{q}_{p}^{*}-\varepsilon \mathbf{q}_{d}^{*}$

It turns out that this third type of conjugation is relevant for representing spatial displacement. Extending what we already discussed in Section 2.4.2 regarding the quaternion norm, we have

$$
\breve{\mathbf{q}} \breve{\mathbf{q}}^{*}=\breve{\mathbf{q}}^{*} \breve{\mathbf{q}}=\left\|\mathbf{q}_{p}\right\|^{2}+\varepsilon 2 \mathbf{q}_{p} \cdot \mathbf{q}_{d}
$$

We can regard this as a type of (squared) norm for a dual quaternion.

A dual quaternion $\breve{\mathbf{q}}$ with $\breve{\mathbf{q}} \breve{\mathbf{q}}^{*}=1$ is called a unit dual quaternion. By (2.37), this is equivalent to the two conditions

$$
\begin{array}{r}
\left\|\mathbf{q}_{p}\right\|^{2}=q_{p^{0}}^{2}+q_{p^{1}}^{2}+q_{p^{2}}^{2}+q_{p^{3}}^{2}=1 \\
\mathbf{q}_{p} \cdot \mathbf{q}_{d}=q_{p^{0}} q_{d^{0}}+q_{p^{1}} q_{d^{1}}+q_{p^{2}} q_{d^{2}}+q_{p^{3}} q_{d^{3}}=0
\end{array}
$$

Accordingly, for a unit dual quaternion $\breve{\mathbf{q}}$, the primal must be a unit quaternion belonging to $S^{3}$, while the dual must be perpendicular to it with respect 
to the standard dot product on $\mathbb{R}^{4}$. In effect, $\mathbf{q}_{d}$ is a tangent vector to $S^{3}$ at $\mathbf{q}_{p}$ so that the unit dual quaternions, denoted $\mathbb{D} S^{3}$, form the tangent bundle to $S^{3}$. Besides, they are a group under multiplication. In fact, it is a Lie group (for more details see [70] Section 9.3). Equations (2.38a) and (2.38b) impose two conditions on the eight variables so it is 6-dimensional.

The use of unit dual quaternions to represent spatial displacements extends the idea of conjugation that we discussed for spatial rotations. We now regard the space $\mathbb{R}^{3}$ as the dual imaginary quaternions with primal part the identity i.e. $1+\varepsilon \mathbf{v} \in \mathbb{D} \mathbb{H}$ where $\mathbf{v}=v_{1} \mathrm{i}+v_{2} \mathbf{j}+v_{3} \mathrm{k} \in \mathbb{H}$. Suppose $\breve{\mathbf{q}}=\mathbf{q}_{p}+\varepsilon \mathbf{q}_{d}$ is a unit dual quaternion, then

$$
\begin{aligned}
\breve{\mathbf{q}}(1+\varepsilon \mathbf{v}) \breve{\mathbf{q}}^{\diamond} & =\left(\mathbf{q}_{p}+\varepsilon \mathbf{q}_{d}\right)(1+\varepsilon \mathbf{v})\left(\mathbf{q}_{p}^{*}-\varepsilon \mathbf{q}_{d}^{*}\right) \\
& =\mathbf{q}_{p} \mathbf{q}_{p}^{*}+\varepsilon\left(\mathbf{q}_{p} \mathbf{v} \mathbf{q}_{p}^{*}+\mathbf{q}_{d} \mathbf{q}_{p}^{*}-\mathbf{q}_{p} \mathbf{q}_{d}^{*}\right) \\
& =1+\varepsilon\left(\mathbf{q}_{p} \mathbf{v} \mathbf{q}_{p}^{*}+\mathbf{t}\right)
\end{aligned}
$$

where $\mathbf{t}=2 \operatorname{Im}\left(\mathbf{q}_{d} \mathbf{q}_{p}^{*}\right)$ is a pure quaternion. Since $\mathbf{q}_{p} \in S^{3}$, the dual part represents rotation followed by translation by $\mathbf{t}$ in $\mathbb{R}^{3}$. The choice $\mathbf{q}_{d}=\frac{1}{2} \mathbf{t} \mathbf{q}_{p}$ gives translation $\mathbf{t}$. It is straightforward to check that

$$
\begin{aligned}
\left(\mathbf{q}_{p}+\frac{1}{2} \varepsilon \mathbf{t} \mathbf{q}_{\mathbf{p}}\right)\left(\mathbf{q}_{p}+\frac{1}{2} \varepsilon \mathbf{t} \mathbf{q}_{\mathbf{p}}\right)^{*} & =\mathbf{q}_{p} \mathbf{q}_{p}^{*}+\frac{1}{2} \varepsilon\left(\mathbf{q}_{p} \mathbf{q}_{p}^{*} \mathbf{t}^{*}+\mathbf{t} \mathbf{q}_{p} \mathbf{q}_{p}^{*}\right) \\
& =1+\frac{1}{2} \varepsilon\left(\mathbf{t}^{*}+\mathbf{t}\right) \\
& =1
\end{aligned}
$$

since $\mathbf{t}^{*}=-\mathbf{t}$ for a pure quaternion, so that $\mathbf{q}_{p}+\varepsilon \mathbf{q}_{d} \in \mathbb{D} S^{3}$. Since $\mathbf{q}_{p},-\mathbf{q}_{p} \in$ $S^{3}$ represent the same spatial rotation in $S O(3)$, it follows that $\breve{\mathbf{q}},-\breve{\mathbf{q}} \in \mathbb{D} S^{3}$ represent the same spatial rigid displacement in $S E(3)$. In other words, there is a $2: 1$ homomorphism $\mathbb{D} S^{3} \rightarrow S E(3)$ (for more details see [70] Page 211).

As we showed for quaternions, sometimes, it is more convenient if we write a dual quaternion $\breve{\mathbf{q}}$ in a matrix form [83, 84]. A dual quaternion 
$\breve{\mathbf{q}}=\mathbf{q}_{p}+\varepsilon \mathbf{q}_{d}$ can be rewritten as the following $(8 \times 8)$ matrix

$$
\breve{Q}:=\left[\begin{array}{ll}
Q_{p} & 0_{4} \\
Q_{d} & Q_{p}
\end{array}\right]
$$

where $Q_{p}$ and $Q_{d}$ are the $(4 \times 4)$ matrices representing $\mathbf{q}_{p}, \mathbf{q}_{d}$, respectively, as in (2.20). As expected, dual quaternion multiplication corresponds to matrix multiplication in this representation

$$
\breve{Q} \breve{P}=\left[\begin{array}{ll}
Q_{p} & 0_{4} \\
Q_{d} & Q_{p}
\end{array}\right]\left[\begin{array}{cc}
P_{p} & 0_{4} \\
P_{d} & P_{p}
\end{array}\right]=\left[\begin{array}{cc}
Q_{p} P_{p} & 0_{4} \\
Q_{d} P_{p}+Q_{p} P_{d} & Q_{p} P_{p}
\end{array}\right]
$$

Note that $Q_{p} P_{p}$ can be thought of as the matrix form of (2.16).

In a matrix form, the point $\mathbf{v}=\left(v_{1}, v_{2}, v_{3}\right) \in \mathbb{R}^{3}$, with dual quaternion form $1+\varepsilon \mathbf{v}$ is given by

$$
\breve{V}:=\left[\begin{array}{cc}
I_{4} & 0_{4} \\
V & I_{4}
\end{array}\right]
$$

where

$$
V=\left[\begin{array}{cccc}
0 & -v_{1} & -v_{2} & -v_{3} \\
v_{1} & 0 & -v_{3} & v_{2} \\
v_{2} & v_{3} & 0 & -v_{1} \\
v_{3} & -v_{2} & v_{1} & 0
\end{array}\right]
$$

and its rigid displacement by $\breve{\mathbf{q}}=\mathbf{q}_{p}+\varepsilon \mathbf{q}_{d}$ has matrix form

$$
\begin{aligned}
\breve{Q} \breve{V} \breve{Q}^{\diamond} & =\left[\begin{array}{ll}
Q_{p} & 0_{4} \\
Q_{d} & Q_{p}
\end{array}\right]\left[\begin{array}{cc}
I_{4} & 0_{4} \\
V & I_{4}
\end{array}\right]\left[\begin{array}{cc}
Q_{p}^{*} & 0_{4} \\
-Q_{d}^{*} & Q_{p}^{*}
\end{array}\right] \\
& =\left[\begin{array}{cc}
I_{4} & 0_{4} \\
Q_{d} Q_{p}^{*}+Q_{p} V Q_{p}^{*}-Q_{p} Q_{d}^{*} & I_{4}
\end{array}\right]
\end{aligned}
$$

Finally, we are able to find a dual quaternion parametrisation for a planar displacement although we already have a straightforward 3-parameter form 
$\left(\theta_{z}, t_{x}, t_{y}\right)$. The rotation is given by a unit quaternion of the form $\mathbf{q}_{p}=e_{0}+e_{3} \mathrm{k}$ as in (2.22). To represent the translation $\mathbf{t}=\left(t_{x}, t_{y}, 0\right)$, we require

$$
\begin{aligned}
\mathbf{q}_{d} & =\frac{1}{2} \mathbf{t q}_{p} \\
& =\frac{1}{2}\left(\left(e_{0} t_{x}+e_{3} t_{y}\right) \mathrm{i}+\left(e_{0} t_{y}-e_{3} t_{x}\right) \mathbf{j}\right)
\end{aligned}
$$

This gives the homogeneous matrix form for an element of $S E(2)$

$$
\left[\begin{array}{ccc}
e_{0}^{2}-e_{3}^{2} & 2 e_{0} e_{3} & \frac{1}{2}\left(e_{0} t_{x}+e_{3} t_{y}\right) \\
-2 e_{0} e_{3} & e_{0}^{2}-e_{3}^{2} & \frac{1}{2}\left(e_{0} t_{y}-e_{3} t_{x}\right) \\
0 & 0 & 1
\end{array}\right]
$$

\subsection{The pose space}

So far, we have discussed how a free displacement of a single rigid body can be described by a set of pose variables which specify an element of the Euclidean group $g \in S E(n), n=2,3$. Now a series of questions is raised: how we describe relative motion if two rigid components are jointed together, what is the impact if one of them is fixed, and what if a sequence or set of connected components is given and we seek to describe the relative or absolute displacement of each?

Let us start with two rigid links $L_{-}, L_{+}$, each assigned a moving frame, respectively $M_{-}, M_{+}$, that are connected together by a joint $J$. Note that ,-+ nominally denote the preceding and the following components/moving frames, respectively. However, there is not necessarily any natural ordering between the links. These two components move freely in the ambient space, which carries a reference frame $R$ as defined in Section 2.3. Displacement of each link relative to the ambient space is given by $g_{-}, g_{+} \in S E(n), n=2,3$. In effect, they form a 2-body system whose displacement is described by the 
pair of elements

$$
\left(g_{-}, g_{+}\right) \in S E(n) \times S E(n)
$$

This is a set of all pose variables that expresses the absolute coordinates (absolute displacements) of the system with respect to the ambient space.

The significance of the joint is that it imposes constraints on the pose variables. We describe this in Chapter 4. The motivation here is that a robot device or manipulator consists of rigid bodies that, in the absence of joints, are free to move independently in the ambient space. The introduction of joints imposes constraints on the relative motions. However, if there is not assumed to be a base - one rigid component fixed in the ambient space - then the bodies are additionally able to move in unison freely within the ambient space.

To model the relative displacement between a pair of links, consider the simultaneous (right) action of $S E(n)$ on the product $S E(n) \times S E(n)$

$$
\left(g_{-}, g_{+}\right) \circ h=\left(g_{-} \circ h, g_{+} \circ h\right)
$$

The quotient space $[S E(n) \times S E(n)] / S E(n)$ is homogeneous and represents the residual relative displacement. In practice, we can identify one of the moving frames (e.g. $M_{-}$) with the reference frame $R$, by choosing $h=g_{-}^{-1}$. So every element of the quotient $\left[\left(g_{-}, g_{+}\right)\right]$, denoting the coset of $\left(g_{-}, g_{+}\right)$, can be represented by

$$
(e, g) \in[S E(n) \times S E(n)] / S E(n)
$$

where $g=g_{+} g_{-}^{-1}$, giving an identification of the quotient space with $S E(n)$. Although this element still involves pose variables, it only represents the relative displacements of $M_{+}$with respect to $M_{-}$.

If a set of $k+1$ rigid bodies $L_{i}, i=0, \ldots, k$, constrained together in pairs via $t$ joints $J_{j}, j=1, \ldots, t$ is considered, then there will be a product 
of $k+1$ copies of $S E(n)$ to be taken into account. This describes possible displacements of a $(k+1)$-body system in terms of pose variables for $g_{i}, i=$ $0, \ldots, k$. The relative displacements of the links are, as for a pair of links, represented by the homogeneous space

$$
[S E(n)]^{k+1} / S E(n)
$$

Equivalently, we can assume one link, say $L_{0}$, to be fixed such that the rest of the system has six or fewer degrees of freedom. This space, called the pose space (or M-space) is isomorphic to a product of $k$ copies of $S E(n)$

$$
\mathcal{M}:=[S E(n)]^{k}
$$

Its dimension is $m=k \times p$, where $p$ is as defined in (2.9). Note that the Mspace can be locally parametrised by $m$-dimensional Euclidean space. Thus, we may sometimes think of the $\mathcal{M}$ as the Euclidean space $\mathbb{R}^{m}$ where there is no confusion likely. Alternatively, we may use a parametrisation, such as by dual quaternions (Section 2.4.5) with more parameters requiring additional equations such as (2.38a) and (2.38b).

In the next chapter, we will explore the tangent spaces of the Euclidean group and in particular the Lie algebra associated to that group. Line geometry will be studied, as a central core, and will be followed by screw theory as a powerful tool to represent the Lie algebra. 


\section{Chapter 3}

\section{The Euclidean Lie algebra \& associated geometry}

A very nice aspect of Lie theory is that a Lie group $G$, which is usually a nonlinear (curved) smooth manifold, can be analysed and studied to a significant extent by considering a linear (flat) space associated to it. This linear object is the tangent space of the group at its identity element, $T_{e} G$. This tangent space inherits the binary operation from the group and is called the Lie algebra $\mathfrak{g}$ associated to the group $G$. The Lie algebra can be thought of as infinitesimal elements of the group $G$.

Having considered the group $G$ to be a smooth manifold, its associated tangent space is made up of tangent vectors to all smooth paths $\sigma(t)$ in $G$ such that $\sigma(0)=e$. Thus, a tangent vector is defined as the first-order derivative at the identity $\dot{\sigma}(0)=\frac{d}{d t}[\sigma(t)]_{t=0}$.

When, in the $19^{\text {th }}$ century, Sir Robert S. Ball [10] developed his theory of screws and defined a twist as the canonical motion of a rigid body, he also noticed that a screw can carry an instantaneous twist-effectively an infinitesimal element of the special Euclidean group $S E(3)$. This is right because we can think of a tangent vector to represent a velocity at the identity. Yet, both points of view are fine and true, the only matter is that Ball's 
perspective was rather like that of Sophus Lie in the more general setting of Lie groups.

In this Chapter, we shall introduce the Lie algebras for the groups considered in Chapter 2 and give several representations of them. We also show how they relate to Screw theory.

\subsection{Tangent spaces \& Lie algebras}

In general, a Lie group is an abstract differentiable manifold as described in Chapter 2. However, the groups we are interested in - the orthogonal and Euclidean groups - can be thought of as groups of matrices. Therefore, a group-valued function of real variables is smooth if and only if each matrix entry is a smooth function of its variables.

Consider a mapping $\sigma: \mathbb{R} \rightarrow G$ which corresponds to a smooth path ${ }^{1}$ in a Lie group $G$ through an element $g \in G$ i.e. $\sigma(0)=g$. Its derivative at $g$ is called a tangent vector $\zeta:=\frac{d}{d t}[\sigma(t)]_{t=0}$.

Assume two different smooth paths of this type $-\sigma_{1}, \sigma_{2}$. These paths are equivalent as long as their tangent vectors at $t=0-\zeta_{1}, \zeta_{2}$-are identical i.e. $\dot{\sigma}_{1}(0)=\dot{\sigma}_{2}(0)$. Consequently, it is right to think of the tangent space to be a set of all equivalent classes of smooth paths. However, when $G$ is a matrix Lie group, the tangent vectors $\zeta_{1}, \zeta_{2}$ are themselves matrices and it is necessary to know what properties they satisfy.

Denote the set of all tangent vectors $\zeta$ at $g \in G$ by $T_{g} G$. This is called the tangent space to $G$ at $g$ [76]. It is a (real) vector space of the same dimension as the Lie group $G$ itself. In particular, when $g=e$, the identity in $G$, denote the tangent space $T_{e} G$ by $\mathfrak{g}$. Suppose $\zeta, \eta \in \mathfrak{g}$ and that $\sigma, \tau: \mathbb{R} \rightarrow G$ are paths that represent them, so $\sigma(0)=\tau(0)=e$ and $\dot{\sigma}(0)=\zeta$ and $\dot{\tau}(0)=\eta$. For fixed $s \in \mathbb{R}$, the function $t \mapsto \sigma(s) \tau(t) \sigma^{-1}(s)$ is a smooth path through

\footnotetext{
${ }^{1} \mathrm{~A}$ path is smooth as long as its derivatives of all order exist and are continuous.
} 
$e \in G$ at $t=0$ so that

$$
\frac{d}{d t}\left[\sigma(s) \tau(t) \sigma^{-1}(s)\right]_{t=0}=\sigma(s) \eta \sigma^{-1}(s) \in \mathfrak{g}
$$

This gives a smooth path in $\mathfrak{g}$ and, since it is a vector space, its derivative is also in $\mathfrak{g}$

$$
\frac{d}{d s}\left[\sigma(s) \eta \sigma^{-1}(s)\right]_{s=0}=\zeta \eta-\eta \zeta
$$

This commutator of $\zeta$ and $\eta$ is denoted by $[\zeta, \eta]$, and called the Lie bracket of $\zeta, \eta$. The tangent space $\mathfrak{g}$, together with this binary operation, is called a Lie algebra. The Lie bracket is bilinear, anti-symmetric and satisfies a further property called the Jacobi identity, which we will not require. See any standard textbook, such as [76] for more details.

\subsection{Lie algebra of $S O(3)$}

We already discussed that an element $g \in S O(3)$ is represented by $(3 \times 3)$ rotation matrix $A$ as in (2.14). Consider a smooth path associated to $S O(3)$ i.e. $A: \mathbb{R} \rightarrow S O(3)$ such that $A(0)=I_{3}$ and thus $A(t)^{T} A(t)=I_{3}$ for all $t \in \mathbb{R}$. Differentiating this equality with respect to $t$ and evaluating it at $t=0$ gives

$$
\left[\frac{d}{d t} A(t)^{T} A(t)+A(t)^{T} \frac{d}{d t} A(t)\right]_{t=0}=0_{3}
$$

From now on, $0_{n}$ stands for the $n$-dimensional zero matrix. Since $[A(t)]_{t=0}=$ $\left[A(t)^{T}\right]_{t=0}=I_{3}$, we can define $\Omega=\frac{d}{d t}[A(t)]_{t=0}=\dot{A}(0)$. Thus, equation 3.3 can be written as

$$
\Omega^{T}+\Omega=0_{3}
$$

and so $\mathfrak{s o}(3)=T_{e} S O(3):=\left\{\Omega \in G L(3, \mathbb{R}): \Omega^{T}=-\Omega\right\}$. That is, $\Omega$ is a skew-symmetric matrix 


$$
\Omega:=\left[\begin{array}{ccc}
0 & -\omega_{z} & \omega_{y} \\
\omega_{z} & 0 & -\omega_{x} \\
-\omega_{y} & \omega_{x} & 0
\end{array}\right]
$$

and confirms that $\mathfrak{s o}(3)$ is a 3 -dimensional vector space spanned by the three matrices

$$
\mathrm{I}=\left[\begin{array}{ccc}
0 & 0 & 0 \\
0 & 0 & -1 \\
0 & 1 & 0
\end{array}\right], \quad \mathrm{J}=\left[\begin{array}{ccc}
0 & 0 & 1 \\
0 & 0 & 0 \\
-1 & 0 & 0
\end{array}\right], \quad \mathrm{K}=\left[\begin{array}{ccc}
0 & -1 & 0 \\
1 & 0 & 0 \\
0 & 0 & 0
\end{array}\right]
$$

These correspond to infinitesimal rotations about three unit vectors $\mathrm{i}, \mathrm{j}, \mathrm{k}$ in the Cartesian coordinate system arising from the paths defined in (2.13).

Suppose $\mathbf{p} \in \mathbb{R}^{3}, \mathbf{p} \neq \mathbf{0}$ and consider the path $\mathbf{P}(t)=A(t) \mathbf{p} \in \mathbb{R}^{3}$, which satisfies $\mathbf{P}(0)=\mathbf{p}$. Its velocity at $t=0$ is $\dot{\mathbf{P}}(0)=\dot{A}(0) \mathbf{p}=\Omega \mathbf{p}$. Since $A(t)$ is orthogonal, $\|\mathbf{P}(t)\|^{2}$ is constant, so that $\dot{\mathbf{P}}(0)$ is orthogonal to $\mathbf{p}$. Moreover, it is easy to check that

$$
\Omega \mathbf{p}=\omega \times \mathbf{p}
$$

where $\boldsymbol{\omega}=\left(\omega_{x}, \omega_{y}, \omega_{z}\right)^{T} \in \mathbb{R}^{3}$ and $\times$ is the vector (cross) product in $\mathbb{R}^{3}$. For this reason some authors write $\Omega$ as " $\boldsymbol{\omega} \times$ ". Physically, the vector $\boldsymbol{\omega}$ represents the angular velocity of a point $\mathbf{p}$ moving along the path $\mathbf{P}(t)$ at $t=0$ or, equivalently, of a moving frame $M$ of rigid body rotating by $A(t)$.

More generally, if $A(t)$ is a path in the special orthogonal group with $A_{0}:=A(0) \in S O(3)$ then similarly to (3.3)

$$
\dot{A}_{0}^{T} A_{0}+A_{0}^{T} \dot{A}_{0}=0_{3}
$$

where $\dot{A}_{0}:=\dot{A}(0)$. It follows that $A_{0}^{T} \dot{A}_{0}=\Omega_{L}$ is skew-symmetric. This defines a function $T_{A_{0}} S O(3) \rightarrow \mathfrak{s o}(3), \dot{A}_{0} \mapsto \Omega_{L}$ called the left pullback. Likewise, the right pullback is $\dot{A}_{0} \mapsto \Omega_{R}=\dot{A}_{0} A_{0}^{T}$. In terms of coordinate 
frames for a rigid body, these pullbacks correspond to a change of moving frame $M$ so that it (instantaneously) coincides with the reference frame $R$ left pullback - or the other way around-right pullback. We discuss this further is Section 3.3.

We saw in Section 2.4.2 the close relation between unit quaternions, $S^{3}$, and the rotation group $S O(3)$. Since the relationship is $2: 1$, it provides an isomorphism between their Lie algebras. The condition $\mathbf{q q}^{*}=e_{0}^{2}+e_{1}^{2}+e_{2}^{2}+$ $e_{3}^{2}=1$ means that for a path $\mathbf{q}: \mathbb{R} \rightarrow S O(3), \mathbf{q}(0)=1,2 \operatorname{Re}\left(\dot{\mathbf{q}} \mathbf{q}^{*}\right)=\mathbf{q} \cdot \dot{\mathbf{q}}=0$ where the product is the usual scalar product on $\mathbb{R}^{4}$

$$
\begin{aligned}
\frac{d}{d t}\left[\mathbf{q}(t) \mathbf{q}^{*}(t)\right]_{t=0} & =\left[\dot{\mathbf{q}}(t) \mathbf{q}^{*}(t)+\mathbf{q}(t) \dot{\mathbf{q}}(t)\right]_{t=0} \\
& =\dot{\mathbf{q}} \mathbf{q}^{*}+\left(\dot{\mathbf{q}} \mathbf{q}^{*}\right)^{*} \\
& =2 \operatorname{Re}\left(\dot{\mathbf{q}} \mathbf{q}^{*}\right) \\
& =2 \operatorname{Re}\left[\left(\dot{e}_{0}+\dot{e}_{1} \mathrm{i}+\dot{e}_{2} \mathrm{j}+\dot{e}_{3} \mathrm{k}\right)\left(e_{0}-e_{1} \mathrm{i}-e_{2} \mathrm{j}-e_{3} \mathrm{k}\right)\right] \\
& =2\left[e_{0} \dot{e}_{0}+e_{1} \dot{e}_{1}+e_{2} \dot{e}_{2}+e_{3} \dot{e}_{3}\right] \\
& =\mathbf{q} \cdot \dot{\mathbf{q}}
\end{aligned}
$$

It follows that the tangent space $T_{1} S^{3}$ consists of the pure imaginary quaternions and is spanned by $\mathrm{i}, \mathrm{j}, \mathrm{k}$. These correspond to the infinitesimal rotations in $(3.6)$.

In Section 2.4.1, we treated $S O(2)$ as a subset of $S O(3)$ by fixing the axis of rotation as the $z$-axis. The Lie algebra $\mathfrak{s o}(2)$ then corresponds to the 1-dimensional subalgebra of $\mathfrak{s o}(3)$ spanned by the matrix $\mathrm{K}$ in (3.6). We can represent elements by the $(2 \times 2)$ matrix

$$
\left[\begin{array}{cc}
0 & -w_{z} \\
w_{z} & 0
\end{array}\right]
$$




\subsection{Lie algebra of $S E(3)$}

In this section, we study the Lie algebra of the 3-dimensional Euclidean group which represents a general rigid displacement which is undertaken by a rotation about an axis through the origin followed by a translation - that is $S E(3) \cong S O(3) \ltimes \mathbb{R}^{3}$. It is worth noting that the theorem of MozziChasles [63, 64] gives a different but equivalent way of describing a general rigid displacement (for proof and more discussion, see [16] Page 49 and [70] Section 2.5.2). Note, however, that for a pure translation the axis is "at infinity". This description is often called a screw displacement, or finite screw.

Theorem 3.1 (Mozzi-Chasles Theorem). Every spatial displacement is the composition of a rotation about an axis - a line anywhere in $\mathbb{R}^{3}$-together with a translation along that axis.

Consider rigid body displacement parametrised by $s$, that is a smooth path $\sigma:=(A, \mathbf{t})(s): \mathbb{R} \rightarrow S E(3)$, which in the homogeneous representation has the form

$$
\sigma(s)=\left[\begin{array}{cc}
A(s) & \mathbf{t}(s) \\
0 & 1
\end{array}\right]
$$

and suppose $A(0)=I_{3}, \mathbf{t}(0)=\mathbf{0}$. Differentiation of $\sigma$ with respect to $s$ yields

$$
\dot{\sigma}(s)=\left[\begin{array}{cc}
\dot{A}(s) & \dot{\mathbf{t}}(s) \\
0 & 0
\end{array}\right] \in T_{\sigma(s)} S E(3)
$$

Equating (3.9) at $s=0$ will provide us with an element of Lie algebra $\mathfrak{s e ( 3 )}$ which is called a twist

$$
X:=\left[\begin{array}{cc}
\Omega & \mathbf{v} \\
0 & 0
\end{array}\right] \in \mathfrak{s e}(3)
$$

where $\Omega$ is the $(3 \times 3)$ skew-symmetric matrix given in (3.5) whilst $\mathbf{v}=$ $\left(v_{x}, v_{y}, v_{z}\right)^{T} \in \mathbb{R}^{3}$. As it is explained in Section 3.2, a skew-symmetric matrix $\Omega \in G L(3, \mathbb{R})$ can be rewritten as a 3 -vector $\boldsymbol{\omega} \in \mathbb{R}^{3}$. Accordingly, it is often 
convenient to write a twist given in (3.10) in the vector form

$$
\boldsymbol{\xi}:=(\boldsymbol{\omega} ; \mathbf{v})^{T}=\left(\omega_{x}, \omega_{y}, \omega_{z} ; v_{x}, v_{y}, v_{z}\right) \in \mathbb{R}^{6}
$$

In Section 2.4.5, we showed how rigid displacements in $S E(3)$ can be represented by dual quaternions $\breve{\mathbf{q}}=\mathbf{q}_{p}+\varepsilon \mathbf{q}_{d}, \mathbf{q}_{p}, \mathbf{q}_{d} \in \mathbb{H}$, satisfying the two conditions (2.38a) and (2.38b). The associated group $\mathbb{D} S^{3}$, like its non-dual version $S^{3}$, has as its Lie algebra the pure imaginary (or vectorial) dual quaternions $\boldsymbol{\omega}+\varepsilon \mathbf{v}$ where

$$
\boldsymbol{\omega}=\omega_{1} \mathrm{i}+\omega_{2} \mathrm{j}+\omega_{3} \mathrm{k}, \quad \mathbf{v}=v_{1} \mathrm{i}+v_{2} \mathrm{j}+v_{3} \mathrm{k}
$$

Of course, we can represent these as 6 -vectors $(\boldsymbol{\omega} ; \mathbf{v})$. This provides another way to represent twists. We return to this in Section 3.4.2.

A twist as in (3.10) is also called instantaneous screw (see, for example, [10]) because it describes the infinitesimal motion along the screw axis arising from a 1-parameter screw displacement in $\mathbb{R}^{3}$. We will explore this in more detail in Section 3.4.3.

Suppose we are interested in the velocity of an arbitrary point attached to a moving rigid body with moving frame $M$ and reference frame $R$. Assume, its body coordinates are given as $\mathbf{p}=\left(p_{x}, p_{y}, p_{z}\right)^{T} \in M$. If a smooth displacement given in (3.8) acts on this body, its coordinates in $R$ at time $s$ will be

$$
\mathbf{P}(s)=\sigma(s) \mathbf{p}=A(s) \mathbf{p}+\mathbf{t}(s)
$$

Differentiating (3.12) with respect to $s$, we can obtain the infinitesimal displacement of the point in the ambient space

$$
\dot{\mathbf{P}}(s)=\dot{A}(s) \mathbf{p}+\dot{\mathbf{t}}(s)
$$


(3.12) allows us to rewrite (3.13) as

$$
\dot{\mathbf{P}}(s)=\dot{\sigma}(s) \sigma^{-1}(s) \mathbf{P}
$$

By using the homogeneous representation in (2.10) and (3.5), we can express $\dot{\sigma} \sigma^{-1}$ in the following matrix form

$$
\left[\begin{array}{cc}
\dot{A} & \dot{\mathbf{t}} \\
0 & 0
\end{array}\right]\left[\begin{array}{cc}
A^{-1} & -A^{-1} \mathbf{t} \\
0 & 1
\end{array}\right]=\left[\begin{array}{cc}
\dot{A} A^{-1} & -\dot{A} A^{-1} \mathbf{t}+\dot{\mathbf{t}} \\
0 & 0
\end{array}\right]
$$

By comparing (3.14) with what we already obtained in (3.7), it can be immediately confirmed that $\dot{A} A^{-1}=\Omega_{R} \in \mathfrak{s o}(3)$ is the right pullback. In this case, the velocity of $\mathbf{P}$ can be obtained by

$$
\dot{\mathbf{P}}=\Omega_{R} \mathbf{P}+\mathbf{v}
$$

where $\mathbf{v}=\dot{\mathbf{t}}-\Omega_{R} \mathbf{t}$.

\subsubsection{Adjoint representation}

Following our discussion in Section 2.4.5 on matrix representation for $S E(3)$ in terms of dual quaternions, and pullback in the previous section, we now explain the adjoint representation of a group in which the result of the conjugation by a group element on its Lie algebra is represented. Suppose $G$ is a matrix Lie group and $h \in G$. Conjugation in $G$ by $h$ is the operation $g \mapsto h g h^{-1}$. Clearly, conjugation fixes the identity $e \in G$ so the derivative of this map (with respect to $g$ ) at the identity is a linear map $\mathfrak{g} \rightarrow \mathfrak{g}$ on the Lie algebra.

If $\zeta \in \mathfrak{g}$ and $\zeta=\dot{\sigma}(0)$ for a smooth path $\sigma: \mathbb{R} \rightarrow G, \sigma(0)=e$, then the derivative is effectively given by (3.1), that is

$$
\zeta \mapsto h \zeta h^{-1}
$$


This makes sense so long as elements of $G$, and hence $\mathfrak{g}$, can be written as matrices. This is called the adjoint representation of $G$ and we write $\operatorname{Ad}_{h} \zeta:=h \zeta h^{-1}$. For each $h \in G$, this is a linear map $\mathfrak{g} \rightarrow \mathfrak{g}$ and can be written in matrix form with respect to a choice of basis for $\mathfrak{g}$.

Specifically, for the Euclidean group $S E(3)$, consider an element $h:=$ $(A, \mathbf{t}) \in S E(3)$ whose inverse is given by $h^{-1}:=\left(A^{T},-A^{T} \mathbf{t}\right) \in S E(3)$. Consider a twist $X \in \mathfrak{s e}(3)$ given in (3.10), using homogeneous representation and (3.6), we can write

$$
\begin{aligned}
\operatorname{Ad}_{h} X & =h X h^{-1} \\
& =\left[\begin{array}{ll}
A & \mathbf{t} \\
0 & 1
\end{array}\right]\left[\begin{array}{ll}
\Omega & \mathbf{v} \\
0 & 0
\end{array}\right]\left[\begin{array}{cc}
A^{T} & -A^{T} \mathbf{t} \\
0 & 1
\end{array}\right] \\
& =\left[\begin{array}{cc}
A \Omega A^{T} & A \mathbf{v}-A \Omega A^{T} \mathbf{t} \\
0 & 0
\end{array}\right]
\end{aligned}
$$

We can convert this into a different form in terms of the vector form $\boldsymbol{\xi}=$ $(\boldsymbol{\omega} ; \mathbf{v})$ in (3.7). By doing a little bit of algebraic manipulation, it is possible to show that $A \Omega A^{T}$ has vector form $A \boldsymbol{\omega}$ and then $-A \Omega A^{T} \mathbf{t}=-(A \boldsymbol{\omega}) \times \mathbf{t}=$ $T A \boldsymbol{\omega}$ where $T \equiv \mathbf{t} \times$ is the skew-symmetric matrix corresponding to $\mathbf{t}$. As a result, it is possible to rewrite (3.17) in the following matrix form

$$
\operatorname{Ad}_{h}(\boldsymbol{\omega} ; \mathbf{v})=\left[\begin{array}{cc}
A & 0 \\
T A & A
\end{array}\right]\left[\begin{array}{l}
\boldsymbol{\omega} \\
\mathbf{v}
\end{array}\right]
$$

Clearly, this $(6 \times 6)$ matrix is indeed another representation of the rigid displacement $h=(A, \mathbf{t})$.

It is worth noting that if $G$ is a matrix Lie group, $h \in G$, and $\xi \in T_{h} G$, then left and right pullbacks $\xi_{L}=h^{-1} \xi, \xi_{R}=\xi h^{-1}$ in the Lie algebra $\mathfrak{g}$ are related by the adjoint representation

$$
\operatorname{Ad}_{h} \xi_{L}=h\left(h^{-1} \xi\right) h^{-1}=\xi h^{-1}=\xi_{R}
$$


That means that important geometric features of an infinitesimal displacement or twist are those invariant under adjoint action.

From (3.18), the adjoint action of $(A, \mathbf{t}) \in S E(3)$ on a twist $(\boldsymbol{\omega} ; \mathbf{v})$ gives

$$
(A \boldsymbol{\omega} ; T A \boldsymbol{\omega}+A \mathbf{v}) \in \mathfrak{s e}(3)
$$

Since $A \in S O(3)$

$$
\langle A \boldsymbol{\omega}, A \boldsymbol{\omega}\rangle=\langle\boldsymbol{\omega}, \boldsymbol{\omega}\rangle
$$

so that the inner product $\boldsymbol{\omega} \cdot \boldsymbol{\omega}$ is invariant. Also, we saw that $T A \boldsymbol{\omega}=\mathbf{t} \times A \boldsymbol{\omega}$ which is orthogonal to $A \boldsymbol{\omega}$, so $\langle A \boldsymbol{\omega}, T A \boldsymbol{\omega}\rangle=0$. Hence,

$$
\begin{aligned}
\langle A \boldsymbol{\omega}, T A \boldsymbol{\omega}+A \mathbf{v}\rangle & =\langle A \boldsymbol{\omega}, A \mathbf{v}\rangle \\
& =\langle\boldsymbol{\omega}, \mathbf{v}\rangle
\end{aligned}
$$

so that the inner product $\boldsymbol{\omega} \cdot \mathbf{v}$ is also invariant. These two invariants $\boldsymbol{\omega} \cdot \boldsymbol{\omega}$ and $\boldsymbol{\omega} \cdot \mathbf{v}$ are known as the Killing and Klein forms on $\mathfrak{s e ( 3 )}$ and they generate all polynomial invariants (see [70] Sections 4.7 and 6.3). Their ratio

$$
p=\frac{\boldsymbol{\omega} \cdot \mathbf{v}}{\boldsymbol{\omega} \cdot \boldsymbol{\omega}}
$$

is called the pitch of a twist. In the case $\boldsymbol{\omega}=0$, the pitch is defined to be $p=\infty$.

\subsection{Line geometry}

Lines in space and their geometry are central in the study of rigid body kinematics. One of the very earliest theorems in the field of theoretical kinematics is the Mozzi-Chasles Theorem referred to at the start of Section 3.3. According to this theorem, a general rigid body displacement in space is identical to a screw displacment (or simply a screw) in which a rotation is performed 


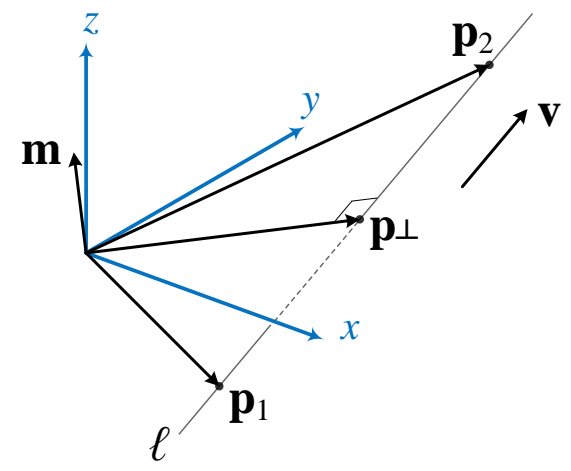

Figure 3.1: Lines in 3-space

about a screw axis followed by a translation along the same axis [16]. The screw axis is considered as a line $\ell$ in $\mathbb{R}^{3}$. We start this section with an overview of lines in space and then we will discuss the more general screw theory.

\subsubsection{Lines in $\mathbb{R}^{3}$}

There are various different representations of a line in 3-dimensional space. In this section, we focus on the parametric form, which we use throughout this thesis, especially in Chapter 4, where we model kinematic pairs using geometric objects such as points and lines.

$$
\mathbf{v}:=\mathbf{p}_{2}-\mathbf{p}_{1}=\left(v_{1}, v_{2}, v_{3}\right)^{T} \in \mathbb{R}^{3}
$$

A point $\mathbf{p}_{\lambda}$ on that line can be determined in the parametric form

$$
\mathbf{p}_{\lambda}:=\mathbf{p}_{1}+\lambda \mathbf{v} \in \ell
$$

where $\lambda \in \mathbb{R}$ is a free parameter. Note that there are infinitely many parametric representations of a given line $\ell$. The point $\mathbf{p}_{1}$ could be replaced by any other point on $\ell$ and if $\mu \in \mathbb{R}, \mu \neq 0$, the vector

$$
\mathbf{v}_{1}=\mu \mathbf{v}
$$


can be used as a direction vector.

Suppose $\ell_{1}: \mathbf{p}_{1}+\lambda_{1} \mathbf{v}_{1}$ and $\ell_{2}: \mathbf{p}_{2}+\lambda_{2} \mathbf{v}_{2}$ are two lines. We will sometimes want to determine if $\ell_{1}=\ell_{2}$. This requires $\mathbf{p}_{2} \in \ell_{1}$ so for some $\lambda_{1} \in \mathbb{R}$, $\mathbf{p}_{2}=\mathbf{p}_{1}+\lambda_{1} \mathbf{v}_{1}$. It also requires $\mathbf{v}_{2}=\mu \mathbf{v}_{1}$ for some $\mu \in \mathbb{R} \neq 0$. Each of these is a vectorial equation in $\mathbb{R}^{3}$ so contains three scalar equations. However, there is a dependency among these three equations due to the presence of the parameters $\lambda_{1}, \mu$. This dependency can be eliminated, reducing the number of scalar equations to two in each case. As a result, there will be four scalar equations in total. So, only four scalar equations are sufficient to specify equality of lines $\ell_{1}, \ell_{2}$ in $\mathbb{R}^{3}$.

\subsubsection{Plücker coordinates}

Alternatively, we can describe a line $\ell$ using a direction vector $\mathbf{v}$ and a $m o-$ ment vector associated to $\ell$. Consider two points $\mathbf{p}_{1}$ and $\mathbf{p}_{2}$ and the line $\ell$ passing through them. Then, there exists a moment vector $\mathbf{m}:=\mathbf{p}_{1} \times \mathbf{v}=$ $\left(m_{1}, m_{2}, m_{3}\right)^{T} \in \mathbb{R}^{3}$ is the moment of $\ell$ about o. Note that, up to scalar multiple, $\mathbf{m}$ is independent of the particular parametrisation used. Replacing $\mathbf{p}_{1}$ by $\mathbf{p}_{1}+\lambda \mathbf{v}$ and $\mathbf{v}$ by $\mu \mathbf{v}(\mu \neq 0)$,

$$
\left(\mathbf{p}_{1}+\lambda \mathbf{v}\right) \times \mu \mathbf{v}=\mu\left(\mathbf{p}_{1} \times \mathbf{v}\right)=\mu \mathbf{m}
$$

Moreover,

$$
\mathbf{p}_{1} \times \mathbf{v}=\mathbf{p}_{1} \times\left(\mathbf{p}_{1}-\mathbf{p}_{2}\right)=\mathbf{p}_{1} \times \mathbf{p}_{\mathbf{2}}
$$

so the coordinates are

$$
m_{1}=a_{2} b_{3}-b_{2} a_{3}, \quad m_{2}=b_{1} a_{3}-a_{1} b_{3}, \quad m_{3}=a_{1} b_{2}-b_{1} a_{2}
$$

Clearly $\mathbf{m}$ is perpendicular to $\mathbf{v}$.

So we have associated two vectors, $\mathbf{v}, \mathbf{m} \in \mathbb{R}^{3}$ with the line $\ell$. However, 
they are not independent since we know $\mathbf{v} \cdot \mathbf{m}=0$. On the other hand, multiplying $\mathbf{v}$ and $\mathbf{m}$ by a non-zero constant $\mu$ gives the same line. This leads us to another description of lines, called Plücker coordinates.

This representation employs six coordinates $\mathbf{u}=\left(p_{01}, p_{02}, p_{03} ; p_{23}, p_{31}, p_{12}\right)$ to specify a line $\ell \in \mathbb{R}^{3}$. Generally speaking, we can assume these coordinates to determine a point in 6 -dimensional hyperspace i.e. $\mathbf{u} \in \mathbb{R}^{6}$. Given a pair of points $\mathbf{p}_{1}=\left(a_{1}, a_{2}, a_{3}\right)^{T}$ and $\mathbf{p}_{2}=\left(b_{1}, b_{2}, b_{3}\right)^{T}$ in $\mathbb{R}^{3}$ set

$$
p_{i j}=a_{i} b_{j}-a_{j} b_{i}, \quad i \neq j=0,1,2,3
$$

where $a_{0}=b_{0}=1$. More generally, Plücker coordinates can be defined on projective 3 -space $\mathbb{P}^{3}$, by taking $\left(a_{0}, a_{1}, a_{2}, a_{3}\right)$ and $\left(b_{0}, b_{1}, b_{2}, b_{3}\right)$ as homogeneous coordinates for points $\mathbf{p}_{1}, \mathbf{p}_{2} \in \mathbb{P}^{3}$. Plücker coordinates are then also homogeneous and define a point in $\mathbb{P}^{5}$. So, the Plücker coordinates of $\ell$ are

$$
\begin{gathered}
p_{01}=b_{1}-a_{1}, \quad p_{02}=b_{2}-a_{2}, \quad p_{03}=b_{3}-a_{3}, \\
p_{23}=a_{2} b_{3}-b_{2} a_{3}, \quad p_{31}=b_{1} a_{3}-a_{1} b_{3}, \quad p_{12}=a_{1} b_{2}-b_{1} a_{2}
\end{gathered}
$$

By comparing (3.24) with (3.20) and (3.23), Plücker coordinates are simply the direction vector and a moment vector of a line, that is, $\mathbf{u}:=(\mathbf{v} ; \mathbf{m}) \in \mathbb{R}^{6}$.

As noted above, a direction vector and moment vector of a line $\ell$ in $\mathbb{R}^{3}$ must be perpendicular i.e. $\mathbf{v} \cdot \mathbf{m}=0$. If we expand this condition in terms of the Plücker coordinates

$$
p_{01} p_{23}+p_{02} p_{31}+p_{03} p_{12}=0
$$

Furthermore, for a line $\ell$ to be defined, we require $\mathbf{v} \neq \mathbf{0}$. Since we may take non-zero multiples of the coordinates, it is common for the direction vector can be normalised i.e. $\mathbf{v} \cdot \mathbf{v}=1$, which gives the additional condition

$$
p_{01}^{2}+p_{02}^{2}+p_{03}^{2}=1
$$


Clearly, equations (3.25) and (3.26) are quadratic equations in $\mathbb{R}^{6}$ each defining a hypersurface. The intersection of these is a 4-dimensional variety which contains all sets of coordinates in $\mathbb{R}^{6}$ that represent lines in $\mathbb{R}^{3}$. We shall refer to this set as the Klein quadric, $\mathcal{K} \subseteq \mathbb{R}^{6}$. Strictly, that term refers to the projective variety in $\mathbb{P}^{5}$ defined by $(3.26)^{2}$. In $\mathbb{R}^{6}$, the points $\mathbf{u},-\mathbf{u} \in \mathcal{K}$ correspond to the same line in $\mathbb{R}^{3}$.

\subsubsection{Screw theory}

In this section, we return to the idea of a screw as a description of finite and instantaneous rigid body displacements and show their connections to lines and their Plücker coordinates.

Following Chasles' Theorem, consider a finite rigid body displacement having a screw axis $\ell$, so rotating about $\ell$ and translating along it. First, let us assume that $\ell$ passes through the origin $\mathbf{o}$ and the direction is given by a unit vector $\hat{\mathbf{v}} \in \mathbb{R}^{3}$. The displacement can be written in the following $(4 \times 4)$ matrix form

$$
\left[\begin{array}{cc}
A_{\phi} & \frac{\phi}{2 \pi} p \hat{\mathbf{v}} \\
0 & 1
\end{array}\right]
$$

where $A_{\phi}$ is the $(3 \times 3)$ rotational matrix representing rotation $\phi$ about the axis $\ell$. Note that $\phi$ is unique only up to addition of multiples of $2 \pi$. In addition, $p$ is a scalar called the pitch of the screw representing the ratio of translation along the line $\phi p$ to the angle of rotation $\phi$.

More generally, suppose the screw axis is free to be anywhere in the space as pictured in Figure 3.1. We want to choose a point on that line. To be specific, we choose the foot $\mathbf{p}_{\perp}$ of the normal from $\mathbf{o}$ to $\ell$. This is the point having the shortest distance from the origin. Note that if $\ell$ passes through $\mathbf{o}$, we choose $\mathbf{p}_{\perp}=\mathbf{0}$. We now change coordinates so that the origin is shifted to $\mathbf{p}_{\perp}$. In other words, it is a rigid displacement which only involves a pure

\footnotetext{
${ }^{2}$ In this setting, $\mathbf{v}=0$ corresponds to a line at infinity in $\mathbb{P}^{3}$.
} 
translation, given in matrix form by

$$
\left[\begin{array}{cc}
I_{3} & \mathbf{p}_{\perp} \\
0 & 1
\end{array}\right]
$$

The impact of this change of coordinates is given by the conjugation operation in Section 3.3.1. Once the axis passes through the origin, we can use (3.27) to represent the rotation about it and so the matrix form is

$$
\left[\begin{array}{cc}
I_{3} & \mathbf{p}_{\perp} \\
0 & 1
\end{array}\right]\left[\begin{array}{cc}
A_{\phi} & \frac{\phi}{2 \pi} p \hat{\mathbf{v}} \\
0 & 1
\end{array}\right]\left[\begin{array}{cc}
I_{3} & -\mathbf{p}_{\perp} \\
0 & 1
\end{array}\right]=\left[\begin{array}{cc}
A_{\phi} & \frac{\phi}{2 \pi} p \hat{\mathbf{v}}+\left(I_{3}-A_{\phi}\right) \mathbf{p}_{\perp} \\
0 & 1
\end{array}\right]
$$

This is a representation of an arbitrary finite screw displacement.

Conversely, suppose any rigid displacement is written in homogeneous form given in (2.10). The rotation $A$ has a fixed axis spanned by a non-zero vector $\boldsymbol{\omega}$, so $A \boldsymbol{\omega}=\boldsymbol{\omega}$. We want to write $\mathbf{t}$ in terms of (3.29) [70]

$$
\mathbf{t}=\frac{\phi}{2 \pi} p \boldsymbol{\omega}+\left(I_{3}-A\right) \mathbf{p}
$$

So we must solve this equation for $p$ and $\mathbf{p}$ in terms of $\phi, \mathbf{t}$ and $\boldsymbol{\omega}$. Taking scalar product with $\boldsymbol{\omega}$ gives

$$
\boldsymbol{\omega} \cdot \mathbf{t}=\frac{\phi}{2 \pi} p \boldsymbol{\omega} \cdot \boldsymbol{\omega}+\boldsymbol{\omega}^{T}\left(I_{3}-A\right) \mathbf{p}
$$

From $A \boldsymbol{\omega}=\boldsymbol{\omega}$, and since $A^{-1}=A^{T}$, we deduce $A^{T} \boldsymbol{\omega}=\boldsymbol{\omega}$ and $\boldsymbol{\omega}^{T} A=\boldsymbol{\omega}^{T}$ so the second term vanishes. Hence

$$
p=\frac{2 \pi}{\phi} \frac{\boldsymbol{\omega} \cdot \mathbf{t}}{\boldsymbol{\omega} \cdot \boldsymbol{\omega}}
$$

This is the finite displacement version of the pitch defined in (3.19) for twists. 
Now rearrange (3.30) as

$$
\left(I_{3}-A\right) \mathbf{p}=\mathbf{t}-\left(\frac{\boldsymbol{\omega} \cdot \mathbf{t}}{\boldsymbol{\omega} \cdot \boldsymbol{\omega}}\right) \boldsymbol{\omega}
$$

The choice of $\mathbf{p}$ ensures the equations are consistent and, since $\left(I_{3}-A\right) \boldsymbol{\omega}=\mathbf{0}$, there is a line of solutions of the form $\mathbf{p}_{0}+\lambda \boldsymbol{\omega}$. For some $\lambda=\lambda_{0}, \mathbf{p}_{\perp}=$ $\mathbf{p}_{0}+\lambda_{0} \boldsymbol{\omega}$ minimises the distance from o. In the special case $A=I_{3}$, there is no privileged eigenvector $\boldsymbol{\omega}$ and $\phi=0$ so (3.30) is inconsistent. This corresponds to pure translation.

The Plücker coordinates for the screw axis are $\left(\boldsymbol{\omega} ; \mathbf{p}_{0} \times \boldsymbol{\omega}\right) \in \mathcal{K}$. Notice that if we add a component $p \boldsymbol{\omega}$ to the second vector, orthogonal to it, we obtain a general 6 -vector $(\boldsymbol{\omega} ; \mathbf{v})=\left(\boldsymbol{\omega} ; \mathbf{p}_{0} \times \boldsymbol{\omega}+p \boldsymbol{\omega}\right)$ that satisfies $\boldsymbol{\omega} \cdot \mathbf{v}=p$. This gives a natural way to extend Plücker coordinates to describe rigid displacements.

\subsection{The exponential map and 1-parameter subgroups}

Thus far, we have observed that associated with a Lie group $G$ is the Lie algebra $\mathfrak{g}$ consisting of the derivatives at $t=0$ of paths $\sigma(t)$ in $G$ with $\sigma(0)=e$. In this section, we introduce a mapping that transforms $X \in \mathfrak{g}$ to an element $g \in G$. This is done by means of the following power series that is called the exponential function. Assume $X$ to be an $(n \times n)$ matrix representing an element of $\mathfrak{g}$, then

$$
\exp X=I+X+\frac{X^{2}}{2 !}+\frac{X^{3}}{3 !}+\ldots
$$

Note that this series converges for any $X$, so $\exp X$ is well defined.

It is a remarkable property of (matrix) Lie groups $G$ and their Lie algebra $\mathfrak{g}$ that for any $X \in \mathfrak{g}, \exp X \in G$, see for example [76]. Thus, exp $: \mathfrak{g} \rightarrow G$. 
If $G$ is not an abelian group, in general, $\exp (X+Y) \neq \exp X \exp Y$. However, if $X Y=Y X$, then $\exp (X+Y)=\exp X \exp Y$. In particular, if $s, t \in \mathbb{R}$, then $\exp [(s+t) X]=\exp (s X) \exp (t X)$. Hence, the function

$$
\sigma_{X}: \mathbb{R} \rightarrow G, \quad \sigma_{X}(t)=\exp (t X)
$$

is a group homomorphism and the image $\{\exp (t X) \mid t \in \mathbb{R}\}$ is a subgroup of $G$, called a 1-parameter subgroup. Note that

$$
\frac{d}{d t}\left[\sigma_{X}(t)\right]_{t=0}=X
$$

so that $\sigma_{x}(t)$ is a path defining $X \in \mathfrak{g}$.

For the rotation and Euclidean groups $S O(3)$ and $S E(3)$, we can use the quaternion and dual quaternion representations to compute the exponential functions, using the homomorphisms $S^{3} \rightarrow S O(3)$ and $\mathbb{D} S^{3} \rightarrow S E(3)$ described in Sections 2.4.2 and 2.4.5.

\subsection{Infinitesimal displacements and twists in the pose space}

In the previous sections, we studied the Lie algebra and the tangent space of the Euclidean group, describing the infinitesimal displacement of an individual rigid body which moves freely in space. We saw that its rigid displacement is expressed by $g \in S E(n)$ and its instantaneous rigid velocity is defined by a twist $\boldsymbol{\xi} \in \mathfrak{s e}(\mathfrak{n})$. The tangent space at a general point $g \in S E(n)$ can be identified with the Lie algebra by a right (or left) pullback as in (3.14).

Following our discussion in Section 2.5, we are going to address the infinitesimal displacement of two bodies connected together by a joint, a 2-body system in which one is fixed, and finally a multi-body system. Let us start with the first case when two rigid bodies $L_{ \pm}$with moving frames $M_{ \pm}$are 
constrained together by a joint $J$. We already saw in (2.44) that the possible displacements of this 2-body system are described by a product of two copies of element of the $S E(n)$. Path derivatives in this product space give tangent space

$$
T_{\left(g_{-}, g_{+}\right)}[S E(n) \times S E(n)] \cong T_{g_{-}} S E(n) \times T_{g_{+}} S E(n)
$$

To describe the instantaneous velocity of one link relative to the other we should work in a tangent space of the homogeneous space $[S E(n) \times$ $S E(n)] / S E(n)$. As noted in Section 2.5, it is sufficient to assume one link fixed and hence to consider just the instantaneous velocity in the second component only. Suppose $\left(\xi_{-}, \xi_{+}\right) \in T_{g_{-}} S E(n) \times T_{g_{+}} S E(n)$ is represented by a path $\left(\sigma_{-}(t), \sigma_{+}(t)\right)$ in $[S E(n) \times S E(n)]$ with $\sigma_{ \pm}(0)=g_{ \pm}$. Then a representative path in the homogeneous space is $(e, \sigma(t))=\left(e, \sigma_{+}(t) \sigma_{-}^{-1}(t)\right)$. Differentiating with respect to $t$ and setting $t=0$ gives $(0, \xi)$, where

$$
\xi=\sigma(0) \in T_{g} S E(n)
$$

and $g=\sigma(0)$. Since elements of the Lie algebra $\mathfrak{s e}(\mathfrak{n})$ have a nice form, it may be useful to now obtain a pullback of $\xi \operatorname{into} \mathfrak{s e}(\mathfrak{n})$. We will use this idea in Section 4.6.

Similarly, relative displacements in M-space given by (2.46) correspond to elements of the product of tangent spaces of $k$ components moving with respect to a fixed base

$$
T_{g^{1}} S E(n) \times T_{g^{2}} S E(n) \times \ldots \times T_{g^{k}} S E(n)
$$

This is a fibre ${ }^{3}$ of the tangent bundle over $\left(g^{1}, \ldots, g^{k}\right) \in[S E(3)]^{k}$, associated to the free motions of the multi-body systems. If we regard all tangent spaces at their associated identity elements and want to represent elements by multi-

\footnotetext{
${ }^{3}$ A fibre of a mapping $f: M \rightarrow N$ is the preimage of an element $y \in N$ [85].
} 
twists, then we can pullback component-wise to $k$ copies of the Lie algebra

$$
\left(\boldsymbol{\xi}_{1}, \boldsymbol{\xi}_{2}, \ldots, \boldsymbol{\xi}_{k}\right) \in[\mathfrak{s e}(\mathfrak{n})]^{k}
$$

Note that the form in (3.38) is associated to the home configuration (i.e. $\left.g_{i}=e, i=1, \ldots, k\right)$. Every twist $\boldsymbol{\xi}_{i}$ may depend on design parameters as well as the current configuration.

In the robot kinematics literature, the matrix whose columns are these twists is sometimes called a Jacobian matrix and its properties, particularly its rank, tells us about the kinematics of the multi-body system. We will explain it with more details in Section 5.1. In this thesis, we use the term Jacobian matrix in its mathematical meaning as the matrix of partial derivatives of a vector-valued multivariable function $f: \mathbb{R}^{m} \rightarrow \mathbb{R}^{n}$. This more general approach, which has the kinematic version as a special case, enables us to make use of results from differential geometry. 


\section{Chapter 4}

\section{Constraints versus freedoms}

So far, we have reviewed preliminaries and mathematical tools that we employ for developing an approach to study kinematics including singularities. This approach involves two main parts. The first part concerning configuration spaces is going to be explained in this chapter whilst the second part concerning singularities will be introduced in the next chapter.

In this chapter, we will discuss how to model the kinematics of a family of manipulators by means of joint constraint equations. This approach will provide us with a set called the design-configuration space (D-space). A family of manipulators consists of a fixed topology, referred to as the architecture of the family, which can be represented combinatorically by a graph in which the links are vertices and edges connect pairs of links that are connected by a joint $[86,87,88]$. Moreover, the edges are annotated by the type of joint, which also determines its degrees of freedom or constraints. So the architecture includes given types of joints. A particular mechanism requires a number of design parameters that can vary within the family. However, topological characteristics are fixed within the family. Now, if a fixed choice of design parameters is assumed, then we will have a subset of D-space, called a configuration space (C-space) determined by a mapping called the kinematic constraint map (KCM). In effect, this is a cross-section of the D-space for 
fixed choices of design parameters.

The C-space of a manipulator with specific choices for design parameters indicates all feasible configurations that the manipulator can occupy. In effect, whether a configuration is feasible depends on the displacements of all components involved in that particular manipulator. This means that we should take into account pose variables corresponding to all links without discrimination. A C-space is generally regular, yet it may occasionally be singular under special circumstances that will occur if certain Grashof-type conditions are satisfied by the design parameters.

The idea of a KCM is to provide a consistent description of the kinematic capabilities of a manipulator, independent of the choice of inputs (actuated joints) and outputs (end-effector poses). While the choice of these is ultimately critical to the application of a manipulator, the $\mathrm{KCM}$ will be able to capture any such choice and from that to determine, among other things, all types of kinematic singularity. The KCM itself is essential for capturing underlying C-space singularities of the whole mechanism.

In this chapter, we are going to perceive a joint from two different perspectives. In the first part, we will specify a joint in terms of constraints that it imposes on two adjacent bodies which are connected together via the joint. In the second part, on the other hand, we will define a joint in terms of freedoms that it permits two adjacent bodies to move relatively with respect to each other. In Section 4.1, we contrast approaches to kinematics based on constraint and on freedom of motion. Then in Section 4.2, we present the associated idea of constraint equations, leading to the concept of the kinematic constraint map defined in Section 4.5. In the following sections we give explicit constraint equations for planar and spatial mechanism joints. 


\subsection{Chebyshev-Grübler-Kutzbach formula}

Suppose two rigid bodies are connected together by a joint $J$ that allows them to displace relative to each other in an ambient space. The joints commonly used in spatial mechanisms are described as Reuleaux's lower pairs, which are determined by the coincidence of surfaces in the rigid bodies. (see, for example, [70]). These give the familiar revolute $(\mathrm{R})$, helical $(\mathrm{H})$ and prismatic (P) joints, the cylindrical (C), spherical (S), and planar (E) joints. All of them are characterised by the fact that the relative freedom between the links is determined by a subgroup in the quotient space. The degrees of freedom (dofs), $\delta$, of the joint is the dimension of the subgroup. In the planar case, the contacting surfaces reduce to curves and we just have $\mathrm{R}$ and $\mathrm{P}$ joints.

Following an approach proposed by Piipponen and Tuomela [61], our primary goal is to establish constraint equations representing a joint. That is, instead of introducing a joint based on the degrees of freedom $\delta$ that it provides the two connected bodies, we model it in terms of $r=p-$ $\delta$ constraints (where $p=\operatorname{dim} S E(n)$, given in (2.9)) that are imposed on relative displacements between the bodies.

Our motivation has arisen from two different forms of the ChebyshevGrübler-Kutzbach (CGK) formula used for determining the global mobility, $\mu$, of a generic multi-body system

$$
\begin{aligned}
\mu & =\sum_{j=1}^{t} \delta_{j}-p(t-k+1) \\
& =p(k-1)-\sum_{j=1}^{t}\left(p-\delta_{j}\right)
\end{aligned}
$$

where $t, \delta_{j}, k$ are the number of joints, the degrees of freedom of joint $J_{j}$, $j=1, \ldots, t$, and the number of links respectively. In particular, the formulae acknowledge a necessary distinction between an approach based on freedoms and one based on constraints. 
The essential point is that although the formulae are equivalent, they describe the global mobility $\mu$ from different perspectives. (4.1a) expresses mobility in terms of joint freedoms, whereas (4.1b) is expressed in terms of joint constraints, so that the forms are complementary. This is because the degrees of freedom and the number of constraints are complementary for a given (either planar or spatial) joint, relative to the dimension of the Euclidean groups. In space where $p=6$, for example, an S-joint has $\delta=3$ so $r=3$. Likewise, for a $\mathrm{C}$-joint $\delta=2$ so $r=4$. In the plane where $p=3$, an R-joint has $\delta=1$ and $r=3-1=2$.

In the following section, we will explain how to attain required constraint equations for a single joint between two rigid bodies.

\subsection{Joint constraint equations}

In a manipulator, suppose that two links $L_{ \pm}$are connected to each other by a joint $J$, thereby constraining their relative displacement. As the first step towards determining the constraint equations associated to the given joint, we allocate a geometric object to it. A particular type of joint can be characterised by a specific object. This object is geometrically equivalent to the joint in regard of freedoms and constraints and is intended to replicate Reuleaux's contact surfaces (for example, see [70] Section 3.6). For instance, an S-joint and a C-joint are modelled by a point $\mathbf{p} \in \mathbb{R}^{3}$ and a directed line $\ell \in \mathbb{R}^{3}$, respectively, in each link. The constraint is defined by the objects coinciding. For example, there are three constraint equations required to determine coincidence of points whereas four constraint equations are needed to specify coincidence of directed lines in space. These correspond to the degrees of freedom and constraints mentioned above.

In the planar case, only two main objects are required, namely a point $\mathbf{p}$ and a directed line $\boldsymbol{\ell}$. On the other hand, in space, in addition to point and directed line, an oriented plane $\boldsymbol{\pi}$ may be needed. Objects are either 
employed individually like the abovementioned examples, or they may join together to form another object ${ }^{1}$. For instance, a point-line is used in order to model a spatial revolute joint, whilst a line-plane is required in order to model a spatial prismatic joint. It is important that the assigned objects are invariant (fixed) with respect to the relative displacements permitted by the joint. We will study all different (planar and spatial) joints and find corresponding constraint equations in Secs. 4.3 and 4.4.

Returning to the general procedure, after identifying the right object which is attached to each rigid body $L_{ \pm}$, for a given joint, body coordinates are assigned to each link via moving frames $M_{ \pm}$. In principle, the frames are arbitrary but it is generally helpful to assign coordinates that specifically align to the joint objects, noting that many links will have more than one joint assigned, so that choices are required. In this case, the positions of the joint objects involve design parameters, e.g. Denavit-Hartenberg (DH) parameters [89]. The design parameters themselves are independent of the choice of frames in the link. We also assign a reference frame $R$ and coordinates to the ambient space.

The choice of frames $M_{ \pm}$(for two connected links) and $R$ determine the displacements of the links as elements $g_{ \pm}=\left(A_{ \pm}, \mathbf{t}_{ \pm}\right) \in S E(n), n=2,3$. The two copies of $S E(n)$, for $M_{ \pm}$, can each be assigned pose variables, which may include quaternions or dual quaternions with additional constraints. We denote the pose variables $\mathbf{u}_{ \pm} \in \mathbb{R}^{p}$ (or potentially $\mathbb{R}^{p+c}$ where additional variables and equations are used).

As the second step in obtaining constraint equations, we need to transform the body coordinates $\mathbf{j}_{ \pm}$for the joint object in $M_{ \pm}$to the reference frame by means of pose variables $\mathbf{u}_{ \pm}$, respectively. For this purpose, (2.6) - called the absolute displacement [78] - is utilised and returns the reference-frame coordinates $\mathbf{J}_{ \pm} \in \mathbb{R}^{n}, n=2,3$ associated to the given joint in the reference

\footnotetext{
${ }^{1}$ These objects are independent and often called oriented flags, i.e. a sequence of oriented linear subspaces [70].
} 
frame $R$

$$
\mathbf{J}_{ \pm}=A_{ \pm} \mathbf{j}_{ \pm}+\mathbf{a}_{ \pm}
$$

The pair of sets of reference-frame coordinates $\mathbf{J}_{ \pm}$are defined in terms of the pair of pose variables $\left(\mathbf{u}_{-}, \mathbf{u}_{+}\right) \in \mathbb{R}^{2 p}$ and may also involve design parameters $\mathbf{s}$ in the relevant space $\mathbb{R}^{d}$. Reference-frame coordinates for the joint object must be equal to each other. In effect, the geometric condition translates into an algebraic condition in reference-frame coordinates

$$
\mathbf{J}_{-} \sim \mathbf{J}_{+}
$$

where $\sim$ denotes coincidence, parallelism, or perpendicularity, the three main geometric conditions that address the dependency of the pair of referenceframe coordinates in the reference frame $R$.

According to the type of joint, (4.3) provides us with a set of $r$ constraint equations $f:=\left(f_{1}, \ldots, f_{r}\right)$ imposed on the pose variables of the moving components and design parameters

$$
f\left(\mathbf{u}_{-}, \mathbf{u}_{+}, \mathbf{s}\right)=\mathbf{0} \in \mathbb{R}^{r}
$$

We call $f$ and $\mathbb{R}^{r}$ a joint constraint mapping and a joint constraint space, respectively. For now, we will treat the design parameters as fixed.

Following our discussion of pose space and relative displacement in Section 2.5, two moving links create a 2-body system in the ambient space. Since (2.44) identifies an element describing the absolute displacement of the whole system, a joint constraint mapping can be thought of as

$$
f: S E(n) \times S E(n) \rightarrow \mathbb{R}^{r}
$$

and the set of displacements of the 2-body system in the ambient space is 
the joint configuration space

$$
\mathcal{C}:=\left\{\left(g_{-}, g_{+}\right) \mid f\left(g_{-}, g_{+}\right)=\mathbf{0}\right\} \subseteq S E(n) \times S E(n)
$$

Given $\left(g_{-}, g_{+}\right) \in \mathcal{C}$ and any $h \in S E(n)$, the fact that the 2-body system is free to move without any relative motion between the links means the following relation holds true

$$
f\left(g_{-}, g_{+}\right)=\mathbf{0} \Leftrightarrow f\left(h \circ g_{-}, h \circ g_{+}\right)=\mathbf{0}
$$

that is, the joint configuration space $\mathcal{C}$ is invariant under the simultaneous Euclidean group action.

As in Section 2.5, let us assume $h=g_{-}^{-1}$ in (4.7), so in effect, $L_{-}$in the 2-body system is fixed and $g_{-}=e \in S E(n)$. Then, we can take the quotient of $\mathcal{C}$ by $S E(n)$ to obtain the relative joint configuration space

$$
\mathcal{C}_{\text {rel }}=\mathcal{C} / S E(n)
$$

The relative displacement within the system is given by

$$
\mathcal{C}_{\text {rel }} \cong\{(e, g) \mid f(e, g)=\mathbf{0}\} \subseteq\{e\} \times S E(n)
$$

where $g=g_{+} \circ g_{-}^{-1}$.

The set of $g \in S E(n)$ such that $(e, g) \in \mathcal{C}_{\text {rel }}$ describes the relative displacements permitted by the joint connecting $L_{-}$and $L_{+}$. It is well known that these sets are subgroups of $S E(n)[46,70]$ or, more generally, cosets, since they only contain the identity if the moving frames $M_{ \pm}$coincide in $L_{ \pm}$ for some feasible configuration. In our approach, it will depend on the joint constraint mapping $f$ that we obtain the same cosets as the relative joint configuration spaces and we will show that is the case later in this chapter. For now, let us assume the above. For the 1-dof joints, namely R, P and $\mathrm{H}$ 
the subgroups of $S E(n)$ with dimension 1 are just the 1-parameter subgroups discussed in Section 3.5. These have the form $\exp (t X)$ where $X \in \mathfrak{s e}(\mathfrak{n})$ and $t \in \mathbb{R}$.

Given the constraint mapping $f: S E(n) \times S E(n) \rightarrow \mathbb{R}^{p-1}$, we can identify $X$ by computing the kernel of its Jacobian at a point $(e, g) \in \mathcal{C}_{\text {rel }}$. This only requires partial derivatives with respect to the second component $g \in S E(n)$, so consider $f_{+}(g)=f(e, g)$ and find

$$
\text { ker } J f_{+}(g) \subseteq T_{g} S E(n)
$$

This should be 1-dimensional and spanned by a vector $\boldsymbol{\xi} \in T_{g} S E(n)$. The right pullback of $\boldsymbol{\xi}$ gives $X \in \mathfrak{s e}(\mathfrak{n})$. Accordingly, the motion of link $L_{+}$, resulting from the freedom of the joint $J$, has the form $\exp (t X) \circ g$.

In the next two sections, we shall determine constraints associated to each type of joint and write down the corresponding joint constraint mapping $f$. Section 4.3 will be about three types of planar joints; namely revolute, prismatic, and double-prismatic, while Section 4.4 will discuss five types spatial joints; namely revolute, prismatic, cylindrical, universal, spherical, and planar. The helical (screw) joint is discussed briefly, but presents significant technical difficulties.

\subsection{Planar kinematic pairs}

As was explained in Section 2.4.3, a planar displacement is described by means of an element of $S E(2)$ given in (2.26). A group element is (globally) parametrised by a 3 -vector given in (2.28). (2.6) is employed to transform given body coordinates in a moving frame $M$ into the reference frame $R$ and obtain corresponding reference-frame coordinates.

There are three types of planar joints; namely revolute $(\mathrm{R})$, prismatic $(\mathrm{P})$, and 2-prismatic (2P). The first two correspond to the 1-parameter subgroups 


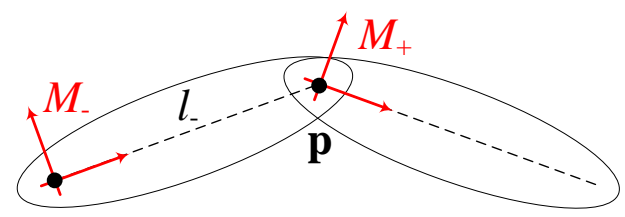

Figure 4.1: Adjacent links constrained at a planar R-joint

while the 2 P-joint corresponds to the translation subgroup $\{I\} \times \mathbb{R}^{2} \subseteq S E(2)$. An R-joint allows two connected components $L_{ \pm}$to relatively rotate about a point whereas a $\mathrm{P}$-joint permits them to translate relatively. The third type can be thought of as two P-joints that are unparalleled so it allows translation in any direction in the plane.

\subsubsection{R-joint constraints}

An R-joint allows two adjacent components to have a relative rotation with respect to each other in the plane, so $\delta=1$. Thus, we can model this joint with a point $\mathbf{p}_{ \pm}$in each link, which should coincide in the reference frame. The next step is to assign moving frames $M_{ \pm}$to the adjacent links $L_{ \pm}$. Although $M_{ \pm}$can be freely attached anywhere on the rigid bodies, a judicious choice is that the associated point $\mathbf{p}$ lies on the positive $x_{-}$-axis in $M_{-}$and is the origin in $M_{+}$. The distance $l_{-}$between the origins in the $M_{ \pm}$is a design parameter - the link length - in the case that there are two successive R-joints.

As shown in Figure 4.1, the body coordinates $\mathbf{p}_{ \pm} \in \mathbb{R}^{2}$ in the preceding and the following moving frames $M_{ \pm}$are

$$
\mathbf{p}_{-}=\left(l_{-}, 0\right)^{T}, \quad \mathbf{p}_{+}=(0,0)^{T}
$$

If the pose variables of $L_{ \pm}$are given by $\left(A_{ \pm}, \mathbf{t}_{ \pm}\right)$, as given in (2.15) and (2.25), respectively, which are used to transform the body coordinates into the ref- 
erence frame $R$, the corresponding pair of reference-frame coordinates are

$$
\mathbf{P}_{ \pm}=A_{ \pm} \mathbf{p}_{ \pm}+\mathbf{t}_{ \pm}
$$

The required condition for establishing an R-joint is the coincidence of reference-frame coordinates in the reference frame $R$. This condition entails the vector equation in terms of the two sets of pose variables

$$
\mathbf{P}_{-}-\mathbf{P}_{+}=\mathbf{0} \Rightarrow A_{-} \mathbf{p}_{-}+\mathbf{t}_{-}-A_{+} \mathbf{p}_{+}-\mathbf{t}_{+}=\mathbf{0} \in \mathbb{R}^{2}
$$

This gives two scalar constraint equations on the pose variables. Note that $\mathbf{p}_{-}$involves $l_{-}$as a design parameter which is therefore embodied in the constraint equations $^{2}$. As a result, the joint constraint mapping $f:=\left(f_{1}, f_{2}\right)$ associated to an R-joint is determined by

$$
\left(t_{x^{-}}, t_{y^{-}}, \theta_{-}, t_{x^{+}}, t_{y^{+}}, \theta_{+}\right) \mapsto\left[l_{-} \cos \theta_{-}+t_{x^{-}}-t_{x^{+}}, l_{-} \sin \theta_{-}+t_{y^{-}}-t_{y^{+}}\right]^{T}
$$

Thus two constraint equations, $f_{1}=f_{2}=0$, are imposed on the pose variables. These fix the translations $\mathbf{t}_{+}$in terms of $\left(A_{-}, \mathbf{t}_{-}\right)$and only allow them to have relative rotation with respect to each other. It is worth noting that other geometric objects could be used to model the relative freedom. In the case of R-joints, circles of equal radius would suffice.

\subsubsection{P-joint constraints}

A P-joint allows two adjacent links to have relative translation in a fixed direction with respect to each other in the plane, so $\delta=1$. This is geometrically modelled by a directed line $\boldsymbol{\ell}_{ \pm}$in each link, whose direction vector indicates the direction of translation. Here, we follow [90] in modelling the constraints. As for the R-joint in Section 4.3.1, assigning two moving frames

\footnotetext{
${ }^{2}$ While the design parameters are features of the coordinate frames $M_{ \pm}$, for a 2-body system they do not have an effect on the relative displacement. They arise when there is more than one joint to consider.
} 


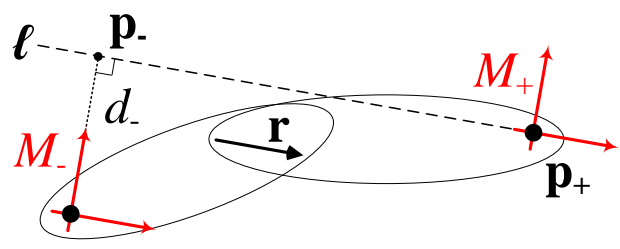

(a)

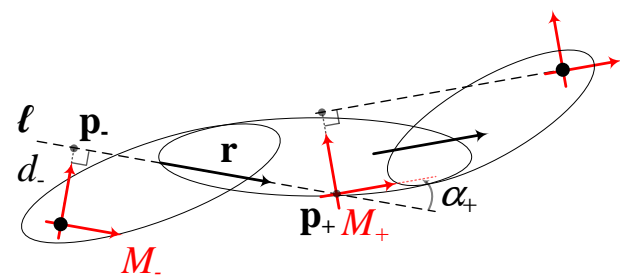

(b)

Figure 4.2: Adjacent links constrained at a planar P-joint: a) RPR-chain and b) RPP-chain

$M_{ \pm}$is the next step. Figure 4.2 shows two cases in which successive joints are $\mathrm{RP}$ or $\mathrm{PP}$.

Suppose a judicious choice has been made for the origin of $M_{-}$, for example located at the centre of a previous $\mathrm{R}$-joint. Choose the $x_{-}$-axis to lie along the direction of the line of the $\mathrm{P}$-joint. Therefore, the $y_{-}$-axis is along a line perpendicular to the $\mathrm{P}$-joint. The perpendicular distance $d_{-} \in \mathbb{R}$ is a design parameter, an offset distance between successive joints. Choose the origin for $M_{+}$to be a point on the line $\boldsymbol{\ell}_{+}$. If $L_{+}$also has an R-joint with a further link, this may be the foot of the perpendicular from the point associated to the R-joint. We may be free to choose the line to pass through that point, in which case it will be the origin. Again, choose the $x_{+}$-direction to be parallel to the direction of the line. On the other hand, if $L_{+}$also has a P-joint, then the origin of $M_{+}$is somewhere on the line of the preceding P-joint (or. may be the intersection of the lines of the two successive P-joints) whereas the $x_{+}$-axis is parallel to the direction of the following $\mathrm{P}$-joint, as shown in Figure $4.2 \mathrm{~b}$. Note that there is a design parameter $\alpha_{+} \in \mathbb{R}$ denoting on the possible angle between successive P-joints.

Suppose the, parametric representation of a line, given in (3.21), is employed to express $\boldsymbol{\ell}$, and so requires a point $\mathbf{p} \in \mathbb{R}^{2}$ on it and a direction vector $\mathbf{r} \in \mathbb{R}^{2}$, together with a parameter $\lambda$. Note that, as for the $\mathrm{R}$-joint, there are alternative choices of object. In the case of a P-joint, any other line parallel to the chosen one can be used. However, the constraint equations 
between the successive objects must model the relative displacement.

Identifying the lines provides constraint equations for a P-joint. As visualised in Figure $4.2 \mathrm{a}$, body coordinates of $\mathbf{p}_{ \pm}$and $\mathbf{r}_{ \pm}$in the preceding and the following moving frames $M_{ \pm}$are

$$
\begin{array}{ll}
\mathbf{p}_{-}=\left(d_{-}, 0\right)^{T}, & \mathbf{p}_{+}=(0,0)^{T} \\
\mathbf{r}_{-}=(1,0)^{T}, & \mathbf{r}_{+}=(1,0)^{T}
\end{array}
$$

The choice of unit vectors for $\mathbf{r}_{ \pm}$means that the parameters for $\boldsymbol{\ell}_{ \pm}$have the same scale (distance measure), so can be identified, $\lambda_{-}=\lambda_{+}=\lambda$, say. Pose variables $\left(A_{ \pm}, \mathbf{t}_{ \pm}\right)$for the links are employed to identify reference-frame coordinates of points of the given directed lines in the reference frame $R$

$$
\left(A_{ \pm}, \mathbf{t}_{ \pm}\right)\left(\mathbf{p}_{ \pm}+\lambda \mathbf{r}_{ \pm}\right)=\mathbf{P}_{ \pm}+\lambda \mathbf{R}_{ \pm}
$$

where $\mathbf{P}_{ \pm}$is given by (4.11) and $\mathbf{R}_{ \pm}=A_{ \pm} \mathbf{r}_{ \pm}$.

There are two required conditions imposed on reference-frame coordinates for modelling a planar P-joint. The two direction vectors must be parallel, and must also be parallel to the vector connecting the two points. These geometric conditions provide us with two scalar constraint equations in terms of the sets of pose variables

$$
\begin{array}{r}
\left(\mathbf{R}_{-} \times \mathbf{R}_{+}\right)_{z}=0 \\
\left(\left(\mathbf{P}_{-}-\mathbf{P}_{+}\right) \times \mathbf{R}_{+}\right)_{z}=0
\end{array}
$$

Here, $\times$ denotes the 3 -dimensional cross product and we treat 2 -vectors as 3 -vectors by appending a third coordinate $z=0$. The subscript $z$ is a reminder that each equation is essentially scalar, the other components of the cross product being zero.

Although $d_{-} \in \mathbb{R}$ as a design parameter appears in the constraint equations, it does not affect the relative displacements of the links. Using the 
above choice of moving frames $M_{ \pm}$, the joint constraint mapping $f:=\left(f_{1}, f_{2}\right)$ associated to a $\mathrm{P}$-joint is

$$
\begin{aligned}
\left(t_{x^{-}}, t_{y^{-}}, \theta_{-}, t_{x^{+}}, t_{y^{+}}, \theta_{+}\right) \mapsto & {\left[-\sin \left(\theta_{+}-\theta_{-}\right), d_{-}-\left(t_{x^{-}}-t_{x^{+}}\right) \sin \theta_{+}\right.} \\
& \left.+\left(t_{y^{-}}-t_{y^{+}}\right) \cos \theta_{+}\right]^{T}
\end{aligned}
$$

The constraint equations given by setting the right-hand side of (4.16) to zero are imposed on free rotation and a translation of the two components and only allow them to have a relative translation with respect to each other. That is, given $\left(A_{-}, \mathbf{t}_{-}\right)$the rotation $\theta_{+}$is given by the first equation, while the second restricts $\mathbf{t}_{+}$to lie on a line.

\subsubsection{P-joint constraints}

Although a 2P-joint can be thought of as two separated 1-dof P-joints, let us consider it as an individual joint whose $\delta=2$. Two adjacent links are only free to translate along the $x, y$-axes, so the only constraint is imposed on their orientation. Thus, the geometric object will be a vector whose body coordinates are given by any non-zero vector e.g. $\mathbf{r}_{ \pm}=(1,1)^{T}$. By transforming these coordinates to the reference frame $R$ via pose variables, reference-frame coordinates $\mathbf{R}_{ \pm}$are obtained.

The geometric condition required for a $2 \mathrm{P}$-joint is that direction vectors must always remain parallel in the ambient space i.e. $\left(\mathbf{R}_{-} \times \mathbf{R}_{+}\right)_{z}=0$. Accordingly, a joint constraint mapping associated to this joint only involves a single constraint equation

$$
\sin \left(\theta_{+}-\theta_{-}\right)=0
$$

which is imposed on free displacement of two adjacent links and so allows them to have arbitrary relative translations with respect to each other. 


\subsection{Spatial kinematic pairs}

As discussed in Section 2.4.3, a spatial displacement in $\mathbb{R}^{3}$ has six degrees of freedom usually described as three rotations about and three translations along orthogonal axes. This displacement is an element of the Euclidean group $S E(3)$. If a dual quaternion is utilised to represent this element, then it is locally represented by an 8-vector given in (2.34) whose entries meet the conditions given in (2.38). We employ (2.6) in order to transform given body coordinates in a moving frame $M$ into the reference frame $R$ and obtain corresponding reference-frame coordinates.

There are five lower types of spatial joints, namely revolute (R), prismatic $(\mathrm{P})$, helical $(\mathrm{H})$, cylindrical $(\mathrm{C})$, spherical $(\mathrm{S})$, and planar $(\mathrm{E})$. It is also common to include the universal (U) joint which is an arrangement of two R-joints with intersecting axes. However, it is not a lower pair and the relative displacement it allows, does not form a subgroup of $S E(3)$. The first three types provide adjacent components with one degree of freedom. R-joints and P-joints allow connected components $L_{ \pm}$to be relatively rotated and translated, respectively. An $\mathrm{H}$-joint permits two links to have a relative screw motion as described in Section 3.4.3. A screw motion is regarded as a composition of a rotation about an axis followed by a translation along that axis. Note that in continuous motion, this rotation cannot be made independently from the translation.

The other four types, on the other hand, provide connected links with more relative freedoms i.e. $\delta \geq 2$

- C-joint: $\delta=2$, a translation along and an independent rotation about an axis

- S-joint: $\delta=3$, three independent rotations about three orthogonal axes through a point

- E-joint: $\delta=3$, two translations along axes in a plane and a rotation 
about an axis perpendicular to that plane

- U-joint: $\delta=2$, two independent rotations about two perpendicular axes

Note that an E-joint permits relative planar displacements. In effect, the relative displacements generated by each of these four spatial joints are in the form of combination of either translations, rotations, or both. Those joints can be considered as a combination of some P-joints and R-joints.

\subsubsection{R-joint constraints}

A spatial R-joint provides connected components with a relative rotation, so $\delta=1$. Hence, there are five constraints required in the 3 -dimensional space. It can be modelled by a directed line $\boldsymbol{\ell}$ with a point on it. The procedure is started by assigning two moving frames $M_{ \pm}$to the adjacent moving components $L_{ \pm}$. This can be done following the procedure of modified Denavit-Hartenberg (DH) convention [91] provided successive joints are R-joints. Helpful guidelines for placing a frame $M$ are as follows

- $z$-axis along the direction of the joint axis

- origin at the foot of common normal between successive axes

- $x$-axis is along the common normal between successive axes

- $y$-axis chosen to form a right-handed basis of coordinates

In general, we may assume that a line determining the $z$-axis has been assigned for the frame $M_{-}$and we construct the common normal from this line to the line $\boldsymbol{\ell}$ representing the $\mathrm{R}$-joint axis in $L_{-}$. The foot of this normal is the origin of the $M_{-}$frame and the $x_{-}$-axis is directed along it. The line $\boldsymbol{\ell}$ in $L_{+}$determines the $z_{+}$-axis and the origin and the $x_{+}$-axis are then chosen. The choice may depend on any additional joint connecting $L_{+}$. As in the DH 


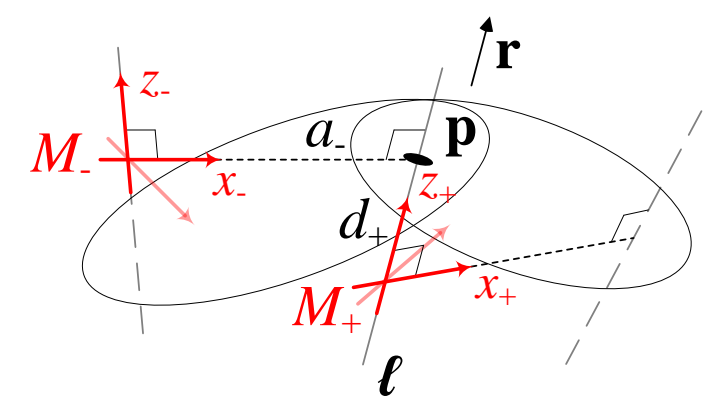

Figure 4.3: Adjacent links constrained at a spatial R-joint

procedure, a number of design parameters determine the relations between successive coordinates in frames $M_{ \pm}$. These are

- the link length $a_{-}$which is the distance between the feet of the common normal between $z_{ \pm}$-axes in $M_{ \pm}$

- the joint angle $0 \leq \psi_{-} \leq \pi$ which is the angle between the directions of the $z_{ \pm}$-axes in $M_{ \pm}$

- the offset $d_{+}$which is the distance along the $z_{+}$-axis between the foot of the common normal in the $L_{-}$and the origin in $M_{+}$

Having assigned coordinate frames, the point-line modelling the joint can be given by parametric representation of a line given in $(3.21), \mathbf{p}+\lambda \mathbf{r}$, in which the point $\mathbf{p} \in \mathbb{R}^{3}$ indicates a chosen base point and the direction vector $\mathbf{r} \in \mathbb{R}^{3}$ gives the direction of the axis about which the rotation is performed. As illustrated in Figure 4.3, we choose the foot of the common normal between the $z_{ \pm}$-axes on the line, so that body coordinates associated to the point and the direction vector in the preceding and the following moving frames $M_{ \pm}$are

$$
\begin{array}{ll}
\mathbf{p}_{-}=\left(a_{-}, 0,0\right)^{T}, & \mathbf{p}_{+}=\left(0,0, d_{+}\right)^{T} \\
\mathbf{r}_{-}=\left(0,-\sin \psi_{-}, \cos \psi_{-}\right)^{T}, & \mathbf{r}_{+}=(0,0,1)^{T}
\end{array}
$$

Note that $\mathbf{r}_{ \pm}$are chosen to be unit vectors so that the lines' parameters match. Pose variables $\left(A_{ \pm}, \mathbf{t}_{ \pm}\right)$are used to determine reference-frame coor- 
dinates in the reference frame $R$

$$
\left(A_{ \pm}, \mathbf{t}_{ \pm}\right)\left(\mathbf{p}_{ \pm}+\lambda \mathbf{r}_{ \pm}\right)=\mathbf{P}_{ \pm}+\lambda \mathbf{R}_{ \pm}
$$

where $\mathbf{P}_{ \pm}=A_{ \pm} \mathbf{p}_{ \pm}+\mathbf{t}_{ \pm}$while $\mathbf{R}_{ \pm}=A_{ \pm} \mathbf{r}_{ \pm}$.

The lines must coincide in the reference frame so two geometric conditions must be satisfied by their reference-frame coordinates. The first condition is that the two points must coincide, while the second condition requires the direction vectors remain equal in the reference frame $R$

$$
\mathbf{P}_{-}=\mathbf{P}_{+}, \quad \mathbf{R}_{-}=\mathbf{R}_{+}
$$

In effect, the second equation amounts to two constraints since $\mathbf{R}_{ \pm}$are unit vectors, so points on a 2-sphere. Nevertheless, we would like to be able to replace this single vector equation in $\mathbb{R}^{3}$ by just two equations. It is worth noting that, since $\mathbf{R}_{ \pm}$are unit vectors, the condition is equivalent to $\mathbf{R}_{-} \cdot \mathbf{R}_{+}=1$ which is just a single equation. However, as we shall see in Section 5.1, the solutions, $\mathbf{R}_{-}-\mathbf{R}_{+}=\mathbf{0}$, are not regular points of the associated function $\mathbb{R}^{3} \rightarrow \mathbb{R}$ and we cannot use this condition if we later wish to determine singularities. An alternative approach is to observe that $\mathbf{R}_{-} \| \mathbf{R}_{+}$and so $\mathbf{R}_{-} \times \lambda \mathbf{R}_{+}=\mathbf{0}$ for some (non-zero) $\lambda \in \mathbb{R}$. Eliminating $\lambda$ reduces this condition to two scalar equations in the pose variables. It, however, introduces spurious solutions corresponding to $\mathbf{R}_{-}=-\mathbf{R}_{+}$. We will address this issue later.

In practice, we use dual quaternions $\breve{\mathbf{q}}_{ \pm}:=\mathbf{q}_{p^{ \pm}}+\varepsilon \mathbf{q}_{d^{ \pm}}$as pose variables. We know that each dual quaternion has to fulfil two extra conditions given in (2.38a) and (2.38b) in order to represent an element of $S E(3)$. In dual quaternions, from (2.39) in Section 2.4.5, the constraint conditions (4.19) are

$$
\left(\mathbf{q}_{p^{+}} \mathbf{p}_{+} \mathbf{q}_{p^{+}}^{*}+\mathbf{t}_{+}\right)-\left(\mathbf{q}_{p^{-}} \mathbf{p}_{-} \mathbf{q}_{p^{-}}^{*}+\mathbf{t}_{-}\right)=0
$$




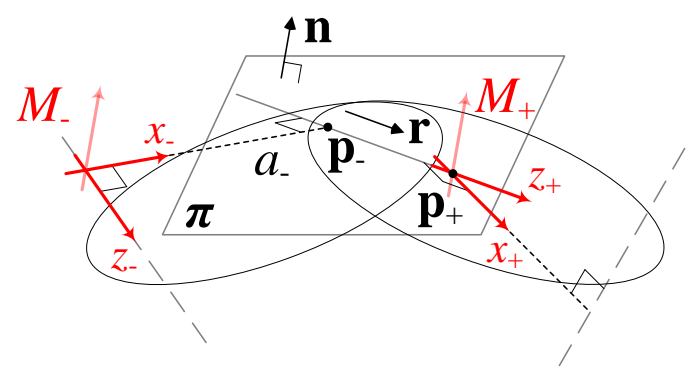

Figure 4.4: Adjacent links constrained at a spatial P-joint

$$
\mathbf{q}_{p^{+}} \mathbf{r}_{+} \mathbf{q}_{p^{+}}^{*}-\mathbf{q}_{p^{-}} \mathbf{r}_{-} \mathbf{q}_{p^{-}}^{*}=0
$$

where $\mathbf{t}_{ \pm}=\mathbf{q}_{d^{ \pm}} \mathbf{q}_{p^{ \pm}}^{*}-\mathbf{q}_{p^{ \pm}} \mathbf{q}_{d^{ \pm}}^{*}$. As above, we can reduce equation (4.20b) to two scalar equations by eliminating a parameter. As a result, there are in total nine constraint equations, as in (A.1), that specify a joint constraint mapping $f:=\left(f_{1}, \ldots, f_{9}\right)$ associated to a spatial R-joint.

\subsubsection{P-joint constraints}

A spatial P-joint connecting two links allows them to have a relative translation, so $\delta=1$. Thus, there are five constraints required in the 3-dimensional space. An equivalent geometric object that characterises a P-joint is an oriented plane $\boldsymbol{\pi}$ defined by a point and a directed line. Note that this object is described by three separate objects - a point, a directed line, and an oriented plane - for obtaining constraint equations. Moving frames $M_{ \pm}$are assigned to the links $L_{ \pm}$according to the guidelines provided in Section 4.4.1. As illustrated in Figure 4.4, $a_{-}, \psi_{-}$are design parameters determining the relations between successive coordinates in frames $M_{ \pm}$.

An oriented plane is specified by a point $\mathbf{p}$ on it and its normal vector $\mathbf{n}$

$$
\boldsymbol{\pi}:=\mathbf{n} \cdot(\mathbf{x}-\mathbf{p})=0
$$

where $\mathbf{x}$ is any point on the plane. There is a line in the plane, $\mathbf{p}+\lambda \mathbf{r}$, whose direction vector, $\mathbf{r} \in \mathbb{R}^{3}$, is parallel to the plane. Note that $\mathbf{n}$ is perpendicular 
to $\mathbf{r}$, which represents the direction along which the translation is carried out. The point $\mathbf{p} \in \mathbb{R}^{3}$ can be chosen anywhere on the directed line.

As illustrated in Figure 4.4, consistent with guidelines, body coordinates associated to the point, direction and normal vectors in the preceding and the following moving frames $M_{ \pm}$are

$$
\begin{array}{ll}
\mathbf{p}_{-}=\left(a_{-}, 0,0\right)^{T}, & \mathbf{p}_{+}=(0,0,0)^{T} \\
\mathbf{r}_{-}=\left(0,-\sin \psi_{-}, \cos \psi_{-}\right)^{T}, & \mathbf{r}_{+}=(0,0,1)^{T} \\
\mathbf{n}_{-}=\left(0, \cos \psi_{-}, \sin \psi_{-}\right)^{T}, & \mathbf{n}_{+}=(0,1,0)^{T}
\end{array}
$$

Note that $\mathbf{r}_{ \pm}, \mathbf{n}_{ \pm}$are chosen to be unit vectors. Pose variables $\left(A_{ \pm}, \mathbf{t}_{ \pm}\right)$are employed to determine reference-frame coordinates in the reference frame $R$

$$
\mathbf{P}_{ \pm}=A_{ \pm} \mathbf{p}_{ \pm}+\mathbf{t}_{ \pm}, \quad \mathbf{R}_{ \pm}=A_{ \pm} \mathbf{r}_{ \pm}, \quad \mathbf{N}_{ \pm}=A_{ \pm} \mathbf{n}_{ \pm}
$$

Note that since $A_{ \pm}$are orthogonal, $\mathbf{R}_{ \pm} \cdot \mathbf{N}_{ \pm}=0$.

The constraints imposed by the P-joint are modelled by requiring the oriented plane and directed line in each of the joined links to coincide in the reference frame. This gives three geometric conditions that must be satisfied. The direction vectors $\mathbf{R}_{ \pm}$and normal vectors $\mathbf{N}_{ \pm}$must be pairwise equal in the ambient space. The displacement vector between the two points needs to be parallel to the direction vectors of the lines in the reference frame $R$

$$
\mathbf{R}_{-}=\mathbf{R}_{+}, \quad \mathbf{N}_{-}=\mathbf{N}_{+}, \quad \mathbf{P}_{-}-\mathbf{P}_{+}=\lambda \mathbf{R}_{+}
$$

The first two conditions prevent any relative rotation between the links and amount to three independent constraints. In fact, they can be more simply expressed in the form

$$
A_{+} A_{-}^{-1}=\text { constant }
$$

where the 'constant' is the rotation aligning the reference frames $M_{ \pm}$in 
any given configuration. The third condition restricts two translations and reduces to two constraint equations. A dual quaternion representation of a spatial displacement satisfies two extra conditions. Consequently, there are totally nine constraint equations, as in (A.2), that specify a joint constraint mapping $f:=\left(f_{1}, \ldots, f_{9}\right)$ associated to a spatial P-joint.

\subsubsection{H-joint constraints}

The H-joint is harder to model by constraints than the other joints because the screw motion involves both rotation about, and translation along, an axis that are related to each other. The $\mathrm{H}$-joint is not used in most manipulators so we do not picture it in much detail. As noted in [92], the constraint variety is not an algebraic variety.

\subsubsection{C-joint constraints}

A C-joint permits adjacent components to have both relative rotation about and translation along an axis, so $\delta=2$. It is then equivalent to an R-joint and a P-joint, independently. However, we can avoid imposing two sets of constraint equations by use of an appropriate geometric object for the joint. The required object is a directed line $\mathbf{p}+\lambda \mathbf{r}$ with a point $\mathbf{p}$ on it. As usual, we choose $\mathbf{r}$ to be a unit direction vector.

Moving frames $M_{ \pm}$are placed based on the guidelines given in Section 4.4.1. Thus, body coordinates of points and the direction vectors in the preceding and the following moving frames $M_{ \pm}$are

$$
\begin{array}{ll}
\mathbf{p}_{-}=\left(a_{-}, 0,0\right)^{T}, & \mathbf{p}_{+}=(0,0,0)^{T}, \\
\mathbf{r}_{-}=\left(0,-\sin \psi_{-}, \cos \psi_{-}\right)^{T}, & \mathbf{r}_{+}=(0,0,1)^{T}
\end{array}
$$

where $a_{-}, \psi_{-}$are design parameters. There are two conditions satisfied by reference-frame coordinates for establishing a C-joint. In terms of pose vari- 


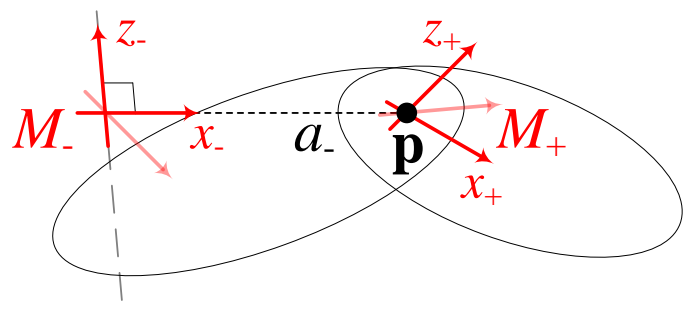

Figure 4.5: Adjacent links constrained at a S-joint

ables $\left(A_{ \pm}, \mathbf{t}_{ \pm}\right)$, if we set $\mathbf{P}_{ \pm}=A_{ \pm} \mathbf{p}_{ \pm}+\mathbf{t}_{ \pm}$and $\mathbf{R}_{ \pm}=A_{ \pm} \mathbf{r}_{ \pm}$, then we require

$$
\mathbf{R}_{-}=\mathbf{R}_{+}, \quad \mathbf{P}_{-}-\mathbf{P}_{+}=\lambda \mathbf{R}_{+}
$$

By eliminating two scalars, only four constraint equations are left for a C-joint in terms of pose variables. Using dual quaternions for the pose variables and including the four extra constraints, there are totally eight constraint equations, as in (A.3), that specify a joint constraint mapping $f:=\left(f_{1}, \ldots, f_{8}\right)$ associated to a C-joint.

\subsubsection{S-joint constraints}

An S-joint is equivalent to three independent $\mathrm{R}$-joints through a fixed point and provides three rotational degrees of freedom between adjacent components, so $\delta=3$. The obvious object to model the constraints of an S-joint is therefore the centre point $\mathbf{p}$. Note that origin of $M_{+}$is at the centre point, as illustrated in Figure 4.5. Denote the distance between the origins of $M_{ \pm}$ by $a_{-}$which is a design parameter. Then, body coordinates corresponding to the point in the preceding and the following moving frames $M_{ \pm}$are

$$
\mathbf{p}_{-}=\left(a_{-}, 0,0\right)^{T}, \quad \mathbf{p}_{+}=(0,0,0)^{T}
$$

Pose variables $\left(A_{ \pm}, \mathbf{t}_{ \pm}\right)$are employed to transform the given point coordinates to the reference frame $R$. This transformation yields reference-frame 
coordinates associated to the S-joint in the ambient space

$$
\mathbf{P}_{ \pm}=A_{ \pm} \mathbf{p}_{ \pm}+\mathbf{t}_{ \pm}
$$

Coincidence is the only geometric condition to be satisfied by reference-frame coordinates given in (4.23) for initiating an S-joint

$$
\mathbf{P}_{-}=\mathbf{P}_{+}
$$

This condition gives three scalar equations in terms of pose variables. In addition to these equations, for the dual quaternion representation, there are four extra constraints that must be fulfilled. As a result, there are totally seven constraint equations, as in (A.5), that specify a joint constraint mapping $f:=\left(f_{1}, \ldots, f_{7}\right)$ associated to an S-joint.

\subsubsection{E-joint constraints}

An E-joint allows adjacent links to have two relative translations in a plane and a relative rotation about an axis perpendicular to the plane, so $\delta=3$. This is therefore planar motion with respect to that plane. A geometric object which characterises an E-joint is an oriented plane $\boldsymbol{\pi}$ on which a point $\mathbf{p}$ lies. An oriented plane is specified by its normal vector $\mathbf{n}$.

The procedure of placing moving frames $M_{ \pm}$follows what is suggested in Section 4.4.1. Except, the origin of $M_{-}$is where the plane meets the joint axis while the origin of $M_{+}$is somewhere on the plane and $z_{+}$is perpendicular to it. Denote the angle between $z_{ \pm}$by $\psi_{-}$which is a design parameter. As illustrated in Figure 4.6, body coordinates associated to the point and the normal vectors in the preceding and the following moving frames $M_{ \pm}$are

$$
\begin{array}{ll}
\mathbf{p}_{-}=(0,0,0)^{T}, & \mathbf{p}_{+}=(0,0,0)^{T} \\
\mathbf{n}_{-}=\left(0,-\sin \psi_{-}, \cos \psi_{-}\right)^{T}, & \mathbf{n}_{+}=(0,0,1)^{T}
\end{array}
$$




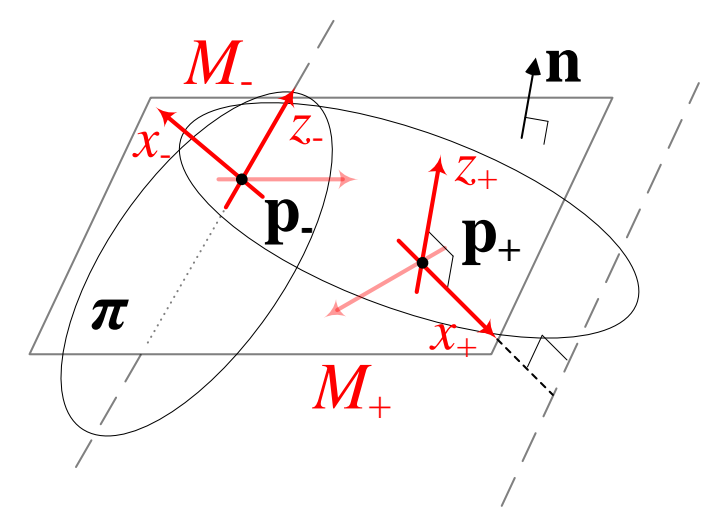

Figure 4.6: Adjacent links constrained at an E-joint

Pose variables $\left(A_{ \pm}, \mathbf{t}_{ \pm}\right)$are utilised to determine reference-frame coordinates of points and normal vectors in the reference frame $R$

$$
\mathbf{P}_{ \pm}=A_{ \pm} \mathbf{p}_{ \pm}+\mathbf{t}_{ \pm}, \quad \mathbf{N}_{ \pm}=A_{ \pm} \mathbf{n}_{ \pm}
$$

For specifying an E-joint, two conditions must be met by reference-frame coordinates: normal vectors must be equal while the displacement vector between two points should be perpendicular to the normal vectors

$$
\mathbf{N}_{-}=\mathbf{N}_{+}, \quad\left(\mathbf{P}_{-}-\mathbf{P}_{+}\right) \perp \mathbf{N}_{+}
$$

Since normal vectors are chosen to be unit vectors, so the first condition implies only two constraint equations.

Four extra constraints are required if the dual quaternion representation is used, so there are totally seven constraint equations, as in (A.4), that specify a joint constraint mapping $f:=\left(f_{1}, \ldots, f_{7}\right)$ associated to a spatial E-joint. 


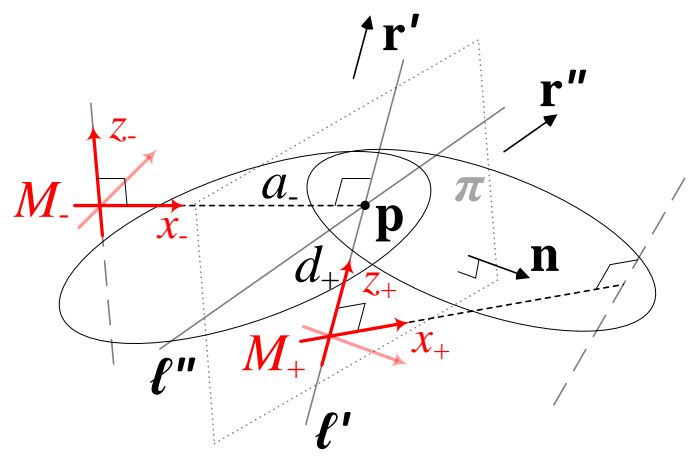

Figure 4.7: Adjacent links constrained at a U-joint

\subsubsection{U-joint constraints}

A U-joint consists of two R-joints with intersecting axes, so it provides two relative rotations between adjacent components and $\delta=2$. Hence, a geometric object associated to a $\mathrm{U}$-joint is two directed lines $\ell^{\prime}, \ell^{\prime \prime}$ intersecting at a point p. Although these lines can be assumed to be in any directions, a special case occurs when they are orthogonal (say along $x, z$-axes of the Cartesian coordinate system), then the normal vector will be along the $y$-axis.

The procedure of assigning moving frames $M_{ \pm}$to the adjacent components $L_{ \pm}$is similar to what is explained in Section 4.4.1. As illustrated in Figure 4.7, it is supposed that $\ell^{\prime}$ represents the joint axis so $\mathbf{r}^{\prime}$ is along the $z_{+}$-axis. Denote the angle between the two directed lines by $\varphi_{+}$and the angle between $z_{+}$and $z_{-}$by $\psi_{-}$which are design parameters along with $a_{-}, d_{+}$. Hence, body coordinates associated to the point, direction vectors and the normal vector in the preceding and the following moving frames $M_{ \pm}$are

$$
\begin{array}{ll}
\mathbf{p}_{+}=\left(0,0, d_{+}\right)^{T}, & \mathbf{p}_{-}=\left(a_{-}, 0,0\right)^{T} \\
\mathbf{r}_{+}^{\prime}=(0,0,1)^{T}, & \mathbf{r}_{-}^{\prime}=\left(0,-\sin \psi_{-}, \cos \psi_{-}\right)^{T} \\
\mathbf{r}_{+}^{\prime \prime}=\left(\cos \varphi_{+}, \sin \varphi_{+}, 0\right)^{T}, & \mathbf{r}_{-}^{\prime \prime}=\left(0,-\sin \left(\psi_{-}+\varphi_{+}\right), \cos \left(\psi_{-}+\varphi_{+}\right)\right)^{T} \\
\mathbf{n}_{+}=\left(-\sin \varphi_{+}, \cos \varphi_{+}, 0\right)^{T}, & \mathbf{n}_{-}=\left(\sin \varphi_{+}, 0,0\right)^{T}
\end{array}
$$

where $d_{+}, a_{-}, \varphi_{+}, \psi_{-}$are design parameters. Pose variables $\left(A_{ \pm}, \mathbf{t}_{ \pm}\right)$are used to transform given body coordinates to the reference frame $R$. This transformation yields reference-frame coordinates corresponding to the U-joint in 
the ambient space

$$
\left(A_{ \pm}, \mathbf{t}_{ \pm}\right)\left(\mathbf{p}_{ \pm}+\tau \mathbf{n}_{ \pm}\right)=\mathbf{P}_{ \pm}+\tau \mathbf{N}_{ \pm}, \quad \mathbf{R}_{ \pm}^{\prime}=A_{ \pm} \mathbf{r}_{ \pm}^{\prime}
$$

where $\mathbf{P}_{ \pm}=A_{ \pm} \mathbf{p}_{ \pm}+\mathbf{t}_{ \pm}$and $\mathbf{N}_{ \pm}=A_{ \pm} \mathbf{n}_{ \pm}$.

To establish a U-joint, two geometric conditions ought to be fulfilled by reference-frame coordinates. The first condition emphasises coincidence of two points while the second one stresses perpendicularity of a normal vector with either of the direction vectors in the reference frame $R$

$$
\mathbf{P}_{-}=\mathbf{P}_{+}, \quad \mathbf{R}_{-}^{\prime} \perp \mathbf{N}_{+}
$$

These two conditions provides us with four constraint equations for a U-joint in terms of a pair of pose variables in the reference frame $R$. In addition, there are four extra constraints that must be fulfilled by dual quaternions corresponding to two moving components. As a result, there are totally eight constraint equations, as in (A.6), that specify a joint constraint mapping $f:=\left(f_{1}, \ldots, f_{8}\right)$ associated to a U-joint.

In Sections 4.3 and 4.4, we have presented geometric objects whose invariance constrains the relative displacement between links according to the way in which they are joined. The invariance can be expressed in terms of joint constraint equations. The number of joint's constraints is the complement of the joint's degrees of freedom. Further, we have shown how, in general, coordinate frames can be chosen so that the equations take a relatively simple form. In the planar case, pose variables are simply $\left(\theta, t_{x}, t_{y}\right) \in \mathbb{R}^{3}$. In the spatial case, dual quaternions can be used. However, this requires the addition of further equations to ensure that a dual quaternion represents a spatial rigid displacement.

In the next section, we generalise the setting to multi-body (rather than 2-body) systems. 


\subsection{Kinematic constraint mapping}

Consider a family of manipulators, with $k+1$ rigid bodies $L_{i}, i=0, \ldots, k$, in which one component (e.g. $\left.L_{0}\right)$ is fixed. The family is assumed to have a fixed architecture - that is, its kinematic graph is fixed - but has a number of design parameters that may vary. Pose variables of the $k$ moving components generate the pose space $\mathcal{M}:=S E(n)^{k}$ as discussed in Section 2.5. Further, suppose this system involves $t$ joints $J_{j}, j=1, \ldots, t$ pairwise connecting links $L_{j_{-}}, L_{j_{+}}$where $j_{-}, j_{+} \in 1, \ldots, t, j_{-} \neq j_{+}$. For a parallel manipulator, where a link is connected to more than one other, the choice of moving frame can only be made with respect to one of the joints and there will be additional design parameters relating to the second or subsequent joints. Each joint, $J_{j}$, imposes $r_{j}$ constraint equations on the pose variables of the connected links.

There are $t$ joint constraint equations like the one given in (4.4)

$$
f_{j}\left(\mathbf{u}_{j_{-}}, \mathbf{u}_{j_{+}}, \mathbf{s}_{j}\right)=\mathbf{0} \in \mathbb{R}^{r_{j}}, j=1, \ldots, t
$$

where $r_{j}$ is the number of constraints imposed by the joint $J_{j}$ and $\mathbf{s}_{j}$ is a vector of design parameters associated with the joint. All constraint mappings together form a vector-valued mapping called the design-kinematic constraint mapping (D-KCM)

$$
\hat{F}:=\left(f_{1}, \ldots, f_{s}\right): \mathcal{M} \times \mathbb{R}^{d} \rightarrow \mathbb{R}^{s}
$$

where $s=\sum_{j=1}^{t} r_{j}$ is the total number of constraint equations and $\mathbb{R}^{d}$ is the design space whose dimension $d$ is the total number of design parameters corresponding to a manipulator's architecture.

The D-KCM consists of constraint equations imposed by all joints on free displacements of moving components dependent on the design parameters. Varying them may alter the topology of the configuration space. As a result, 
$\hat{F}^{-1}\left(\mathbf{0}_{r}\right)$-with the assumption that $\mathbf{0}_{r}$ is a regular value of $\hat{F}$ - defines a manifold called design-configuration space (D-space) associated to a family of mechanisms

$$
\mathcal{D}:=\left\{\left(g_{1}, \ldots, g_{k}, \mathbf{s}\right) \mid \hat{F}\left(g_{1}, \ldots, g_{k}, \mathbf{s}\right)=\mathbf{0}_{r}\right\} \subseteq \mathcal{M} \times \mathbb{R}^{d}
$$

For a fixed choice of design parameters $\mathbf{s} \in \mathbb{R}^{d}$ there is a function, called the kinematic constraint mapping (KCM)

$$
F=\hat{F}_{\mathbf{s}}: \mathcal{M} \rightarrow \mathbb{R}^{s}, \quad F\left(g_{1}, \ldots, g_{k}\right)=\hat{F}\left(g_{1}, \ldots, g_{k}, \mathbf{s}\right)
$$

which corresponds to a given manipulator within the family. Consequently, $F^{-1}\left(\mathbf{0}_{r}\right)$ defines a section of D-space, called the configuration space (C-space)

$$
\mathcal{C}:=\left\{\left(g_{1}, \ldots, g_{k}\right) \mid F\left(g_{1}, \ldots, g_{k}\right)=\mathbf{0}_{r}\right\} \subseteq \mathcal{M}
$$

which is associated with the particular manipulator. Note that while we assumed $\mathbf{0}$ to be a regular value of the $\mathrm{D}-\mathrm{KCM} \hat{F}$, it is not necessarily regular for $\hat{F}_{\mathbf{s}}$ for every choice of $\mathbf{s} \in \mathbb{R}^{d}$. The C-space encodes every feasible configuration (reachable point) the manipulator can adopt.

A multi-body system with $t$ joints and $k+1$ links $L_{i}, i=0, \ldots, k$ has $t$ 2-body systems $\left(L_{j-}, L_{j+}\right), j=1, \ldots, t$. These 2-body systems, whose relative displacements are expressed by their joint configuration spaces $\mathcal{C}_{j}$ given in (4.6), together encode all the system's constraints. Assuming one component (e.g. $\left.L_{0}\right)$ is fixed, so $g_{0}=e$, then an alternative definition for the C-space is introduced in terms of displacements of its $t 2$-body systems

$$
\mathcal{C}:=\bigcap_{j=1}^{t} \mathcal{C}_{j} / S E(n)
$$

where $\mathcal{C}_{j}=\left\{\left(g_{0}, \ldots, g_{k}\right) \mid f_{j}\left(g_{j-}, g_{j+}\right)=\right\}, j=1, \ldots, t$ and we factor out free displacement of the base link $L_{0}$. 
It is worth noting here that the construction of $\mathcal{C}$ as the intersection of a collection of 2-body constraints is helpful later in analysing singularities since it ensures a valuable block structure to the Jacobian matrices that determine singularities.

\subsection{Joint variables}

So far, the description of the possible displacements of the links in a multibody system has been expressed in terms of constraints on their free motion in the plane or space. On the other hand, much of the literature on robot kinematics concentrates on the complementary view point of freedom associated with the joints. In (4.10), it was noted that the relative displacements between a pair of links connected by a 1-dof joint can be parametrised by an exponential function associated with an element of the Euclidean Lie algebra or, in other words, as a coset of a 1-parameter subgroup. So, a joint variable describes a relative freedom of a joint, see Figure 4.8a. Of course, the freedom of displacement depends on the type of the joint. An R-joint, for instance, allows a relative rotation about an axis while a $\mathrm{P}$-joint permits a relative translation along an axis between two bodies.

From our point of view, however, we would like to define joint variables explicitly in terms of pose variables, rather than by introducing a new set of independent variables. In the following, we will show how to do this for rotational and translational joints in both planar and spatial cases.

\subsubsection{Planar joint variables}

As pictured in Figure 4.8a, a planar R-joint constrains a pair of components in the plane such that they are only allowed to relatively rotate about an implicit axis perpendicular to this plane. Such a relative rotation can be defined by a variable $\phi$ in terms of the bodies' pose variables of their moving 


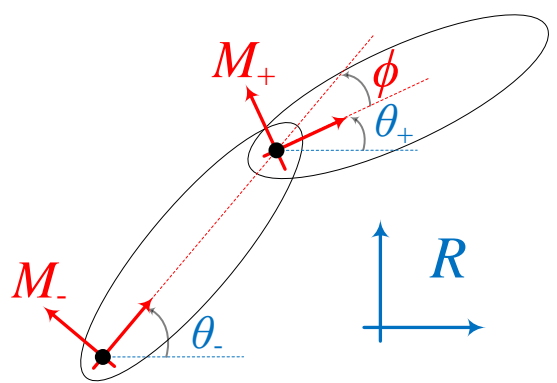

(a) R-joint

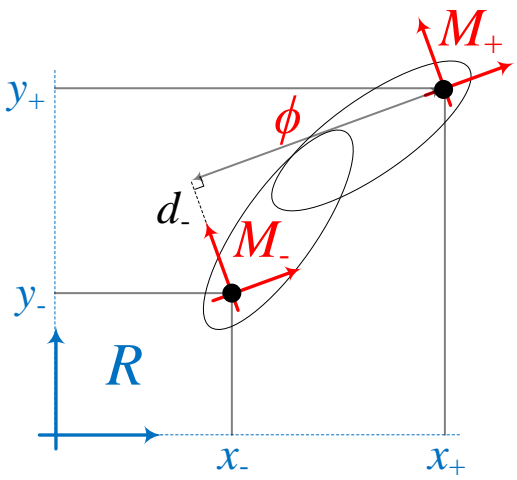

(b) P-joint

Figure 4.8: Planar joint variables

frames $M_{ \pm}$with respect to the reference frame $R$

$$
\phi:=\theta_{+}-\theta_{-}
$$

A planar P-joint, on the other hand, constrains a pair of components in the plane such that they are only allowed to relatively translate along a direction (or line) in the plane, see Figure 4.8b. Thus, a relative translation variable $\phi$ between two adjacent sliding components, whose moving frames are $M_{ \pm}$, is defined by the displacement of the frames' origins along the $\mathrm{P}$ joint with respect to the reference frame $R$. In terms of pose variables, this is

$$
\phi:=\left(\left(x_{+}-x_{-}\right)^{2}+\left(y_{+}-y_{-}\right)^{2}-d_{-}^{2}\right)^{1 / 2}
$$

where $d_{-}$is a design parameter (see Section 4.3.2). This has the disadvantage of not being everywhere differentiable. In practice, it is sufficient and easier to work with $\phi^{2}$. Further, it is clear that we can omit the term involving $d_{-}$ since this simply shifts the origin of the joint variable.

\subsubsection{Spatial joint variables}

A spatial R-joint constrains a pair of components in the 3-dimensional space such that they are only allowed to relatively rotate about an axis with re- 
spect to each other. Suppose the rotating components have moving frames $M_{ \pm}$. Unit quaternions $\mathbf{q}_{ \pm}$may be employed to express the two bodies' orientations with respect to the reference frame $R$ as described in Section 2.4.2. Recall that a rotation by angle $\theta$ about an axis $\mathbf{u}$ is represented by the unit quaternion $\cos \frac{1}{2} \theta+\mathbf{u} \sin \frac{1}{2} \phi$, where $\mathbf{u}$ is a unit 3 -vector. The relative rotation is given by $\phi=\theta_{+}-\theta_{-}$, where $\theta_{ \pm}$are the angles of rotation of $M_{ \pm}$with respect to $R$. This follows because the axes of rotation in the two bodies are constrained to coincide. We may use $\cos \frac{1}{2} \theta$ as the joint variable and so if $\mathbf{q}_{-}=q_{p_{-}^{0}}+q_{p_{-}^{1}} \mathrm{i}+q_{p_{-}^{2}} \mathrm{j}+q_{p_{-}^{3}} \mathrm{k}$ and $\mathbf{q}_{+}=q_{p_{+}^{0}}+q_{p_{+}^{1}} \mathrm{i}+q_{p_{+}^{2}} \mathrm{j}+q_{p_{+}^{3}} \mathrm{k}$ then

$$
\cos \frac{1}{2} \phi=\cos \frac{1}{2}\left(\theta_{+}-\theta_{-}\right)=q_{p_{+}^{0}} q_{p_{-}^{0}}+q_{p_{+}^{1}} q_{p_{-}^{1}}+q_{p_{+}^{2}} q_{p_{-}^{2}}+q_{p_{+}^{3}} q_{p_{-}^{3}}
$$

A spatial P-joint constrains the pose variables of a pair of bodies such that they are only permitted to translate relatively along an axis with respect to each other. As for the planar P-joint, the relevant measure is the relative displacement of the origins of the frames $M_{ \pm}$. In the spatial case, the origins are at $\mathbf{t}_{ \pm}$in the reference frame so that we can use as joint variable

$$
\phi^{2}=\left\|\mathbf{t}_{+}-\mathbf{t}_{-}\right\|_{2}^{2}
$$

Recall from (2.39) that if the displacement of the two bodies relative to $R$ are given by dual quaternions $\breve{\mathbf{q}}_{ \pm}=\mathbf{q}_{p \pm}+\varepsilon \mathbf{q}_{d \pm}$, then $\mathbf{t}_{ \pm}=2 \operatorname{Im}\left(\mathbf{q}_{d \pm} \mathbf{q}_{p \pm}^{*}\right)$ so that $\phi^{2}$ can be written in terms of the pose variables.

\subsection{Actuator space and workspace - input and output maps}

We discussed in Section 4.5 that for a fixed choice of design parameters, a multi-body system has a $\mathrm{KCM} F: \mathcal{M} \rightarrow \mathbb{R}^{s}$, where $\mathcal{M}=S E(n)^{k}$ is the pose space, which defines the $\mathrm{C}$-space $\mathcal{C}=F^{-1}(\mathbf{0})$. As a result, the $\mathrm{C}$-space is 
described in terms of pose variables of all the system's moving components. Pose variables are not distinguished at this stage because we want to model the kinematics of the whole mechanism.

For the given manipulator, there may be several possible choices of actuated joints or inputs. Each actuator may be represented by an associated joint variable. While, mathematically, any joint could be used, practical considerations usually reduce the choices. Similarly, there may be different choices for the end-effector or output. For a typical parallel manipulator, having a platform connected to a base by several serial legs, the typical output is the configuration of the platform.

Let us suppose a choice of actuators is given as a set of $\lambda$ joints; each with just 1-dof. We assume $\lambda \leq \mu$, where $\mu=\operatorname{dim} \mathcal{C}$ is the global mobility given in (4.1), allowing for under-actuation. However, $\lambda>\mu$ would impose constraints on the freedoms of actuated joints. The actuator space (A-space) $\mathcal{A}$, where $\operatorname{dim} \mathcal{A}=\lambda$, is the space parametrised by the actuated joint variables $\phi_{1}, \ldots, \phi_{\lambda}$, also called the inputs, which depend on what quantities actuators have. Although the inputs are distinct from pose variables, they can be expressed in terms of pose variables. In Section 4.6, we discussed how an input can be defined in terms of some relevant pose variables.

For a given choice of end-effector or output link, the workspace (Wspace) $\mathcal{W}$ is parametrised by a subset $u_{1}, \ldots, u_{\nu}$ of pose variables $\hat{\mathbf{u}}:=$ $\left(u_{1}, \ldots, u_{p}, u_{p+1}, \ldots, u_{2 p}, \ldots, u_{k p}\right)$, also called the outputs, which describe poses of the end-effector. Note that $\nu=\operatorname{dim} \mathcal{W} \geq \mu$; we allow inequality since the outputs may not be able to be parametrised by exactly $\mu$ pose variables.

Note that the A-space and W-space can be locally considered as Euclidean spaces, $\mathbb{R}^{\lambda}$ and $\mathbb{R}^{\nu}$, respectively. Associated with these two spaces are functions defined on the manipulator's C-space. The input map $\pi_{a}: \mathcal{C} \rightarrow \mathcal{A}$ is 


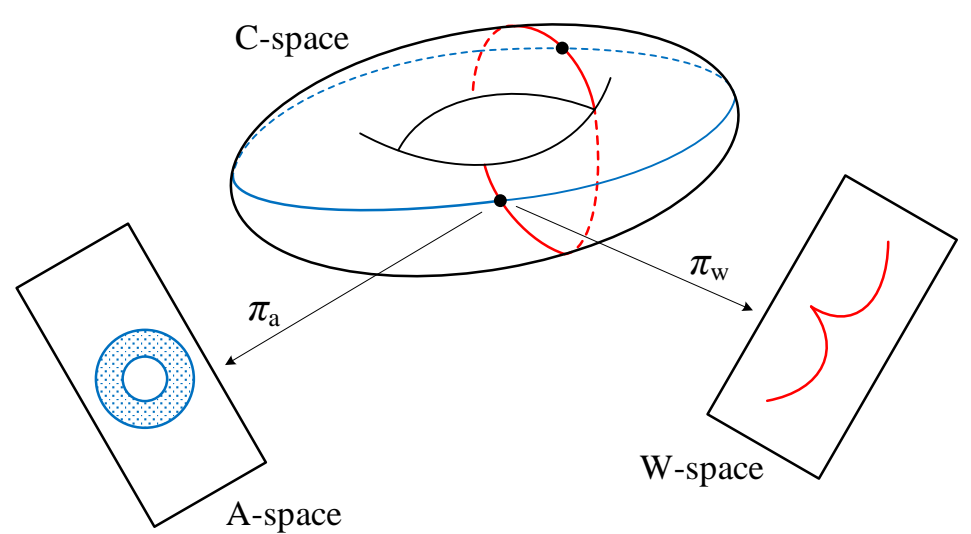

Figure 4.9: Kinematic mappings and the C-space

defined by

$$
\pi_{a}(\hat{\mathbf{u}})=\left(\phi_{1}, \ldots, \phi_{\lambda}\right)
$$

where the joint variables $\phi_{i}, i=1, \ldots, \lambda$ are each functions of the pose variables. The output map $\pi_{w}: \mathcal{C} \rightarrow \mathcal{W}$ is defined by

$$
\pi_{w}(\hat{\mathbf{u}})=\left(u_{1}, \ldots, u_{\nu}\right)
$$

which is the local projection of $\mathcal{C}$ onto the space of pose variables for the end-effector-in general, $\mathcal{W} \subset \mathcal{C}$. Note that $\pi_{a}, \pi_{w}$ are instantaneous and local because they vary from point to point. The overall model is illustrated in Figure 4.9 (see [53]).

In the case of a serial manipulator with 1-dof joints $J_{i}, i=1, \ldots, k$ connecting $L_{i-1}, L_{i}, i=1, \ldots, k$ and with $L_{0}$ the fixed base, the $\mathrm{KCM}$ is a mapping $F: S E(n)^{k} \rightarrow \mathbb{R}^{(p-1) k}, p=\operatorname{dim} S E(n)$. Each of the $k$ sets of $p-1$ components of $F$, say $F_{1}, \ldots, F_{k}$ involves only the pose variables for $L_{i-1}$ and $L_{i}$. Therefore, the C-space $F^{-1}(\mathbf{0})$ is the product of the 1parameter subgroups (or their cosets) defined by the successive joints. Since each of these is parametrised by the corresponding joint variable, the map $\pi_{a}: \mathcal{C} \rightarrow \mathcal{A}$ is a diffeomorphism - a smooth, invertible function, with smooth inverse. 
A significant feature of this kinematics model is that both inputs and outputs are treated as functionally dependent on the C-space and pose variables rather than as independent of each other. In the next Chapter, we show how the input and output mappings combine to address the forward and inverse kinematic problems for manipulators. 


\section{Chapter 5}

\section{Singularities}

In the previous Chapter, a framework for kinematics models of manipulators was established. This involved defining a Kinematic constraint map (KCM) on the space of pose variables, $S E(n)^{k}$, for the moving links. This determines the configuration space for the manipulator. Then input and output maps were introduced.

The goal of this Chapter is to understand the role that the singularities of these maps has on kinematics of individual manipulators and on families of manipulators, with design parameters.

\subsection{Singularities of mappings}

The appropriate mathematical language for singularities is that of differential topology (see, for example, [31]). The objects are (differentiable) manifoldsspaces that are everywhere locally parametrised by a fixed number of parameters, the dimension of the manifold - and differentiable functions or mappings between them. This encompasses the familiar spaces in kinematics such as Lie groups (the Euclidean group), constraints, actuator space, workspace, and families of design parameters.

We start with the definitions of regularity and singularity of a given func- 
tion at a point of its domain:

Definition 5.1. Given a differentiable function $f: M \rightarrow N$ between manifolds and a point $\mathbf{y} \in N$. If for every $\mathbf{x} \in M$ for which $f(\mathbf{x})=\mathbf{y}$ the derivative $d f_{\mathbf{x}}: T_{\mathbf{x}} M \rightarrow T_{\mathbf{y}} N$ of $f$ at $\mathbf{x}$ has maximum rank then $\mathbf{y}$ is called a regular value and each such $\mathbf{x}$ is called a regular point. Otherwise, $\mathbf{y}$ is a singular value and if rank $d f_{\mathbf{x}}$ is less than the maximum, then $\mathbf{x}$ is a singular point.

Note that, for computational purposes, the derivative (which is the linear map that approximates $f$ in a neighbourhood of $\mathbf{x}$ ) can be represented by a Jacobian matrix $J_{\mathbf{x}} f$, which can be computed with respect to any choice of local parametrisations for $M$ and $N$ and whose rank is invariant. Hence, the Jacobian is the matrix representation of this linear map with respect to choices of basis for the tangent spaces at $\mathbf{x}$ and $\mathbf{y}$ determined by the parametrisations.

As a specific example, the KCM defined in (4.28) is a differentiable mapping between manifolds

$$
F: \mathcal{M} \rightarrow \mathbb{R}^{s}
$$

where $\mathcal{M}=S E(n)^{k}$ and $n=2,3$ is the ambient dimension of the manipulator. Given a local parametrisation $\hat{\mathbf{u}}:=\left(u_{1}, \ldots, u_{p}, u_{p+1}, \ldots, u_{2 p}, \ldots, u_{k p}\right)$ on a neighbourhood of $\hat{g}:=\left(g_{1}, \ldots, g_{k}\right) \in \mathcal{M}$, we compute first-order partial derivatives of the $s$ components of the KCM $F$ with respect to $u_{i}$, $i=1, \ldots, m$, where $m=k p$, to attain the $(s \times m)$ Jacobian matrix

$$
J F(\hat{\mathbf{u}})=\left[\frac{\partial f_{j}}{\partial u_{i}}\right]_{i=1, \ldots, m}^{j=1, \ldots, s} \in L\left(\mathbb{R}^{m}, \mathbb{R}^{s}\right)
$$

The Jacobian matrix represents the linear mapping between corresponding tangent spaces

$$
d F_{\hat{g}}: T_{\hat{g}} \mathcal{M} \rightarrow T_{F(\hat{g})} \mathbb{R}^{s}
$$


Note that for $n=3$, we may prefer to use dual quaternions as parametrisations for $S E(3)$ in which case, for each link there are two additional constraint equations of the form (2.38a) and (2.38b). In effect, $\mathcal{M}$ is replaced by $\mathbb{D H}^{k}$ and $s$ is the sum of the total joint constraints and $2 k$.

Returning to the general setting $f: M \rightarrow N$, suppose that $\operatorname{dim} M \geq$ $\operatorname{dim} N$. Two theorems of analysis, which we state here, play a fundamental role (see any standard textbook, such as [93] Pages 35 and 41).

Theorem 5.2 (Inverse Function Theorem). Suppose $F: \mathbb{R}^{m} \rightarrow \mathbb{R}^{m}$ is a continuously differentiable function and $\mathbf{x}_{0} \in \mathbb{R}^{m}$. Then, $f$ has a local differentiable inverse on some neighbourhood of $\mathbf{x}_{0}$ if and only if its Jacobian $J f\left(\mathbf{x}_{0}\right)$ is non-singular.

The strong part of this theorem is the "if" part since it asserts an inverse function exists given only information at the point $\mathbf{x}_{0}$. It is an existence theorem and does not provide a formula for $f^{-1}$.

Theorem 5.3 (Implicit Function Theorem). Suppose $F: \mathbb{R}^{m} \rightarrow \mathbb{R}^{n}$ is a continuously differentiable function, $m \geq n, \mathbf{x}_{0} \in \mathbb{R}^{m}, \mathbf{y}_{0}=f\left(\mathbf{x}_{0}\right)$ and $\operatorname{rank} J f\left(\mathbf{x}_{0}\right)=n$. Let $1 \leq i_{1}<i_{2}<\ldots<i_{n} \leq m$ be indices such that the $(n \times n)$ submatrix of $J f\left(\mathbf{x}_{0}\right)$ formed by the corresponding columns is non-singular. Denote by $j_{1}, \ldots, j_{m-n}$ the remaining indices. Then there are neighbourhoods $U$ of $\left(x_{i_{1}}, \ldots, x_{i_{n}}\right) \in \mathbb{R}^{n}$ and $V$ of $\left(x_{j_{1}}, \ldots, x_{j_{m-n}}\right) \in \mathbb{R}^{m-n}$ and a continuously differentiable function $g: U \rightarrow V$, so that for all $\mathbf{x}=$ $\left(x_{1}, \ldots, x_{m}\right) \in f^{-1}\left(\mathbf{y}_{0}\right)$ with $\pi_{1}(\mathbf{x}) \in U$ and $\pi_{2}(\mathbf{x}) \in V$ (where $\pi_{1}, \pi_{2}$ denote projections onto the relevant subspaces)

$$
\left(x_{j_{1}}, \ldots, x_{j_{m-n}}\right)=g\left(x_{i_{1}}, \ldots, x_{i_{n}}\right)
$$

The theorem says that the inverse image $f^{-1}\left(\mathbf{y}_{0}\right)$ is locally the graph of a 


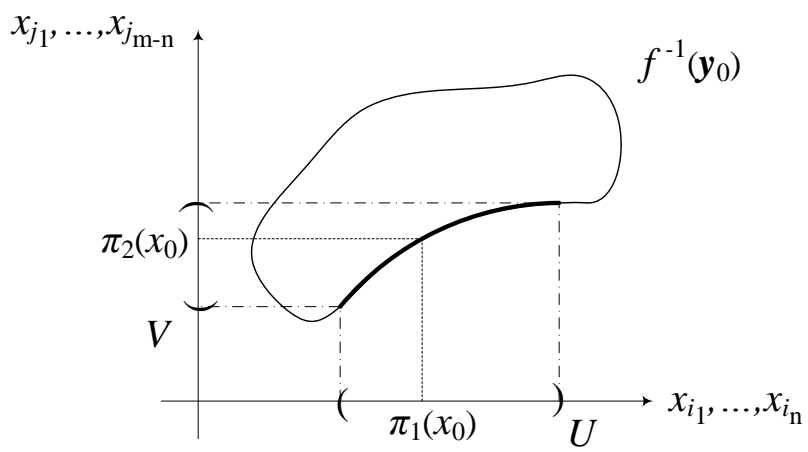

Figure 5.1: An implicit function with associated projections

function in terms of a certain subset of coordinates. There may be several possible choices for $i_{1}, \ldots, i_{n}$ at any given point $\mathbf{x}_{0}$.

Let us state a fundamental theorem of differential topology here (see [31] Page 21)

Theorem 5.4 (Preimage Theorem). If $\mathbf{y}$ is a regular value, so that for any $\mathbf{x} \in P=f^{-1}(\mathbf{y})$ the image of $T_{\mathbf{x}} M$ under the derivative $d f_{\mathbf{x}}$ is the whole of $T_{f(\mathbf{x})} N$, then $P \subset M$ is a submanifold (or possibly empty). Moreover, the codimension of $P$ is the dimension of $N$, or equivalently $\operatorname{dim} P=\operatorname{dim} M-$ $\operatorname{dim} N$.

This follows from Theorem 5.3, by representation $f$ in terms of local parametrisations. The submanifold $P$ can be locally parametrised by a subset of coordinates for $M$. Moreover, it generalises the familiar result of linear algebra concerning the dimension of the solution space of an equation $A \mathbf{x}=\mathbf{b}$, where $A$ has maximum rank. In the case of a $\mathrm{KCM}, F$, if we establish that $\mathbf{0} \in \mathbb{R}^{s}$ is a regular value, then the $\mathrm{C}$-space, $\mathcal{C}=F^{-1}(0)$ will be a manifold of dimension $k p-s$.

Suppose $\mathbf{y} \in N$ is a singular value of $f$. So for at least some point $\mathbf{x} \in M$, with $f(\mathbf{x})=\mathbf{y}, \operatorname{rank} J_{\mathbf{x}} f<\min (\operatorname{dim} M, \operatorname{dim} N)$. Singular values are scarce in the following sense (see [31] Page 39)

Theorem 5.5 (Sard's Theorem). If $f: M \rightarrow N$ is a smooth mapping, then 
the set of singular values, which is a subset of $N$, has measure zero.

Here, a subset of a manifold has measure zero if it can be covered via parametrisations by at most a countably infinite union of hypercubes in $\mathbb{R}^{n}$ whose total volume can be made arbitrarily small. The idea of the proof is that in the neighbourhood of any singular point, $f$ reduces volumes by at least an order of magnitude. Think of the image of an interval $[-r, r]$ under the function $f(x)=x^{2}$. This is $\left[0, r^{2}\right]$ with length $r^{2}$. A property of points in a set that is true except on a set of measure zero is said to hold almost everywhere. So the theorem states that for almost all $\mathbf{y} \in N, \mathbf{y}$ is a regular value. This may lead us to expect that in the "most" cases, the C-space for a manipulator should be a manifold.

\section{$5.2 \quad$ C-space singularities}

\subsubsection{Design parameters}

For a given manipulator architecture, actual models are determined by fixing its design parameters. For serial manipulators, the Denavit-Hartenberg (DH) parameters [89] are widely used to represent the design parameters. The design parameters defined in Chapter 4 are mainly of this type. Another approach [94] uses invariants defined in terms of screw coordinates for successive joints. Among parallel manipulators, the simplest examples are planar 4-R mechanisms for which the design parameters are the link lengths which are DH parameters. However for more complex examples, such as a planar 3-RRR mechanism, in which the platform is connected to multiple links, additional design parametrs must be included (see Section 7.1).

It is reasonable to ask then how the design parameters affect the kinematics of the manipulator. This may involve determining whether certain properties are generic, which is to ask whether the property holds for almost all choices of design parameters. At a more refined level, one may look for $b i$ - 
furcation conditions. These correspond to non-generic choices of parameters across which there may be a significant change of behaviour.

A classical case is the condition of Grashof [4] which distinguishes between planar 4-R mechanisms in terms of rotatability of links and, implicitly between those whose C-space has one or two connected components [47, 95]. It is worth noting, however, that the requirement of symmetry or generation of special motion may be precisely what renders a choice of design parameters non-generic so that, from the engineering point of view, genericity may not be a desirable characteristic.

An important observation is that the separating or bifurcation cases are precisely those for which the C-space has singular points - typically double points where two branches of configurations intersect [47]. A goal of our approach is to generalise this result to other, more complex, families of manipulators.

\subsubsection{Transversality}

Transversality is a concept generalising that of regularity, by which we can seek to answer genericity questions in general. In particular, establishing transversality for functions with respect to certain submanifolds will enable us to deduce not only genericity results, but also information about the dimensions of a hierarchy of singularity sets. For example, if transversality could be verified for a kinematic mapping with respect to submanifolds that encode the rank (or its complement, the corank) of derivatives - in effect, Jacobian matrices of partial derivatives - then the set of configurations at which there is a singularity of a given corank will be a submanifold of a specified dimension. Although not used extensively, these ideas have appeared already in the kinematics literature in the work of Pai [96], Tchon [97] and others. More recently, applications of transversality to the analysis of singularities of mechanisms and related notions of genericity have been explored [98, 99]. 
In this section, we will first review transversality and some of the important theorems it gives rise to. More general consideration of Grashof-type conditions, based on transversality considerations is then illustrated in both negative and positive senses.

Here, a key motivation for transversality is to generalise the submanifold criterion in Section 5.1 to $f^{-1}(Q)$, where $Q \subset N$ is a submanifold. The idea is that for regularity, the image of the derivative $d f_{\mathbf{x}}$ must be the whole of the tangent space, $T_{\mathbf{y}} N$, at the image $f(\mathbf{x})=\mathbf{y}$. Since $T_{\mathbf{y}} Q$ already fills up a subspace of $T_{\mathbf{y}} N$, we now only require the image of $d f_{\mathbf{x}}$ where $f(\mathbf{x}) \in Q$, to span a complement to $T_{\mathbf{y}} Q$.

Definition 5.6. Let $f: M \rightarrow N$ be a differentiable map between (differentiable) manifolds and $Q \subset N$ a submanifold. Define $f$ to be transverse to $Q$ (written $f \pitchfork Q$ ) if for all $\mathbf{x} \in M$ such that $f(\mathbf{x}) \in Q$,

$$
d f_{\mathbf{x}}\left(T_{\mathbf{x}} M\right)+T_{f(\mathbf{x})} Q=T_{f(\mathbf{x})} N
$$

In this case, an extension of Sard's Theorem states that $P=f^{-1}(Q)$ is a submanifold of $M$ and $\operatorname{codim} P=\operatorname{codim} Q$ (see, for example, [31]). A corollary of that theorem is the following [31]

Theorem 5.7 (Parametric Transversality Theorem). Given a family of functions $F: M \times S \rightarrow N$, where $S$ is a manifold of parameters, then for each fixed $\mathbf{s} \in S$ there is a function $F_{\mathbf{s}}: M \rightarrow N$ where, for $\mathbf{x} \in M, F_{\mathbf{s}}(\mathbf{x})=F(\mathbf{x}, \mathbf{s})$. If $Q \subset N$ is a submanifold and $F$ $Q$ then for almost all $\mathbf{s} \in S, F_{\mathbf{s}} \pitchfork Q$.

This has direct interpretations in kinematics. We could take $M$ as the Cspace for a family of mechanisms, $S$ as the space of design parameters and $N$ the workspace, for example the Euclidean group. In this case, $Q$ may be a submanifold that characterises some desired geometry for the end-effector or platform such as that it has a fixed orientation. 
Alternatively, suppose the D-KCM $\hat{F}: \mathcal{M} \times \mathbb{R}^{d} \rightarrow \mathbb{R}^{s}$ explained in Section 4.5 where $M=\mathcal{M}$ is the pose space given in (2.46) for a family of manipulators, $S=\mathbb{R}^{d}$ the design space and $N=\mathbb{R}^{s}$ the constraint space. Here, the role of $Q$ is more limited, as there is typically only one relevant choice of values (usually zero) for each equation, so that $Q=\{\mathbf{0}\}$. This corresponds to a special case of Theorem 5.7, when $Q=\left\{\mathbf{y}_{0}\right\} \subset \mathbb{R}^{s}$, that is a single point or 0 -dimensional submanifold. In this case, $\hat{F} \pitchfork Q$ if and only if $\mathbf{y}_{0}$ is a regular value of $\hat{F}$, since the second term in the defining condition (5.4) reduces to $\{\mathbf{0}\}$. That is, the Jacobian has maximum rank for all $(\hat{\mathbf{u}}, \mathbf{s}) \in \mathcal{M} \times \mathbb{R}^{d}$ for which $\hat{F}(\hat{\mathbf{u}}, \mathbf{s})=\mathbf{y}_{0}$. In this case, we can conclude that for almost all $\mathbf{s} \in \mathbb{R}^{d}$

$$
\mathcal{C}_{\mathbf{s}}:=\hat{F}_{\mathrm{s}}^{-1}\left(\mathbf{y}_{0}\right) \subset \mathcal{M}
$$

is a $C^{\infty}$ submanifold with $\operatorname{dim} \mathcal{C}_{\mathbf{s}}=m-s$ (or else an empty set). In the context of the KCM, note that $\hat{F}_{\mathbf{s}}$ is the KCM given in (4.28) thus $\mathcal{C}_{\mathbf{s}}$ is the C-space for the specific choice of design parameters, $\mathbf{s} \in \mathbb{R}^{d}$, as in (4.29).

\subsubsection{Grashof-type conditions}

It is reasonable to pose the question whether we can find Grashof-type conditions on the design space for singularities of the C-space. We can undertake some dimensional analysis to indicate what is likely. The set of points where the corank of the Jacobian matrix of a mapping $f: M \rightarrow N$ between manifolds is positive is called its singular set, denoted as follows

$$
\Sigma F:=\left\{\mathbf{x} \mid \operatorname{rank} J_{\mathbf{x}} f<\min (\operatorname{dim} M, \operatorname{dim} N)\right\}
$$

This may be further refined by considering the sets of matrices of a fixed corank. Set $\operatorname{dim} M=m, \operatorname{dim} N=n$ and let us suppose that $m \geq n$; we are interested (locally) in the map $J f: M \rightarrow L\left(\mathbb{R}^{m}, \mathbb{R}^{n}\right)$ where the codomain is the set of linear functions between $\mathbb{R}^{m}$ and $\mathbb{R}^{n}$, or $(n \times m)$ matrices. It is 
then defined that $\Sigma^{\kappa}=\left\{A \in L\left(\mathbb{R}^{m}, \mathbb{R}^{n}\right) \mid \operatorname{corank} A=\kappa\right\}$. It can be shown (see, for example, [33]) that these sets are submanifolds, satisfying

$$
\operatorname{codim} \Sigma^{\kappa}=\kappa(m-n+\kappa)
$$

In the case that $J f \pitchfork \Sigma^{\kappa}$, we would have $\Sigma^{\kappa} f=J f^{-1}\left(\Sigma^{\kappa}\right)$ a submanifold of $M$ of the same codimension; in the simplest case, $\kappa=1$, this is $m-n+1$.

Let us apply this to the setting of D-KCM $\hat{F}: \mathcal{M} \times \mathbb{R}^{d} \rightarrow \mathbb{R}^{s}$. Under the assumption of transversality on $\hat{F}$, the D-space $\mathcal{D}=\hat{F}^{-1}(\mathbf{0}) \subset \mathcal{M} \times \mathbb{R}^{d}$ is a submanifold of codimension $s$. For each $\mathbf{s} \in \mathbb{R}^{d}$ we have a C-space $\mathcal{C}=\mathcal{D} \cap(\mathcal{M} \times\{\mathbf{s}\}) \subset \mathcal{M} \times\{\mathbf{s}\}$, with the same codimension (provided it is a submanifold). Each $\mathcal{C}$ may have singularities but the simplest singularity set $\Sigma^{1} F$, where $F=\hat{F}_{\mathrm{s}}$ has codimension $m-s+1$. Since the sum of the codimensions of $\mathcal{C}$ and $\Sigma^{1} F$ in $\mathcal{M}$ is $(m-s+1)+s>m$ we would not typically expect a given $\mathcal{C}$ to have any singularities.

On the other hand, in the D-space, the singularity set of interest is $\Sigma_{1}^{1} \hat{F}=J_{1} \hat{F}^{-1}\left(\Sigma^{1}\right)$, where $J_{1} \hat{F}: \mathcal{M} \times \mathbb{R}^{d} \rightarrow L\left(\mathbb{R}^{m}, \mathbb{R}^{s}\right)$ is the Jacobian map, which treats $\mathbb{R}^{d}$ as a parameter space rather than as variables to be differentiated. Transversality then gives codim $\Sigma_{1}^{1} \hat{F}=m-s+1$. Under the additional assumption that $\mathcal{D}$ and $\Sigma_{1}^{1} \hat{F}$ meet transversely, their intersection is a submanifold satisfying

$$
\operatorname{codim}\left(\mathcal{D} \cap \Sigma_{1}^{1} \hat{F}\right)=\operatorname{codim} \mathcal{D}+\operatorname{codim} \Sigma_{1}^{1} \hat{F}=m+1
$$

In other words, in $\mathcal{M} \times \mathbb{R}^{d}$, the singularity set is defined by $m+1$ simultaneous (non-linear) equations. If it is possible to eliminate the $m$ pose variables we should arrive at just one equation in the $d$ design parameters. If such a condition can be found, we call it the Grashof-type condition for the family of manipulators. Note that there should be a single condition, independent of the number of links, joints or design parameters. We give 
examples in Chapters 6-9.

\subsection{Input and output singularities}

While the KCM and associated C-space is a fundamental problem in kinematics, there are two central practical problems in this field, namely the inverse kinematic problem (IKP) and forward kinematic problem (FKP). Solving these, or showing they cannot be solved, is closely related to singularity analysis. Most of the other approaches to kinematic singularities, like those of Gosselin and Angeles [1] and Zlatanov et al. [2], address these two problems.

In simple terms, suppose a manipulator has a chosen set of actuator joints and a given end-effector or output. The FKP requires to determine the endeffector pose given the joint inputs. Conversely, the IKP asks how to choose the joint inputs in order to achieve a given output (end-effector pose).

In some cases, one or other of these may be relatively straightforward. For example, in a serial manipulator the FKP can be uniquely solved-the endeffector pose may be computed as a product of exponentials in terms of the joint variables. The Gough-Stewart (GS) platform (see, for example, [100, 101, 102]) is a well-known type of parallel manipulator in which a platform is supported by 6-RPU legs with P-joints actuated, and the axes of the Pjoints pass through the centres of the R- and U-joints in each leg. For a given pose of the platform, the leg lengths - effectively the joint variables for the P-joints - are able to be uniquely found so the IKP is solved. On the other hand, the IKP for serial manipulators with six degrees of freedom only has closed-form solution for special (wrist-separated) architectures [70], while the FKP for the GS platform may have many solutions [103, 104].

There are also instantaneous versions of these problems. Let us suppose a pose of the end-effector is given, then the instantaneous IKP tells us what joint velocities are required to generate a given end-effector (generalised) velocity. On the other hand, let us assume the velocities of actuators are 


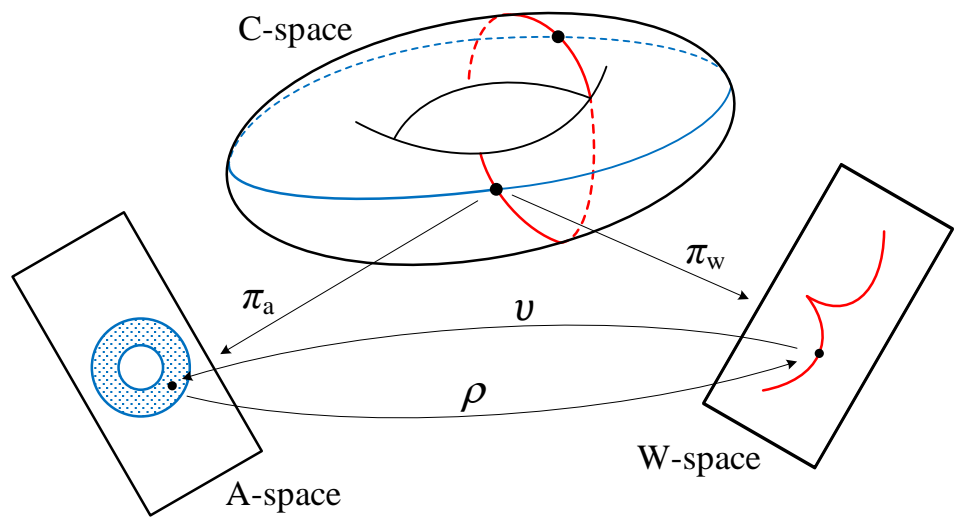

Figure 5.2: Forward and inverse kinematic mappings

given. Then, the instantaneous FKP indicates what the end-effector velocity is.

In this section, we are going to develop the theory to incorporate inputs and outputs, based on choice of actuated joints (inputs) and on the manipulator's workspace or end-effector (outputs).

In the language of C-space, input and output maps, the relationships in each direction between the inputs and the outputs represent the forward kinematic problems $(\mathrm{FKP}) \rho: \mathcal{A} \rightarrow \mathcal{W}$ and inverse kinematic problems (IKP) $v: \mathcal{W} \rightarrow \mathcal{A}$. The $\rho, v$ are relations between $\mathcal{A}, \mathcal{W}$, but not necessarily welldefined functions. These are illustrated in Figure 5.2.

As noted previously, in much of the manipulator singularity literature the kinematic models have been encoded by an implicit function

$$
F(\boldsymbol{\theta}, \mathbf{x})=\mathbf{0}
$$

involving both input and output variables. The ability to express inputs $\boldsymbol{\theta}$ or outputs $\mathbf{x}$ explicitly in terms of the other can generally only be solved locally and requires the conditions for the Implicit Function Theorem 5.3, which we stated in Section 5.1, to be met. However, it is known that analysing this implicit function alone does not provide full information about manipulator singularities in the parallel case, because equation (5.9) does not capture 
information about non-actuated joints.

In our model, the $\mathrm{C}$-space is known via the $\mathrm{KCM}$, then the $\mathrm{A}$-space and W-space can be related together by means of the input and output maps. As is clear from Figure 5.2, the forward and inverse relations can be determined in terms of the input and output maps, provided these have well defined (local) inverses

$$
\rho=\pi_{a}^{-1} \circ \pi_{w}, \quad v=\pi_{w}^{-1} \circ \pi_{a}
$$

The existence of the inverses is a local question. Assume, for the moment, that $\operatorname{dim} \mathcal{A}=\operatorname{dim} \mathcal{C}=\operatorname{dim} \mathcal{W}^{1}$. Then, by the Inverse Function Theorem 5.2, $\pi_{a}$ or $\pi_{w}$ has a local inverse at some $\hat{\mathbf{u}} \in \mathcal{C}$ if and only if its Jacobian at that point is non-singular. Therefore, the input and output singularities are critical to the solutions of the FKP and IKP. We observed in Section 4.7 that for a serial manipulator, the C-space is locally isomorphic to the actuator space so that $\pi_{a}$ is always invertible and the FKP is well-posed.

In order to detect the input and the output singularities, we apply an extension of the method of constrained optimisation which we state here in terms of mappings between Euclidean spaces, but which generalises to smooth manifolds.

Theorem 5.8. Let $F: \mathbb{R}^{m} \rightarrow \mathbb{R}^{s}(m>s)$ and suppose that $\mathbf{0} \in \mathbb{R}^{s}$ is a regular value so that $\mathcal{C}:=F^{-1}(\mathbf{0})$ is a smooth manifold of dimension $\mu=$ $m-s$. Given a differentiable function $g: \mathbb{R}^{m} \rightarrow \mathbb{R}^{p}(p \geq \mu)$ then $\hat{\mathbf{u}} \in \mathcal{C}$ is a singular point of $\left.g\right|_{\mathcal{C}}: \mathcal{C} \rightarrow \mathbb{R}^{p}$ if and only if rank $J G(\hat{\mathbf{u}})<m$, where $G:=(g, F)$.

The theorem follows from the fact that if $\hat{\mathbf{u}} \in \mathcal{C}$ then $T_{\hat{\mathbf{u}}} \mathcal{C}=\operatorname{ker} J F(\hat{\mathbf{u}})$, so the rank of $J G(\hat{\mathbf{u}})$ falls if and only if the nullity of the derivative of $\left.g\right|_{\mathcal{C}}$ at $\hat{\mathbf{u}}$ increases. This seems to be a standard result but a reference has not been identified.

\footnotetext{
${ }^{1}$ This corresponds to the regular type of manipulators. Of course, there are other types to be considered such as redundant manipulators where $\operatorname{dim} \mathcal{A}>\operatorname{dim} \mathcal{C}$. This can be subject for future work.
} 
In the case $p=\mu$, the $J G(\hat{\mathbf{u}})$ is an $(m \times m)$ square matrix whose first $\mu$ rows are associated to first-order partial derivatives of $g$ whereas the last $m-\mu$ rows are associated to $F$ given in (5.2). In this case, rank deficiency requires simply

$$
\operatorname{det} J G(\hat{\mathbf{u}})=0
$$

We can apply Theorem 5.8 when $F$ is a KCM and $g$ is the input map $\pi_{a}$ given in (4.35). Then, equation (5.11) will correspond to a configuration where some actuator joint velocity cannot be realised. Therefore, the FKP cannot be solved instantaneously or locally. That is, the inputs are instantaneously fixed, but the output will not vanish and thus the end-effector will admit instantaneous displacement. On the other hand, if the output map $\pi_{w}$ given in (4.36) is involved, equation (5.11) will correspond to some instantaneous input (joint velocity) which does not produce an instantaneous displacement of the end-effector.

\subsubsection{Ceva's theorem}

In Chapter 7, examples show that input and output singularities occur if some particular geometrical conditions are instantaneously satisfied within the whole mechanism. One important example that occurs for planar manipulators is contained in the following theorem.

Ceva's theorem can be found in many texts on Euclidean geometry, for instance [105]. This theorem is essential to our discussion on the input and the output singularities because it reveals a geometrical condition which will facilitate detecting a singular configuration within a mechanism. We state the theorem here in terms of angle division, rather than the more common side division.

Let us consider a triangle $A B C$ in $2 \mathrm{D}$ with interior angles $\alpha, \beta, \gamma$ as illustrated in Figure 5.3. Three lines $\ell_{a}, \ell_{b}, \ell_{c}$ pass through the vertices $A, B, C$ 


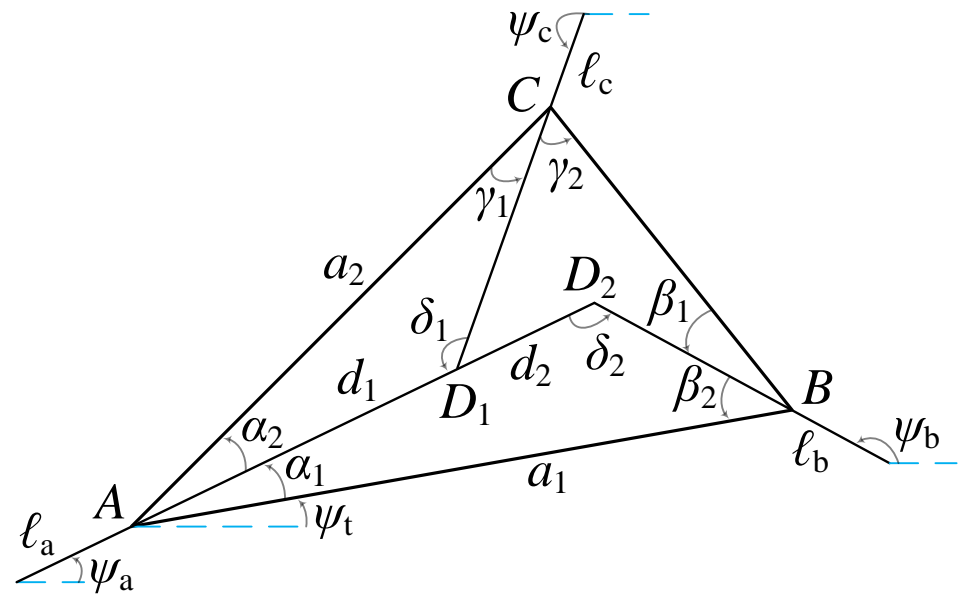

Figure 5.3: A triangle considered for Ceva's theorem

dividing each internal angle into two subangles

$$
\alpha=\alpha_{1}+\alpha_{2}, \quad \beta=\beta_{1}+\beta_{2}, \quad \gamma=\gamma_{1}+\gamma_{2}
$$

Note that the angles should be measured with a consistent orientation. The first subangle may be chosen between 0 and $\pi$. If the line is wholly exterior to the triangle then the second subangle will be negative.

One classical form of Ceva's Theorem states that $\ell_{a}, \ell_{b}, \ell_{c}$ are concurrent if and only if

$$
\sin \alpha_{1} \sin \beta_{1} \sin \gamma_{1}=\sin \alpha_{2} \sin \beta_{2} \sin \gamma_{2}
$$

It will be more helpful for us to present this condition in a terms of design parameters and external angles of the triangle, which will correspond to the platform of a planar parallel mechanism.

Theorem 5.9 (Ceva's Theorem). With the notation above, suppose that $\ell_{a}, \ell_{b}, \ell_{c}$ make angles $\psi_{a}, \psi_{b}, \psi_{c}$, while the base of triangle $A B C$ makes an angle $\psi_{t}$, to the horizontal in a fixed reference frame. Let $\ell_{a}, \ell_{b}$ meet at $D_{2}$, a distance $d_{2}$ from $A$, and $\ell_{a}, \ell_{c}$ meet at $D_{1}$, a distance $d_{1}$ from $A$. Then, 
$D_{1}=D_{2}$ (so the three lines are concurrent) if and only if

$$
a_{1} \sin \left(\psi_{a}-\psi_{c}\right) \sin \left(\psi_{b}-\psi_{t}\right)=a_{2} \sin \left(\psi_{a}-\psi_{b}\right) \sin \left(\psi_{c}-\alpha-\psi_{t}\right)
$$

Proof. By looking at Figure 5.3, we can obtain the following relations between the angles

$$
\begin{array}{r}
\delta_{1}-\psi_{a}+\psi_{c}=2 \pi, \\
\beta_{2}-\psi_{t}+\psi_{b}=\pi \Rightarrow \psi_{b}-\psi_{t}=\pi-\beta_{2}, \\
\psi_{a}+\delta_{2}=\psi_{b}, \\
\psi_{t}+\alpha+\gamma_{1}=\psi_{c}-\pi \Rightarrow \pi+\gamma_{1}=\psi_{c}-\alpha-\psi_{t}
\end{array}
$$

These hold independent of whether the lines are concurrent. Taking sines from both sides of equations (5.14a-5.14d) gives

$$
\begin{array}{r}
\sin \delta_{1}=\sin \left(\psi_{a}-\psi_{c}\right), \\
\sin \beta_{2}=\sin \left(\psi_{b}-\psi_{t}\right), \\
\sin \delta_{2}=-\sin \left(\psi_{a}-\psi_{b}\right), \\
\sin \gamma_{1}=-\sin \left(\psi_{c}-\alpha-\psi_{t}\right)
\end{array}
$$

Therefore, equation (5.13) can be rewritten

$$
a_{1} \sin \delta_{1} \sin \beta_{2}=a_{2} \sin \delta_{2} \sin \gamma_{1}
$$

Now, the law of sines gives

$$
\begin{aligned}
\frac{\sin \delta_{1}}{a_{2}} & =\frac{\sin \gamma_{1}}{d_{1}} \\
\frac{\sin \delta_{2}}{a_{1}} & =\frac{\sin \beta_{2}}{d_{2}}
\end{aligned}
$$


Hence, equation (5.13) is equivalent to

$$
d_{2} \sin \delta_{1} \sin \delta_{2}=d_{1} \sin \delta_{1} \sin \delta_{2}
$$

or $d_{1}=d_{2}$, as required.

Equation (5.13) will be employed in Sections 7.1 and 7.2 as a tool to explain the input and the output singularities. 


\section{Chapter 6}

\section{Classical planar mechanisms}

In Chapters 2-5, a comprehensive model for manipulator kinematics has been set up. This is based on the observation that the motion of all the links in a manipulator should be included, independent of the choice of inputs and outputs. The constraints on motion are completely modelled by the joints connecting pairs of linkages. From these constraints, equations can be written down defining the full configuration space (C-space) of the manipulator. Moreover, these equations depend on design parameters for the manipulator architecture. In addition, for any choice of inputs (actuated joints) and outputs (end-effector), input and output mappings can be defined on the C-space.

In the following Chapters, we introduce a range of examples to illustrate how the model can be used to identify singularities of the C-space and the input/output maps. These range from classical planar linkages such as the planar 4-bar to spatial platforms, and also include an analysis of singularities for a planar geared mechanism. 


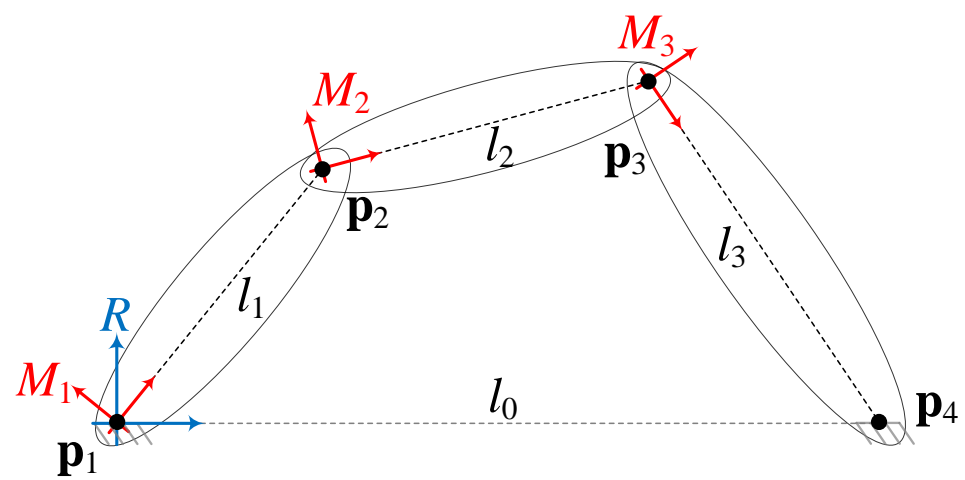

Figure 6.1: 4-R linkage with associated frames and design parameters

\subsection{Planar 4-R linkage}

As illustrated in Figure 6.1, this linkage is made up of four rigid links $(k=4)$ and has four design parameters $l_{i}, i=0,1,2,3$ which are the lengths of its links. Note that $l_{0}$ is the base which we will regard as fixed. The linkage is fundamental in the early history of mechanisms and has been the subject of extensive analysis [106, 4, 107, 47, 59]. This example provides a test case for the model since the relevant singularities are already available in the literature (for example see [47, 1, 2, 54]).

Displacement of each moving component belongs to $S E(2)$ and is represented by three pose variables $\left(x_{i}, y_{i}, \theta_{i}\right)^{T}, i=1,2,3$. From Section 4.3.1, the geometric representation of a planar R-joint is given by coincidence of a point in each connected link. With a choice of moving frames $M_{i}, i=1,2,3$ and reference frame $R$, the body coordinates of each joint $\mathbf{p}_{i} \in \mathbb{R}^{2}, i=1,2,3,4$ with respect to the frames ${ }^{1}$ of links it connects are

$$
\begin{aligned}
& \mathbf{p}_{1}^{R}=(0,0)^{T}, \quad \mathbf{p}_{2}^{1}=\left(l_{1}, 0\right)^{T}, \quad \mathbf{p}_{3}^{2}=\left(l_{2}, 0\right)^{T}, \quad \mathbf{p}_{4}^{3}=\left(l_{3}, 0\right)^{T}, \\
& \mathbf{p}_{1}^{1}=(0,0)^{T}, \quad \mathbf{p}_{2}^{2}=(0,0)^{T}, \quad \mathbf{p}_{3}^{3}=(0,0)^{T}, \quad \mathbf{p}_{4}^{R}=\left(l_{0}, 0\right)^{T}
\end{aligned}
$$

We now obtain the reference-frame coordinates of the joints in both con-

\footnotetext{
${ }^{1}$ The superscript identifies the frame.
} 
nected links, by using (2.6)

$$
\begin{array}{llll}
\mathbf{P}_{1}^{R}=A_{R} \mathbf{p}_{1}^{R}+\mathbf{a}_{R}, & \mathbf{P}_{2}^{1}=A_{1} \mathbf{p}_{2}^{1}+\mathbf{a}_{1}, & \mathbf{P}_{3}^{2}=A_{2} \mathbf{p}_{3}^{2}+\mathbf{a}_{2}, & \mathbf{P}_{4}^{3}=A_{3} \mathbf{p}_{4}^{3}+\mathbf{a}_{3}, \\
\mathbf{P}_{1}^{1}=A_{1} \mathbf{p}_{1}^{1}+\mathbf{a}_{1}, & \mathbf{P}_{2}^{2}=A_{2} \mathbf{p}_{2}^{2}+\mathbf{a}_{2}, & \mathbf{P}_{3}^{3}=A_{3} \mathbf{p}_{3}^{3}+\mathbf{a}_{3}, & \mathbf{P}_{4}^{R}=A_{R} \mathbf{p}_{4}^{R}+\mathbf{a}_{R}
\end{array}
$$

Equation (4.12) can now be applied to each joint in turn

- joint 1 connecting $L_{1}$ to $L_{\mathbf{0}}: \mathbf{P}_{1}^{R}-\mathbf{P}_{1}^{1}=\mathbf{0} \Rightarrow \mathbf{a}_{1}-\mathbf{p}_{1}^{R}=\mathbf{0}$

- joint 2 connecting $L_{2}$ to $L_{1}: \mathbf{P}_{2}^{1}-\mathbf{P}_{2}^{2}=\mathbf{0} \Rightarrow A_{1} \mathbf{p}_{2}^{1}+\mathbf{a}_{1}-\mathbf{a}_{2}=\mathbf{0}$

- joint 3 connecting $L_{3}$ to $L_{2}: \mathbf{P}_{3}^{2}-\mathbf{P}_{3}^{3}=\mathbf{0} \Rightarrow A_{2} \mathbf{p}_{3}^{2}+\mathbf{a}_{2}-\mathbf{a}_{3}=\mathbf{0}$

- joint 4 connecting $L_{\mathbf{0}}$ to $L_{3}: \mathbf{P}_{4}^{3}-\mathbf{P}_{4}^{R}=\mathbf{0} \Rightarrow A_{3} \mathbf{p}_{4}^{3}+\mathbf{a}_{3}-\mathbf{p}_{4}^{R}=\mathbf{0}$

Altogether, these give eight scalar joint constraint equations, so the resulting $\mathrm{KCM} F: S E(2)^{3} \rightarrow \mathbb{R}^{8}$ can be written in terms of the parametrisation in $(2.28)$

$$
\begin{gathered}
\left(x_{i}, y_{i}, \theta_{i}\right)_{i=1,2,3} \mapsto\left[-x_{1},-y_{1}, x_{1}-x_{2}+l_{1} c_{1}, y_{1}-y_{2}+l_{1} s_{1}, x_{2}-x_{3}+l_{2} c_{2}\right. \\
\left.y_{2}-y_{3}+l_{2} s_{2}, x_{3}-l_{0}+l_{3} c_{3}, y_{3}+l_{3} s_{3}\right]^{T}
\end{gathered}
$$

where $c_{i}=\cos \theta_{i}$ and $s_{i}=\sin \theta_{i}, i=1,2,3$. The null-set $F^{-1}(\mathbf{0})$ defines the $\mathrm{C}$-space for the 4-R linkage. 


\subsubsection{C-space singularities and Grashof-type conditions}

Taking partial derivatives of (6.3) with respect to the pose variables yields an $(8 \times 9)$ Jacobian matrix

$$
J_{1} F=\left[\begin{array}{ccccccccc}
x_{1} & y_{1} & x_{2} & y_{2} & x_{3} & y_{3} & \theta_{1} & \theta_{2} & \theta_{3} \\
-1 & 0 & 0 & 0 & 0 & 0 & 0 & 0 & 0 \\
0 & -1 & 0 & 0 & 0 & 0 & 0 & 0 & 0 \\
1 & 0 & -1 & 0 & 0 & 0 & -l_{1} s_{1} & 0 & 0 \\
0 & 1 & 0 & -1 & 0 & 0 & l_{1} c_{1} & 0 & 0 \\
0 & 0 & 1 & 0 & -1 & 0 & 0 & -l_{2} s_{2} & 0 \\
0 & 0 & 0 & 1 & 0 & -1 & 0 & l_{2} c_{2} & 0 \\
0 & 0 & 0 & 0 & 1 & 0 & 0 & 0 & -l_{3} s_{3} \\
0 & 0 & 0 & 0 & 0 & 1 & 0 & 0 & l_{3} c_{3}
\end{array}\right]
$$

which, following row operations and deletions of rows and columns with leading 1 s, can be reduced to a $(2 \times 3)$ matrix that is sufficient to determine rank deficiency

$$
J_{1} F^{\mathrm{red}}=\left[\begin{array}{ccc}
-l_{1} s_{1} & -l_{2} s_{2} & -l_{3} s_{3} \\
l_{1} c_{1} & l_{2} c_{2} & l_{3} c_{3}
\end{array}\right]
$$

In this case, the translational pose variables have been eliminated so that the singularity condition depends only on the "free" rotational parameter for each joint and the design parameters. The singular set $\Sigma_{1}^{1} F$ (defined in Section 5.2 .3$)$ is determined by all maximal $(2 \times 2)$ non-zero 1 -minors of $(6.4)$ vanishing simultaneously

$$
-l_{1} l_{2} s_{1,2}=-l_{1} l_{3} s_{1,3}=-l_{2} l_{3} s_{2,3}=0
$$

where $s_{i, j}=\sin \left(\theta_{i}-\theta_{j}\right)$. These equations hold if

$$
\theta_{i}=\theta_{j}+\eta_{i j} \pi, i, j=1,2,3 \quad \eta_{i j} \in \mathbb{Z}
$$




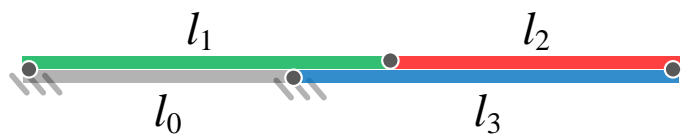

Figure 6.2: A C-space singularity for 4-R linkage

Geometrically, the three mobile links must be parallel or antiparallel. It follows that $c_{j}= \pm c_{i}$ and $s_{j}= \pm s_{i}$ for each pair $i, j$. We are only interested in points in the C-space. Substituting equation (6.5) in (6.3) and setting $F=\mathbf{0}$ results in

$$
\begin{aligned}
& \left(l_{1} \pm l_{2} \pm l_{3}\right) s_{1}=0 \\
& \left(l_{1} \pm l_{2} \pm l_{3}\right) c_{1}=l_{0}
\end{aligned}
$$

Assuming $l_{0} \neq 0$, we obtain from equation (6.6a) that $\theta_{1}=0$ or $\pi$ and thus from equation $(6.6 \mathrm{~b})$

$$
l_{0} \pm l_{1} \pm l_{2} \pm l_{3}=0
$$

which are Grashof-type conditions. The conditions correspond to eight hyperplanes in the design space. These separate the positive orthant into regions characterised by the possible motion of the moving links [107]. Only when one of the cases in equation (6.7) holds is there a C-space singularity and geometrically this corresponds to the 4-R reaching a flat configuration, as illustrated in Figure 6.2. Although equation (6.7) is well known, we include its derivation as a case study to validate the approach.

To illustrate the application of Theorem 5.7 we consider the property "that the C-space for a given family of mechanisms is a manifold" is generic. We will revisit the theorem for a slightly more complicated case in Section 6.3. By adding design parameters $l_{j}, j=1,2,3$ to (6.3) and allowing them to vary, the $\mathrm{D}-\mathrm{KCM}$ associated to the 4-R mechanism can be obtained

$$
\hat{F}\left(\theta_{i}, x_{i}, y_{i}, l_{j}\right)_{i=1, \ldots 3}^{j=0, \ldots 3}: S E(2)^{3} \times \mathbb{R}^{4} \rightarrow \mathbb{R}^{8}
$$


Here, design parameters are allowed to vary. Hence, the extended Jacobian $J \hat{F}$ with respect to both pose variables and design parameters is an $(8 \times 13)$ matrix. By doing row operations, we can obtain the row-reduced echelon form (rref)

$$
J \hat{F}^{\mathrm{rref}}=\left[\begin{array}{ccccccccccccc}
1 & 0 & 0 & 0 & 0 & 0 & 0 & 0 & 0 & 0 & 0 & 0 & 0 \\
0 & 1 & 0 & 0 & 0 & 0 & 0 & 0 & 0 & 0 & 0 & 0 & 0 \\
0 & 0 & 1 & 0 & 0 & 0 & 0 & l_{1} s_{1} & 0 & 0 & -c_{1} & 0 & 0 \\
0 & 0 & 0 & 1 & 0 & 0 & 0 & -l_{1} c_{1} & 0 & 0 & -s_{1} & 0 & 0 \\
0 & 0 & 0 & 0 & 1 & 0 & 0 & l_{1} s_{1} & l_{2} s_{2} & 0 & -c_{1} & -c_{2} & 0 \\
0 & 0 & 0 & 0 & 0 & 1 & 0 & -l_{1} c_{1} & -l_{2} c_{2} & 0 & -s_{1} & -s_{2} & 0 \\
0 & 0 & 0 & 0 & 0 & 0 & 1 & l_{1} s_{1} & l_{2} s_{2} & l_{3} s_{3} & -c_{1} & -c_{2} & -c_{3} \\
0 & 0 & 0 & 0 & 0 & 0 & 0 & -l_{1} c_{1} & -l_{2} c_{2} & -l_{3} c_{3} & -s_{1} & -s_{2} & -s_{3}
\end{array}\right]
$$

This clearly has full rank because the last row cannot be identically zerothere is no solution to the simultaneous equations $\sin \theta=\cos \theta=0$.

We have thus established that $\hat{F} \pitchfork\{\mathbf{0}\}$ and thus for almost all $\left(l_{0}, \ldots, l_{3}\right)^{T}$ $\in \mathbb{R}^{4}$ the $\mathrm{C}$-space has no singularity. Of course, in this case we already know that $\mathcal{C}$ is non-singular unless the Grashof-type condition given in (6.7) holds for the design parameters. This tells us directly that $\mathcal{C}$ is non-singular except on the closed subset of the positive orthant defined by the union of these hyperplanes.

As an aside, we note that Grashof-type conditions can be found by solving the $\mathrm{KCM}, F=\mathbf{0}$, along with the equations that determine positive corank for its Jacobian $J_{1} F^{\text {red }}$ given in (6.4), namely that all the 1-minors vanish simultaneously. Since all the equations are algebraic in $x_{i}, y_{i}, c_{i}, s_{i}, i=1,2,3$ and subject to the additional conditions $c_{i}^{2}+s_{i}^{2}=1$, we may use Gröbner bases (see any standard textbook, such as [108]) to eliminate the pose variables and obtain

$$
l_{0}^{8}-4 l_{0}^{6} l_{1}^{2}+6 l_{0}^{4} l_{1}^{4}-4 l_{0}^{2} l_{1}^{6}-4 l_{0}^{6} l_{2}^{2}+4 l_{0}^{4} l_{1}^{2} l_{2}^{2}+4 l_{0}^{2} l_{1}^{4} l_{2}^{2}+6 l_{0}^{4} l_{2}^{4}+4 l_{0}^{2} l_{1}^{2} l_{2}^{4}-4 l_{0}^{2} l_{2}^{6}
$$




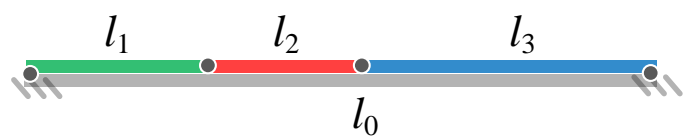

Figure 6.3: A shaky (immobile) configuration for 4-R linkage

$$
\begin{aligned}
& -4 l_{0}^{6} l_{3}^{2}+4 l_{0}^{4} l_{1}^{2} l_{3}^{2}+4 l_{0}^{2} l_{1}^{4} l_{3}^{2}+4 l_{0}^{4} l_{2}^{2} l_{3}^{2}-40 l_{0}^{2} l_{1}^{2} l_{2}^{2} l_{3}^{2}+4 l_{0}^{2} l_{2}^{4} l_{3}^{2}+6 l_{0}^{4} l_{3}^{4}+4 l_{0}^{2} l_{1}^{2} l_{3}^{4} \\
& +4 l_{0}^{2} l_{2}^{2} l_{3}^{4}-4 l_{0}^{2} l_{3}^{6}+l_{1}^{8}-4 l_{1}^{6} l_{2}^{2}+6 l_{1}^{4} l_{2}^{4}-4 l_{1}^{2} l_{2}^{6}+l_{2}^{8}-4 l_{1}^{6} l_{3}^{2}+4 l_{1}^{4} l_{2}^{2} l_{3}^{2}+4 l_{1}^{2} l_{2}^{4} l_{3}^{2} \\
& -4 l_{2}^{6} l_{3}^{2}+6 l_{1}^{4} l_{3}^{4}+4 l_{1}^{2} l_{2}^{2} l_{3}^{4}+6 l_{2}^{4} l_{3}^{4}-4 l_{1}^{2} l_{3}^{6}-4 l_{2}^{2} l_{3}^{6}+l_{3}^{8}=0
\end{aligned}
$$

which factorises as

$$
\begin{aligned}
& \left(l_{0}-l_{1}-l_{2}-l_{3}\right)\left(l_{0}+l_{1}-l_{2}-l_{3}\right)\left(l_{0}-l_{1}+l_{2}-l_{3}\right)\left(l_{0}+l_{1}+l_{2}-l_{3}\right) \\
& \quad\left(l_{0}-l_{1}-l_{2}+l_{3}\right)\left(l_{0}+l_{1}-l_{2}+l_{3}\right)\left(l_{0}-l_{1}+l_{2}+l_{3}\right)\left(l_{0}+l_{1}+l_{2}+l_{3}\right)=0
\end{aligned}
$$

or, simply, the product of the linear Grashof-type conditions. This union of eight hyperplanes has codimension 1 as an algebraic variety in the design space $\mathbb{R}^{4}$. Furthermore, the factorisation yields non-generic mechanisms that include some overconstrained cases. Consider the case $l_{0}-l_{1}-l_{2}-l_{3}=0$, for instance; it has a single shaky (infinitesimally mobile) configuration but is immobile, see Figure 6.3.

\subsubsection{Input mapping and singularities}

Classically, $L_{1}$ is considered to be the input link so $J_{1}$ is the actuated joint. Assuming $L_{0}$ is the base to which the reference frame $R$ is attached, we have $\theta_{0}=0$ in (4.31) and the joint variable $\phi_{1}=\theta_{1}$. This spans the A-space and the input map is the restriction to C-space of the projection

$$
\hat{\pi}_{a}\left(x_{i}, y_{i}, \theta_{i}\right)_{i=1,2,3}=\theta_{1}
$$

Following Theorem 5.8, taking derivatives of $\Pi_{a}:=\left(\hat{\pi}_{a}, F\right): \mathbb{R}^{9} \rightarrow \mathbb{R}^{8} \times \mathbb{R}$ results in the $(9 \times 9)$ Jacobian which can be reduced, for the purpose of calculating singularities, to a $(3 \times 3)$ matrix by row operations and deletions 


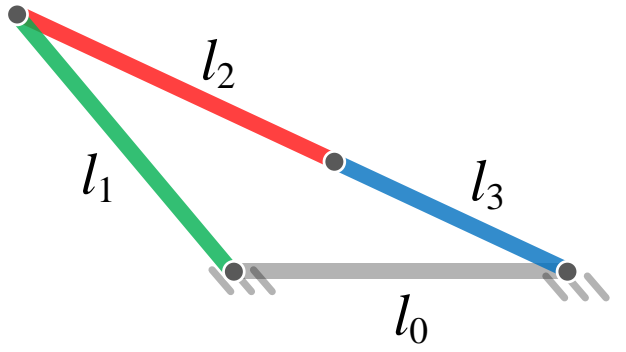

Figure 6.4: An input singularity for 4-R linkage

of rows and columns with leading $1 \mathrm{~s}$

$$
J \Pi_{a}^{\mathrm{red}}=\left[\begin{array}{ccc}
1 & 0 & 0 \\
-l_{1} s_{1} & -l_{2} s_{2} & -l_{3} s_{3} \\
l_{1} c_{1} & l_{2} c_{2} & l_{3} c_{3}
\end{array}\right]
$$

Its columns correspond to partial derivatives with respect to $\theta_{i}, i=1,2,3$. This matrix is rank deficient if the determinant of the bottom right $(2 \times 2)$ block matrix vanishes

$$
-l_{2} l_{3} s_{2,3}=0
$$

Suppose $l_{2}, l_{3} \neq 0$, then equation (6.13) holds if $\theta_{2}=\theta_{3} \bmod \pi$. As illustrated in Figure 6.4, this input singularity corresponds to a configuration where $L_{2}, L_{3}$ are aligned.

\subsubsection{Output mapping and singularities}

Following the classical consideration of mechanism theory, we first choose $L_{3}$ and then $L_{2}$ to be the output link. Since $L_{3}$ is connected to the base, only one pose variable (the orientation) is sufficient to specify the output map. However, if $L_{2}$ is the output, then at least two pose variables are needed to locate it in the plane. By these two different choices for the output link, we aim to show that other approaches, like that of Zlatanov et al. [2] or Gosselin and Angeles [1], are not capable of giving a full analysis (dimension-wise) if $L_{2}$ is considered as output. 


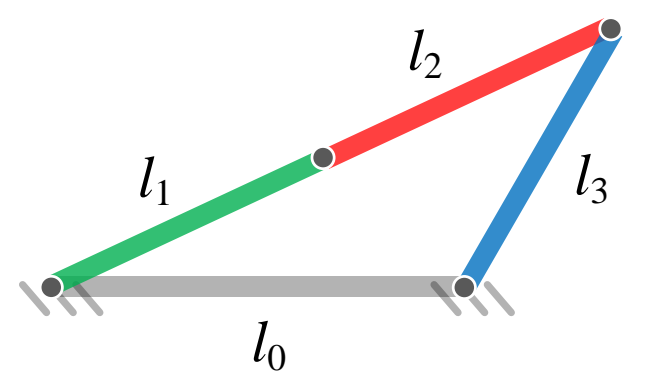

Figure 6.5: An output singularity corresponding to $L_{3}$ for 4-R linkage

If $L_{3}$ is the output link, then the $\mathrm{W}$-space can be parametrised by $\theta_{3} \in \mathbb{R}$ alone and $\operatorname{dim} \mathcal{W}=1$. The output map is then the restriction to the $\mathrm{C}$-space of the projection

$$
\hat{\pi}_{w}\left(x_{i}, y_{i}, \theta_{i}\right)_{i=1,2,3}=\theta_{3}
$$

In order to determine the output singularities, partial derivatives of $\Pi_{w}:=$ $\left(\hat{\pi}_{w}, F\right): \mathbb{R}^{9} \rightarrow \mathbb{R}^{8} \times \mathbb{R}$ are considered. After row operations and deletions of rows and columns with leading 1 s, the $(3 \times 3)$ reduced Jacobian matrix is obtained

$$
J \Pi_{w}^{\mathrm{red}}=\left[\begin{array}{ccc}
0 & 0 & 1 \\
-l_{1} s_{1} & -l_{2} s_{2} & -l_{3} s_{3} \\
l_{1} c_{1} & l_{2} c_{2} & l_{3} c_{3}
\end{array}\right]
$$

This matrix is rank deficient if determinant of the bottom left $(2 \times 2)$ block matrix vanishes

$$
-l_{1} l_{2} s_{1,2}=0
$$

Assume $l_{1}, l_{2} \neq 0$, equation (6.16) holds if $\theta_{1}=\theta_{2} \bmod \pi$. As shown in Figure 6.5, the output singularity corresponds to a configuration where $L_{1}, L_{2}$ are aligned. In this case, there is a "symmetry" between input and output maps which is absent in the next case.

If $L_{2}$ is considered as the output link, then no single pose variable sufficient to describes its displacement so $\theta_{2}, y_{2}$ are chosen to parametrise the $\mathrm{W}$-space. The output map associated to $L_{2}$ is the restriction to the $\mathrm{C}$-space 


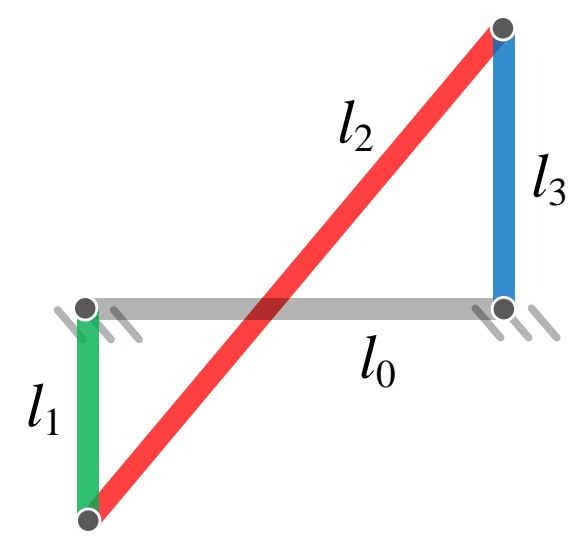

Figure 6.6: An output singularity corresponding to $L_{2}$ for 4-R linkage

of the projection

$$
\hat{\pi}_{w}\left(x_{i}, y_{i}, \theta_{i}\right)_{i=1,2,3}=\left[\theta_{2}, y_{2}\right]^{T}
$$

In order to determine the output singularities, partial derivatives of $\Pi_{w}:=$ $\left(\hat{\pi}_{w}, F\right): \mathbb{R}^{9} \rightarrow \mathbb{R}^{8} \times \mathbb{R}^{2}$ are considered. For the purpose of singularity analysis, the $(5 \times 4)$ reduced Jacobian matrix is obtained by row operations and deletions of rows and columns with leading 1 s

$$
J \Pi_{w}^{\mathrm{red}}=\left[\begin{array}{cccc}
0 & 0 & 1 & 0 \\
0 & 0 & 0 & 1 \\
l_{1} s_{1} & l_{3} s_{3} & 0 & l_{2} s_{2} \\
-l_{1} c_{1} & -l_{3} c_{3} & 0 & -l_{2} c_{2} \\
-l_{1} c_{1} & 0 & 1 & 0
\end{array}\right]
$$

Its columns correspond to partial derivatives with respect to $\theta_{1}, \theta_{3}, y_{2}, \theta_{2}$. This matrix is rank deficient if the $(2 \times 2)$ determinants of the bottom left $(3 \times 2)$ block matrix vanish simultaneously

$$
-l_{1} l_{3} s_{1,3}=l_{1} l_{3} c_{1} s_{3}=-l_{1} l_{3} c_{1} c_{3}=0
$$

Assume $l_{1}, l_{3} \neq 0$, these equations hold true if $\theta_{i}=\frac{\pi}{2}+\eta_{i} \pi, i=1,3$ for some integers $\eta_{i}$. As illustrated in Figure 6.6, the output singularity corresponds 


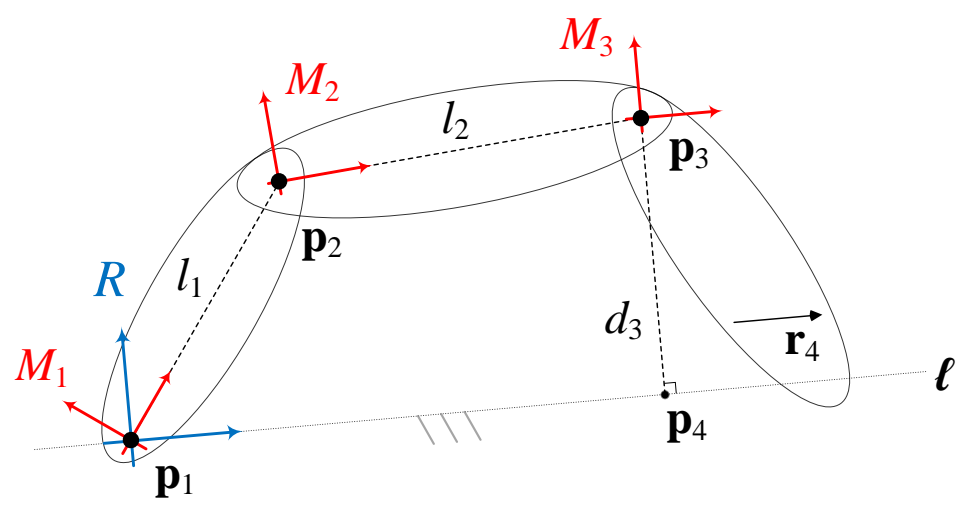

Figure 6.7: 3RP linkage with associated moving frames and design parameters

to a configuration where $L_{1}, L_{3}$ are parallel.

\subsection{Planar RRRP linkage}

To illustrate the model when a P-joint is involved, we adapt the previous example. This linkage consists of four rigid links connected by three R-joints and one P-joint, so that $k=t=4$ in the notation of Section 4.1. Assume $L_{0}$ connected by the P-joint is fixed. The reference frame $R$ and the moving frames $M_{i}, i=1,2,3$ are shown in Figure 6.7. The free displacement of each remaining link is represented by pose variables $\left(\theta_{i}, x_{i}, y_{i}\right)^{T}, i=1,2,3$.

As in Sections 4.3.1 and 4.3.2, the R-joint are geometrically modelled by points in each connected links and the P-joint by a directed line. Design parameters are lengths $l_{1}, l_{2}$ of the first and second links and the perpendicular distances $d_{3}$ in the third one. Symbolically, body and reference-frame coordinates of the three R-joints are in the form (6.1) and (6.2). They give rise to constraint equations of the form (4.12). On the other hand, body coordinates associated to the $\boldsymbol{\ell}$ representing the $\mathrm{P}$-joint with parametric form $\mathbf{p}+\lambda \mathbf{r}$ are

$$
\mathbf{p}_{1}^{R}=(0,0)^{T}, \quad \mathbf{p}_{4}^{3}=\left(0,-d_{3}\right)^{T}, \quad \mathbf{r}_{4}^{3}=\mathbf{r}_{4}^{R}=(1,0)^{T}
$$




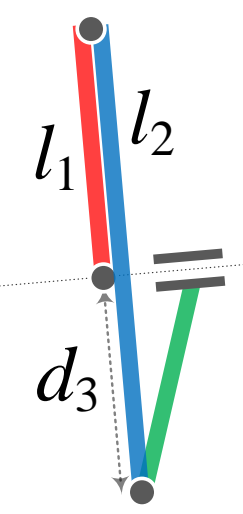

Figure 6.8: A C-space singularity for RRRP linkage

By using (2.6), reference-frame coordinates associated to the P-joint are determined

$$
\mathbf{P}_{4}^{3}=A_{3} \mathbf{p}_{4}^{3}+\mathbf{a}_{3}, \quad \mathbf{P}_{1}^{R}=A_{R} \mathbf{p}_{1}^{R}+\mathbf{a}_{R}, \quad \mathbf{R}_{4}^{3}=A_{3} \mathbf{r}_{4}^{3}, \quad \mathbf{R}_{4}^{R}=A_{R} \mathbf{r}_{4}^{R}
$$

which give rise to two equations of the form (4.15a) and (4.15b)

$$
\left(\mathbf{R}_{4}^{3} \times \mathbf{R}_{4}^{R}\right)_{z}=0, \quad\left(\left[\mathbf{P}_{4}^{3}-\mathbf{P}_{1}^{R}\right] \times \mathbf{R}_{4}^{R}\right)_{z}=0
$$

In terms of the given choice of coordinates, from (4.13) and (4.16), the eight constraint equations give rise to the $\mathrm{KCM} F: S E(2)^{3} \rightarrow \mathbb{R}^{8}$

$$
\begin{gathered}
\left(x_{i}, y_{i}, \theta_{i}\right)_{i=1,2,3} \mapsto\left[-x_{1},-y_{1}, x_{1}-x_{2}+l_{1} c_{1}, y_{1}-y_{2}+l_{1} s_{1}, x_{2}-x_{3}+l_{2} c_{2},\right. \\
\left.y_{2}-y_{3}+l_{2} s_{2},-s_{3}, s_{3} x_{3}-c_{3} y_{3}+d_{3}\right]^{T}
\end{gathered}
$$

\subsubsection{C-space singularities and Grashof-type conditions}

The positive corank condition on the resulting $(8 \times 9)$ Jacobian matrix can be reduced to considering the following $(2 \times 3)$ matrix by row operations and deletions of rows and columns with leading 1s 


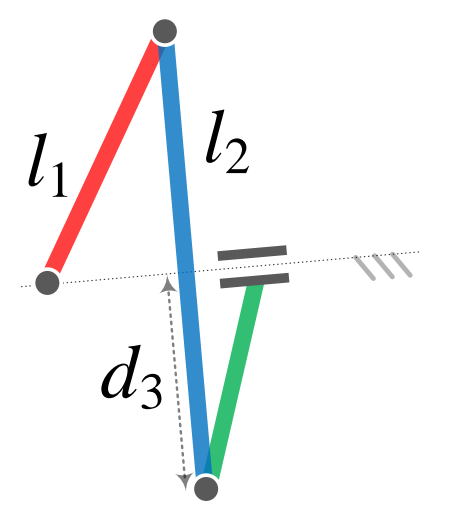

Figure 6.9: An input singularity for RRRP linkage

$$
J_{1} F^{\mathrm{red}}=\left[\begin{array}{ccc}
-l_{1} c_{1,3} & -l_{2} c_{2,3} & c_{3} x_{3}+s_{3} y_{3} \\
0 & 0 & -c_{3}
\end{array}\right]
$$

where $c_{i, 3}=\cos \left(\theta_{i}-\theta_{3}\right), i=1,2$.

The singular set $\Sigma_{1}^{1} F$ is defined by three $(2 \times 2)$ minors vanishing simultaneously and the equations yield

$$
\theta_{1}=\theta_{2}+\eta_{1} \pi, \quad \theta_{2}=\theta_{3}+\frac{\pi}{2}+\eta_{2} \pi \quad \eta_{1}, \eta_{2} \in \mathbb{Z}
$$

Geometrically, the three R-joints must be collinear and on a line perpendicular to the direction of the P-joint i.e. the first two links must be perpendicular to the translational axis of the P-joint.

Substituting (6.24) into (6.22) and eliminating $x_{i}, y_{i}, i=1,2,3$, we obtain Grashof-type conditions

$$
d_{3} \pm l_{1} \pm l_{2}=0
$$

A corresponding C-space singularity configuration is illustrated in Figure 6.8. This is the simplest case of the conditions found by Xue et al. [109] determining rotatability of components of closed chains with P-joints. 


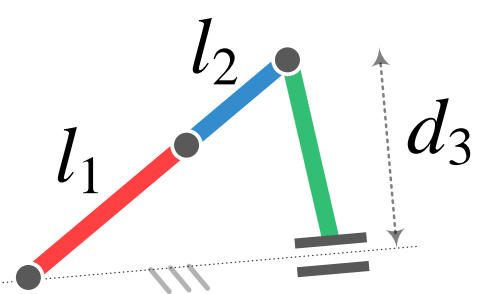

Figure 6.10: An output singularity corresponding to $L_{3}$ for RRRP linkage

\subsubsection{Input mapping and singularities}

As in Section 6.1.2, we choose $L_{1}$ as input so the actuated joint variable is $\phi_{1}=\theta_{1}$. Applying Theorem 5.8, the $(9 \times 9)$ Jacobian matrix can be reduced (by row operations and deletions of rows and columns with leading 1s) so that input singularites are determined by the $(3 \times 3)$ determinant

$$
\operatorname{det}\left[\begin{array}{ccc}
1 & 0 & 0 \\
-l_{1} c_{1,3} & -l_{2} c_{2,3} & c_{3} x_{3}+s_{3} y_{3} \\
0 & 0 & -c_{3}
\end{array}\right]=l_{2} c_{3} c_{2,3}=0
$$

Clearly from the KCM in (6.22), $s_{3}=0$ so $\theta_{3}=0 \bmod \pi$. Assuming $l_{2} \neq 0$, equation (6.26) holds true if $\theta_{2}=\theta_{3}+\pi / 2+\eta_{2} \pi$ for some integer $\eta_{2}$. This input singularity corresponds to a configuration where $L_{2}$ is perpendicular to the direction of the prismatic joint, as shown in Figure 6.9.

\subsubsection{Output mapping and singularities}

According to what is explained in Section 6.1.3, we are going to consider $L_{3}$ and $L_{2}$ to be output.

Suppose $L_{3}$ is output, then the $\mathrm{W}$-space is parametrised by $x_{3} \in \mathbb{R}$. So the output map is the restriction to the C-space of the projection

$$
\hat{\pi}_{w}\left(x_{i}, y_{i}, \theta_{i}\right)_{i=1,2,3}=x_{3}
$$

Then, output singularities can be determined by the reduced $(3 \times 3)$ deter- 


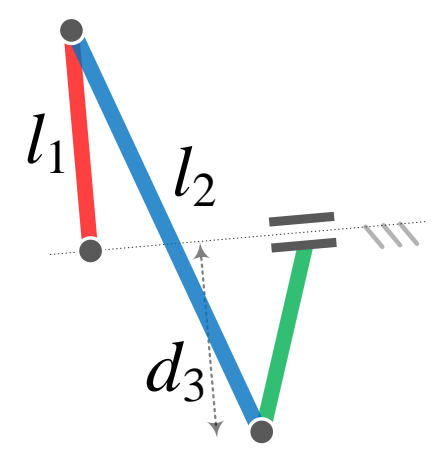

Figure 6.11: An output singularity corresponding to $L_{2}$ for RRRP linkage

minant

$$
\operatorname{det}\left[\begin{array}{ccc}
-l_{1} s_{1} & -l_{2} s_{2} & 0 \\
-l_{1} c_{1} c_{3} & -l_{2} c_{2} c_{3} & x_{3} c_{3}+y_{3} s_{3} \\
0 & 0 & -c_{3}
\end{array}\right]=-l_{1} l_{2} c_{3}^{2} s_{1,2}
$$

Assume $l_{1}, l_{2} \neq 0$, equation (6.28) equals zero if $\theta_{1}=\theta_{2} \bmod \pi$. As shown in Figure 6.10, the output singularity corresponds to a configuration where $L_{1}, L_{2}$ are aligned.

If $L_{2}$ is considered as output, then its displacement should be described by $\theta_{2}, y_{2}$. The output map associated to $L_{2}$ is the restriction to the C-space of the projection

$$
\hat{\pi}_{w}\left(x_{i}, y_{i}, \theta_{i}\right)_{i=1,2,3}=\left[\theta_{2}, y_{2}\right]^{T}
$$

In order to determine the output singularities, partial derivatives of $\Pi_{w}:=$ $\left(\hat{\pi}_{w}, F\right): \mathbb{R}^{9} \rightarrow \mathbb{R}^{8} \times \mathbb{R}^{2}$ are considered. After row operations and deletions of rows and columns with leading 1s, for the purpose of singularity analysis, the $(3 \times 2)$ reduced Jacobian matrix is considered

$$
J \Pi_{w}^{\mathrm{red}}=\left[\begin{array}{cc}
l_{1} c_{1} & 0 \\
-l_{1} s_{1} s_{3} & x_{3} c_{3}+y_{3} s_{3} \\
0 & -c_{3}
\end{array}\right]
$$




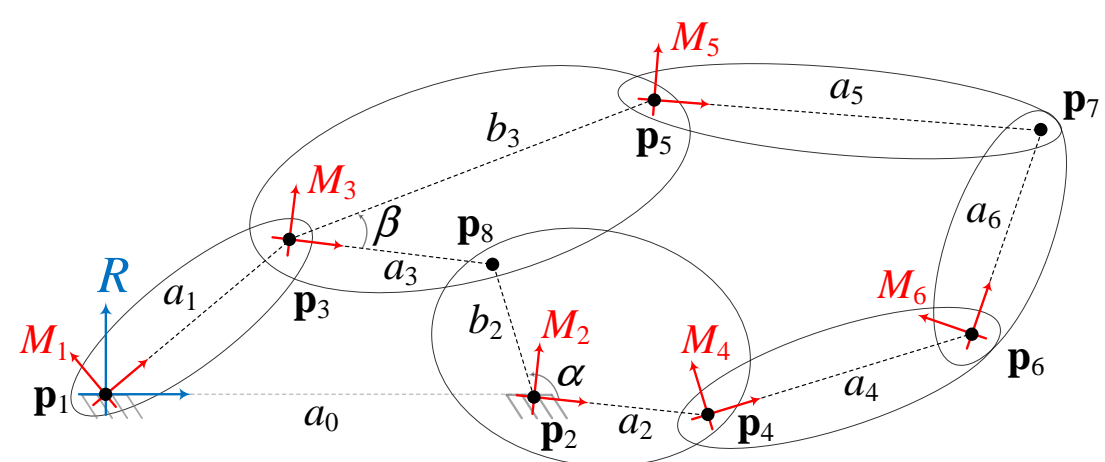

Figure 6.12: Extended Watt 7-R linkage with associated moving frames and design parameters

This matrix is rank deficient if its three $(2 \times 2)$ minors vanish simultaneously

$$
-l_{1} c_{1} c_{3}=l_{1} c_{3} s_{1} s_{3}=l_{1} c_{1}\left(x_{3} c_{3}+y_{3} s_{3}\right)=0
$$

Assume $l_{1}>0$, these equations hold true if $\theta_{1}=\frac{\pi}{2}+\eta \pi$ for some integers $\eta$. Note that $\theta_{3}=0 \bmod \pi$ from the KCM in (6.22). As illustrated in Figure 6.11, the output singularity corresponds to a configuration where $L_{1}$ is perpendicular to the direction of the prismatic joint.

\subsection{Extended Watt planar linkage}

To complete the examples of classical planar linkages, the final case has two degrees of freedom and in some respects is a counterexample to universal statements about genericity.

As shown in Figure 6.12, this mechanism consists of six mobile links together with a fixed base, which can be considered as a combination of planar 4 -R and planar $5 \mathrm{R}$ mechanisms that share common links $L_{2}, L_{3}$. The frames $M_{i}, i=1, \ldots 6$ are assigned to the moving bodies whose poses are described by $\left(\theta_{i}, x_{i}, y_{i}\right), i=1, \ldots, 6$ measured in the reference frame $R$ attached to fixed link $L_{0}$. There are 11 design parameters $a_{i}, i=0, \ldots, 6, b_{2}, b_{3}, \alpha, \beta$. It is assumed that all $a_{i}, b_{i}>0$. 
The eight R-joints each imposes two constraint equations, so the $\mathrm{KCM}$ has the form $F: S E(2)^{6} \rightarrow \mathbb{R}^{16}$ given by

$$
\begin{aligned}
\left(x_{i}, y_{i}, \theta_{i}\right)_{i=1, \ldots, 6} \mapsto & {\left[x_{1}, y_{1},-a_{0}+x_{2}, y_{2},-x_{1}+x_{3}-a_{1} c_{1},-y_{1}+y_{3}-a_{1} s_{1}\right.} \\
& -x_{2}+x_{4}-a_{2} c_{2},-y_{2}+y_{4}-a_{2} s_{2},-x_{3}+x_{5}-b_{3} c_{\beta, 3}, \\
& -y_{3}+y_{5}-b_{3} s_{\beta, 3},-x_{4}+x_{6}-a_{4} c_{4},-y_{4}+y_{6}-a_{4} s_{4}, \\
& -x_{5}+x_{6}-a_{5} c_{5}+a_{6} c_{6},-y_{5}+y_{6}-a_{5} s_{5}+a_{6} s_{6}, \\
& \left.-x_{2}+x_{3}-b_{2} c_{\alpha, 2}+a_{3} c_{3},-y_{2}+y_{3}-b_{2} s_{\alpha, 2}+a_{3} s_{3}\right]^{T}
\end{aligned}
$$

Here $c_{\alpha, 2}=\cos \left(\alpha+\theta_{2}\right), c_{\beta, 3}=\cos \left(\beta+\theta_{3}\right)$ and similarly for sines. For a fixed choice of design parameters, we would anticipate the C-space to have dimension 2, corresponding to its mobility.

In order to determine whether this family of mechanisms is generic in the sense that its C-space is a 2-dimensional manifold for almost all choices of design parameters, it is necessary to check whether the $(16 \times 29)$ Jacobian matrix of $\hat{F}$ (with respect to all 18 pose variables and 11 design parameters) has full rank. The Jacobian can be reduced to a $(3 \times 16)$ matrix

$$
\begin{aligned}
J \hat{F}^{\mathrm{red}}= & {\left[\begin{array}{ccccccccc}
a_{1} c_{1} & -a_{2} c_{2} & & b_{3} c_{\beta, 3} & -a_{4} c_{4} & a_{5} c_{5} & -a_{6} c_{6} & s_{1} \\
0 & a_{2} s_{2}-b_{2} s_{\alpha, 2} & a_{3} s_{3}-b_{3} s_{\beta, 3} & a_{4} s_{4} & -a_{5} s_{5} & a_{6} s_{6} & 0 \\
-a_{1} c_{1} & b_{2} c_{\alpha, 2} & & -a_{3} c_{3} & 0 & 0 & 0 & -s_{1} \\
s_{\beta, 3} & b_{3} c_{\beta, 3} & 0 & 0 & -s_{2} & -s_{4} & s_{5} & 0 & -s_{6} \\
c_{\beta, 3} & -b_{3} s_{\beta, 3} & c_{\alpha, 2} & -b_{2} s_{\alpha, 2} & -c_{2} & -c_{4} & c_{5} & -c_{3} & -c_{6} \\
0 & 0 & s_{\alpha, 2} & b_{2} c_{\alpha, 2} & 0 & 0 & 0 & -s_{3} & 0
\end{array}\right] }
\end{aligned}
$$

Given the assumptions on design parameters, this matrix is never rank deficient so that $\hat{F} \pitchfork\{\mathbf{0}\}$ and the D-space is a submanifold of codimension 16 . We can conclude that for almost all choices of design parameters, the C-space is a manifold (non-singular).

Turning to the C-space singularities and possible Grashof-type conditions, the rank of the $(16 \times 18)$ Jacobian matrix $J_{1} F$ is the same as the following reduced $(4 \times 6)$ matrix which can be obtained after row operations and dele- 


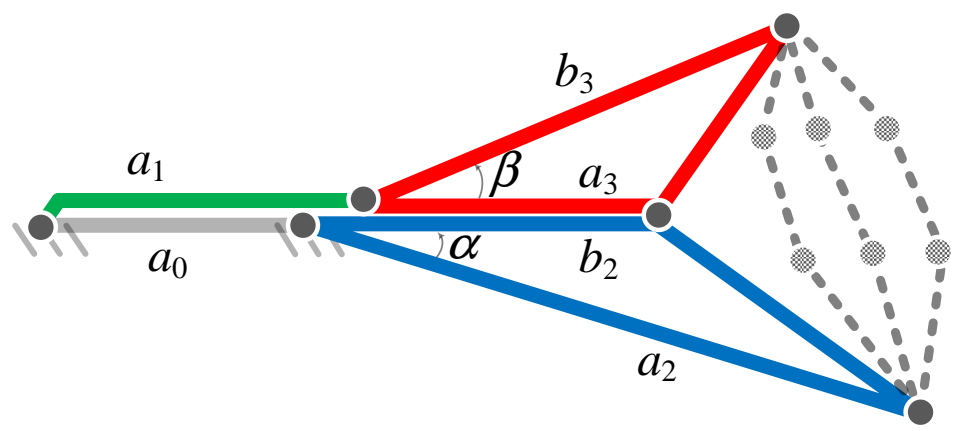

Figure 6.13: A C-space singularity associated to the 4-R at a flat configuration

tions of rows and columns with leading 1s

$$
J_{1} F^{\mathrm{red}}=\left[\begin{array}{cccccc}
a_{1} s_{1} & -a_{2} s_{2} & b_{3} s_{\beta, 3} & -a_{4} s_{4} & a_{5} s_{5} & -a_{6} s_{6} \\
-a_{1} c_{1} & a_{2} c_{2} & -b_{3} c_{\beta, 3} & a_{4} c_{4} & -a_{5} c_{5} & a_{6} c_{6} \\
-a_{1} s_{1} & b_{2} s_{\alpha, 2} & -a_{3} s_{3} & 0 & 0 & 0 \\
a_{1} c_{1} & -b_{2} c_{\alpha, 2} & a_{3} c_{3} & 0 & 0 & 0
\end{array}\right]
$$

Of the $15(4 \times 4)$ minors, 12 are non-trivial and can be solved simultaneously using Mathematica (see [110]) to give distinct solutions determining the set $\Sigma_{1}^{1} F$. From (5.7), it is expected that this set has codimension 3. However, one of the possible solutions is given by

$$
\theta_{2}=\theta_{1}-\alpha+\eta_{2} \pi, \quad \theta_{3}=\theta_{1}+\eta_{3} \pi \quad \eta_{2}, \eta_{3} \in \mathbb{Z}
$$

defining submanifolds of codimension 2 in the space of pose variables. Although there are other singular branches with various codimensions, this solution set relates simply to the base 4-R linkage visible in Figure 6.12. This tells us that there is a branch of singularities along which the Jacobian $J_{1} F$ is not transverse to $\Sigma^{1}$. As illustrated in Figure 6.13, nevertheless, since the conditions only relate to the 4 -R, we may still obtain the Grashof-type conditions

$$
a_{0} \pm a_{1} \pm b_{2} \pm a_{3}=0
$$




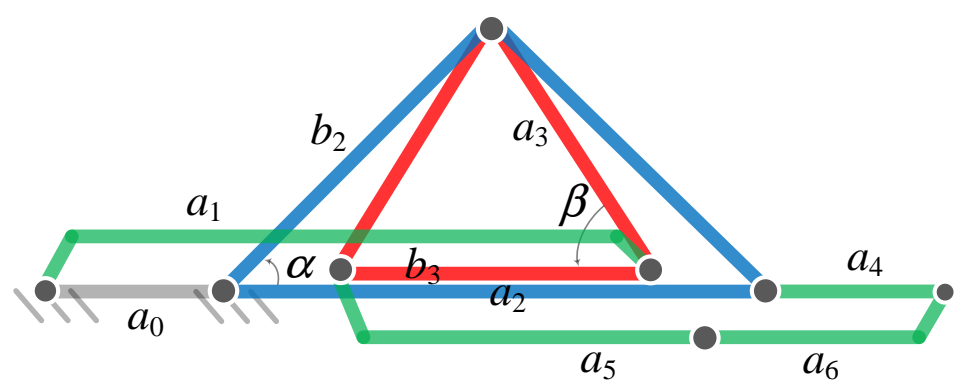

Figure 6.14: A flat configuration corresponding to the C-space singularity

As mentioned, there are other singular branches among which the most relevant one is of the form

$$
\theta_{1}=h\left(\theta_{2}, \theta_{3}, \theta_{4}\right), \quad \theta_{5}=\theta_{4}+\eta_{5} \pi, \quad \theta_{6}=\theta_{4}+\eta_{6} \pi \quad \eta_{5}, \eta_{6} \in \mathbb{Z}
$$

where $h$ is a specific function. Given this branch has the correct codimension, it is likely that it will give rise to corresponding Grashof-type conditions but the form of this has not yet been determined. One special case of (6.37) is given by adding conditions on the remaining pose variables

$$
\theta_{i}=\theta_{1}+\eta_{i} \pi, i \in\{2,4,5,6\}, \quad \theta_{3}=\theta_{1}-\beta+\eta_{3} \pi \quad \eta_{i}, \eta_{3} \in \mathbb{Z}
$$

These correspond to the configuration where all the mobile links (i.e. all the $x$-axes of the moving frames) are parallel. Eliminating pose variables leads to a collection of Grashof-type conditions on the design parameters as follows

$$
\begin{aligned}
a_{0} \pm a_{1} \pm a_{2} \pm a_{4} \pm a_{5} \pm a_{6} \pm b_{3} & =0 \\
a_{0} \pm a_{1} \pm b_{2} c_{\alpha} \pm a_{3} c_{\beta} & =0 \\
b_{2} s_{\alpha} \pm a_{3} s_{\beta} & =0
\end{aligned}
$$

This flat configuration is illustrated in Figure 6.14. 


\section{Chapter 7}

\section{Planar parallel manipulators}

In this Chapter, the constraint-based kinematic modelling approach is applied to two extensively studied planar parallel manipulator (PPM) architectures. These are in the standard form for providing full mobility for a platform. The platform is connected by three serial legs to the base and each leg has three 1-dof joints. The CGK formula readily demonstrates that, in the general case, the PPM has mobility $\mu=3$. In practical applications, one joint in each leg is actuated.

The constraint-based approach provides what appears to be a Grashoftype condition that is new. It also demonstrates the effect of many different choices of actuator.

\subsection{Planar 3-RRR parallel manipulator}

The 3-RRR has an extensive structure on its kinematics and singularities [111, 28]. We work in complete generality so that the three base and three platform anchor points may have any layout. This includes the possibility that they may coincide or be collinear. The variations are captured by design parameters.

The 3-RRR PPM is illustrated in Figure 7.1, which shows a choice of 


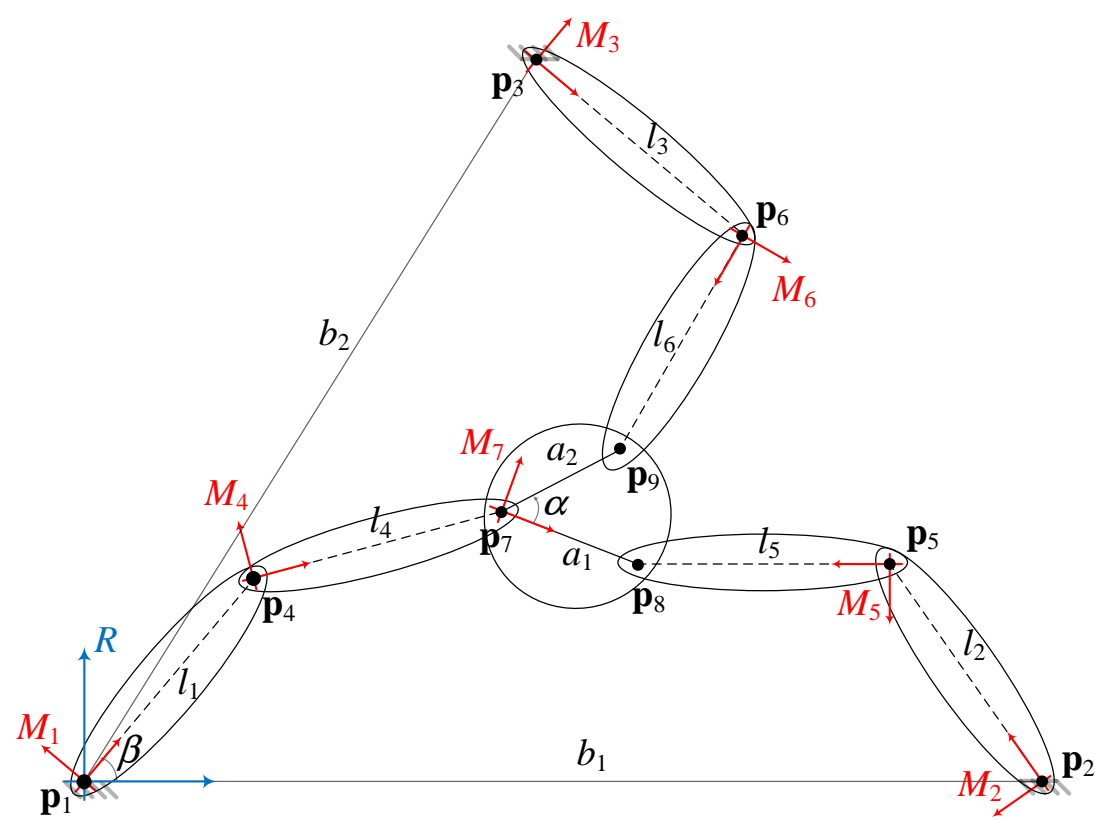

Figure 7.1: 3-RRR PPM with associated moving frames and design parameters

moving and reference frames together with design parameters. The mechanism consists of $k=8$ rigid bodies where we regard the ambient space as the fixed base. Therefore we require 21 pose variables and there are 12 design parameters

- design parameters of the links (link lengths): $l_{i}, i=1, \ldots, 6$

- design parameters of the platform: $a_{1}, a_{2}, \alpha$

- design parameters of the base: $b_{1}, b_{2}, \beta$

With the chosen reference and moving frames, the body coordinates of each of the nine R-joints in 'preceding' and 'following' frames are

$$
\begin{array}{lll}
\mathbf{p}_{i}^{-}=\left(l_{i-3}, 0\right)^{T}, & \mathbf{p}_{j}^{+}=(0,0)^{T}, & i=4, \ldots, 9 \quad j=1, \ldots, 7 \\
\mathbf{p}_{1}^{R}=(0,0)^{T}, & \mathbf{p}_{2}^{R}=\left(b_{1}, 0\right)^{T}, & \mathbf{p}_{3}^{R}=\left(b_{2} c_{\beta}, b_{2} s_{\beta}\right)^{T}, \\
\mathbf{p}_{8}^{7}=\left(a_{1}, 0\right)^{T}, & \mathbf{p}_{9}^{7}=\left(a_{2} c_{\alpha}, a_{2} s_{\alpha}\right)^{T} &
\end{array}
$$

To find the reference-frame coordinates of each point in (7.1), we can follow the same procedure as in (6.2). Each R-joint imposes a pair of constraint 
equations of the form (4.12), so the $\mathrm{KCM}$ has the form $F: S E(2)^{7} \rightarrow \mathbb{R}^{18}$ given by

$$
\begin{aligned}
\left(x_{i}, y_{i}, \theta_{i}\right)_{i=1, \ldots, 7} \mapsto & {\left[x_{1}, y_{1}, x_{2}-b_{1}, y_{2}, x_{3}-b_{2} c_{\beta}, y_{3}-b_{2} s_{\beta},-x_{1}+x_{4}-l_{1} s_{1}\right.} \\
& -y_{1}+y_{4}-l_{1} s_{1},-x_{2}+x_{5}-l_{2} c_{2},-y_{2}+y_{5}-l_{2} s_{2} \\
& -x_{3}+x_{6}-l_{3} c_{3},-y_{3}+y_{6}-l_{3} s_{3},-x_{4}+x_{7}-l_{4} c_{4} \\
& -y_{4}+y_{7}-l_{4} s_{4},-x_{5}+x_{7}-l_{5} c_{5}+a_{1} c_{7} \\
& -y_{5}+y_{7}-l_{5} s_{5}+a_{1} s_{7},-x_{6}+x_{7}-l_{6} c_{6}+a_{2} c_{\alpha, 7} \\
& \left.-y_{6}+y_{7}-l_{6} s_{6}+a_{2} s_{\alpha, 7}\right]^{T}
\end{aligned}
$$

with the usual terminology for cosines and sines.

\subsubsection{C-space singularities and Grashof-type conditions}

The associated Jacobian matrix is $(18 \times 21)$. By row operations and deletions of rows and columns with leading 1s, the problem of determining rank deficiency can be reduced to considering the following $(4 \times 7)$ matrix

$$
\left[\begin{array}{ccccccc}
-l_{1} s_{1} & l_{2} s_{2} & 0 & -l_{4} s_{4} & l_{5} s_{5} & 0 & -a_{1} s_{7} \\
l_{1} c_{1} & -l_{2} c_{2} & 0 & l_{4} c_{4} & -l_{5} c_{5} & 0 & a_{1} c_{7} \\
0 & -l_{2} s_{2} & l_{3} s_{3} & 0 & -l_{5} s_{5} & l_{6} s_{6} & a_{1} s_{7}-a_{2} s_{\alpha, 7} \\
0 & l_{2} c_{2} & -l_{3} c_{3} & 0 & l_{5} c_{5} & -l_{6} c_{6} & -a_{1} c_{7}+a_{2} c_{\alpha, 7}
\end{array}\right]
$$

Its columns correspond to partial derivatives with respect to $\theta_{i}, i=1, \ldots, 7$. To determine $\Sigma_{1}^{1} F$ we must solve simultaneously the vanishing of $35(4 \times$ 4) minors (see Appendix B for part of the calculation). From (5.7), the corank 1 singularities are expected to have codimension 4, in other words, four equations are required to determine singularity. Many of the minors factorise and it becomes relatively straightforward to deduce that there are three possible conditions that correspond to collinearity of three closed 6$\mathrm{R}$ chains within the mechanism and one further condition corresponding to 


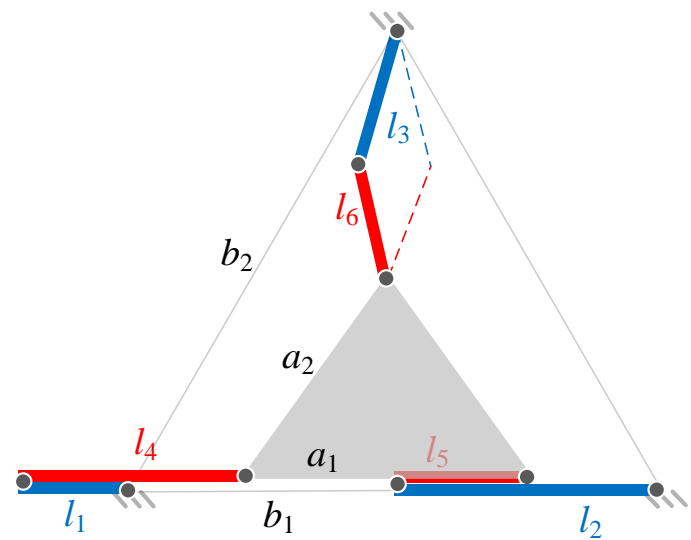

(a) Collinearity of bottom closed 6-R chain

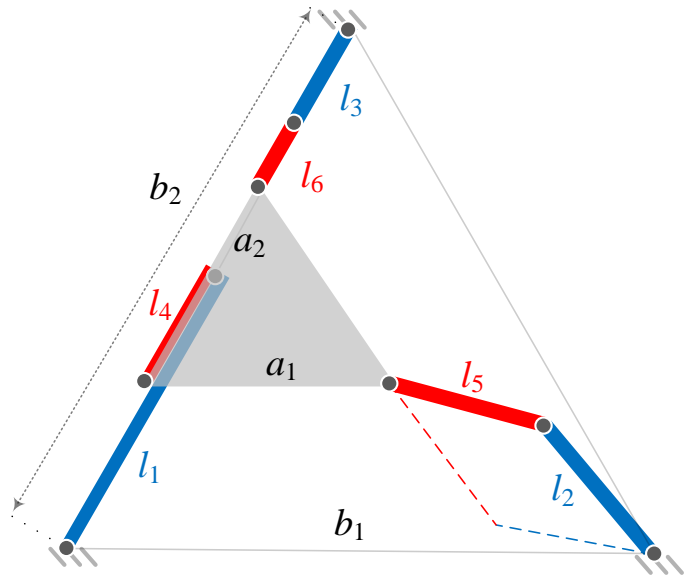

(b) Collinearity of left closed 6-R chain

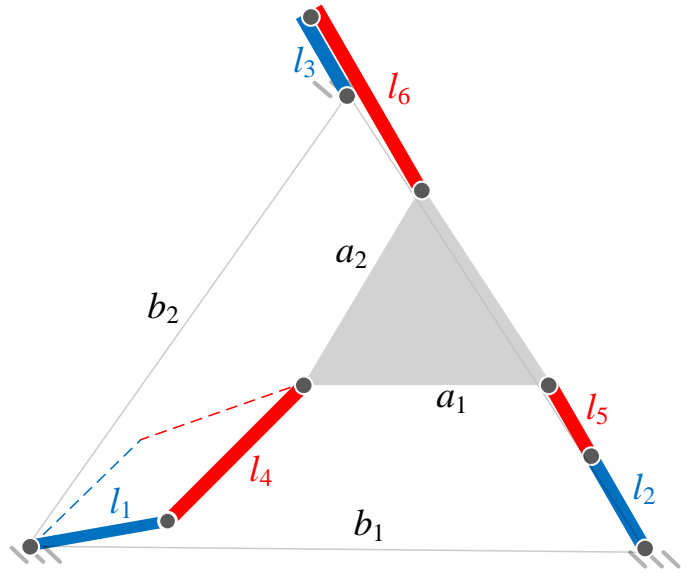

(c) Collinearity of right closed 6-R chain

Figure 7.2: C-space singularities for 3-RRR PM 
collinearity of the six leg links

$$
\begin{aligned}
\theta_{i}=\theta_{j}+\eta_{i j} \pi & i, j \in\{1,2,4,5,7\} \\
\theta_{i}=\theta_{j}+\delta_{j 7} \alpha+\eta_{i j} \pi & i, j \in\{1,3,4,6,7\} \\
\theta_{i}=\theta_{j}+\delta_{j 7}(\pi-\gamma)+\eta_{i j} \pi & i, j \in\{2,3,5,6,7\} \\
\theta_{i}=\theta_{j}+\eta_{i j} \pi & i, j \in\{1,2,3,4,5,6\}
\end{aligned}
$$

Here $\eta_{i j} \in \mathbb{Z}$ and $\delta_{j 7}$ is zero unless $j=7$ when it is 1 ; the corresponding angles, $\theta_{7}+\alpha$ and $\theta_{7}+\pi-\gamma$ in (7.4b) and (7.4c) are the angles the platform makes with the corresponding line joining the base anchor points in the closed chain. Note that the first three conditions are expressed by four equations but the fourth requires five equations and therefore imposes additional conditions on design parameters.

Substituting the singularity conditions into the KCM and setting equal to zero determines Grashof-type conditions necessary for C-space singularities. Equation (7.4a) fairly easily leads to

$$
b_{1} \pm a_{1} \pm l_{1} \pm l_{4} \pm l_{2} \pm l_{5}=0
$$

Geometrically, the bottom closed 6-R chain folds into a flattened hexagon. This is illustrated in Figure 7.2a. It is possible to make similar deductions for the following two singularity sets in equations (7.4b) and (7.4c), providing two different Grashof-type conditions in the form of (7.5) for the other closed chains.

However, an argument by symmetry, via an appropriate relabelling of the base and platform design parameters leads more simply to the same conclusion for the other closed chains, as is shown in Figures 7.2b and 7.2c. The diagram also shows that in a singular configuration there are potentially four combinations (see Appendix B for more details) of pose variables for the remaining 2-link leg though in practice not all of these may be feasible. 

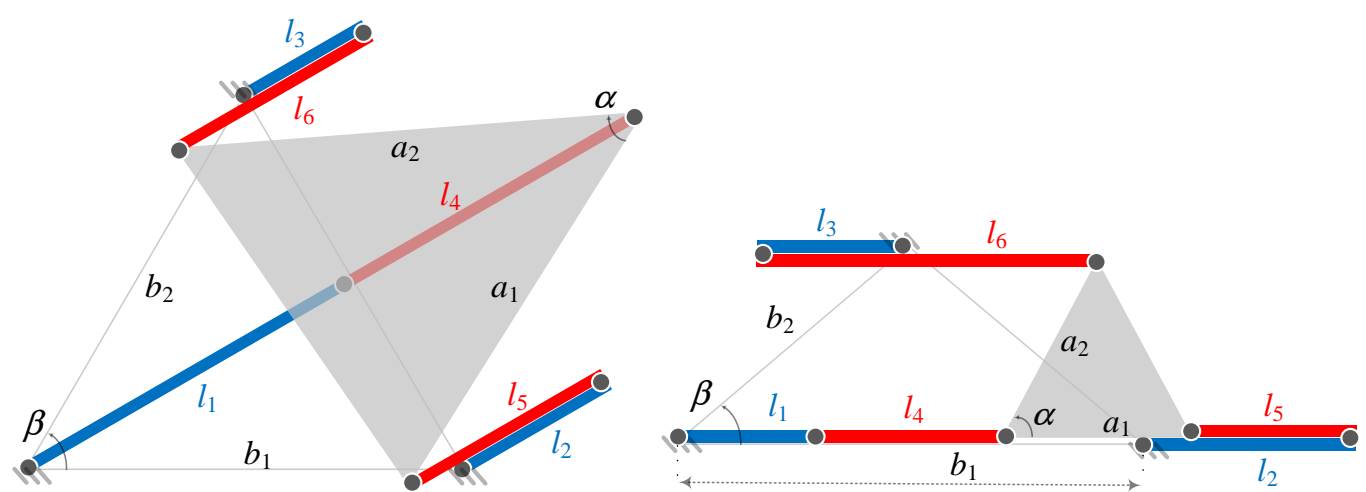

Figure 7.3: Special C-space singularities for 3-RRR PPM

The final singularity condition in (7.4d), when combined with the KCM, can be shown to give the following pair of Grashof-type conditions

$$
\begin{aligned}
b_{1}^{2} \vartheta^{2}+b_{2}^{2} \zeta^{2}- & 2 b_{1} b_{2} \zeta \vartheta \cos \beta \\
= & a_{1}^{2} \vartheta^{2}+a_{2}^{2} \zeta^{2}-2 a_{1} a_{2} \zeta \vartheta \cos \alpha \\
a_{1}^{2} b_{1}^{2}+a_{2}^{2} b_{2}^{2}-2 & a_{1} a_{2} b_{1} b_{2} \cos (\beta-\alpha) \\
= & a_{1}^{2} \vartheta^{2}+a_{2}^{2} \zeta^{2}-2 a_{1} a_{2} \zeta \vartheta \cos \alpha
\end{aligned}
$$

where $\zeta= \pm l_{1} \pm l_{4} \pm l_{2} \pm l_{5}$ and $\vartheta= \pm l_{1} \pm l_{4} \pm l_{3} \pm l_{6}$. These special cases are illustrated in Figure 7.3 (see Appendix B for a summary of this analysis).

\subsubsection{Output mapping and singularities}

Although each moving component could be considered as the end-effector, the central link or platform, as shown in Figure 7.1, is naturally chosen as the endeffector so that the workspace $\mathcal{W}$ is parametrised by $\left(x_{7}, y_{7}, \theta_{7}\right) \in \mathbb{R}^{3}$. The $\mathrm{KCM} F$ is given in (7.2). Let $\hat{\pi}_{w}: \mathbb{R}^{21} \rightarrow \mathcal{W}$ denote projection onto the $\mathrm{W}$ space, so that $\pi_{w}$ is the restriction of $\hat{\pi}_{w}$ to $\mathcal{C}$. To find the output singularities of 3-RRR, we apply Theorem 5.8 , so consider $\Pi_{w}:=\left(\hat{\pi}_{w}, F\right): \mathbb{R}^{21} \rightarrow \mathbb{R}^{18} \times \mathbb{R}^{3}$

$$
\left(x_{i}, y_{i}, \theta_{i}\right)_{i=1, \ldots, 7} \mapsto\left[x_{7}, y_{7}, \theta_{7}, F\left(x_{1}, \ldots, \theta_{7}\right)\right]^{T}
$$




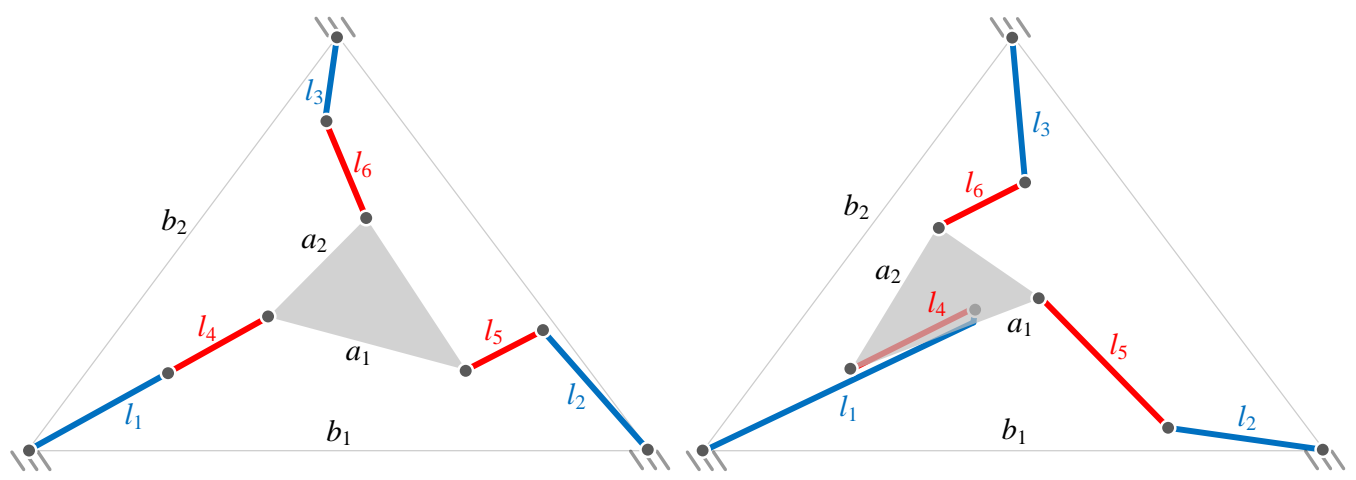

Figure 7.4: 3-RRR PPM output singularities

The $(21 \times 21)$ Jacobian matrix can be reduced by deleting rows and columns of leading 1 s in reduced-row echelon form to a $(9 \times 9)$ matrix

$$
J \Pi_{w}^{\mathrm{red}}=\left[\begin{array}{ccccccccc}
0 & 0 & 0 & 0 & 0 & 0 & 0 & 0 & 1 \\
0 & 0 & 0 & 0 & 0 & 0 & 0 & 1 & 0 \\
0 & 0 & 0 & 0 & 0 & 0 & 1 & 0 & 0 \\
-l_{1} s_{1} & 0 & 0 & -l_{4} s_{4} & 0 & 0 & 0 & 0 & -1 \\
l_{1} c_{1} & 0 & 0 & l_{4} c_{4} & 0 & 0 & 0 & -1 & 0 \\
0 & l_{2} s_{2} & 0 & 0 & l_{5} s_{5} & 0 & -a_{1} s_{7} & 0 & -1 \\
0 & -l_{2} c_{2} & 0 & 0 & -l_{5} c_{5} & 0 & a_{1} c_{7} & -1 & 0 \\
0 & 0 & l_{3} s_{3} & 0 & 0 & l_{6} s_{6} & -a_{2} s_{\alpha, 7} & 0 & -1 \\
0 & 0 & -l_{3} c_{3} & 0 & 0 & -l_{6} c_{6} & a_{2} c_{\alpha, 7} & -1 & 0
\end{array}\right]
$$

Its columns correspond to partial derivatives with respect to $\theta_{1}, \ldots, \theta_{7}, y_{7}, x_{7}$, and the top right $(3 \times 3)$ block matrix corresponds to the non-singular Jacobian of $\pi_{w}$. The bottom left $(6 \times 6)$ block matrix may be rank deficient if its determinant

$$
l_{1} l_{2} l_{3} l_{4} l_{5} l_{6} \sin \left(\theta_{1}-\theta_{4}\right) \sin \left(\theta_{2}-\theta_{5}\right) \sin \left(\theta_{3}-\theta_{6}\right)=0
$$

Assuming design parameters $l_{i} \neq 0, i=1, \ldots, 6$, equation (7.9) holds if $\theta_{i}=$ $\theta_{i+3}+\eta_{i} \pi, i=1,2,3$, for some integers $\eta_{i}$, corresponding to a configuration in which one or more pair of leg links is collinear, as illustrated in Figure 7.4 for the pair $l_{1}, l_{4}$. 

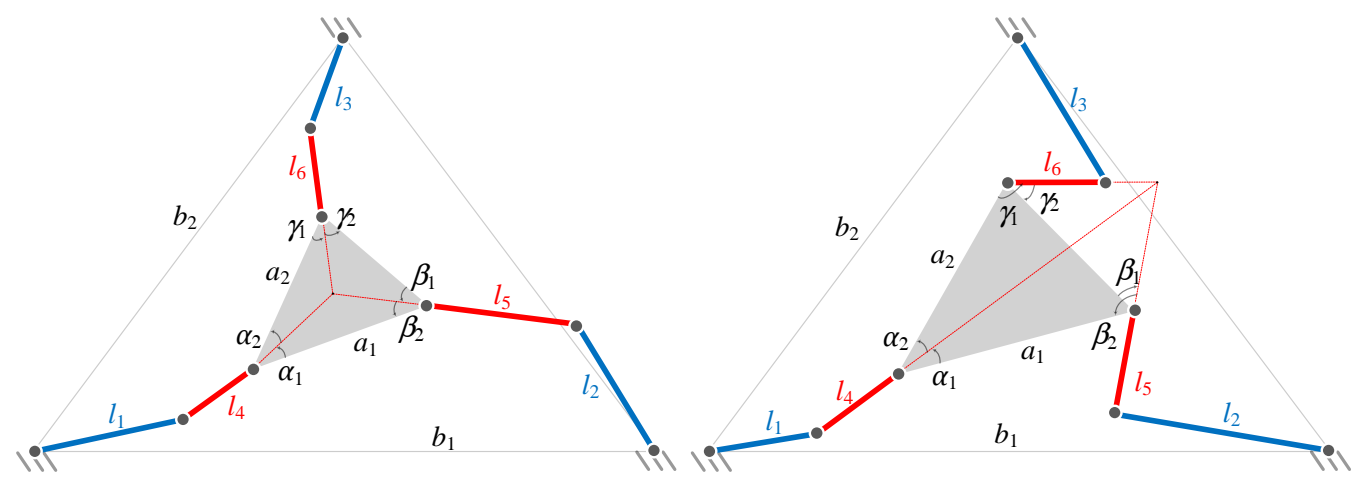

Figure 7.5: 3-RRR PPM input singularities

\subsubsection{Input mapping and singularities}

In this section, we will discuss two different possibilities as actuated joints [11, 28]. First, we assume actuators are placed at the base R-joints $\mathbf{p}_{1}, \mathbf{p}_{2}, \mathbf{p}_{3}$. Next, we consider the medial R-joints $\mathbf{p}_{4}, \mathbf{p}_{5}, \mathbf{p}_{6}$ to be actuated.

\section{3-RRR}

The three base joints $\mathbf{p}_{1}, \mathbf{p}_{2}, \mathbf{p}_{3}$ are the actuators. Identifying the base frame with the reference frame sets $\theta_{i^{-}}=0, i=1,2,3$ in (4.31). The joint variables are then given by $\phi_{i}=\theta_{i}, i=1,2,3$ which span the A-space and the input map is the restriction to $\mathcal{C}$ of the projection

$$
\hat{\pi}_{a}\left(x_{i}, y_{i}, \theta_{i}\right)_{i=1, \ldots, 7}=\left[\theta_{1}, \theta_{2}, \theta_{3}\right]^{T}
$$

Taking partial derivatives of $\Pi_{a}:=\left(\hat{\pi}_{a}, F\right): \mathbb{R}^{21} \rightarrow \mathbb{R}^{18} \times \mathbb{R}^{3}$ yields its $(21 \times 21)$ Jacobian matrix which can be reduced to a $(9 \times 9)$ matrix by row 
operations and deletions of rows and columns with leading 1s

$$
J_{a}^{\mathrm{red}}=\left[\begin{array}{ccccccccc}
1 & 0 & 0 & 0 & 0 & 0 & 0 & 0 & 0 \\
0 & 1 & 0 & 0 & 0 & 0 & 0 & 0 & 0 \\
0 & 0 & 1 & 0 & 0 & 0 & 0 & 0 & 0 \\
-l_{1} s_{1} & 0 & 0 & -l_{4} s_{4} & 0 & 0 & 0 & 0 & -1 \\
l_{1} c_{1} & 0 & 0 & l_{4} c_{4} & 0 & 0 & 0 & -1 & 0 \\
0 & -l_{2} s_{2} & 0 & 0 & -l_{5} s_{5} & 0 & a_{1} s_{7} & 0 & -1 \\
0 & l_{2} c_{2} & 0 & 0 & l_{5} c_{5} & 0 & -a_{1} c_{7} & -1 & 0 \\
0 & 0 & -l_{3} s_{3} & 0 & 0 & -l_{6} s_{6} & a_{2} s_{\alpha, 7} & 0 & -1 \\
0 & 0 & l_{3} c_{3} & 0 & 0 & l_{6} c_{6} & -a_{2} c_{\alpha, 7} & -1 & 0
\end{array}\right]
$$

In a similar manner to the output map, the input singularities are given by the determinant of the bottom right $(6 \times 6)$ submatrix of the reduced Jacobian

$$
-l_{4} l_{5} l_{6}\left(a_{1} \sin \left(\theta_{4}-\theta_{6}\right) \sin \left(\theta_{5}-\theta_{7}\right)+a_{2} \sin \left(\theta_{4}-\theta_{5}\right) \sin \left(\alpha-\theta_{6}+\theta_{7}\right)\right)=0
$$

Given $l_{i} \neq 0, i=4,5,6$, we employ a variant of Ceva's Theorem, given in (5.13), to interpret equation (7.12) geometrically. Setting $\theta_{4}=\psi_{a}, \theta_{5}=\psi_{b}$, $\theta_{6}=\psi_{c}$ and $\theta_{7}=\psi_{t}$, shows that this is equivalent to the lines spanned by links $L_{4}, L_{5}, L_{6}$ being concurrent. This input singularity is independent of platform orientation $\theta_{7}$. The intersection may take place either internally or externally to the platform triangle, as in Figure 7.5. Special cases include when two inner links and a side of the platform are collinear so one of the following sets of equations holds

$$
\begin{array}{rr}
\theta_{4}=\theta_{5}=\theta_{7} \quad \bmod \pi \\
\theta_{4}=\theta_{6}=\alpha+\theta_{7} \quad \bmod \pi \\
\theta_{5}=\theta_{6}-\alpha=\theta_{7} \quad \bmod \pi
\end{array}
$$

A final special case is when $\theta_{4}=\theta_{5}=\theta_{6} \bmod \pi$. This input singularity 


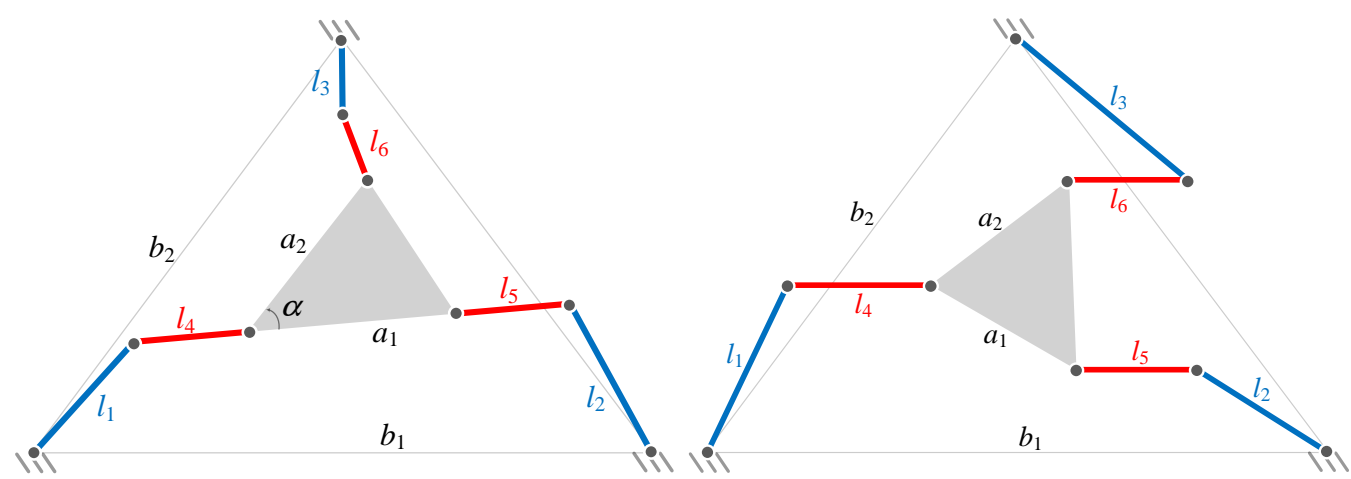

Figure 7.6: Special input singularities for 3-RRR PPM

corresponds to a configuration where the three inner links are parallel and intersect at infinity. Special cases are illustrated in Figure 7.6.

\section{3-RRR}

Selecting the medial joints $\mathbf{p}_{4}, \mathbf{p}_{5}, \mathbf{p}_{6}$ to be actuated, their joint variables, as in (4.31), are $\phi_{i}=\theta_{i}-\theta_{i-3}, i=4,5,6$ and the input map $\hat{\pi}_{a}$ is the restriction to $\mathcal{C}$ of the projection

$$
\left(x_{i}, y_{i}, \theta_{i}\right)_{i=1, \ldots, 7} \mapsto\left[\theta_{4}-\theta_{1}, \theta_{5}-\theta_{2}, \theta_{6}-\theta_{3}\right]^{T}
$$

The resulting condition for input singularities reduces to

$\operatorname{det}\left[\begin{array}{cccccc}-l_{1} s_{1}-l_{4} s_{4} & 0 & 0 & 0 & 0 & -1 \\ l_{1} c_{1}+l_{4} c_{4} & 0 & 0 & 0 & -1 & 0 \\ 0 & -l_{2} s_{2}-l_{5} s_{5} & 0 & a_{1} s_{7} & 0 & -1 \\ 0 & l_{2} c_{2}+l_{5} c_{5} & 0 & -a_{1} c_{7} & -1 & 0 \\ 0 & 0 & -l_{3} s_{3}-l_{6} s_{6} & a_{2} s_{\alpha, 7} & 0 & -1 \\ 0 & 0 & l_{3} c_{3}+l_{6} c_{6} & -a_{2} c_{\alpha, 7} & -1 & 0\end{array}\right]=0$

This closely resembles the matrix given in (7.11) except the terms in the first three columns denote the coordinates of the leg vectors connecting corresponding base and platform anchor points $\ell_{i}:=\left(x_{i, i+3}, y_{i, i+3}\right)=\left(l_{i} c_{i}+\right.$ $\left.l_{i+3} c_{i+3}, l_{i} s_{i}+l_{i+3} s_{i+3}\right), i=1,2,3$. From Ceva's Theorem, the geometric 

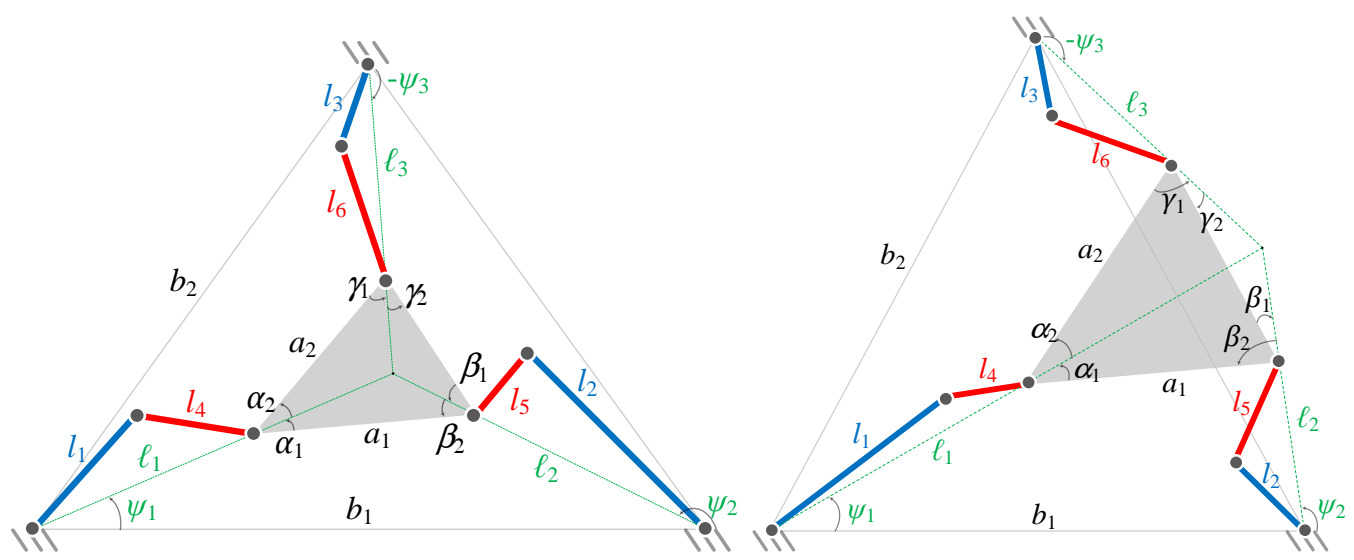

Figure 7.7: 3-RRR PPM input singularities

interpretation of this condition is that these vectors intersect (internally or externally) as in Figure 7.7.

\subsection{Planar 3-RPR parallel manipulator}

A fully general 3-RPR PPM is shown in Figure 7.8, along with a choice of moving and reference frames and design parameters. It is worth noting that the literature on this PPM generally assume that the direction of the prismatic joint is parallel to the line joining base and platform anchor points in each leg. However, we allow the P-joint to have arbitrary direction.

There are $k=7$ mobile links hence 21 pose variables, $t=9$ joints and nine design parameters, namely the P-joint offsets $d_{i}, i=1,2,3$, base parameters $b_{1}, b_{2}, \beta$, and the platform parameters $a_{1}, a_{2}, \alpha$. We make the non-triviality assumptions that $a_{i}, b_{i}>0, i=1,2$. The body coordinates of all joints in the relevant moving frames are

$$
\begin{array}{lll}
\mathbf{p}_{i}^{-}=(0,0)^{T}, & \mathbf{p}_{j}^{+}=(0,0)^{T}, & i=7,8,9 \quad j=1,2,3 \\
\mathbf{p}_{k}^{-}=\left(0, d_{k-3}\right)^{T}, & \mathbf{r}_{k}^{-}=\mathbf{r}_{k}^{+}=(1,0)^{T}, & k=4,5,6 \\
\mathbf{p}_{1}^{R}=(0,0)^{T}, & \mathbf{p}_{2}^{R}=\left(b_{1}, 0\right)^{T}, & \mathbf{p}_{3}^{R}=\left(b_{2} \cos \beta, b_{2} \sin \beta\right)^{T} \\
\mathbf{p}_{7}^{7}=(0,0)^{T}, & \mathbf{p}_{8}^{7}=\left(a_{1}, 0\right)^{T}, & \mathbf{p}_{9}^{7}=\left(a_{2} \cos \alpha, a_{2} \sin \alpha\right)^{T}
\end{array}
$$




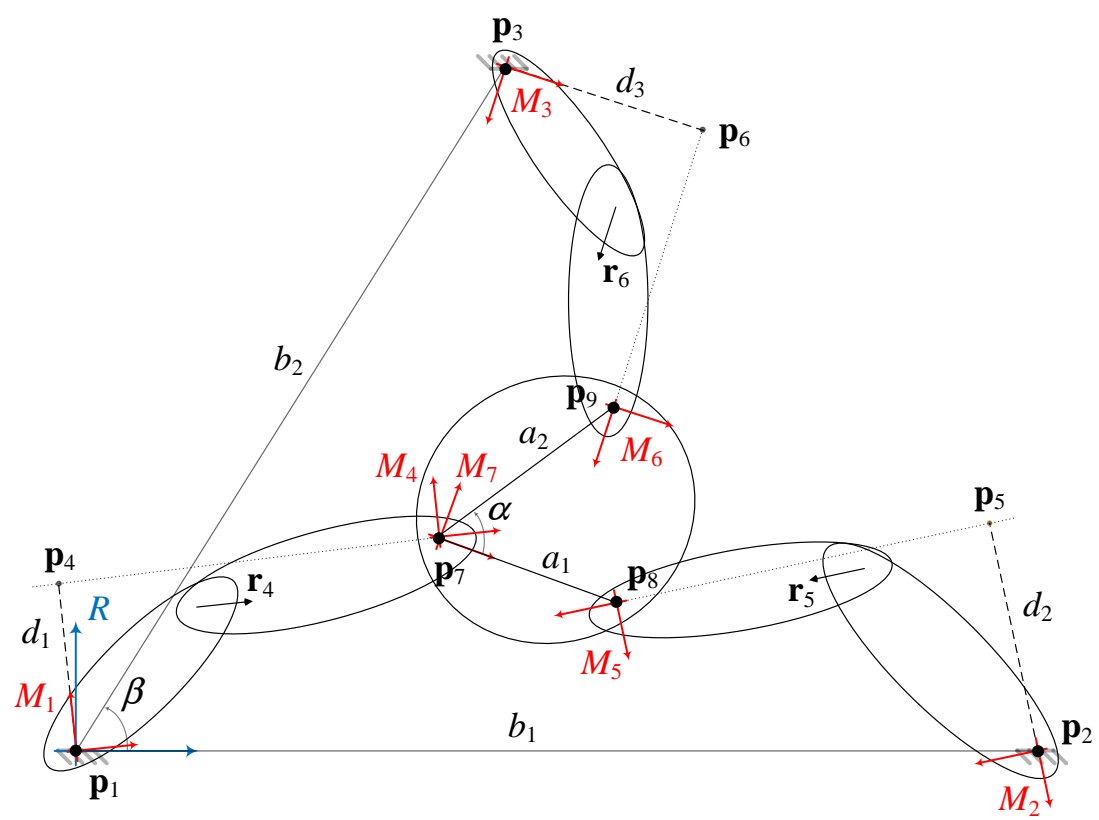

Figure 7.8: 3-RPR PPM with associated moving frames and design parameters

After transforming these body coordinates to the reference frame $R$, each P-joint imposes a pair of constraints of the form equation (4.15) while each R-joint imposes a pair of constraints of the form equation (4.12). Then, the $\mathrm{KCM} F: S E(2)^{7} \rightarrow \mathbb{R}^{18}$ is given by

$$
\begin{aligned}
\left(x_{i}, y_{i}, \theta_{i}\right)_{i=1, \ldots, 7} \mapsto[ & -x_{1},-y_{1}, b_{1}-x_{2},-y_{2}, b_{2} c_{\beta}-x_{3}, b_{2} s_{\beta}-y_{3}, x_{4,7}, y_{4,7}, \\
& x_{5,7}-a_{1} c_{7}, y_{5,7}-a_{1} s_{7}, x_{6,7}-a_{2} c_{\alpha, 7}, y_{6,7}-a_{2} s_{\alpha, 7}, \\
& s_{1,4}, s_{2,5}, s_{3,6}, d_{1}+y_{1,4} c_{1}-x_{1,4} s_{1}, d_{2}+y_{2,5} c_{2}-x_{2,5} s_{2}, \\
& \left.d_{3}+y_{3,6} c_{3}-x_{3,6} s_{3}\right]^{T}
\end{aligned}
$$

where $s_{i, i+3}=\sin \left(\theta_{i}-\theta_{i+3}\right), x_{i, i+3}=x_{i}-x_{i+3}, y_{i, i+3}=y_{i}-y_{i+3}, x_{i+3,7}=$ $x_{i+3}-x_{7}, y_{i+3,7}=y_{i+3}-y_{7}, i=1,2,3, c_{\alpha, 7}=\cos \left(\alpha+\theta_{7}\right)$ and $s_{\alpha, 7}=\sin \left(\alpha+\theta_{7}\right)$.

The associated Jacobian is an $(18 \times 21)$ matrix. Rather than solving first for rank deficiency, we can eliminate pose variables via the KCM; clearly from $s_{i, i+3}=0, \theta_{i}=\theta_{i+3} \bmod \pi$ for $i=1,2,3$. From this, for the purposes of rank computation the Jacobian can be reduced to a $(3 \times 6)$ matrix by row 
operations and deletions of rows and columns with leading 1s

$$
\left[\begin{array}{cccccc}
x_{1,4} c_{1}+y_{1,4} s_{1} & 0 & 0 & -s_{1} & c_{1} & 0 \\
0 & x_{2,5} c_{2}+y_{2,5} s_{2} & 0 & -s_{2} & c_{2} & a_{1} c_{2,7} \\
0 & 0 & x_{3,6} c_{3}+y_{3,6} s_{3} & -s_{3} & c_{3} & a_{2} c_{\alpha, 3,7}
\end{array}\right]
$$

where $c_{2,7}=\cos \left(\theta_{2}-\theta_{7}\right)$ and $c_{\alpha, 3,7}=\cos \left(\alpha-\theta_{3}+\theta_{7}\right)$. Columns in (7.18) correspond to partial derivatives with respect to $\theta_{1}, \theta_{2}, \theta_{3}, x_{7}, y_{7}, \theta_{7}$.

\subsubsection{Corank 1 singularities}

The conditions for a singularity are the vanishing of 19 non-trivial $(3 \times$ 3) minors of (7.18). Assuming the $\mathrm{KCM} F$ is well-behaved, dimensional considerations indicate that the corank 1 singular set $\Sigma_{1}^{1} F$ ought to have (at least) codimension 4 , so that four conditions on pose variables are expected. There are three cases that can readily be seen to result in singularity. Clearly, two of the non-trivial terms in the first three columns must vanish. Then, finding two further conditions is straightforward. For example, if the first two terms vanish then the condition for singularity is realised by

$$
x_{1,4} c_{1}+y_{1,4} s_{1}=x_{2,5} c_{2}+y_{2,5} s_{2}=0, \quad s_{1,2}=0, \quad c_{2,7}=0
$$

Further elimination of pose variables from (7.17) and (7.19) results in the following Grashof-type condition on design parameters

$$
\pm d_{1} \pm a_{1} \pm b_{1} \pm d_{2}=0
$$

Geometrically, this relates to the lower closed 2-RPR loop comprised of the base, two legs and platform. As shown in Figure 7.9a, It requires the two platform and two base anchor points to be collinear while the direction of the P-joints must be perpendicular to these. Similar configurations for the other two loops also correspond to singular configurations, see Figures $7.9 \mathrm{~b}$ 


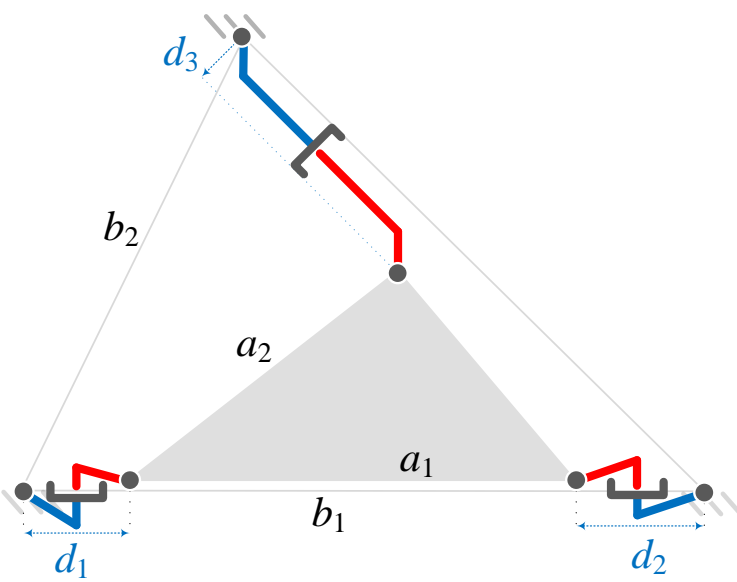

(a) Lower closed 2-RPR chain

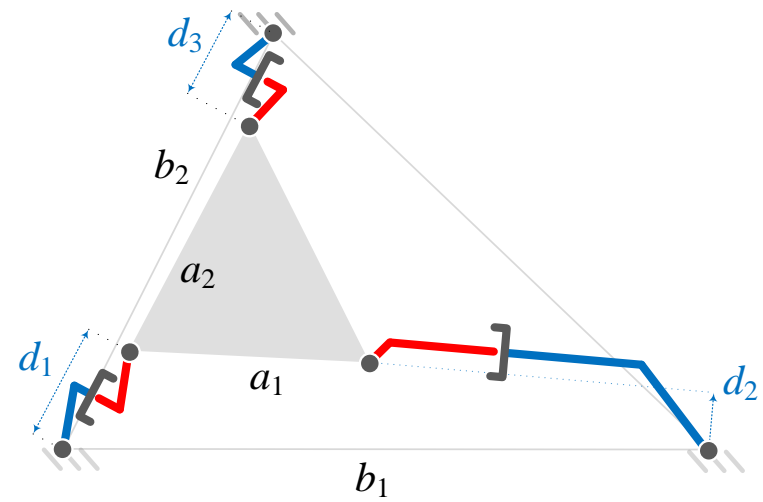

(b) Left closed 2-RPR chain

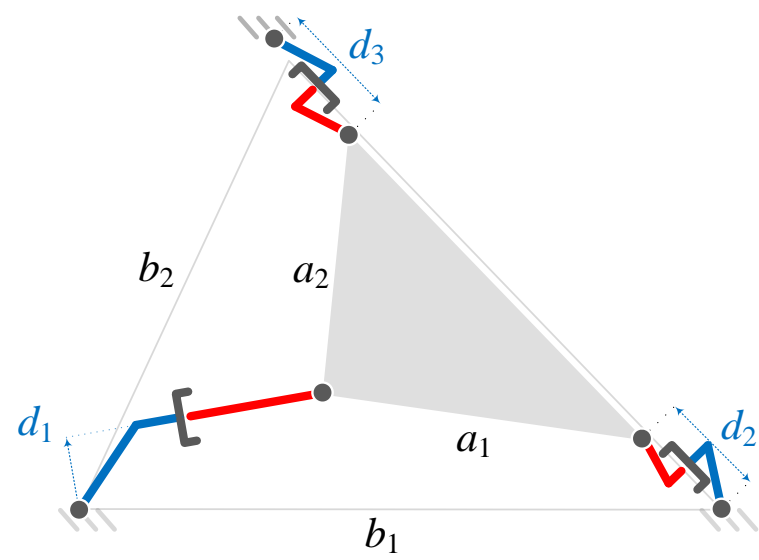

(c) Right closed 2-RPR chain

Figure 7.9: Corank 1 singular configurations for 3-RPR PPM 


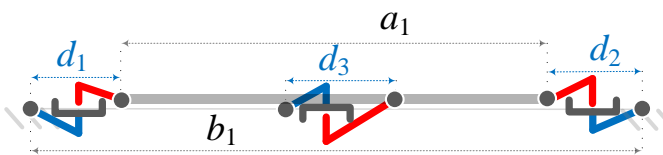

Figure 7.10: Corank 2 singular coonfigurations for 3-RPR PPM

and $7.9 \mathrm{c}$.

\subsubsection{Corank 2 singularities}

As explained in Section 7.1, there are other components of the singular set with higher codimension. Rather, we examine the conditions for the $\mathrm{KCM}$ to have a corank 2 singularity in the C-space. At such a point, the cokernel of the Jacobian has dimension 2. This can only happen for very special geometries of the $3-\mathrm{RPR}$ and is a high codimension condition. Necessary and sufficient conditions are that all $(2 \times 2)$ minors of $(7.18)$ vanish, but at least one entry is non-zero.There are 28 such 2 -minors leading to seven conditions as follows

$$
\begin{aligned}
& x_{1,4} c_{1}+y_{1,4} s_{1}=x_{2,5} c_{2}+y_{2,5} s_{2}=x_{3,6} c_{3}+y_{3,6} s_{3}=0, \\
& \theta_{1}=\theta_{2}=\theta_{3} \quad \bmod \pi, \quad c_{2,7}=c_{\alpha, 3,7}=0
\end{aligned}
$$

This is simply the union of the three closed-loop conditions giving corank 1 singularities. Thus, the three Grashof-type conditions like equation (7.20) must hold simultaneously and these entail $\alpha=\beta=0 \bmod \pi$, see Figure 7.10. These five equations correspond to four independent conditions.

\subsubsection{Output mapping and singularities}

Assuming displacement of the platform $L_{7}$ determines the workspace, the associated output mapping is of the form (7.7) as for the 3-RRR manipulator. To find output singularities, we compute the Jacobian of $\Pi_{w}:=\left(\hat{\pi}_{w}, F\right)$ : $\mathbb{R}^{21} \rightarrow \mathbb{R}^{18} \times \mathbb{R}^{3}$ which, after row operations and deletions of rows and columns 


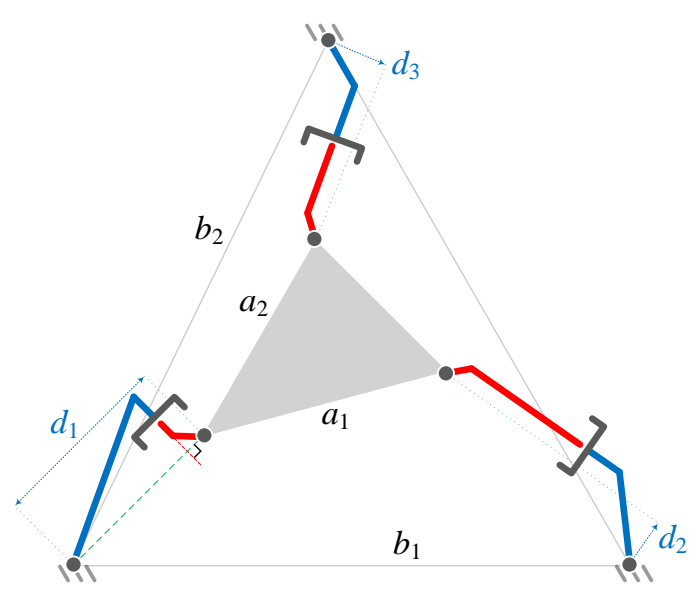

Figure 7.11: 3-RPR PPM output singularities

with leading $1 \mathrm{~s}$, simplifies to a $(6 \times 6)$ matrix

$$
\left[\begin{array}{cccccc}
0 & 0 & 0 & 0 & 0 & 1 \\
0 & 0 & 0 & 0 & 1 & 0 \\
0 & 0 & 0 & 1 & 0 & 0 \\
x_{1,4} c_{1}+y_{1,4} s_{1} & 0 & 0 & -s_{1} & c_{1} & 0 \\
0 & x_{2,5} c_{2}+y_{2,5} s_{2} & 0 & -s_{2} & c_{2} & a_{1} c_{2,7} \\
0 & 0 & x_{3,6} c_{3}+y_{3,6} s_{3} & -s_{3} & c_{3} & a_{2} c_{\alpha, 3,7}
\end{array}\right]
$$

Once again, the upper right $(3 \times 3)$ block is non-singular so we require the bottom left $(3 \times 3)$ block matrix to be rank-deficient requiring

$$
\left(x_{1,4} c_{1}+y_{1,4} s_{1}\right)\left(x_{2,5} c_{2}+y_{2,5} s_{2}\right)\left(x_{3,6} c_{3}+y_{3,6} s_{3}\right)=0
$$

Let vectors $\mathbf{v}_{i}=\left(x_{i, i+3}, y_{i, i+3}\right), i=1,2,3$ connect the R-joints in each leg, while $\mathbf{u}_{i}=\left(c_{i}, s_{i}\right)$ designate (unit) directions of the P-joints $\mathbf{r}_{i+3}, i=1,2,3$. Hence, an output singularity occurs if and only if $\xi_{i}:=\mathbf{v}_{i} \cdot \mathbf{u}_{i}=0$ for some $i=1,2,3$ i.e. one of the $\mathbf{v}_{i}$ is orthogonal to the corresponding joint direction or vanishes (so that base and anchor joints coincide), as shown in Figure 7.11 for the lower left leg. 

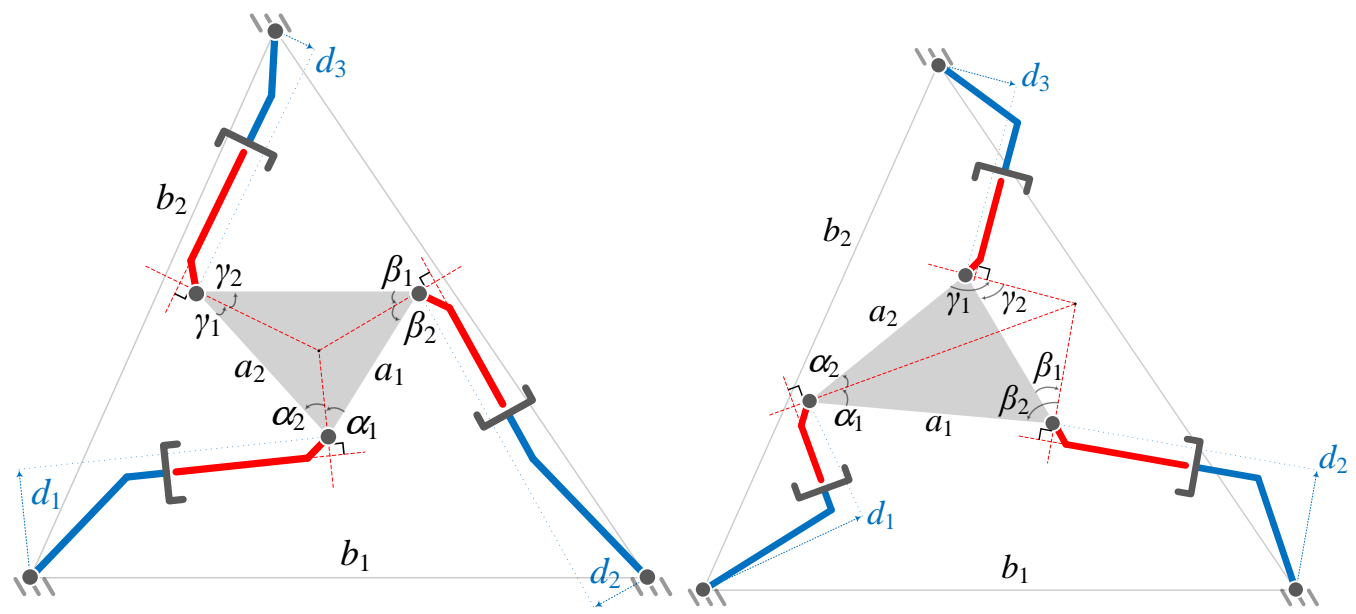

Figure 7.12: 3-RPR PPM input singularities

\subsubsection{Input mapping and singularities}

In this section, we will discuss two different possibilities for the actuated joints [28, 112]. First, we assume actuators are placed at the base R-joints i.e. $\mathbf{p}_{1}, \mathbf{p}_{2}, \mathbf{p}_{3}$. Secondly, we consider the medial P-joints i.e. $\mathbf{r}_{4}, \mathbf{r}_{5}, \mathbf{r}_{6}$ to be actuated

\section{3- $\underline{R P R}$}

Choosing the base R-joints $\mathbf{p}_{1}, \mathbf{p}_{2}, \mathbf{p}_{3}$ as actuators, as for the 3 - $\underline{R} R R$ in Section 7.1.3, the actuated joint variables are $\phi_{i}=\theta_{i}, i=1,2,3$. Applying Theorem 5.8, the $(21 \times 21)$ Jacobian matrix can then be reduced (by row operations and deletions of rows and columns with leading 1s) so that input singularities are determined by the $(3 \times 3)$ determinant

$$
\begin{aligned}
\operatorname{det}\left[\begin{array}{ccc}
-s_{1} & c_{1} & 0 \\
-s_{2} & c_{2} & a_{1} c_{2,7} \\
-s_{3} & c_{3} & a_{2} c_{\alpha, 3,7}
\end{array}\right]= & a_{1} \sin \left(\theta_{1}-\theta_{3}\right) \sin \left(\theta_{2}-\theta_{7}+\frac{\pi}{2}\right) \\
& +a_{2} \sin \left(\theta_{1}-\theta_{2}\right) \sin \left(\alpha-\theta_{3}+\theta_{7}+\frac{\pi}{2}\right)=0
\end{aligned}
$$

This corresponds to Ceva's condition (5.13), where $\psi_{a}=\theta_{1}+\frac{\pi}{2}, \psi_{b}=\theta_{2}+\frac{\pi}{2}$, $\psi_{c}=\theta_{3}+\frac{\pi}{2}$ and $\psi_{t}=\theta_{7}$. Geometrically, the lines through the platform 


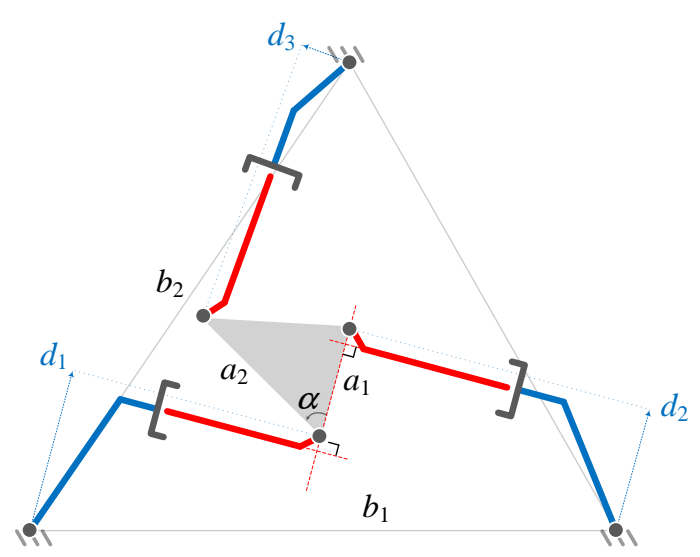

Figure 7.13: A special input singularity for 3-RPR PPM

anchor points and orthogonal to the P-joints $\mathbf{r}_{4}, \mathbf{r}_{5}, \mathbf{r}_{6}$ are concurrent, see Figure 7.12. This input singularity is independent of platform orientation $\theta_{7}$.

There is a special input-singular configuration if any of the following conditions is met

$$
\begin{array}{rr}
\theta_{1}=\theta_{2}=\theta_{7} & \bmod \pi / 2 \\
\theta_{1}=\theta_{3}=\alpha+\theta_{7} & \bmod \pi / 2 \\
\theta_{2}=\theta_{3}-\alpha=\theta_{7} & \bmod \pi / 2
\end{array}
$$

These special input singularities correspond to configurations where 2 Pjoints in a closed chain are parallel and perpendicular to a side of the platform. Figure 7.13 depicts, for example, a special input singularity of the bottom closed chain where $\mathbf{r}_{4}, \mathbf{r}_{5}$ are parallel and perpendicular to $a_{1}$.

\section{3-RPR}

Actuating the P-joints $\mathbf{r}_{4}, \mathbf{r}_{5}, \mathbf{r}_{6}$, so $\phi_{i}, i=4,5,6$ as given in equation (4.32), are joint variables, the input mapping can be taken as

$$
\left(x_{i}, y_{i}, \theta_{i}\right)_{i=1, \ldots, 7} \mapsto\left[\left(x_{1,4}^{2}+y_{1,4}^{2}\right),\left(x_{2,5}^{2}+y_{2,5}^{2}\right),\left(x_{3,6}^{2}+y_{3,6}^{2}\right)\right]^{T}
$$



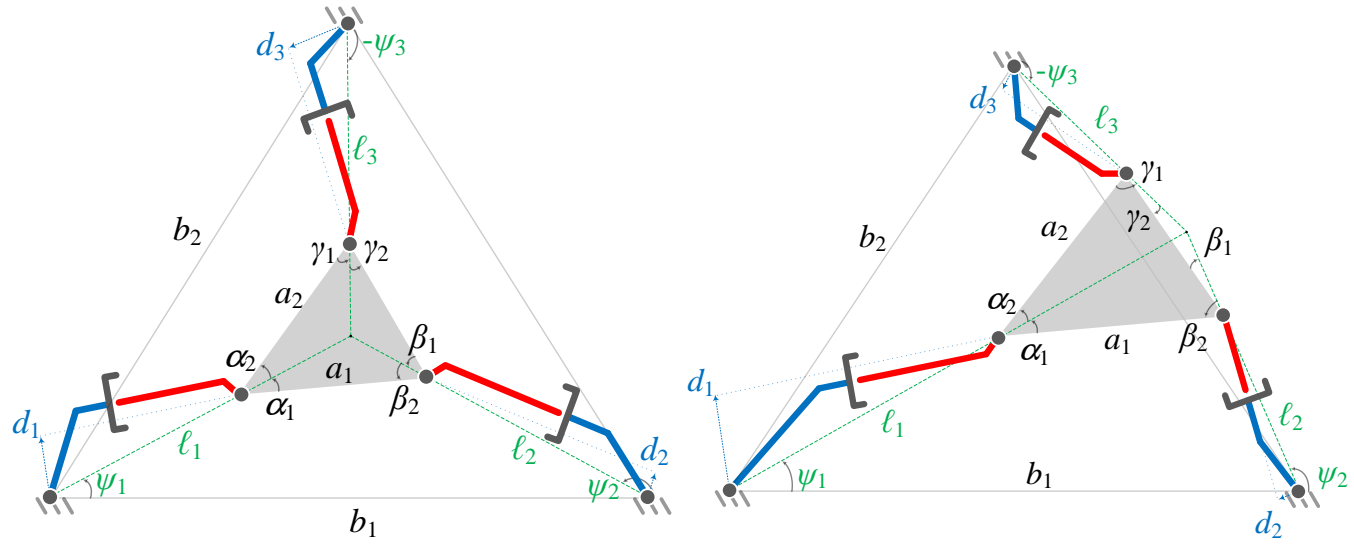

Figure 7.14: 3-Rㅛ input singularities

Theorem 5.8 yields a $(21 \times 21)$ Jacobian matrix which can be reduced and simplified to give the determinant

$\xi_{1} \xi_{2} \xi_{3}\left(a_{1} \sin \left(\psi_{1}-\psi_{3}\right) \sin \left(\theta_{7}-\psi_{2}\right)-a_{2} \sin \left(\psi_{1}-\psi_{2}\right) \sin \left(\alpha+\theta_{7}-\psi_{3}\right)\right)=0 \quad(7.27)$

where $\psi_{i}$ denote the angles of the legs $\ell_{i}$ (green dashed lines) with respect to the reference frame, as illustrated in Figure 7.14. Corresponding input singularities are given by (i) $\xi_{i}=0, i=1,2,3$, which are the output singularity conditions, or (ii) the vanishing of the bracket, which by Ceva's condition (5.13), corresponds to the extensions of the leg vectors being concurrent. 


\section{Chapter 8}

\section{Planar geared manipulators}

The use of gear pairs in a mechanism may confer a number of advantages. For example, they can enable more efficient placement of the actuators, thereby reducing their mass and inertia. Epicyclic gear trains (EGTs), in which the centre of one gear wheel revolves around that of another, are the simplest form and therefore play an important role in geared mechanisms (GMs). By utilising EGTs, we can easily place actuators close to the base of a GM and rotation of inputs can be efficiently transmitted to the end-effector. Careful choice of gear ratios can also enable end-effector motion to be tailored to specific inputs.

The fundamental kinematic equations for an epicyclic gear are due to Willis [113]. Subsequent authors have introduced methods of global analysis for GMs that ensure that the equations are correctly formulated for a given mechanism topology and design. Notably, Buchsbaum and Freudenstein [114] introduced combinatoric methods to represent the topology of the mechanism. This approach was later developed by Tsai [86], Hsu and Lam [115]. In order to enhance the computational effectiveness of the method, Talpasanu et al. [87] refined and to some extent recast the approach, introducing the 'incidence and transfer method' that uses the cycle matroid of the mechanism's directed graph. A comparison of Talpasanu's method with that 


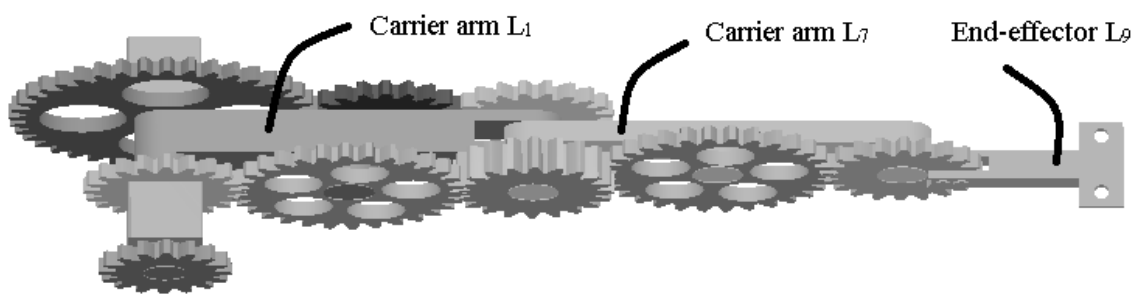

Figure 8.1: 3-dof geared planar manipulator

of Tsai-Tokad was made in [88]. In this Chapter, we illustrate Talpasanu's method for a simple geared version of a planar 3R mechanism in order to determine its kinematic mapping and thereby its singularities.

There does not appear to be an established literature on singularities of geared mechanisms. For this reason, we explore both a traditional inputoutput kinematic model and a constraint-based approach. While the example presented is straightforward, it is intended to provide a model for extending singularity analysis of GMs to more complex cases, including those which are genuinely spatial in their kinematics and to parallel mechanisms incorporating gearing.

\subsection{The mechanism}

A simple planar GM consists of $n+1$ links, $L_{0}, \ldots, L_{n}$, and $m$ joints that include $t$ revolute (turning) pairs, $T_{1}, \ldots, T_{t}$, and $g$ gear pairs, $G_{1}, \ldots, G_{g}$, so that $m=t+g$. Note that the number of links, excluding the base $L_{0}$, is assumed equal to the number of simple pairs, i.e. $t=n$. In effect, the mechanism without gears contains no closed chains.

By placing three actuator joints at the base and using simple spur-gear pairs to transmit motion to the end-effector, one obtains a geared mechanism based on a simple serial planar 3R mechanism (see Figure 8.1). One EGT, consisting of three gear wheels and using link $L_{1}$ as carrier, transmits motion to the link $L_{7}$, while a second EGT of five gear wheels with links $L_{1}$ and $L_{7}$ 


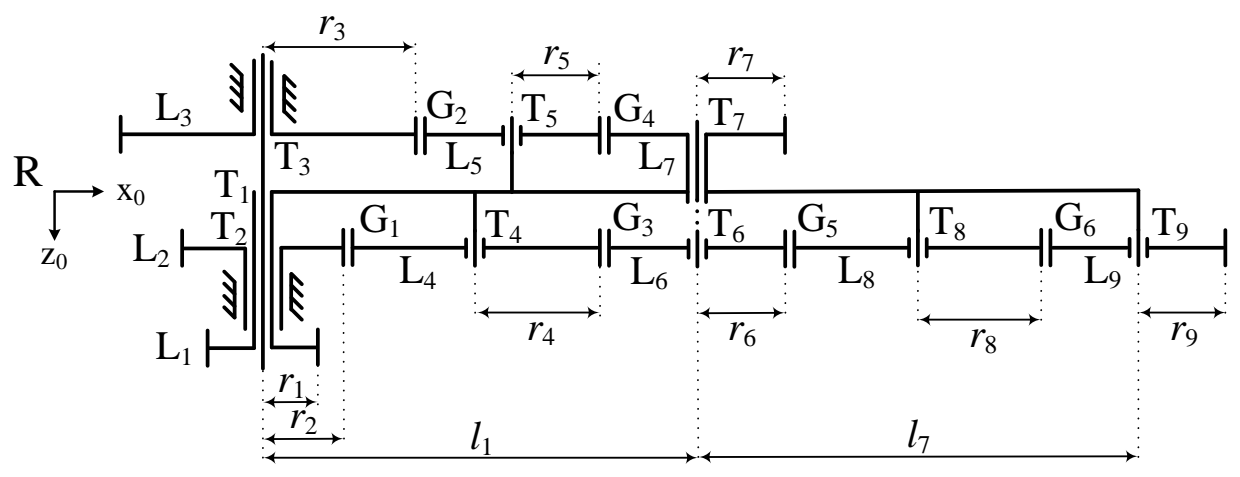

Figure 8.2: Functional schematic of the manipulator in Figure 8.1

as carriers transmits motion to the end-effector.

A functional schematic for the mechanism is illustrated in Figure 8.2. The inputs, which are attached to the base $L_{0}$, are via $T_{i}, i=1,2,3$ while link $L_{9}$ is the output planet gear or end-effector. Note that the carrier arms $L_{1}$ and $L_{7}$ that form the first two links in the underlying planar $3 \mathrm{R}$ are also gear wheels. Other links are intermediate (idler) gear wheels.

In its directed graph (digraph) representation, Figure 8.3, the links (including gear wheels) are vertices $\left(L_{0}, \ldots, L_{9}\right)$ while joints are edges. Specifically, the revolute pairs $\left(T_{1}, \ldots, T_{9}\right)$ are solid edges and gear pairs $\left(G_{1}, \ldots\right.$, $\left.G_{6}\right)$ are dashed. Note that the solid edges form a spanning tree for the graph; put another way, each simple cycle contains at least one gear pair as an edge. The direction of an edge connecting vertices (links) $L_{i}$ and $L_{j}$ is $L_{i} \rightarrow L_{j}$ if the transmission from input to output flows from $L_{i}$ to $L_{j}$.

Application of the CGK formula, given in (4.1), shows that the GM has three degrees of freedom, noting that a gear pair has two degrees of freedom. Talpasanu [87] and Tsai [86] also observe that there is a relation between the degrees of freedom of the GM, the number of links and the number of gear pairs

$$
\delta=n-g
$$

again yielding $\delta=3$. 


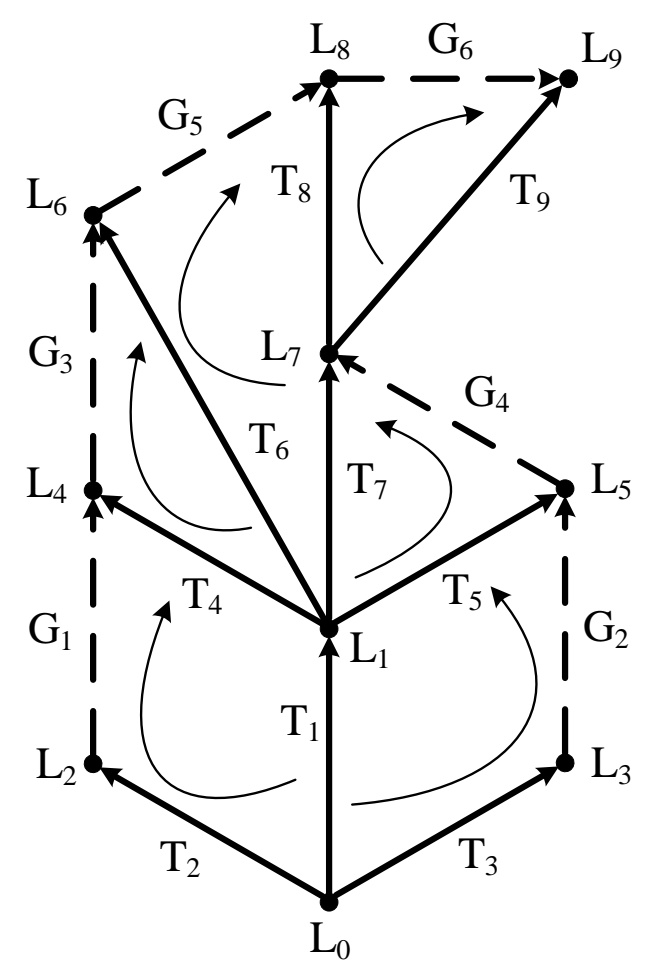

Figure 8.3: Associated digraph

\subsection{Input-output analysis via the matroid method}

To perform the standard input-output kinematic analysis, we apply the matroid method of Talpasanu [87] to obtain the Willis kinematic equations for all gear pairs and solve these equations to express all joint variables in terms of the input variables. This enables us to express the forward kinematic mapping as a product of exponentials (PoEs) in terms of input variables alone and consequently to undertake the singularity analysis. It is worth noting that the Willis equations usually express the relation between angular velocities in a gear-pair/carrier cycle but since the relations between the joint variables themselves are linear, the same equations hold between the underlying variables as between their velocities.

There are essentially three stages to the matroid or incidence-transfer method: the first stage codifies the topology of the digraph representation 
of the GM in matrix form. The second stage builds the specific design on to this by introducing dimensions that can then be interpreted as gear ratios. The method ensures that we obtain a minimal set of linear (Willis) equations and the third stage is to solve these for the joint variables in terms of the input variables.

Associated to the digraph are two matrices. The incidence matrix $\Pi^{0}$ has rows labelled by the vertices and columns by edges and its entries $\pi_{i j}^{0}$ are -1 or 1 according as edge $j$ leaves or enters vertex $i$, or else is 0 . In this setting, the base $L_{0}$ is fixed and its row (containing only -1 and 0 ) is linearly dependent on the other rows. So, for the purpose of analysis we omit this row and arrive at the reduced incidence matrix $\Pi_{n \times m}$, which can be partitioned into submatrices

$$
\begin{aligned}
& \Pi=\left[P_{n \times t} \mid \hat{P}_{n \times g}\right] \\
& \begin{array}{llllllllllllllll}
T_{1} & T_{2} & T_{3} & T_{4} & T_{5} & T_{6} & T_{7} & T_{8} & T_{9} & G_{1} & G_{2} & G_{3} & G_{4} & G_{5} & G_{6}
\end{array}
\end{aligned}
$$

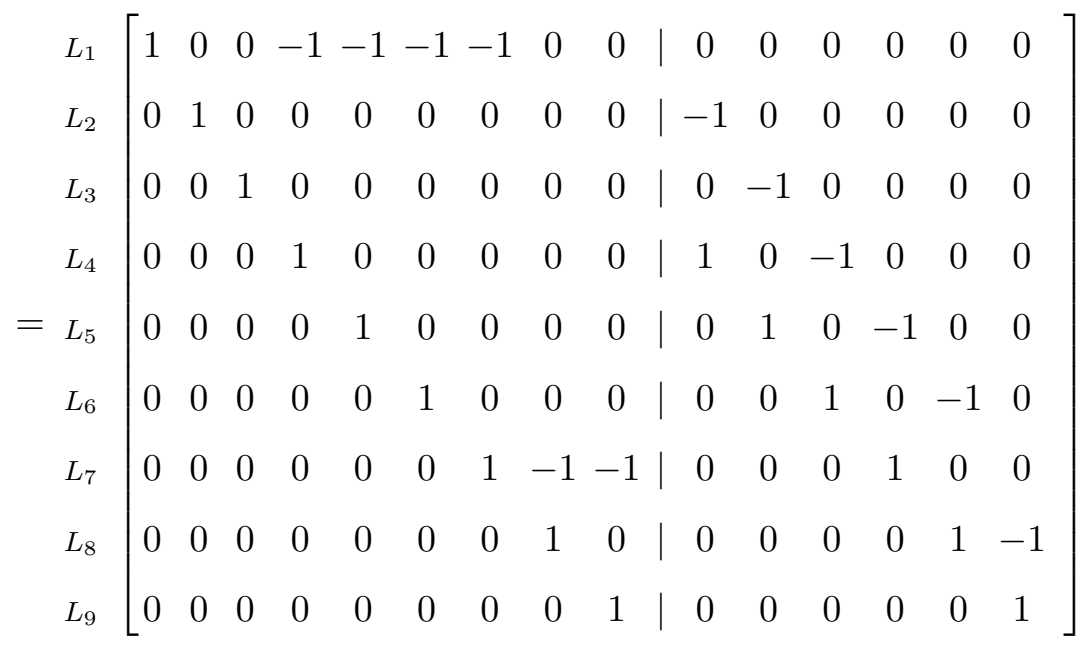

A cycle basis matrix $\Gamma$ for a digraph consists of a maximally independent set of rows $G_{i}, i=1, \ldots, g$, each row corresponding to a cycle, whose entries $\gamma_{i j}, j=1, \ldots, m$ are -1 or 1 according to whether edge $j$ appears in that cycle directed with, or opposed to, a given vertex order for the cycle, or otherwise 0 . The cycle space is in fact the nullspace of the incidence matrix so, according to Euler's formula, its dimension is $m-n$. Given the special 
structure of the digraph for a GM, we have $m-n=g$ and a basis for the cycle space can be indexed by the gear pairs, $G_{1}, \ldots, G_{g}$. For the given GM, with the vertex order as indicated in Figure 8.3 by arrows in each basis cycle, $\Gamma$ can be partitioned into submatrices

$$
\begin{aligned}
& \Gamma_{g \times m}=\left[C_{g \times t} \mid I_{g \times g}\right] \\
& \begin{array}{lllllllllllllllll}
T_{1} & T_{2} & T_{3} & T_{4} & T_{5} & T_{6} & T_{7} & T_{8} & T_{9} & G_{1} & G_{2} & G_{3} & G_{4} & G_{5} & G_{6}
\end{array}
\end{aligned}
$$

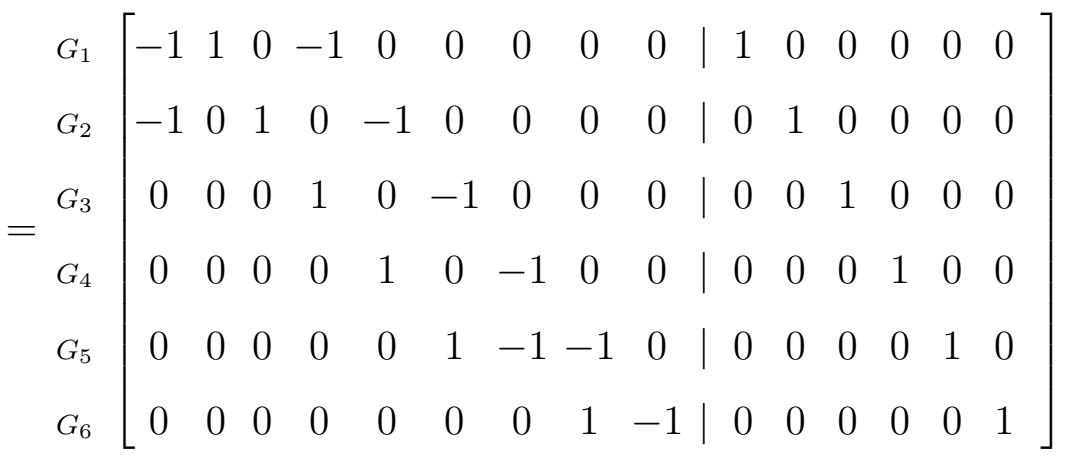

where the second block is the identity matrix. Note that $\Gamma$ is the cycle basis matrix corresponding to the specific spanning tree for the digraph, which one can obtain by deleting the dashed lines in Figure 8.3. In any graph with edge set $E$, the collection $I$ of subsets of $E$ that do not include a cycle form a matroid - mathematical objects that capture the abstract idea of independence. Spanning trees are maximally independent while simple cycles are minimally dependent objects $[116,87]$.

The second step is to introduce design parameters into the matrices. The constraint imposed by the cycles on the motion of the GM is captured by the joint position matrix $\Delta$ whose entries are $\delta_{i j}=c_{i j} d_{i j}$, where $c_{i j}$ are components of the (reduced) cycle basis matrix $C$ and $d_{i j}=y_{T_{j}}-y_{G_{i}}$ where $y_{T_{j}}, j=1, \ldots, t$ and $y_{G_{i}}, i=1, \ldots, g$ are distances of the axes of turning joint $T_{j}$ and meshing joint $G_{i}$ from the base in home configuration. These 
distances are the radii of the various gear wheels $r_{k}, k=2, \ldots, t$ so that

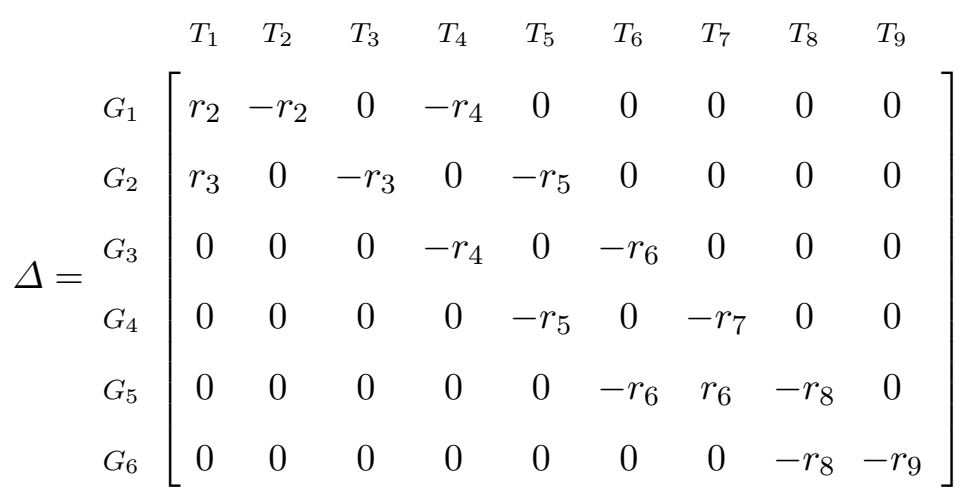

For an oriented gear pair $G_{i}$, some $i=1, \ldots, g$, connecting link (gear wheel) $L_{p}$ to $L_{q}$, denote the corresponding gear ratio

$$
\rho_{i}:=\frac{-r_{p}}{r_{q}}
$$

The rows of the matrix represent equations that hold between the joint variables at each revolute pair (or equivalently their angular velocities) so that each row can be independently scaled by one of the radii to realise the gear ratio matrix

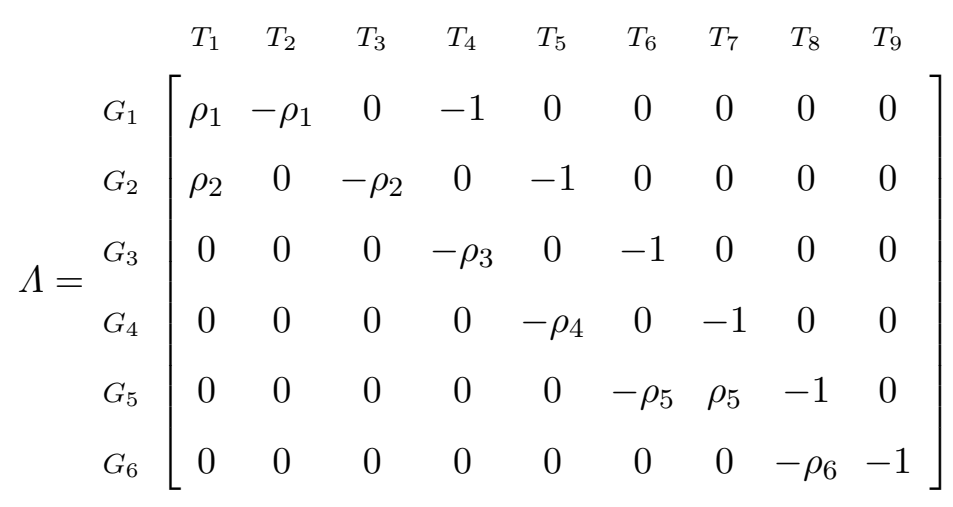

To arrive finally at a complete set of Willis equations for the GM, it is necessary to incorporate the component $P$ of the reduced incidence matrix that provides the connection between the angles of rotation $\theta_{i}$ for each link $L_{i}$ and the joint variables $\phi_{j}$ at each revolute pair $T_{j}, i, j=1, \ldots, n(=t$ as noted in Section 8.1). Specifically, set $\Sigma_{g \times t}=\Lambda_{g \times t} P_{t \times t}^{T}$, then the Willis 
equations have the matrix form

$$
\Sigma \boldsymbol{\theta}=\mathbf{0}
$$

where $\boldsymbol{\theta}$ is the vector of link rotations and can be partitioned between input variables and passive variables. Following (8.1), there are three input variables and six passive variables. Explicitly

$$
\boldsymbol{\theta}=\left[\boldsymbol{\theta}_{\delta} \mid \boldsymbol{\theta}_{g}\right]^{T}=\left[\begin{array}{llllllllll}
\theta_{1} & \theta_{2} & \theta_{3} & \theta_{4} & \theta_{5} & \theta_{6} & \theta_{7} & \theta_{8} & \theta_{9}
\end{array}\right]^{T}
$$

Partitioning $\Sigma$ in a similar way, and expanding the product gives

$$
\begin{aligned}
& \Sigma=\left[Z_{\delta} \mid Z_{g}\right] \\
& \begin{array}{llllllllll}
L_{1} & L_{2} & L_{3} & L_{4} & L_{5} & L_{6} & L_{7} & L_{8} & L_{9}
\end{array}
\end{aligned}
$$

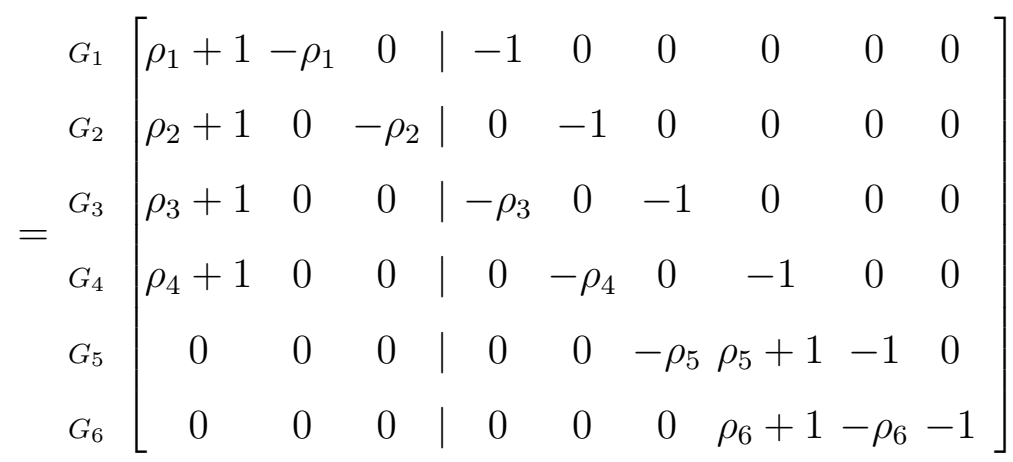

Now, we can rewrite equation (8.7) in the form of

$$
\left[Z_{\delta} \mid Z_{g}\right]\left[\begin{array}{l}
\boldsymbol{\theta}_{\delta} \\
\boldsymbol{\theta}_{g}
\end{array}\right]=\mathbf{0}
$$

from which it follows that, provided $Z_{g}$ is non-singular, which is easily verified in this case,

$$
\boldsymbol{\theta}_{g}=-Z_{g}^{-1} Z_{\delta} \boldsymbol{\theta}_{\delta}
$$

Solving equation (8.10), gives passive variables in terms of input variables as 
follows

$$
\left[\begin{array}{c}
\theta_{4} \\
\theta_{5} \\
\theta_{6} \\
\theta_{7} \\
\theta_{8} \\
\theta_{9}
\end{array}\right]=\left[\begin{array}{ccc}
\rho_{1}+1 & -\rho_{1} & 0 \\
\rho_{2}+1 & 0 & -\rho_{2} \\
1-\rho_{1} \rho_{3} & \rho_{1} \rho_{3} & 0 \\
1-\rho_{2} \rho_{4} & 0 & \rho_{2} \rho_{4} \\
\rho_{1} \rho_{3} \rho_{5}-\rho_{2} \rho_{4}\left(1+\rho_{5}\right)+1 & -\rho_{1} \rho_{3} \rho_{5} & \rho_{2} \rho_{4}\left(1+\rho_{5}\right) \\
-\rho_{1} \rho_{3} \rho_{5} \rho_{6}+\rho_{2} \rho_{4}\left(\rho_{5} \rho_{6}-1\right)+1 & \rho_{1} \rho_{3} \rho_{5} \rho_{6} & -\rho_{2} \rho_{4}\left(\rho_{5} \rho_{6}-1\right)
\end{array}\right]\left[\begin{array}{c}
\theta_{1} \\
\theta_{2} \\
\theta_{3}
\end{array}\right]
$$

\subsection{KCM and C-space singularities}

In the previous section, we showed how to find relations between turning pairs in a GM by using the matroid method. In this section, we are going to determine the KCM corresponding to the GM in Figure 8.2 and then examine whether there are C-space, input, and output singularities or not.

There are two ways we could approach the GM using the KCM approach. It is possible to do the analysis from scratch. As in Figure 8.1, the manipulator has nine mobile links, $L_{1}, \ldots, L_{9}$, including gear wheels. It has nine R-joints, $T_{1}, \ldots, T_{9}$ and six gear pairs, $G_{1}, \ldots, G_{6}$. The domain of the KCM should be $S E(2)^{9}$ with 27 pose variables. The R-joints give 18 constraint equations, with nine pairs as in (4.13). So far, we have not discussed constraint equations for gear pairs. Since each pair has two degrees of freedom, we have only one constraint. This is given in terms of the gear ratio (8.5). Since the relative rotations of the gear pair are related by this, if the gear pair $G_{i}$ involves links $L_{p}$ and $L_{q}$ with gear ratio $\rho_{i}$, then

$$
\theta_{p}=\rho_{i} \theta_{q}
$$

This gives a KCM $F: S E(2)^{9} \rightarrow \mathbb{R}^{24}$. Indeed, it is relatively straightforward to compute its Jacobian and observe that it always has maximum rank.

On the other hand, we have already shown how to eliminate the passive joint variables using (8.11) so a simpler approach is to find the KCM for 


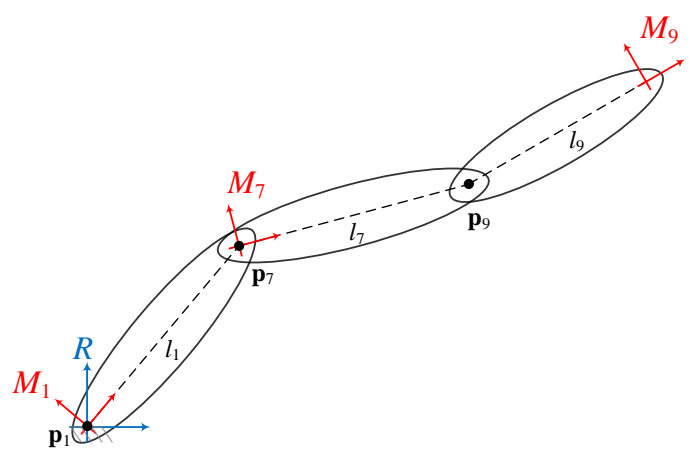

Figure 8.4: Planar GM with associated frames and links' lengths

the underlying serial manipulator determined by the carrier arms $L_{1}, L_{7}$ and end-effector $L_{9}$. Ultimately, the two approaches can be seen to be equivalent by eliminating variables and constraints.

As shown in Figure 8.4, the planar GM is based on the serial manipulator consisting of four rigid bodies $L_{0}, L_{1}, L_{7}, L_{9}$ which are connected via planar R-joints $T_{1}, T_{7}, T_{9}$. Assume $L_{0}$ is fixed, so there are totally nine pose variables and three design parameters (link lengths) $l_{i}, i=1,7,9$.

Based on the choice of reference and moving frames, body coordinates of three revolute joints in $M_{ \pm}$frames are

$$
\begin{aligned}
& \mathbf{p}_{1}^{R}=(0,0)^{T}, \quad \mathbf{p}_{7}^{1}=\left(l_{1}, 0\right)^{T}, \quad \mathbf{p}_{9}^{7}=\left(l_{7}, 0\right)^{T} \\
& \mathbf{p}_{1}^{1}=(0,0)^{T}, \quad \mathbf{p}_{7}^{7}=(0,0)^{T}, \quad \mathbf{p}_{9}^{9}=\left(-l_{9}, 0\right)^{T}
\end{aligned}
$$

Reference-frame coordinates of each joint can be obtained using (4.11). Each joint imposes a pair of constraint equations like those given in (4.12). Therefore, the $\mathrm{KCM} F: S E(2)^{3} \rightarrow \mathbb{R}^{6}$ can be defined in terms of pose variables

$$
\begin{gathered}
\left(x_{i}, y_{i}, \theta_{i}\right)_{i=1,7,9} \mapsto\left[-x_{1},-y_{1}, x_{1}-x_{7}+l_{1} c_{1}, y_{1}-y_{7}+l_{1} s_{1}, x_{7}-x_{9}+l_{7} c_{7}+l_{9} c_{9},\right. \\
\left.y_{7}-y_{9}+l_{7} s_{7}+l_{9} s_{9}\right]^{T}
\end{gathered}
$$

Taking derivatives of $(8.13)$ results in a $(6 \times 9)$ Jacobian matrix which can be reduced to a $(2 \times 5)$ matrix by row operations and deletions of rows 
and columns with leading 1s

$$
J_{1} F^{\mathrm{red}}=\left[\begin{array}{ccccc}
-l_{1} s_{1} & -l_{7} s_{7} & -1 & 0 & -l_{9} s_{9} \\
l_{1} c_{1} & l_{7} c_{7} & 0 & -1 & l_{9} c_{9}
\end{array}\right]
$$

Clearly, this matrix is non-singular because it contains one $(2 \times 2)$ minor whose determinant equals one. As a result, C-space singularities never occur. This is an example to show that the C-space of a serial manipulator is always a smooth manifold.

Since $\operatorname{dim} \mathcal{C}=3$, three actuated joints are required, in the serial manipulator, these are revolute pairs $T_{1}, T_{7}, T_{9}$. As in (4.31), their joint variables are $\phi_{1}=\theta_{1}, \phi_{7}=\theta_{7}-\theta_{1}$ and $\phi_{9}=\theta_{9}-\theta_{7}$, respectively. Thus, its input map $\hat{\pi}_{a}$ is the restriction to $\mathcal{C}$

$$
\left(x_{i}, y_{i}, \theta_{i}\right)_{i=1,7,9} \mapsto\left(\theta_{1}, \theta_{7}-\theta_{1}, \theta_{9}-\theta_{7}\right)
$$

However, the GM inputs are $\theta_{1}, \theta_{2}, \theta_{3}$ and using (8.11), we can replace $\theta_{7}, \theta_{9}$ in $\Pi_{a}:=\left(\hat{\pi}_{a}, F\right)$ by these inputs

$$
\begin{aligned}
\left(x_{i}, y_{i}, \theta_{1}, \theta_{2}, \theta_{3}\right)_{i=1,7,9} \mapsto & -x_{1},-y_{1}, x_{1}-x_{7}+l_{1} c_{\theta_{1}}, y_{1}-y_{7}+l_{1} s_{\theta_{1}}, x_{7}-x_{9} \\
& +l_{7} c_{\alpha_{1} \theta_{1}} c_{\alpha_{3} \theta_{3}}+l_{9} c_{\beta_{1} \theta_{1}} c_{\beta_{2} \theta_{2}} c_{\beta_{3} \theta_{3}}-l_{9} c_{\beta_{3} \theta_{3}} s_{\beta_{1} \theta_{1}} s_{\beta_{2} \theta_{2}} \\
& -l_{7} s_{\alpha_{1} \theta_{1}} s_{\alpha_{3} \theta_{3}}-l_{9} c_{\beta_{2} \theta_{2}} s_{\beta_{1} \theta_{1}} s_{\beta_{3} \theta_{3}}-l_{9} c_{\beta_{1} \theta_{1}} s_{\beta_{2} \theta_{2}} s_{\beta_{3} \theta_{3}}, \\
& y_{7}-y_{9}+l_{7} c_{\alpha_{3} \theta_{3}} s_{\alpha_{1} \theta_{1}}+l_{9} c_{\beta_{2} \theta_{2}} c_{\beta_{3} \theta_{3}} s_{\beta_{1} \theta_{1}} \\
& +l_{9} c_{\beta_{1} \theta_{1}} c_{\beta_{3} \theta_{3}} s_{\beta_{2} \theta_{2}}+l_{7} c_{\alpha_{1} \theta_{1}} s_{\alpha_{3} \theta_{3}}+l_{9} c_{\beta_{1} \theta_{1}} c_{\beta_{2} \theta_{2}} s_{\beta_{3} \theta_{3}} \\
& -l_{9} s_{\beta_{1} \theta_{1}} s_{\beta_{2} \theta_{2}} s_{\beta_{3} \theta_{3}}, \theta_{1},-\theta_{1}+\alpha_{1} \theta_{1}+\alpha_{3} \theta_{3},-\alpha_{1} \theta_{1} \\
& \left.+\beta_{1} \theta_{1}+\beta_{2} \theta_{2}-\alpha_{3} \theta_{3}+\beta_{3} \theta_{3}\right]^{T}
\end{aligned}
$$

where $\alpha_{1}=1-\rho_{2} \rho_{4}, \alpha_{3}=\rho_{2} \rho_{4}, \beta_{1}=-\rho_{1} \rho_{3} \rho_{5} \rho_{6}+\rho_{2} \rho_{4}\left(\rho_{5} \rho_{6}-1\right)+1$, $\beta_{2}=\rho_{1} \rho_{3} \rho_{5} \rho_{6}$, and $\beta_{3}=-\rho_{2} \rho_{4}\left(\rho_{5} \rho_{6}-1\right)$. Taking derivatives of $\Pi_{a}$ results in a $(9 \times 9)$ Jacobian matrix which can be reduced (by row operations and deletions of rows and columns with leading 1s) so that input singularities are 
determined by the $(5 \times 5)$ determinant

$$
\begin{aligned}
& \operatorname{det}\left[\begin{array}{ccccc}
-1 & 0 & -l_{1} s_{1}-l_{7} \alpha_{1} s_{1,3}-l_{9} \beta_{1} s_{1,2,3} & -l_{9} \beta_{2} s_{1,2,3} & -l_{7} \alpha_{3} s_{1,3}-l_{9} \beta_{3} s_{1,2,3} \\
0 & -1 & l_{1} c_{1}+l_{7} \alpha_{1} c_{1,3}+l_{9} \beta_{1} c_{1,2,3} & l_{9} \beta_{2} c_{1,2,3} & l_{7} \alpha_{3} c_{1,3}+l_{9} \beta_{3} c_{1,2,3} \\
0 & 0 & 1 & 0 & 0 \\
0 & 0 & \alpha_{1}-1 & 0 & \alpha_{3} \\
0 & 0 & \beta_{1}-\alpha_{1} & \beta_{2} & \beta_{3}-\alpha_{3}
\end{array}\right] \\
& =-\alpha_{3} \beta_{2}
\end{aligned}
$$

where $c_{1,3}=\cos \left(\alpha_{1} \theta_{1}+\alpha_{3} \theta_{3}\right)$ and $c_{1,2,3}=\cos \left(\beta_{1} \theta_{1}+\beta_{2} \theta_{2}+\beta_{3} \theta_{3}\right)$ and similarly for sines. Assume $\alpha_{3}, \beta_{2}>0$ (they are products of gear ratios), this non-zero determinant shows that for a serial manipulator like the one in Figure 8.4 there are no input singularities.

Since the displacement of the end-effector $L_{9}$ is planar and represented by an element of $S E(2)$, so $\operatorname{dim} \mathcal{W}=3$. Hence, the $\mathrm{W}$-space is parametrised by $\left(x_{9}, y_{9}, \theta_{9}\right)$ and the output map is the restriction of $\hat{\pi}_{w}$ to $\mathcal{C}$. For identifying output singularities, we follow Theorem 5.8 so

$$
\Pi_{w}:=\left(\hat{\pi}_{w}, F\right):\left(x_{i}, y_{i}, \theta_{i}\right)_{i=1,7,9} \rightarrow\left(x_{9}, y_{9}, \theta_{9}, F\left(x_{1}, \ldots, \theta_{9}\right)\right)
$$

The Jacobian of $\Pi_{w}$ can be reduced (by row operations and deletions of rows and columns with leading 1 s) to a $(5 \times 5)$ matrix whose determinant is used to detect output singularities

$$
\operatorname{det}\left[\begin{array}{ccccc}
0 & 0 & 1 & 0 & 0 \\
0 & 0 & 0 & 1 & 0 \\
0 & 0 & 0 & 0 & 1 \\
-l_{1} s_{1} & -l_{7} s_{7} & -1 & 0 & -l_{9} s_{9} \\
l_{1} c_{1} & l_{7} c_{7} & 0 & -1 & l_{9} c_{9}
\end{array}\right]=-l_{1} l_{7} \sin \left(\theta_{1}-\theta_{7}\right)
$$

Clearly, an output singularity occurs if $\theta_{1}=\theta_{7} \bmod \pi$-geometrically, when 
$L_{1}, L_{7}$ are aligned.

In the next section, we are going to study FKP based on the input-output relation which is formulated by using a PoEs. We will show that singularities corresponding to FKP include both input and output singularities. However since the input mapping is non-singular, so the FKP singularities only depend on the output map.

\subsection{Forward kinematic and singularity anal- ysis}

The forward kinematic map of the mechanism can be written in terms of the revolute pair rotations as a product of exponentials (PoE) in the relevant Euclidean group (see Murray et al. [80]). In this case, as the mechanism is planar, the group is $S E(2)$. The form of PoE derives from the corresponding open-loop chain (see Tsai [117]) as follows

$$
T(\boldsymbol{\phi})=e^{X_{1} \phi_{1}} e^{X_{7} \phi_{7}} e^{X_{9} \phi_{9}} T(\mathbf{0})
$$

where $T(\mathbf{0})$ is the transformation between base and end-effector frames at the rest position $\boldsymbol{\phi}=\mathbf{0}$ and $X_{i}$ denote the infinitesimal rotations of revolute joints $T_{i}, i=1,7,9$ about their centres of rotation. Explicitly, we can use homogeneous representations as follows

$$
X_{i}=\left[\begin{array}{ccc}
0 & -1 & 0 \\
1 & 0 & -\xi_{i} \\
0 & 0 & 0
\end{array}\right]
$$

where (with respect to appropriate choices of body coordinates) $\xi_{1}=0$, $\xi_{7}=l_{1}, \xi_{9}=l_{1}+l_{7}$ with $l_{1}=r_{2}+2 r_{4}+r_{6}=r_{3}+2 r_{5}+r_{7}$ and $l_{7}=r_{6}+2 r_{8}+r_{9}$ 
the lengths of the carrier arms $L_{1}$ and $L_{7}$ (see Figure 8.2); and

$$
T(\mathbf{0})=\left[\begin{array}{ccc}
1 & 0 & l_{1}+l_{7} \\
0 & 1 & 0 \\
0 & 0 & 1
\end{array}\right]
$$

Then the homogeneous form of the forward kinematic map is

$$
T(\phi)=\left[\begin{array}{ccc}
\cos \left(\phi_{1}+\phi_{7}+\phi_{9}\right) & -\sin \left(\phi_{1}+\phi_{7}+\phi_{9}\right) & l_{1} \cos \phi_{1}+l_{7} \cos \left(\phi_{1}+\phi_{7}\right) \\
\sin \left(\phi_{1}+\phi_{7}+\phi_{9}\right) & \cos \left(\phi_{1}+\phi_{7}+\phi_{9}\right) & l_{1} \sin \phi_{1}+l_{7} \sin \left(\phi_{1}+\phi_{7}\right) \\
0 & 0 & 1
\end{array}\right]
$$

This can be more simply expressed in terms of link rotation variables using $\theta_{1}=\phi_{1}, \theta_{7}=\phi_{1}+\phi_{7}$, and $\theta_{9}=\phi_{1}+\phi_{7}+\phi_{9}$. Moreover, for purposes of singularity analysis it is preferable to work with a local representation of the kinematic mapping $T$. Simply using the angle $\theta_{9}$ to parametrise the rotation matrix that constitutes the top left $(2 \times 2)$ block of the homogeneous transformation, the local representation is

$$
\left(\theta_{1}, \theta_{7}, \theta_{9}\right) \mapsto\left[\begin{array}{c}
\theta_{9} \\
l_{1} \cos \theta_{1}+l_{7} \cos \theta_{7} \\
l_{1} \sin \theta_{1}+l_{7} \sin \theta_{7}
\end{array}\right]
$$

In this form, we have simply made use of the passive variables that describe the kinematics of the underlying $3 \mathrm{R}$ mechanism. These can now be expressed using (8.11) in terms of the input variables. Hence, the kinematic mapping for the GM can be expressed as a function $f: \mathbb{R}^{3} \rightarrow \mathbb{R}^{3}$, where

$$
f\left(\theta_{1}, \theta_{2}, \theta_{3}\right)=\left[\begin{array}{c}
\beta_{1} \theta_{1}+\beta_{2} \theta_{2}+\beta_{3} \theta_{3} \\
l_{1} \cos \theta_{1}+l_{7} \cos \left(\alpha_{1} \theta_{1}+\alpha_{3} \theta_{3}\right) \\
l_{1} \sin \theta_{1}+l_{7} \sin \left(\alpha_{1} \theta_{1}+\alpha_{3} \theta_{3}\right)
\end{array}\right]
$$

It is worth noting that by judicious choice of gear ratios the rotation of the 
end-effector can be made independent of one or more input variables. For example, setting $\rho_{1} \rho_{3}=\rho_{5} \rho_{6}=1$ (equivalently $r_{2}=r_{6}=r_{9}$ ) ensures the rotation is independent of $\theta_{1}, \theta_{3}$ and is directly equal to $\theta_{2}$.

Finally, to find singularities we need to investigate the Jacobian of the kinematic mapping $f$. From (8.25) we obtain

$$
J=\left[\begin{array}{ccc}
\beta_{1} & \beta_{2} & \beta_{3} \\
-l_{1} \sin \theta_{1}-\alpha_{1} l_{7} \sin \left(\alpha_{1} \theta_{1}+\alpha_{3} \theta_{3}\right) & 0 & -\alpha_{3} l_{7} \sin \left(\alpha_{1} \theta_{1}+\alpha_{3} \theta_{3}\right) \\
l_{1} \cos \theta_{1}-\alpha_{1} l_{7} \cos \left(\alpha_{1} \theta_{1}+\alpha_{3} \theta_{3}\right) & 0 & \alpha_{3} l_{7} \cos \left(\alpha_{1} \theta_{1}+\alpha_{3} \theta_{3}\right)
\end{array}\right]
$$

For a singularity, we require

$$
\operatorname{det} J=\alpha_{3} \beta_{2} l_{1} l_{7} \sin \left(\theta_{1}-\alpha_{1} \theta_{1}-\alpha_{3} \theta_{3}\right)=0
$$

The design parameters $\alpha_{3}, \beta_{2}, l_{1}, l_{7}$ are assumed non-zero so the GM is singular if and only if $\sin \left(\theta_{1}-\alpha_{1} \theta_{1}-\alpha_{3} \theta_{3}\right)=0$ and hence

$$
\theta_{1}-\theta_{3}=\frac{\eta_{3} \pi}{\rho_{2} \rho_{4}} \quad \eta_{3} \in \mathbb{Z}
$$

We note that, of course, this corresponds precisely to the output map singularities found in (8.19).

Thus, the singular configurations of mechanism in Figure 8.1 are strictly contingent on the difference between input variables $\theta_{1}$ and $\theta_{3}$. It can be concluded that increasing the product of gear ratios $\rho_{2} \rho_{4}$ connecting gear wheels $L_{3}$ and $L_{7}$ can cause more singular points in the joint space, while keeping it close to zero will reduce singularities. It must be noticed that having $\rho_{2} \rho_{4} \ll 1$ may have dynamic consequences. The images of the singularity set in the workspace of course correspond to the expected singular configurations in which carrier arms $L_{1}$ and $L_{7}$ are collinear. 


\section{Chapter 9}

\section{Spatial parallel manipulators}

Having demonstrated the application of the constraint-based approach across a range of planar manipulators, the next goal is to test its effectiveness for spatial parallel manipulators (SPMs). A number of challenges have to be admitted. Firstly, a link in space requires at least six pose variables as opposed to three in the planar case. In effect, to obtain a single global description, it may be preferable to work with dual quaternions, involving eight variables subject to two additional constraints. Secondly, SPM typically require more links than their planar counterparts. For example, the natural extension of the 3-RPR PPM is the (general) Gough-Stewart (GS) platform [100], which may be interpreted as a 6-UPS (or sometimes 6-SPS) SPM.

Finally, as well as a great number of links, there are more joints and hence a larger number of constraint equations. In the case of the GS platform, there are 13 moving links requiring 78 (or 104 using dual quaternions) pose variables and, for the 6 -SPS version, $6 \times 11=66$ (or $66+22=92)$ constraint equations. Six of the resulting degrees of freedom corresponding to legs rotating about the prismatic joint. As a result, the Jacobian is very large and the number of minors required to vanish is intractable.

Nevertheless, the analysis is attempted for a simpler mechanism, with the aim of finding some patterns that may indicate a way to proceed in more 
complex cases.

\subsection{Spatial 3-RPS parallel manipulator}

A general spatial 3-RPS PM is illustrated in Figure 9.1, along with a choice of moving and reference frames, together with design parameters. This mechanism is made up of $k=7$ moving components. Using dual quaternions in (2.33) to represent spatial displacements, there are totally 56 pose variables required to express displacement of the whole mechanism in the ambient space. In addition, there are totally 18 design parameters

- the base: $b_{2}, b_{3}, \beta$

- offset distances: $d_{1}, d_{2}, d_{3}$

- angles between joint axes: $\psi_{1}, \ldots, \psi_{6}$

- link lengths: $a_{4}, a_{5}, a_{6}$

- the platform: $a_{8}, a_{9}, \alpha$

This spatial PM consists of three R-joints, three P-joints, and three S-joints. As shown in Figure 9.1, the body coordinates of these joints in the preceding and following frames are

$$
\begin{array}{lrr}
\mathbf{r}_{i}^{-}=\left(0,-\sin \psi_{i}, \cos \psi_{i}\right)^{T}, & \mathbf{r}_{i}^{+}=(0,0,1)^{T} & i=1, \ldots, 6 \\
\mathbf{p}_{j}^{-}=\left(a_{j}, 0,0\right)^{T}, & \mathbf{p}_{j}^{+}=(0,0,0)^{T} & \\
\mathbf{n}_{j}^{-}=\left(0, \cos \psi_{j}, \sin \psi_{j}\right)^{T}, & \mathbf{n}_{j}^{+}=(0,1,0)^{T} & j=4,5,6 \\
\mathbf{p}_{k}^{+}=\left(0,0,-d_{k}\right)^{T} & k=1,2,3 & \\
\mathbf{p}_{1}^{R}=(0,0,0)^{T}, & \mathbf{p}_{2}^{R}=\left(b_{2}, 0,0\right)^{T}, & \mathbf{p}_{3}^{R}=\left(b_{3} \cos \beta, b_{3} \sin \beta, 0\right)^{T} \\
\mathbf{p}_{7}^{7}=(0,0,0)^{T}, & \mathbf{p}_{8}^{7}=\left(a_{8}, 0,0\right)^{T}, & \mathbf{p}_{9}^{7}=\left(a_{9} \cos \alpha, b_{3} \sin \alpha, 0\right)^{T} \\
\mathbf{p}_{7}^{4}=\mathbf{p}_{8}^{5}=\mathbf{p}_{9}^{6}=(0,0,0)^{T} & &
\end{array}
$$




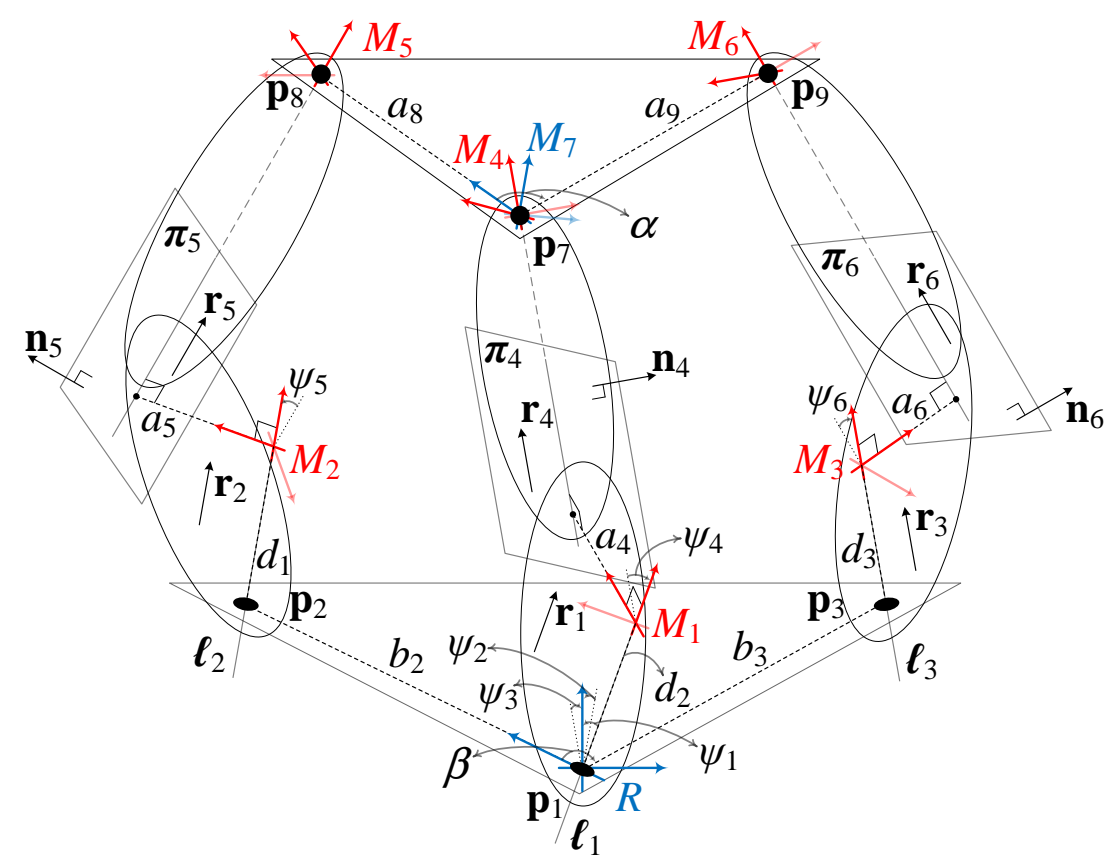

Figure 9.1: 3-RPS spatial PM with associated moving frames and design parameters

Each R-, P-, and S-joint imposes a set of constraint equations of the form (A.1), (A.2), (A.5), respectively. Every R- and P-joint imposes five constraint equations while every S-joint imposes three. Since a dual quaternions is employed to represent an element of $S E(3)$, there are two extra pose variables and conditions (2.38a), (2.38b) corresponding to each mobile link. 


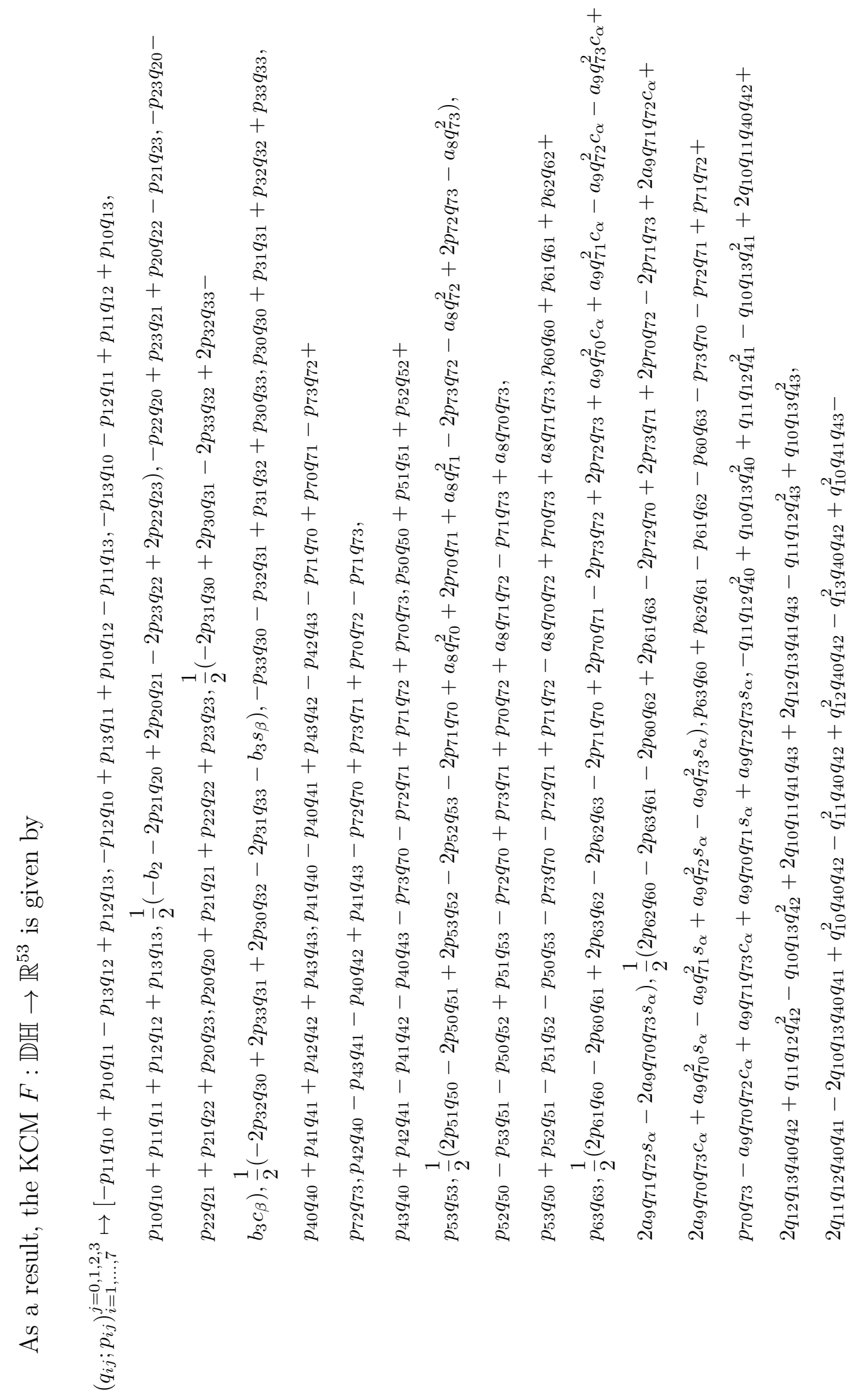




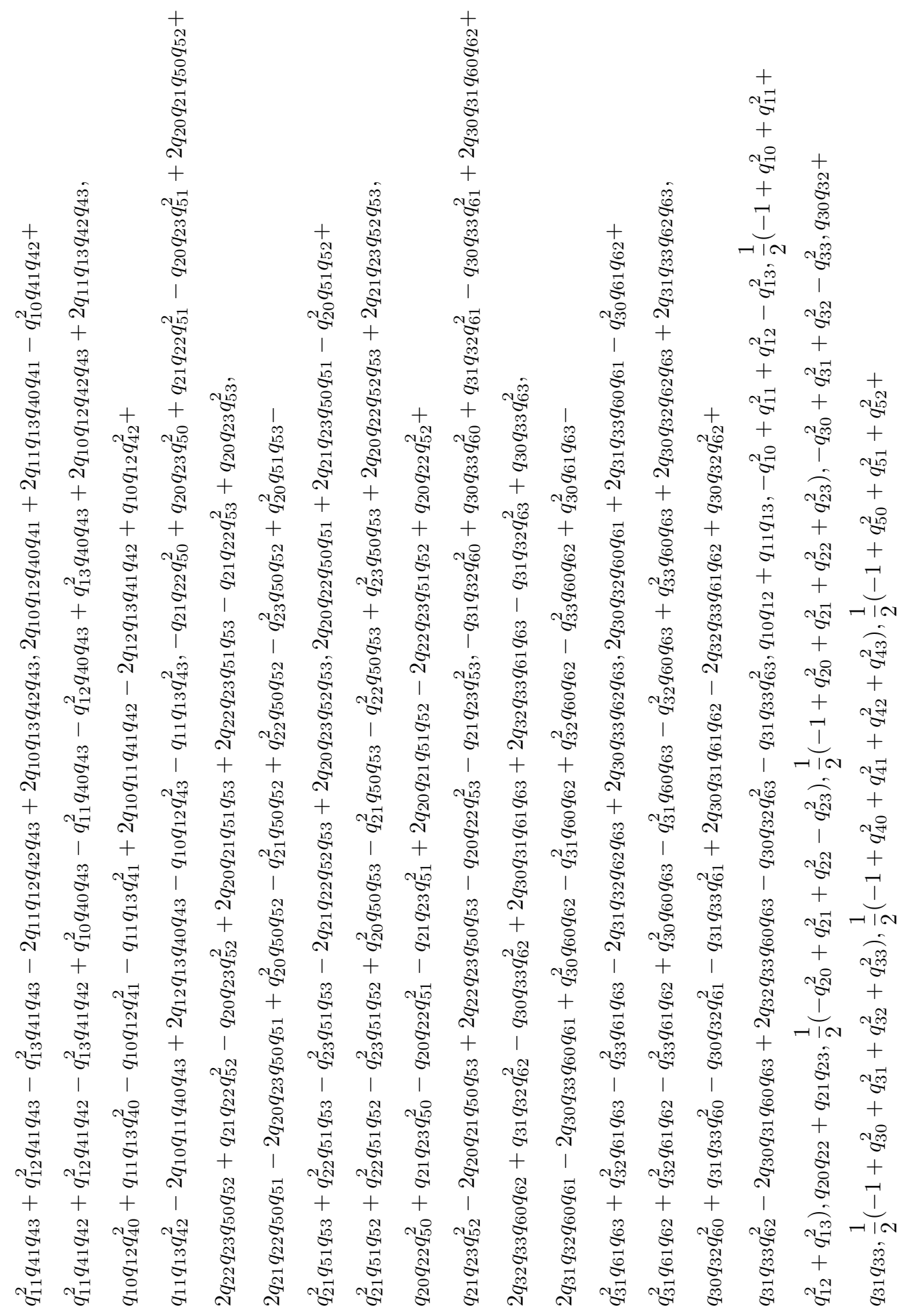




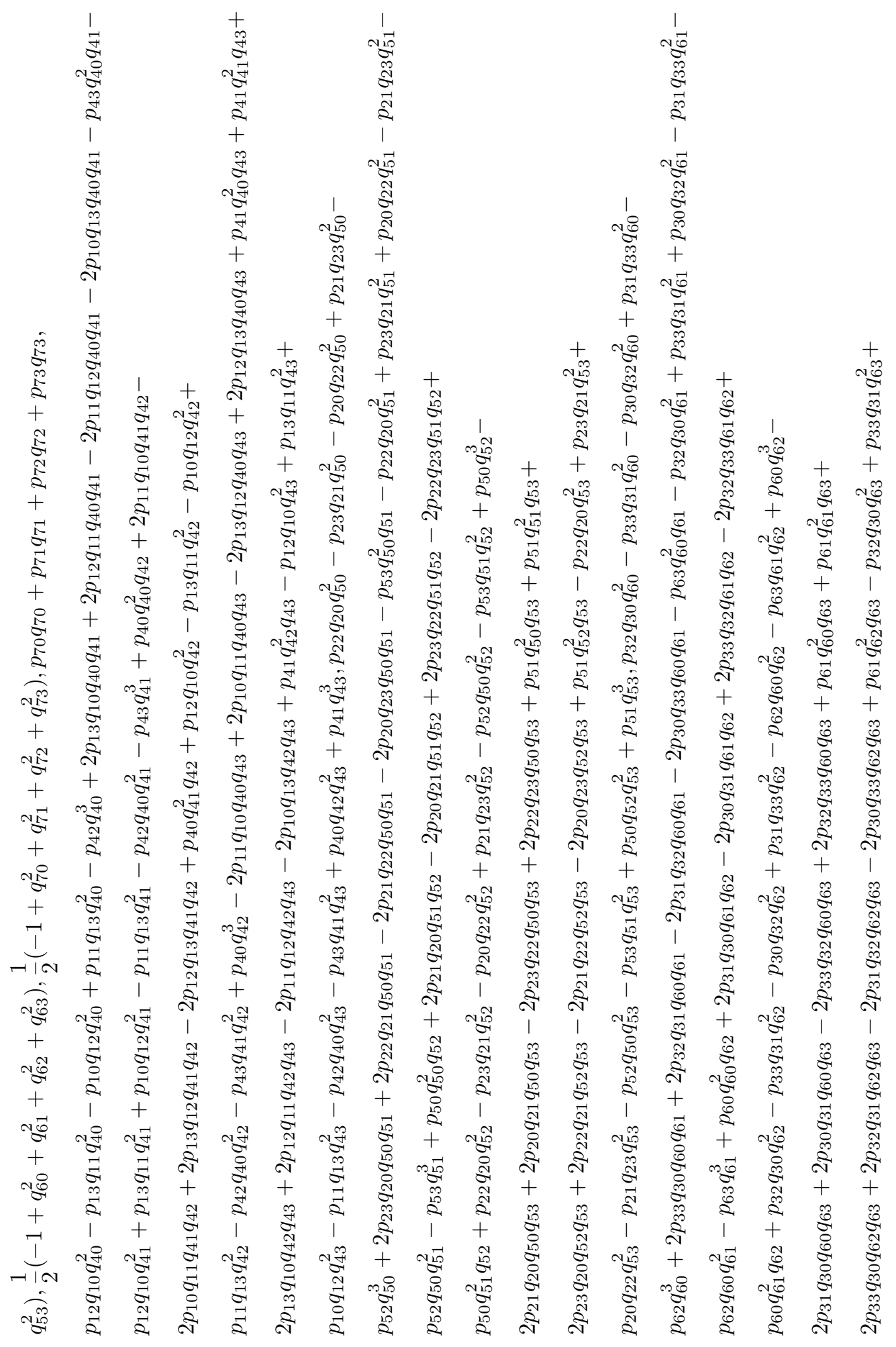


$\stackrel{\overparen{9}}{0}$

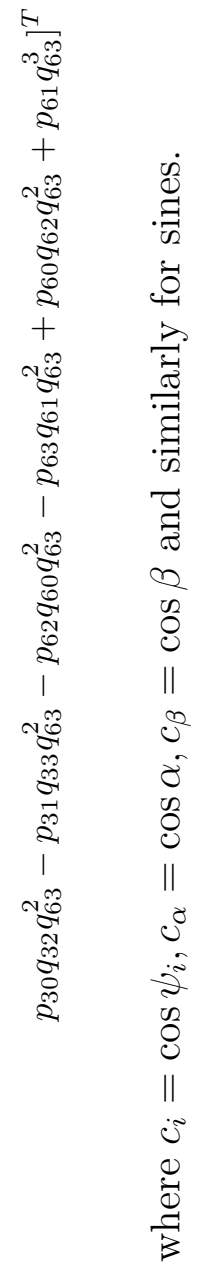


The KCM in (9.1) specifies the D-space for the full family of 3-RPS SPMs with design parameters. However in the literature [118, 119], a specific architecture has been widely studied, with the following specific choices of design parameters

- axes of three R-joints coplanar: $d_{i}=0, \psi_{i}=\pi / 2, i=1,2,3$

- in each leg, the P-joint is perpendicular to and passes through the R-joint: $a_{i}=0, \psi_{i}=\pi / 2, i=4,5,6$

An additional assumption is that the three RP intersect and the three Sjoints form an equilateral triangle that does not have a significant impact on the analysis.

These assumptions simplify the KCM and so the Jacobian, which is a $(53 \times 56)$ matrix, can be partitioned

$$
J F=\left[\begin{array}{ll}
A & C \\
B & D
\end{array}\right]
$$

where $A, B, C, D$ are $(24 \times 24),(29 \times 24),(24 \times 32),(29 \times 32)$ block matrices, respectively. A MATHEMATICA worksheet detailing the computation can be found here [110]. A summary is as follows.

Further, block matrix $A$ consists of six diagonal $(4 \times 4)$ block submatrices

$$
\left[\begin{array}{cccccc}
A_{11} & 0_{4} & 0_{4} & 0_{4} & 0_{4} & 0_{4} \\
0_{4} & A_{22} & 0_{4} & 0_{4} & 0_{4} & 0_{4} \\
0_{4} & 0_{4} & A_{33} & 0_{4} & 0_{4} & 0_{4} \\
0_{4} & 0_{4} & 0_{4} & A_{44} & 0_{4} & 0_{4} \\
0_{4} & 0_{4} & 0_{4} & 0_{4} & A_{55} & 0_{4} \\
0_{4} & 0_{4} & 0_{4} & 0_{4} & 0_{4} & A_{66}
\end{array}\right]
$$


where

$$
A_{i i}=\left[\begin{array}{cccc}
q_{i 1} & -q_{i 0} & q_{i 3} & -q_{i 2} \\
q_{i 2} & -q_{i 3} & -q_{i 0} & q_{i 1} \\
q_{i 3} & q_{i 2} & -q_{i 1} & -q_{i 0} \\
q_{i 0} & q_{i 1} & q_{i 2} & q_{i 3}
\end{array}\right] \quad i=1, \ldots, 6
$$

Block matrix $B$ is of the form

$$
\left[\begin{array}{cc}
0_{26 \times 12} & 0_{26 \times 12} \\
B_{21} & B_{22}
\end{array}\right]
$$

where $B_{2 j}$ is $(3 \times 12), j=1,2$ with

$$
B_{21}=\left[\begin{array}{ccc}
\mathbf{b}_{27}^{T} & \mathbf{0}^{T} & \mathbf{0}^{T} \\
\mathbf{0}^{T} & \mathbf{b}_{28}^{T} & \mathbf{0}^{T} \\
\mathbf{0}^{T} & \mathbf{0}^{T} & \mathbf{b}_{29}^{T}
\end{array}\right]
$$

where $\mathbf{0}^{T}=(0,0,0,0)^{T}$ is a row vector.

$$
\begin{aligned}
\mathbf{b}_{27}^{T}= & -2\left(q_{13} q_{40} q_{41}+q_{11} q_{41} q_{42}-q_{11} q_{40} q_{43}+q_{13} q_{42} q_{43}\right)+q_{12}\left(-q_{40}^{2}+q_{41}^{2}-q_{42}^{2}+q_{43}^{2}\right), \\
& -2\left(q_{12} q_{40} q_{41}-q_{10} q_{41} q_{42}+q_{10} q_{40} q_{43}+q_{12} q_{42} q_{43}\right)+q_{13}\left(q_{40}^{2}-q_{41}^{2}+q_{42}^{2}-q_{43}^{2}\right), \\
& 2\left(q_{11} q_{40} q_{41}-q_{13} q_{41} q_{42}+q_{13} q_{40} q_{43}+q_{11} q_{42} q_{43}\right)+q_{10}\left(q_{40}^{2}-q_{41}^{2}+q_{42}^{2}-q_{43}^{2}\right), \\
& \left.2\left(q_{10} q_{40} q_{41}+q_{12} q_{41} q_{42}-q_{12} q_{40} q_{43}+q_{10} q_{42} q_{43}\right)+q_{11}\left(-q_{40}^{2}+q_{41}^{2}-q_{42}^{2}+q_{43}^{2}\right)\right]^{T}, \\
\mathbf{b}_{28}^{T}=[ & -2\left(q_{23} q_{50} q_{51}+q_{21} q_{51} q_{52}-q_{21} q_{50} q_{53}+q_{23} q_{52} q_{53}\right)+q_{22}\left(-q_{50}^{2}+q_{51}^{2}-q_{52}^{2}+q_{53}^{2}\right), \\
& -2\left(q_{22} q_{50} q_{51}-q_{20} q_{51} q_{52}+q_{20} q_{50} q_{53}+q_{22} q_{52} q_{53}\right)+q_{23}\left(q_{50}^{2}-q_{51}^{2}+q_{52}^{2}-q_{53}^{2}\right), \\
& 2\left(q_{21} q_{50} q_{51}-q_{23} q_{51} q_{52}+q_{23} q_{50} q_{53}+q_{21} q_{52} q_{53}\right)+q_{20}\left(q_{50}^{2}-q_{51}^{2}+q_{52}^{2}-q_{53}^{2}\right), \\
& \left.2\left(q_{20} q_{50} q_{51}+q_{22} q_{51} q_{52}-q_{22} q_{50} q_{53}+q_{20} q_{52} q_{53}\right)+q_{21}\left(-q_{50}^{2}+q_{51}^{2}-q_{52}^{2}+q_{53}^{2}\right)\right]^{T}, \\
\mathbf{b}_{29}^{T}= & -2\left(q_{33} q_{60} q_{61}+q_{31} q_{61} q_{62}-q_{31} q_{60} q_{63}+q_{33} q_{62} q_{63}\right)+q_{32}\left(-q_{60}^{2}+q_{61}^{2}-q_{62}^{2}+q_{63}^{2}\right), \\
& -2\left(q_{32} q_{60} q_{61}-q_{30} q_{61} q_{62}+q_{30} q_{60} q_{63}+q_{32} q_{62} q_{63}\right)+q_{33}\left(q_{60}^{2}-q_{61}^{2}+q_{62}^{2}-q_{63}^{2}\right), \\
& 2\left(q_{31} q_{60} q_{61}-q_{33} q_{61} q_{62}+q_{33} q_{60} q_{63}+q_{31} q_{62} q_{63}\right)+q_{30}\left(q_{60}^{2}-q_{61}^{2}+q_{62}^{2}-q_{63}^{2}\right), \\
& \left.2\left(q_{30} q_{60} q_{61}+q_{32} q_{61} q_{62}-q_{32} q_{60} q_{63}+q_{30} q_{62} q_{63}\right)+q_{31}\left(-q_{60}^{2}+q_{61}^{2}-q_{62}^{2}+q_{63}^{2}\right)\right]^{T}
\end{aligned}
$$


and

$$
B_{22}=\left[\begin{array}{cccccccccccc}
q_{42} & q_{43} & -q_{40} & -q_{41} & 0 & 0 & 0 & 0 & 0 & 0 & 0 & 0 \\
0 & 0 & 0 & 0 & q_{52} & q_{53} & -q_{50} & -q_{51} & 0 & 0 & 0 & 0 \\
0 & 0 & 0 & 0 & 0 & 0 & 0 & 0 & q_{62} & q_{63} & -q_{60} & -q_{61}
\end{array}\right]
$$

Block matrix $C$ has shape

$$
\left[\begin{array}{cccccccc}
C_{11} & 0_{4} & 0_{4} & 0_{4} & 0_{4} & 0_{4} & 0_{4} & 0_{4} \\
0_{4} & C_{22} & 0_{4} & 0_{4} & 0_{4} & 0_{4} & 0_{4} & 0_{4} \\
0_{4} & 0_{4} & C_{33} & 0_{4} & 0_{4} & 0_{4} & 0_{4} & 0_{4} \\
0_{4} & 0_{4} & 0_{4} & C_{44} & 0_{4} & 0_{4} & C_{47} & C_{48} \\
0_{4} & 0_{4} & 0_{4} & 0_{4} & C_{55} & 0_{4} & C_{57} & C_{58} \\
0_{4} & 0_{4} & 0_{4} & 0_{4} & 0_{4} & C_{66} & C_{67} & C_{68}
\end{array}\right]
$$

with each $C_{i, j}$ and $0_{4}$ all $(4 \times 4)$ blocks and 


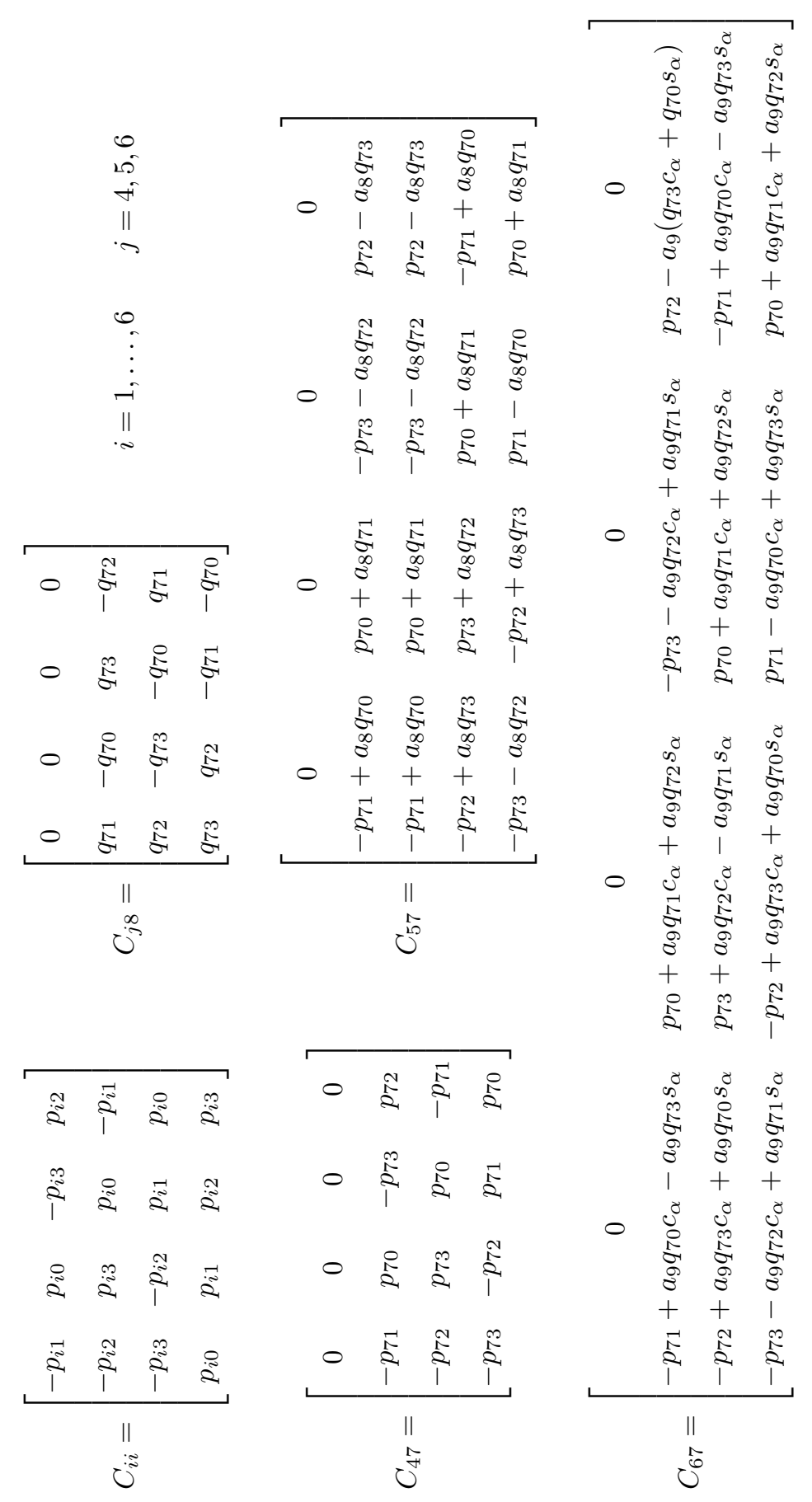


Finally, Block $D$ is given by

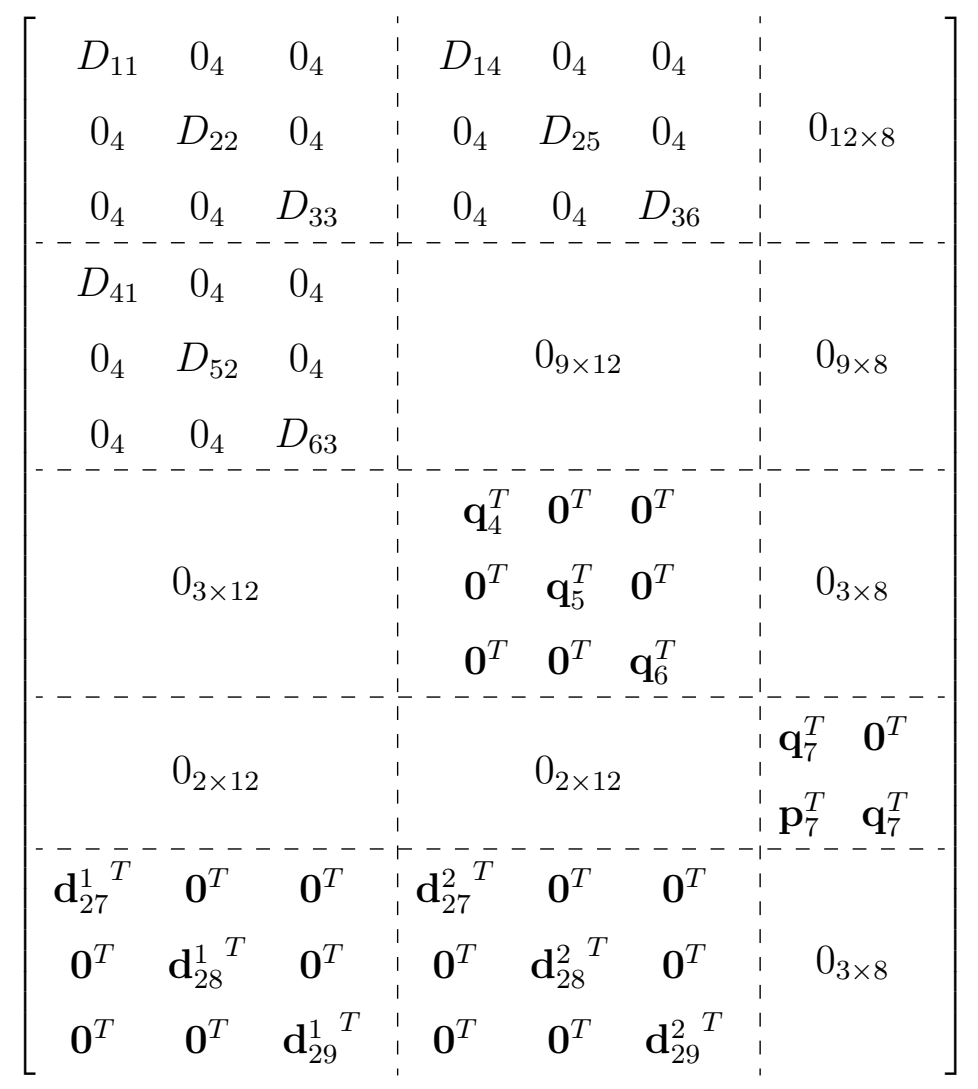

where $\mathbf{q}_{i}^{T}=\left(q_{i 0}, q_{i 1}, q_{i 2}, q_{i 3}\right), i=4, \ldots, 7, \mathbf{p}_{7}^{T}=\left(p_{70}, p_{71}, p_{72}, p_{73}\right)$ and

$$
\begin{aligned}
\mathbf{d}_{27}^{1}{ }^{T} & =\left[2\left(p_{13} q_{40} q_{41}+p_{11} q_{41} q_{42}-p_{11} q_{40} q_{43}+p_{13} q_{42} q_{43}\right)+p_{12}\left(q_{40}^{2}-q_{41}^{2}+q_{42}^{2}-q_{43}^{2}\right),\right. \\
& 2\left(p_{12} q_{40} q_{41}-p_{10} q_{41} q_{42}+p_{10} q_{40} q_{43}+p_{12} q_{42} q_{43}\right)+p_{13}\left(-q_{40}^{2}+q_{41}^{2}-q_{42}^{2}+q_{43}^{2}\right), \\
& -2\left(p_{11} q_{40} q_{41}-p_{13} q_{41} q_{42}+p_{13} q_{40} q_{43}+p_{11} q_{42} q_{43}\right)+p_{10}\left(-q_{40}^{2}+q_{41}^{2}-q_{42}^{2}+q_{43}^{2}\right), \\
& \left.-2\left(p_{10} q_{40} q_{41}+p_{12} q_{41} q_{42}-p_{12} q_{40} q_{43}+p_{10} q_{42} q_{43}\right)+p_{11}\left(q_{40}^{2}-q_{41}^{2}+q_{42}^{2}-q_{43}^{2}\right)\right]^{T}, \\
\mathbf{d}_{27}^{2}{ }^{T}= & {\left[-2 p_{13} q_{11} q_{40}-2 p_{10} q_{12} q_{40}+2 p_{11} q_{13} q_{40}-3 p_{42} q_{40}^{2}+2 p_{13} q_{10} q_{41}-2 p_{11} q_{12} q_{41}\right.} \\
& -2 p_{10} q_{13} q_{41}-2 p_{43} q_{40} q_{41}-p_{42} q_{41}^{2}+2 p_{40} q_{40} q_{42}-p_{42} q_{42}^{2}+2\left(-p_{11} q_{10}+p_{10} q_{11}\right. \\
& \left.-p_{13} q_{12}+p_{41} q_{40}\right) q_{43}-p_{42} q_{43}^{2}+2 p_{12}\left(q_{10} q_{40}+q_{11} q_{41}+q_{13} q_{43}\right),-2 p_{11} q_{12} q_{40} \\
& -2 p_{10} q_{13} q_{40}-p_{43} q_{40}^{2}+2 p_{10} q_{12} q_{41}-2 p_{11} q_{13} q_{41}-2 p_{42} q_{40} q_{41}-3 p_{43} q_{41}^{2} \\
& +2 p_{11} q_{10} q_{42}-2 p_{10} q_{11} q_{42}+2 p_{40} q_{41} q_{42}-p_{43} q_{42}^{2}+2 p_{13}\left(q_{10} q_{40}+q_{11} q_{41}+q_{12} q_{42}\right) \\
& +2 p_{12}\left(q_{11} q_{40}-q_{10} q_{41}-q_{13} q_{42}\right)+2 p_{41} q_{41} q_{43}-p_{43} q_{43}^{2}, 2\left(p_{11} q_{10}-p_{10} q_{11}+p_{13} q_{12}\right. \\
& \left.-p_{12} q_{13}\right) q_{41}-2\left(-p_{12} q_{10}+p_{13} q_{11}+p_{10} q_{12}-p_{11} q_{13}+p_{42} q_{40}+p_{43} q_{41}\right) q_{42} \\
& +2\left(p_{13} q_{10}+p_{12} q_{11}-p_{11} q_{12}-p_{10} q_{13}+p_{41} q_{42}\right) q_{43}+p_{40}\left(q_{40}^{2}+q_{41}^{2}+3 q_{42}^{2}+q_{43}^{2}\right),
\end{aligned}
$$




$$
\begin{aligned}
& -2 p_{13} q_{12} q_{40}+2 p_{12} q_{13} q_{40}+p_{41} q_{40}^{2}+p_{41} q_{41}^{2}+2 p_{13} q_{10} q_{42}+2 p_{12} q_{11} q_{42}+p_{41} q_{42}^{2} \\
& -2\left(p_{12} q_{10}-p_{13} q_{11}+p_{42} q_{40}+p_{43} q_{41}-p_{40} q_{42}\right) q_{43}+3 p_{41} q_{43}^{2}+2 p_{10}\left(q_{11} q_{40}\right. \\
& \left.\left.-q_{13} q_{42}+q_{12} q_{43}\right)-2 p_{11}\left(q_{10} q_{40}+q_{12} q_{42}+q_{13} q_{43}\right)\right]^{T}, \\
& \mathbf{d}_{28}^{1}{ }^{T}=\left[2\left(p_{23} q_{50} q_{51}+p_{21} q_{51} q_{52}-p_{21} q_{50} q_{53}+p_{23} q_{52} q_{53}\right)+p_{22}\left(q_{50}^{2}-q_{51}^{2}+q_{52}^{2}-q_{53}^{2}\right)\right. \text {, } \\
& 2\left(p_{22} q_{50} q_{51}-p_{20} q_{51} q_{52}+p_{20} q_{50} q_{53}+p_{22} q_{52} q_{53}\right)+p_{23}\left(-q_{50}^{2}+q_{51}^{2}-q_{52}^{2}+q_{53}^{2}\right), \\
& -2\left(p_{21} q_{50} q_{51}-p_{23} q_{51} q_{52}+p_{23} q_{50} q_{53}+p_{21} q_{52} q_{53}\right)+p_{20}\left(-q_{50}^{2}+q_{51}^{2}-q_{52}^{2}+q_{53}^{2}\right), \\
& \left.-2\left(p_{20} q_{50} q_{51}+p_{22} q_{51} q_{52}-p_{22} q_{50} q_{53}+p_{20} q_{52} q_{53}\right)+p_{21}\left(q_{50}^{2}-q_{51}^{2}+q_{52}^{2}-q_{53}^{2}\right)\right]^{T}, \\
& \mathbf{d}_{28}^{2}{ }^{T}=\left[-2 p_{23} q_{21} q_{50}-2 p_{20} q_{22} q_{50}+2 p_{21} q_{23} q_{50}-3 p_{52} q_{50}^{2}+2 p_{23} q_{20} q_{51}-2 p_{21} q_{22} q_{51}\right. \\
& -2 p_{20} q_{23} q_{51}-2 p_{53} q_{50} q_{51}-p_{52} q_{51}^{2}+2 p_{50} q_{50} q_{52}-p_{52} q_{52}^{2}+2\left(-p_{21} q_{20}+p_{20} q_{21}\right. \\
& \left.-p_{23} q_{22}+p_{51} q_{50}\right) q_{53}-p_{52} q_{53}^{2}+2 p_{22}\left(q_{20} q_{50}+q_{21} q_{51}+q_{23} q_{53}\right),-2 p_{21} q_{22} q_{50} \\
& -2 p_{20} q_{23} q_{50}-p_{53} q_{50}^{2}+2 p_{20} q_{22} q_{51}-2 p_{21} q_{23} q_{51}-2 p_{52} q_{50} q_{51}-3 p_{53} q_{51}^{2} \\
& +2 p_{21} q_{20} q_{52}-2 p_{20} q_{21} q_{52}+2 p_{50} q_{51} q_{52}-p_{53} q_{52}^{2}+2 p_{23}\left(q_{20} q_{50}+q_{21} q_{51}+q_{22} q_{52}\right) \\
& +2 p_{22}\left(q_{21} q_{50}-q_{20} q_{51}-q_{23} q_{52}\right)+2 p_{51} q_{51} q_{53}-p_{53} q_{53}^{2}, 2\left(p_{21} q_{20}-p_{20} q_{21}\right. \\
& \left.+p_{23} q_{22}-p_{22} q_{23}\right) q_{51}-2\left(-p_{22} q_{20}+p_{23} q_{21}+p_{20} q_{22}-p_{21} q_{23}+p_{52} q_{50}+p_{53} q_{51}\right) q_{52} \\
& +2\left(p_{23} q_{20}+p_{22} q_{21}-p_{21} q_{22}-p_{20} q_{23}+p_{51} q_{52}\right) q_{53}+p_{50}\left(q_{50}^{2}+q_{51}^{2}+3 q_{52}^{2}+q_{53}^{2}\right), \\
& -2 p_{23} q_{22} q_{50}+2 p_{22} q_{23} q_{50}+p_{51} q_{50}^{2}+p_{51} q_{51}^{2}+2 p_{23} q_{20} q_{52}+2 p_{22} q_{21} q_{52}+p_{51} q_{52}^{2} \\
& -2\left(p_{22} q_{20}-p_{23} q_{21}+p_{52} q_{50}+p_{53} q_{51}-p_{50} q_{52}\right) q_{53}+3 p_{51} q_{53}^{2}+2 p_{20}\left(q_{21} q_{50}-q_{23} q_{52}\right. \\
& \left.\left.+q_{22} q_{53}\right)-2 p_{21}\left(q_{20} q_{50}+q_{22} q_{52}+q_{23} q_{53}\right)\right]^{T}, \\
& \mathbf{d}_{29}^{1}{ }^{T}=\left[2\left(p_{33} q_{60} q_{61}+p_{31} q_{61} q_{62}-p_{31} q_{60} q_{63}+p_{33} q_{62} q_{63}\right)+p_{32}\left(q_{60}^{2}-q_{61}^{2}+q_{62}^{2}-q_{63}^{2}\right)\right. \text {, } \\
& 2\left(p_{32} q_{60} q_{61}-p_{30} q_{61} q_{62}+p_{30} q_{60} q_{63}+p_{32} q_{62} q_{63}\right)+p_{33}\left(-q_{60}^{2}+q_{61}^{2}-q_{62}^{2}+q_{63}^{2}\right), \\
& -2\left(p_{31} q_{60} q_{61}-p_{33} q_{61} q_{62}+p_{33} q_{60} q_{63}+p_{31} q_{62} q_{63}\right)+p_{30}\left(-q_{60}^{2}+q_{61}^{2}-q_{62}^{2}+q_{63}^{2}\right), \\
& \left.-2\left(p_{30} q_{60} q_{61}+p_{32} q_{61} q_{62}-p_{32} q_{60} q_{63}+p_{30} q_{62} q_{63}\right)+p_{31}\left(q_{60}^{2}-q_{61}^{2}+q_{62}^{2}-q_{63}^{2}\right)\right]^{T}, \\
& \mathbf{d}_{29}^{2}{ }^{T}=\left[-2 p_{33} q_{31} q_{60}-2 p_{30} q_{32} q_{60}+2 p_{31} q_{33} q_{60}-3 p_{62} q_{60}^{2}+2 p_{33} q_{30} q_{61}-2 p_{31} q_{32} q_{61}\right. \\
& -2 p_{30} q_{33} q_{61}-2 p_{63} q_{60} q_{61}-p_{62} q_{61}^{2}+2 p_{60} q_{60} q_{62}-p_{62} q_{62}^{2}+2\left(-p_{31} q_{30}+p_{30} q_{31}\right. \\
& \left.-p_{33} q_{32}+p_{61} q_{60}\right) q_{63}-p_{62} q_{63}^{2}+2 p_{32}\left(q_{30} q_{60}+q_{31} q_{61}+q_{33} q_{63}\right),-2 p_{31} q_{32} q_{60} \\
& -2 p_{30} q_{33} q_{60}-p_{63} q_{60}^{2}+2 p_{30} q_{32} q_{61}-2 p_{31} q_{33} q_{61}-2 p_{62} q_{60} q_{61}-3 p_{63} q_{61}^{2} \\
& +2 p_{31} q_{30} q_{62}-2 p_{30} q_{31} q_{62}+2 p_{60} q_{61} q_{62}-p_{63} q_{62}^{2}+2 p_{33}\left(q_{30} q_{60}+q_{31} q_{61}+q_{32} q_{62}\right) \\
& +2 p_{32}\left(q_{31} q_{60}-q_{30} q_{61}-q_{33} q_{62}\right)+2 p_{61} q_{61} q_{63}-p_{63} q_{63}^{2}, 2\left(p_{31} q_{30}-p_{30} q_{31}+p_{33} q_{32}\right. \\
& \left.-p_{32} q_{33}\right) q_{61}-2\left(-p_{32} q_{30}+p_{33} q_{31}+p_{30} q_{32}-p_{31} q_{33}+p_{62} q_{60}+p_{63} q_{61}\right) q_{62} \\
& +2\left(p_{33} q_{30}+p_{32} q_{31}-p_{31} q_{32}-p_{30} q_{33}+p_{61} q_{62}\right) q_{63}+p_{60}\left(q_{60}^{2}+q_{61}^{2}+3 q_{62}^{2}+q_{63}^{2}\right),
\end{aligned}
$$




$$
\begin{aligned}
& -2 p_{33} q_{32} q_{60}+2 p_{32} q_{33} q_{60}+p_{61} q_{60}^{2}+p_{61} q_{61}^{2}+2 p_{33} q_{30} q_{62}+2 p_{32} q_{31} q_{62}+p_{61} q_{62}^{2} \\
& -2\left(p_{32} q_{30}-p_{33} q_{31}+p_{62} q_{60}+p_{63} q_{61}-p_{60} q_{62}\right) q_{63}+3 p_{61} q_{63}^{2}+2 p_{30}\left(q_{31} q_{60}-q_{33} q_{62}\right. \\
& \left.\left.+q_{32} q_{63}\right)-2 p_{31}\left(q_{30} q_{60}+q_{32} q_{62}+q_{33} q_{63}\right)\right]^{T}
\end{aligned}
$$




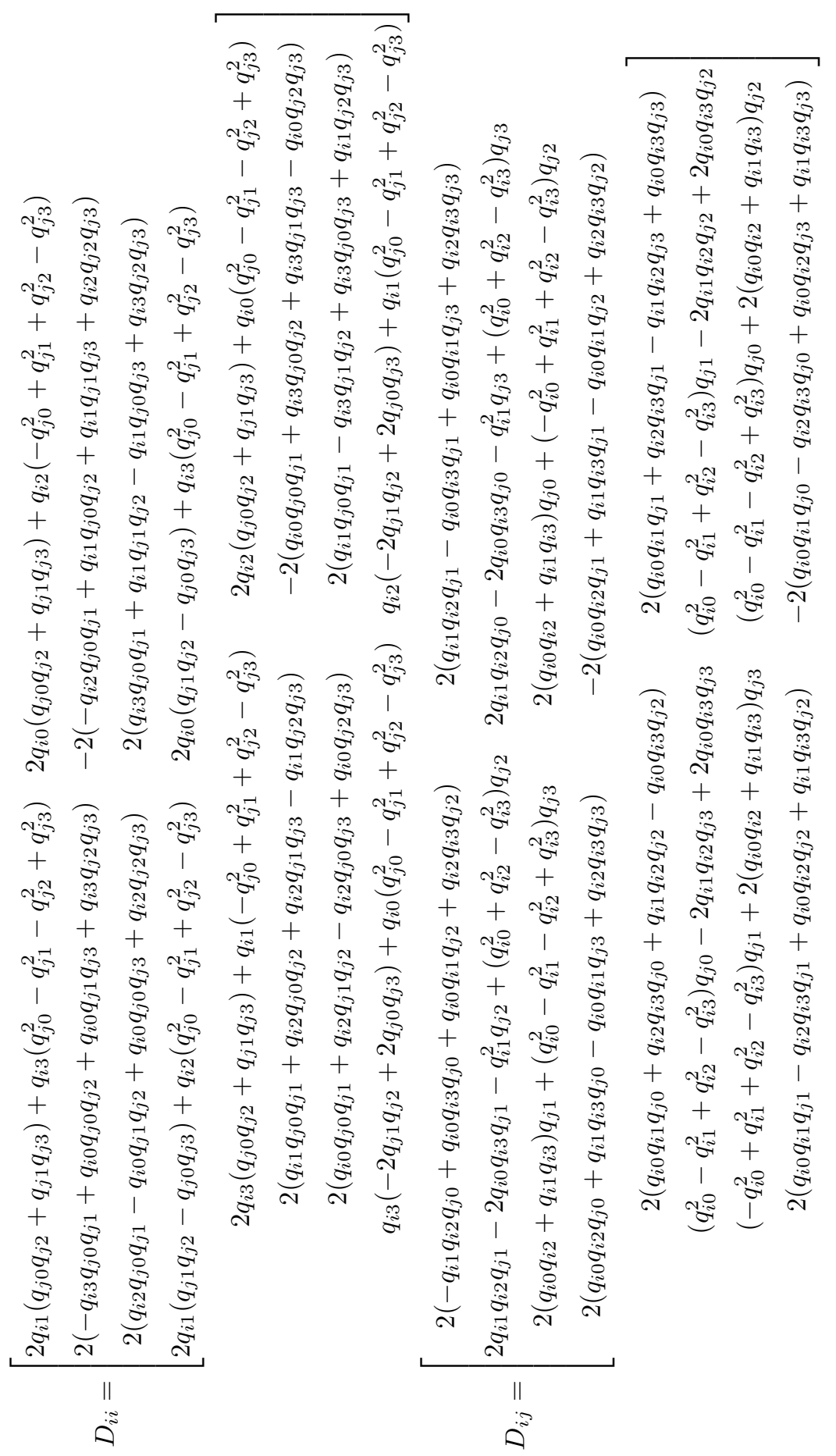


and

$$
D_{j i}=\left[\begin{array}{cccc}
q_{i 2} & q_{i 3} & q_{i 0} & q_{i 1} \\
-2 q_{i 0} & 2 q_{i 1} & 2 q_{i 2} & -2 q_{i 3} \\
q_{i 0} & q_{i 1} & q_{i 2} & q_{i 3}
\end{array}\right], \quad i=1,2,3, j=i+3
$$

Since $A$ is non-singular, the determinant of $J F$ is then given in terms of the Schur complement of $A$ by

$$
\operatorname{det} J F=\operatorname{det} A \operatorname{det}\left(D-B A^{-1} C\right)
$$

where, since $\operatorname{det} A_{i i}=\left(q_{i 0}^{2}+q_{i 1}^{2}+q_{i 2}^{2}+q_{i 3}^{2}\right)^{2}=1, i=1, \ldots, 6, \operatorname{det} A=1$. On the other hand, the Schur complement $M:=D-B A^{-1} C$ may be singular.

Indeed, we have a $\mathrm{C}$-space singularity whenever the $\mathrm{KCM}$ satisfies $F=\mathbf{0}$ and rank $<29$. After finding $A^{-1}$ and doing the rest of computation, the $(29 \times 32)$ matrix $M$ can be partitioned as

$$
\left[\begin{array}{cc}
M_{11} & 0_{24 \times 8} \\
M_{21} & M_{22}
\end{array}\right]
$$

where $M_{11}$ is a $(24 \times 24)$ square matrix, whose determinant factorises as

$$
\operatorname{det} M_{11}=m_{1} n_{1} m_{2} n_{2} m_{3} n_{3} m_{4} n_{4} m_{5} n_{5} m_{6} n_{6}
$$




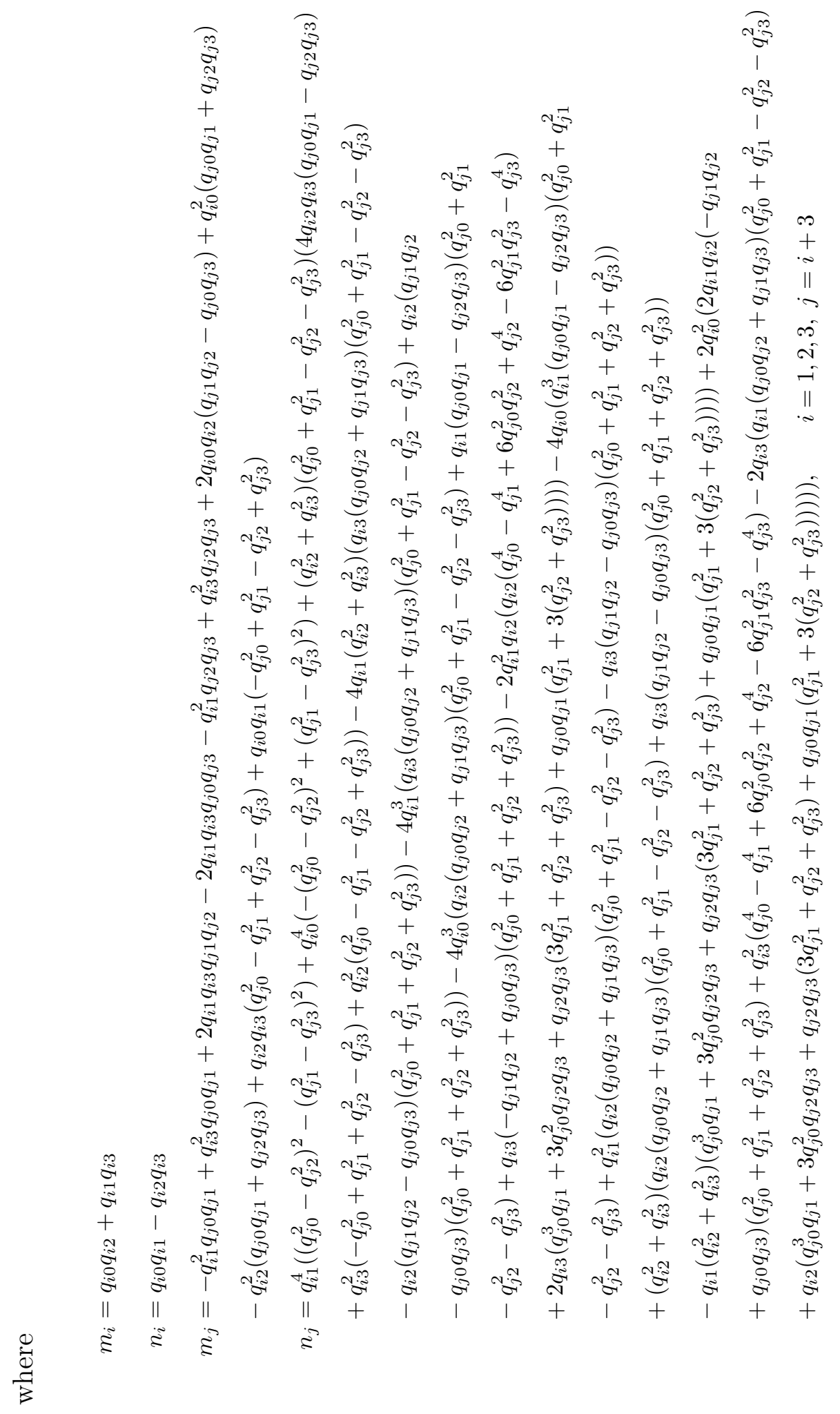


In principle, the C-space singularities correspond to any of these 12 factors vanishing together with $\operatorname{rank} M_{22}<5$ at any point in the $\mathrm{C}$-space. So far, explicit solutions to the resulting equations have not been computed. Nonetheless, it seems possible that relevant geometric conditions could be isolated. But finding Grashof-type conditions seems much more distant since the analysis here has been already restricted to a specific choice of design parameters. 


\section{Chapter 10}

\section{Conclusion}

In the robot kinematics literature, existing approaches to modelling manipulator kinematics and singularity analysis make use of combination of joint or input variables, pose or output variables and loop-closure equations. This approach has largely been successful for serial manipulators. However, it has been less effective for parallel manipulators. Joint variables measure the mobility between links. Whereas, pose variables measure positions and orientations of links, usually in some reference frame. Rather than regarding joints as allowing freedom between link, a complementary approach is to treat them as imposing constraints on the free motion of links, and thus, on their pose variables. This makes a constraint-based approach in terms of pose variables alone more desirable and even universal in that any (serial or parallel) manipulator can be represented as a collection of links and joints.

The core idea beyond the proposed approach in this thesis is to first remove all direct references to joint variables in the kinematic model and then define a global map (i.e. the KCM) just based on constraints. Moreover, we observe that apparent mobility constraints such as closed loops are in fact simple consequences of the joint constraints. Then, we can identify constraint singularities of the given mechanism by analysing the associated Jacobian of the $\mathrm{KCM}$. 
As noted in Chapter 1, most existing approaches analyse instantaneous configurations. This type of analysis (instantaneous analysis) is like taking different snapshots from the mechanism and working on them separately. The logic behind those approaches is to determine geometric conditions for singularity without computing the global set of singular points. The main drawback of an instantaneous analysis is that it may lose information by not computing the full configuration space.

A fundamental feature of our approach is to provide a reliable method for representing the configuration space, independent of the choice of input and output. The configuration space - as we define it - uniquely represents all possible pose variables of all moving links.

The proposed approach is theoretically robust. That is, the KCM is complete and generic because it fully captures the topological properties within a family of mechanisms. Although theoretically it can be utilised to study any constrained mechanical system, the approach has some limits in practice. For most spatial mechanisms, the large number of constraint equations and pose variables mean that the analysis faces computational difficulties. This could be a possible path in the future for investing whether a method of reduction based on algebraic geometry would be a candidate to overcome the complexity of a spatial mechanism.

A special feature of the proposed approach is that the impact of design parameters on mechanism singularities is examined more fundamentally. While some research has explored the impact of design parameters on kinematics, mostly it focuses on either specific choices or on generic cases. In this thesis, we have shown that configuration space singularities typically occur when the design parameters satisfy a single (i.e. codimension 1) condition, which was termed a Grashof-type condition. Existing Grashof-type conditions in the robot kinematics literature are for fairly simple mechanisms, such as the planar 4-R. This has been extended to more complex mechanisms such as 
the planar 3-RRR. As a future work, it would be interesting to study the geometry of these singularities more deeply to determine conditions for say, simple double points, cusps, tacnodes etc.

Recent research has started to identify the configuration space as the basic object for kinematic modelling and that inputs and outputs may be then selected in various ways. Therefore, the input and output mappings are defined from the C-space into relevant actuator and work spaces. In this thesis, this approach is explicitly defined in terms of pose variables. As a result, direct calculation of input and output singularities can be done. We show how this relates to the existence or otherwise of forward and inverse kinematic mappings. This approach seems to clarify the various existing classifications of manipulator singularities. However. fully connecting our results with, say, the classification of Zlatanov [2] would be worthwhile.

Several possible research directions have already been mentioned. The author would like to briefly mention a few more paths that could be taken in the future.

This thesis has focused on symbolic calculation and closed-form solutions. Nevertheless, as mentioned, this has shortcomings. It would be interesting to revisit some of the problems based on the numerical or interval analysis. Numerical and interval analysis approaches have been applied by Merlet [120] and Bohigas et al. [53]. It would be worthwhile to explore if they can be adapted to our model.

Kinematics and singularities of redundant manipulators is another direction to follow based on the proposed approach. In a regular robot manipulator number of inputs is equal to the degrees of freedom - all examples in this thesis are of this type. On the other hand, a redundant robot has greater number of inputs. This can help the robot to have a wider workspace and also to avoid singularities. One major impact will be the Jacobian of the input mapping will no longer be square. This is already consistent with our 
approach but it would be interesting to explore the impact on the dimensions of singularity sets via transversality.

Finally, dynamic analysis of manipulators may be adapted to this approach along the lines proposed by Park and Kim [54]. Given the precision of the kinematic model, there should be a real advantage in capturing the dynamics on the C-space since it can play a significant role in constructing control systems that are adapted for singularities [121]. 


\section{Appendix A}

\section{Constraint equations for spatial}

\section{joints}

Here, we list all spatial joints along with their constraint equations. The full detail can be found in [110].

1. R-joint:

$$
\begin{aligned}
& \left(q_{p^{i}} ; q_{d^{i}}\right)_{ \pm}^{i=0,1,2,3} \mapsto\left[\left(q_{p_{-}^{0}}^{2}\left(q_{p_{+}^{0}} q_{p_{+}^{2}}+q_{p_{+}^{1}} q_{p_{+}^{3}}\right)-q_{p_{-}^{1}}^{2}\left(q_{p_{+}^{0}} q_{p_{+}^{2}}+q_{p_{+}^{1}} q_{p_{+}^{3}}\right)\right.\right. \\
& -\left(q_{p_{-}^{2}}-q_{p_{-}^{3}}\right)\left(q_{p_{-}^{2}}+q_{p_{-}^{3}}\right)\left(q_{p_{+}^{0}} q_{p_{+}^{2}}+q_{p_{+}^{1}} q_{p_{+}^{3}}\right)+q_{p_{-}^{0}} q_{p_{-}^{2}}\left(-q_{p_{+}^{0}}^{2}\right. \\
& \left.\left.+q_{p_{+}^{1}}^{2}+q_{p_{+}^{2}}^{2}-q_{p_{+}^{3}}^{2}\right)+q_{p_{-}^{1}} q_{p_{-}^{3}}\left(-q_{p_{+}^{0}}^{2}+q_{p_{+}^{1}}^{2}+q_{p_{+}^{2}}^{2}-q_{p_{+}^{3}}^{2}\right)\right) \cos \psi_{-} \\
& +\left(-2 q_{p_{-}^{0}} q_{p_{-}^{1}}\left(q_{p_{+}^{0}} q_{p_{+}^{2}}+q_{p_{+}^{1}} q_{p_{+}^{3}}\right)-2 q_{p_{-}^{2}} q_{p_{-}^{3}}\left(q_{p_{+}^{0}} q_{p_{+}^{2}}\right.\right. \\
& \left.+q_{p_{+}^{1}} q_{p_{+}^{3}}\right)+q_{p_{-}^{0}} q_{p_{-}^{3}}\left(-q_{p_{+}^{0}}^{2}+q_{p_{+}^{1}}^{2}+q_{p_{+}^{2}}^{2}-q_{p_{+}^{3}}^{2}\right) \\
& \left.+q_{p_{-}^{1}} q_{p_{-}^{2}}\left(q_{p_{+}^{0}}^{2}-q_{p_{+}^{1}}^{2}-q_{p_{+}^{2}}^{2}+q_{p_{+}^{3}}^{2}\right)\right) \sin \psi_{-},-2\left(q _ { p _ { - } ^ { 3 } } \left(q_{p_{-}^{1}} q_{p_{+}^{0}} q_{p_{+}^{1}}\right.\right. \\
& \left.+q_{p_{-}^{2}} q_{p_{+}^{0}} q_{p_{+}^{2}}+q_{p_{-}^{2}} q_{p_{+}^{1}} q_{p_{+}^{3}}-q_{p_{-}^{1}} q_{p_{+}^{2}} q_{p_{+}^{3}}\right)-q_{p_{-}^{0}}\left(-q_{p_{-}^{2}} q_{p_{+}^{0}} q_{p_{+}^{1}}\right. \\
& \left.\left.+q_{p_{-}^{1}} q_{p_{+}^{0}} q_{p_{+}^{2}}+q_{p_{-}^{1}} q_{p_{+}^{1}} q_{p_{+}^{3}}+q_{p_{-}^{2}} q_{p_{+}^{2}} q_{p_{+}^{3}}\right)\right) \cos \psi_{-} \\
& +\left(q_{p_{-}^{0}}^{2}\left(q_{p_{+}^{0}} q_{p_{+}^{2}}+q_{p_{+}^{1}} q_{p_{+}^{3}}\right)-q_{p_{-}^{1}}^{2}\left(q_{p_{+}^{0}} q_{p_{+}^{2}}+q_{p_{+}^{1}} q_{p_{+}^{3}}\right)\right. \\
& +\left(q_{p_{-}^{2}}-q_{p_{-}^{3}}\right)\left(q_{p_{-}^{2}}+q_{p_{-}^{3}}\right)\left(q_{p_{+}^{0}} q_{p_{+}^{2}}+q_{p_{+}^{1}} q_{p_{+}^{3}}\right)+2 q_{p_{-}^{1}} q_{p_{-}^{2}}\left(q_{p_{+}^{0}} q_{p_{+}^{1}}\right. \\
& \left.\left.-q_{p_{+}^{2}} q_{p_{+}^{3}}\right)+2 q_{p_{-}^{0}} q_{p_{-}^{3}}\left(-q_{p_{+}^{0}} q_{p_{+}^{1}}+q_{p_{+}^{2}} q_{p_{+}^{3}}\right)\right) \sin \psi_{-}, \\
& a_{-}\left(q_{p_{-}^{0}}^{2}+q_{p_{-}^{1}}^{2}-q_{p_{-}^{2}}^{2}-q_{p_{-}^{3}}^{2}\right)-2\left(d_{+} q_{p_{+}^{0}} q_{p_{+}^{2}}+d_{+} q_{p_{+}^{1}} q_{p_{+}^{3}}-q_{p_{-}^{1}} q_{d_{-}^{0}}\right. \\
& +q_{p_{-}^{0}} q_{d_{-}^{1}}-q_{p_{-}^{3}} q_{d_{-}^{2}}+q_{p_{-}^{2}} q_{d_{-}^{3}}+q_{p_{+}^{1}} q_{d_{+}^{0}}-q_{p_{+}^{0}} q_{d_{+}^{1}}+q_{p_{+}^{3}} q_{d_{+}^{2}}
\end{aligned}
$$




$$
\begin{aligned}
& \left.-q_{p_{+}^{2}} q_{d_{+}^{3}}\right), a_{-} q_{p_{-}^{1}} q_{p_{-}^{2}}+a_{-} q_{p_{-}^{0}} q_{p_{-}^{3}}+d_{+} q_{p_{+}^{0}} q_{p_{+}^{1}} \\
& -d_{+} q_{p_{+}^{2}} q_{p_{+}^{3}}+q_{p_{-}^{2}} q_{d_{-}^{0}}-q_{p_{-}^{3}} q_{d_{-}^{1}}-q_{p_{-}^{0}} q_{d_{-}^{2}}+q_{p_{-}^{1}} q_{d_{-}^{3}}-q_{p_{+}^{2}} q_{d_{+}^{0}} \\
& +q_{p_{+}^{3}} q_{d_{+}^{1}}+q_{p_{+}^{0}} q_{d_{+}^{2}}-q_{p_{+}^{1}} q_{d_{+}^{3}}, a_{-}\left(-2 q_{p_{-}^{0}} q_{p_{-}^{2}}+2 q_{p_{-}^{1}} q_{p_{-}^{3}}\right) \\
& +d_{+}\left(-q_{p_{+}^{0}}^{2}+q_{p_{+}^{1}}^{2}+q_{p_{+}^{2}}^{2}-q_{p_{+}^{3}}^{2}\right)+2\left(q_{p_{-}^{3}} q_{d_{-}^{0}}+q_{p_{-}^{2}} q_{d_{-}^{1}}\right. \\
& \left.-q_{p_{-}^{1}} q_{d_{-}^{2}}^{2}-q_{p_{-}^{0}} q_{d_{-}^{3}}^{3}-q_{p_{+}^{3}} q_{d_{+}^{0}}-q_{p_{+}^{2}} q_{d_{+}^{1}}+q_{p_{+}^{1}} q_{d_{+}^{2}}+q_{p_{+}^{0}} q_{d_{+}^{3}}\right), \\
& q_{p_{-}^{0}} q_{d_{-}^{0}}+q_{p_{-}^{1}} q_{d_{-}^{1}}+q_{p_{-}^{2}} q_{d_{-}^{2}}+q_{p_{-}^{3}} q_{d_{-}^{3}}, q_{p_{+}^{0}} q_{d_{+}^{0}}+q_{p_{+}^{1}} q_{d_{+}^{1}} \\
& +q_{p_{+}^{2}} q_{d_{+}^{2}}+q_{p_{+}^{3}} q_{d_{+}^{3}}, q_{p_{-}^{0}}^{2}+q_{p_{-}^{1}}^{2}+q_{p_{-}^{2}}^{2}+q_{p_{-}^{3}}^{2}-1, \\
& \left.q_{p_{+}^{0}}^{2}+q_{p_{+}^{1}}^{2}+q_{p_{+}^{2}}^{2}+q_{p_{+}^{3}}^{2}-1\right]^{T}
\end{aligned}
$$

2. P-joint:

$$
\begin{aligned}
& \left(q_{p^{i}} ; q_{d^{i}}\right)_{ \pm}^{i=0,1,2,3} \mapsto\left[\left(q_{p_{-}^{0}}^{2}\left(q_{p_{+}^{0}} q_{p_{+}^{2}}+q_{p_{+}^{1}} q_{p_{+}^{3}}\right)-q_{p_{-}^{1}}^{2}\left(q_{p_{+}^{0}} q_{p_{+}^{2}}+q_{p_{+}^{1}} q_{p_{+}^{3}}\right)\right.\right. \\
& -\left(q_{p_{-}^{2}}-q_{p_{-}^{3}}\right)\left(q_{p_{-}^{2}}+q_{p_{-}^{3}}\right)\left(q_{p_{+}^{0}} q_{p_{+}^{2}}+q_{p_{+}^{1}} q_{p_{+}^{3}}\right)+q_{p_{-}^{0}} q_{p_{-}^{2}}\left(-q_{p_{+}^{0}}^{2}\right. \\
& \left.\left.+q_{p_{+}^{1}}^{2}+q_{p_{+}^{2}}^{2}-q_{p_{+}^{3}}^{2}\right)+q_{p_{-}^{1}} q_{p_{-}^{3}}\left(-q_{p_{+}^{0}}^{2}+q_{p_{+}^{1}}^{2}+q_{p_{+}^{2}}^{2}-q_{p_{+}^{3}}^{2}\right)\right) \cos \psi_{-} \\
& +\left(-2 q_{p_{-}^{0}} q_{p_{-}^{1}}\left(q_{p_{+}^{0}} q_{p_{+}^{2}}+q_{p_{+}^{1}} q_{p_{+}^{3}}\right)-2 q_{p_{-}^{2}} q_{p_{-}^{3}}\left(q_{p_{+}^{0}} q_{p_{+}^{2}}\right.\right. \\
& \left.+q_{p_{+}^{1}} q_{p_{+}^{3}}\right)+q_{p_{-}^{0}} q_{p_{-}^{3}}\left(-q_{p_{+}^{0}}^{2}+q_{p_{+}^{1}}^{2}+q_{p_{+}^{2}}^{2}-q_{p_{+}^{3}}^{2}\right) \\
& \left.+q_{p_{-}^{1}} q_{p_{-}^{2}}\left(q_{p_{+}^{0}}^{2}-q_{p_{+}^{1}}^{2}-q_{p_{+}^{2}}^{2}+q_{p_{+}^{3}}^{2}\right)\right) \sin \psi_{-},-2\left(q _ { p _ { - } ^ { 3 } } \left(q_{p_{-}^{1}} q_{p_{+}^{0}} q_{p_{+}^{1}}\right.\right. \\
& \left.+q_{p_{-}^{2}} q_{p_{+}^{0}} q_{p_{+}^{2}}+q_{p_{-}^{2}} q_{p_{+}^{1}} q_{p_{+}^{3}}-q_{p_{-}^{1}} q_{p_{+}^{2}} q_{p_{+}^{3}}\right)-q_{p_{-}^{0}}\left(-q_{p_{-}^{2}} q_{p_{+}^{0}} q_{p_{+}^{1}}\right. \\
& \left.\left.+q_{p_{-}^{1}} q_{p_{+}^{0}} q_{p_{+}^{2}}+q_{p_{-}^{1}} q_{p_{+}^{1}} q_{p_{+}^{3}}+q_{p_{-}^{2}} q_{p_{+}^{2}} q_{p_{+}^{3}}\right)\right) \cos \psi_{-} \\
& +\left(q_{p_{-}^{0}}^{2}\left(q_{p_{+}^{0}} q_{p_{+}^{2}}+q_{p_{+}^{1}} q_{p_{+}^{3}}\right)-q_{p_{-}^{1}}^{2}\left(q_{p_{+}^{0}} q_{p_{+}^{2}}+q_{p_{+}^{1}} q_{p_{+}^{3}}\right)\right. \\
& +\left(q_{p_{-}^{2}}-q_{p_{-}^{3}}\right)\left(q_{p_{-}^{2}}+q_{p_{-}^{3}}\right)\left(q_{p_{+}^{0}} q_{p_{+}^{2}}+q_{p_{+}^{1}} q_{p_{+}^{3}}\right)+2 q_{p_{-}^{1}} q_{p_{-}^{2}}\left(q_{p_{+}^{0}} q_{p_{+}^{1}}\right. \\
& \left.\left.-q_{p_{+}^{2}} q_{p_{+}^{3}}\right)+2 q_{p_{-}^{0}} q_{p_{-}^{3}}\left(-q_{p_{+}^{0}} q_{p_{+}^{1}}+q_{p_{+}^{2}} q_{p_{+}^{3}}\right)\right) \sin \psi_{-}, \\
& -2\left(-q_{p_{-}^{3}}\left(q_{p_{-}^{0}} q_{p_{+}^{0}} q_{p_{+}^{1}}+q_{p_{-}^{2}} q_{p_{+}^{1}} q_{p_{+}^{2}}-q_{p_{-}^{2}} q_{p_{+}^{0}} q_{p_{+}^{3}}+q_{p_{-}^{0}} q_{p_{+}^{2}} q_{p_{+}^{3}}\right)\right. \\
& +q_{p_{-}^{1}}\left(q_{p_{-}^{2}} q_{p_{+}^{0}} q_{p_{+}^{1}}-q_{p_{-}^{0}} q_{p_{+}^{1}} q_{p_{+}^{2}}+q_{p_{-}^{0}} q_{p_{+}^{0}} q_{p_{+}^{3}}\right. \\
& \left.\left.+q_{p_{-}^{2}} q_{p_{+}^{2}} q_{p_{+}^{3}}\right)\right) \cos \psi_{-}-\left(2\left(q_{p_{-}^{0}} q_{p_{-}^{2}}+q_{p_{-}^{1}} q_{p_{-}^{3}}\right) q_{p_{+}^{0}} q_{p_{+}^{1}}\right. \\
& +\left(-q_{p_{-}^{0}}^{2}+q_{p_{-}^{1}}^{2}+q_{p_{-}^{2}}^{2}-q_{p_{-}^{3}}^{2}\right) q_{p_{+}^{1}} q_{p_{+}^{2}}+\left(q_{p_{-}^{0}}^{2}\right. \\
& \left.-q_{p_{-}^{1}}^{2}-q_{p_{-}^{2}}^{2}+q_{p_{-}^{3}}^{2}\right) q_{p_{+}^{0}} q_{p_{+}^{3}} \\
& \left.+2\left(q_{p_{-}^{0}} q_{p_{-}^{2}}+q_{p_{-}^{1}} q_{p_{-}^{3}}\right) q_{p_{+}^{2}} q_{p_{+}^{3}}\right) \sin \psi_{-},\left(q_{p_{-}^{0}}^{2}\left(q_{p_{+}^{1}} q_{p_{+}^{2}}-q_{p_{+}^{0}} q_{p_{+}^{3}}\right)\right. \\
& +\left(q_{p_{-}^{2}}-q_{p_{-}^{3}}\right)\left(q_{p_{-}^{2}}+q_{p_{-}^{3}}\right)\left(q_{p_{+}^{1}} q_{p_{+}^{2}}-q_{p_{+}^{0}} q_{p_{+}^{3}}\right)
\end{aligned}
$$


195

$$
\begin{aligned}
& +q_{p_{-}^{1}}^{2}\left(-q_{p_{+}^{1}} q_{p_{+}^{2}}+q_{p_{+}^{0}} q_{p_{+}^{3}}\right)+q_{p_{-}^{0}} q_{p_{-}^{3}}\left(q_{p_{+}^{0}}^{2}-q_{p_{+}^{1}}^{2}+q_{p_{+}^{2}}^{2}\right. \\
& \left.\left.-q_{p_{+}^{3}}^{2}\right)+q_{p_{-}^{1}} q_{p_{-}^{2}}\left(-q_{p_{+}^{0}}^{2}+q_{p_{+}^{1}}^{2}-q_{p_{+}^{2}}^{2}+q_{p_{+}^{3}}^{2}\right)\right) \cos \psi_{-} \\
& +\left(-2 q_{p_{-}^{0}} q_{p_{-}^{1}} q_{p_{+}^{1}} q_{p_{+}^{2}}+2 q_{p_{-}^{0}} q_{p_{-}^{1}} q_{p_{+}^{0}} q_{p_{+}^{3}}+2 q_{p_{-}^{2}} q_{p_{-}^{3}}\left(q_{p_{+}^{1}} q_{p_{+}^{2}}\right.\right. \\
& \left.-q_{p_{+}^{0}} q_{p_{+}^{3}}\right)+q_{p_{-}^{0}} q_{p_{-}^{2}}\left(-q_{p_{+}^{0}}^{2}+q_{p_{+}^{1}}^{2}-q_{p_{+}^{2}}^{2}+q_{p_{+}^{3}}^{2}\right) \\
& \left.+q_{p_{-}^{1}} q_{p_{-}^{3}}\left(-q_{p_{+}^{0}}^{2}+q_{p_{+}^{1}}^{2}-q_{p_{+}^{2}}^{2}+q_{p_{+}^{3}}^{2}\right)\right) \sin \psi_{-}, a_{-}\left(\left(q_{p_{-}^{2}}^{2}\right.\right. \\
& \left.+q_{p_{-}^{3}}^{2}\right)\left(q_{p_{+}^{1}} q_{p_{+}^{2}}-q_{p_{+}^{0}} q_{p_{+}^{3}}\right)+q_{p_{-}^{0}}^{2}\left(-q_{p_{+}^{1}} q_{p_{+}^{2}}+q_{p_{+}^{0}} q_{p_{+}^{3}}\right) \\
& +q_{p_{-}^{1}}^{2}\left(-q_{p_{+}^{1}} q_{p_{+}^{2}}+q_{p_{+}^{0}} q_{p_{+}^{3}}\right)+2 q_{p_{-}^{0}} q_{p_{-}^{2}}\left(q_{p_{+}^{0}} q_{p_{+}^{1}}\right. \\
& \left.+q_{p_{+}^{2}} q_{p_{+}^{3}}\right)+q_{p_{-}^{0}} q_{p_{-}^{3}}\left(-q_{p_{+}^{0}}^{2}+q_{p_{+}^{1}}^{2}-q_{p_{+}^{2}}^{2}+q_{p_{+}^{3}}^{2}\right) \\
& -q_{p_{-}^{1}}\left(2 q_{p_{-}^{3}}\left(q_{p_{+}^{0}} q_{p_{+}^{1}}+q_{p_{+}^{2}} q_{p_{+}^{3}}\right)\right. \\
& \left.\left.+q_{p_{-}^{2}}\left(q_{p_{+}^{0}}^{2}-q_{p_{+}^{1}}^{2}+q_{p_{+}^{2}}^{2}-q_{p_{+}^{3}}^{2}\right)\right)\right)-2 q_{p_{-}^{3}} q_{p_{+}^{0}} q_{p_{+}^{1}} q_{d_{-}^{0}} \\
& -2 q_{p_{-}^{1}} q_{p_{+}^{1}} q_{p_{+}^{2}} q_{d_{-}^{0}}+2 q_{p_{-}^{1}} q_{p_{+}^{0}} q_{p_{+}^{3}} q_{d_{-}^{0}}-2 q_{p_{-}^{3}} q_{p_{+}^{2}} q_{p_{+}^{3}} q_{d_{-}^{0}} \\
& +q_{p_{-}^{3}} q_{p_{+}^{0}}^{2} q_{d_{-}^{1}}-q_{p_{-}^{3}} q_{p_{+}^{1}}^{2} q_{d_{-}^{1}}+2 q_{p_{-}^{0}} q_{p_{+}^{1}} q_{p_{+}^{2}} q_{d_{-}^{1}}+q_{p_{-}^{3}} q_{p_{+}^{2}}^{2} q_{d_{-}^{1}} \\
& -2 q_{p_{-}^{0}} q_{p_{+}^{0}} q_{p_{+}^{3}} q_{d_{-}^{1}}-q_{p_{-}^{3}} q_{p_{+}^{3}}^{2} q_{d_{-}^{1}}+q_{p_{-}^{0}} q_{p_{+}^{0}}^{2} q_{d_{-}^{2}}+2 q_{p_{-}^{1}} q_{p_{+}^{0}} q_{p_{+}^{1}} q_{d_{-}^{2}} \\
& -q_{p_{-}^{0}} q_{p_{+}^{1}}^{2} q_{d_{-}^{2}}-2 q_{p_{-}^{3}} q_{p_{+}^{1}} q_{p_{+}^{2}} q_{d_{-}^{2}}+q_{p_{-}^{0}} q_{p_{+}^{2}}^{2} q_{d_{-}^{2}}+2 q_{p_{-}^{3}} q_{p_{+}^{0}} q_{p_{+}^{3}} q_{d_{-}^{2}} \\
& +2 q_{p_{-}^{1}} q_{p_{+}^{2}} q_{p_{+}^{3}} q_{d_{-}^{2}}-q_{p_{-}^{0}} q_{p_{+}^{3}}^{2} q_{d_{-}^{2}}-q_{p_{-}^{1}} q_{p_{+}^{0}}^{2} q_{d_{-}^{3}}+2 q_{p_{-}^{0}} q_{p_{+}^{0}} q_{p_{+}^{1}} q_{d_{-}^{3}} \\
& +q_{p_{-}^{1}} q_{p_{+}^{1}}^{2} q_{d_{-}^{3}}-q_{p_{-}^{1}} q_{p_{+}^{2}}^{2} q_{d_{-}^{3}}+2 q_{p_{-}^{0}} q_{p_{+}^{2}} q_{p_{+}^{3}} q_{d_{-}^{3}} \\
& +q_{p_{-}^{1}} q_{p_{+}^{3}}^{2} q_{d_{-}^{3}}-q_{p_{-}^{2}}\left(q_{p_{+}^{0}}^{2} q_{d_{-}^{0}}-q_{p_{+}^{1}}^{2} q_{d_{-}^{0}}+q_{p_{+}^{2}}^{2} q_{d_{-}^{0}}\right. \\
& -q_{p_{+}^{3}}^{2} q_{d_{-}^{0}}+2 q_{p_{+}^{2}} q_{p_{+}^{3}} q_{d_{-}^{1}}-2 q_{p_{+}^{1}} q_{p_{+}^{2}} q_{d_{-}^{3}}+2 q_{p_{+}^{0}}\left(q_{p_{+}^{1}} q_{d_{-}^{1}}\right. \\
& \left.\left.+q_{p_{+}^{3}} q_{d_{-}^{3}}\right)\right)+q_{p_{+}^{0}}^{2} q_{p_{+}^{2}} q_{d_{+}^{0}}+q_{p_{+}^{1}}^{2} q_{p_{+}^{2}} q_{d_{+}^{0}}+q_{p_{+}^{2}}^{3} q_{d_{+}^{0}} \\
& +q_{p_{+}^{2}} q_{p_{+}^{3}}^{2} q_{d_{+}^{0}}+q_{p_{+}^{0}}^{2} q_{p_{+}^{3}} q_{d_{+}^{1}}+q_{p_{+}^{1}}^{2} q_{p_{+}^{3}} q_{d_{+}^{1}}+q_{p_{+}^{2}}^{2} q_{p_{+}^{3}} q_{d_{+}^{1}} \\
& +q_{p_{+}^{3}}^{3} q_{d_{+}^{1}}-q_{p_{+}^{0}}^{3} q_{d_{+}^{2}}-q_{p_{+}^{0}} q_{p_{+}^{1}}^{2} q_{d_{+}^{2}}-q_{p_{+}^{0}} q_{p_{+}^{2}}^{2} q_{d_{+}^{2}} \\
& -q_{p_{+}^{0}} q_{p_{+}^{3}}^{2} q_{d_{+}^{2}}-q_{p_{+}^{0}}^{2} q_{p_{+}^{1}} q_{d_{+}^{3}}-q_{p_{+}^{1}}^{3} q_{d_{+}^{3}}-q_{p_{+}^{1}} q_{p_{+}^{2}}^{2} q_{d_{+}^{3}} \\
& -q_{p_{+}^{1}} q_{p_{+}^{3}}^{2} q_{d_{+}^{3}}, q_{p_{-}^{0}} q_{d_{-}^{0}}+q_{p_{-}^{1}} q_{d_{-}^{1}}+q_{p_{-}^{2}} q_{d_{-}^{2}}+q_{p_{-}^{3}} q_{d_{-}^{3}}, \\
& q_{p_{+}^{0}} q_{d_{+}^{0}}+q_{p_{+}^{1}} q_{d_{+}^{1}}+q_{p_{+}^{2}} q_{d_{+}^{2}}+q_{p_{+}^{3}} q_{d_{+}^{3}}, q_{p_{-}^{0}}^{2}+q_{p_{-}^{1}}^{2}+q_{p_{-}^{2}}^{2}+q_{p_{-}^{3}}^{2} \\
& \left.-1, q_{p_{+}^{0}}^{2}+q_{p_{+}^{1}}^{2}+q_{p_{+}^{2}}^{2}+q_{p_{+}^{3}}^{2}-1\right]^{T}
\end{aligned}
$$

3. C-joint:

$$
\left(q_{p^{i}} ; q_{d^{i}}\right)_{ \pm}^{i=0,1,2,3} \mapsto\left[\left(q_{p_{-}^{0}}^{2}\left(q_{p_{+}^{0}} q_{p_{+}^{2}}+q_{p_{+}^{1}} q_{p_{+}^{3}}\right)-q_{p_{-}^{1}}^{2}\left(q_{p_{+}^{0}} q_{p_{+}^{2}}+q_{p_{+}^{1}} q_{p_{+}^{3}}\right)\right.\right.
$$




$$
\begin{aligned}
& -\left(q_{p_{-}^{2}}-q_{p_{-}^{3}}\right)\left(q_{p_{-}^{2}}+q_{p_{-}^{3}}\right)\left(q_{p_{+}^{0}} q_{p_{+}^{2}}+q_{p_{+}^{1}} q_{p_{+}^{3}}\right)+q_{p_{-}^{0}} q_{p_{-}^{2}}\left(-q_{p_{+}^{0}}^{2}\right. \\
& \left.\left.+q_{p_{+}^{1}}^{2}+q_{p_{+}^{2}}^{2}-q_{p_{+}^{3}}^{2}\right)+q_{p_{-}^{1}} q_{p_{-}^{3}}\left(-q_{p_{+}^{0}}^{2}+q_{p_{+}^{1}}^{2}+q_{p_{+}^{2}}^{2}-q_{p_{+}^{3}}^{2}\right)\right) \cos \psi_{-} \\
& +\left(-2 q_{p_{-}^{0}} q_{p_{-}^{1}}\left(q_{p_{+}^{0}} q_{p_{+}^{2}}+q_{p_{+}^{1}} q_{p_{+}^{3}}\right)-2 q_{p_{-}^{2}} q_{p_{-}^{3}}\left(q_{p_{+}^{0}} q_{p_{+}^{2}}\right.\right. \\
& \left.+q_{p_{+}^{1}} q_{p_{+}^{3}}\right)+q_{p_{-}^{0}} q_{p_{-}^{3}}\left(-q_{p_{+}^{0}}^{2}+q_{p_{+}^{1}}^{2}+q_{p_{+}^{2}}^{2}-q_{p_{+}^{3}}^{2}\right) \\
& \left.+q_{p_{-}^{1}} q_{p_{-}^{2}}\left(q_{p_{+}^{0}}^{2}-q_{p_{+}^{1}}^{2}-q_{p_{+}^{2}}^{2}+q_{p_{+}^{3}}^{2}\right)\right) \sin \psi_{-},-2\left(q _ { p _ { - } ^ { 3 } } \left(q_{p_{-}^{1}} q_{p_{+}^{0}} q_{p_{+}^{1}}\right.\right. \\
& \left.+q_{p_{-}^{2}} q_{p_{+}^{0}} q_{p_{+}^{2}}+q_{p_{-}^{2}} q_{p_{+}^{1}} q_{p_{+}^{3}}-q_{p_{-}^{1}} q_{p_{+}^{2}} q_{p_{+}^{3}}\right)-q_{p_{-}^{0}}\left(-q_{p_{-}^{2}} q_{p_{+}^{0}} q_{p_{+}^{1}}\right. \\
& \left.\left.+q_{p_{-}^{1}} q_{p_{+}^{0}} q_{p_{+}^{2}}+q_{p_{-}^{1}} q_{p_{+}^{1}} q_{p_{+}^{3}}+q_{p_{-}^{2}} q_{p_{+}^{2}} q_{p_{+}^{3}}\right)\right) \cos \psi_{-} \\
& +\left(q_{p_{-}^{0}}^{2}\left(q_{p_{+}^{0}} q_{p_{+}^{2}}+q_{p_{+}^{1}} q_{p_{+}^{3}}\right)-q_{p_{-}^{1}}^{2}\left(q_{p_{+}^{0}} q_{p_{+}^{2}}+q_{p_{+}^{1}} q_{p_{+}^{3}}\right)\right. \\
& +\left(q_{p_{-}^{2}}-q_{p_{-}^{3}}\right)\left(q_{p_{-}^{2}}+q_{p_{-}^{3}}\right)\left(q_{p_{+}^{0}} q_{p_{+}^{2}}+q_{p_{+}^{1}} q_{p_{+}^{3}}\right) \\
& +2 q_{p_{-}^{1}} q_{p_{-}^{2}}\left(q_{p_{+}^{0}} q_{p_{+}^{1}}-q_{p_{+}^{2}} q_{p_{+}^{3}}\right)+2 q_{p_{-}^{0}} q_{p_{-}^{3}}\left(-q_{p_{+}^{0}} q_{p_{+}^{1}}\right. \\
& \left.\left.+q_{p_{+}^{2}} q_{p_{+}^{3}}\right)\right) \sin \psi_{-}, a_{-}\left(4 q_{p_{-}^{0}} q_{p_{-}^{2}}\left(q_{p_{+}^{0}} q_{p_{+}^{2}}+q_{p_{+}^{1}} q_{p_{+}^{3}}\right)\right. \\
& -4 q_{p_{-}^{1}} q_{p_{-}^{3}}\left(q_{p_{+}^{0}} q_{p_{+}^{2}}+q_{p_{+}^{1}} q_{p_{+}^{3}}\right)+q_{p_{-}^{0}}^{2}\left(q_{p_{+}^{0}}^{2}-q_{p_{+}^{1}}^{2}-q_{p_{+}^{2}}^{2}+q_{p_{+}^{3}}^{2}\right) \\
& +q_{p_{-}^{1}}^{2}\left(q_{p_{+}^{0}}^{2}-q_{p_{+}^{1}}^{2}-q_{p_{+}^{2}}^{2}+q_{p_{+}^{3}}^{2}\right)-\left(q_{p_{-}^{2}}^{2}+q_{p_{-}^{3}}^{2}\right)\left(q_{p_{+}^{0}}^{2}-q_{p_{+}^{1}}^{2}-q_{p_{+}^{2}}^{2}\right. \\
& \left.\left.+q_{p_{+}^{3}}^{2}\right)\right)-2\left(2 q_{p_{-}^{3}}\left(q_{p_{+}^{0}} q_{p_{+}^{2}}+q_{p_{+}^{1}} q_{p_{+}^{3}}\right) q_{d_{-}^{0}}+q_{p_{-}^{1}}\left(-q_{p_{+}^{0}}^{2}\right.\right. \\
& \left.+q_{p_{+}^{1}}^{2}+q_{p_{+}^{2}}^{2}-q_{p_{+}^{3}}^{2}\right) q_{d_{-}^{0}}+q_{p_{-}^{0}} q_{p_{+}^{0}}^{2} q_{d_{-}^{1}}-q_{p_{-}^{0}} q_{p_{+}^{1}}^{2} q_{d_{-}^{1}} \\
& +2 q_{p_{-}^{2}} q_{p_{+}^{0}} q_{p_{+}^{2}} q_{d_{-}^{1}}-q_{p_{-}^{0}} q_{p_{+}^{2}}^{2} q_{d_{-}^{1}}+2 q_{p_{-}^{2}} q_{p_{+}^{1}} q_{p_{+}^{3}} q_{d_{-}^{1}}+q_{p_{-}^{0}} q_{p_{+}^{3}}^{2} q_{d_{-}^{1}} \\
& -2 q_{p_{-}^{1}}\left(q_{p_{+}^{0}} q_{p_{+}^{2}}+q_{p_{+}^{1}} q_{p_{+}^{3}}\right) q_{d_{-}^{2}}+q_{p_{-}^{3}}\left(-q_{p_{+}^{0}}^{2}+q_{p_{+}^{1}}^{2}+q_{p_{+}^{2}}^{2}\right. \\
& \left.-q_{p_{+}^{3}}^{2}\right) q_{d_{-}^{2}}+q_{p_{-}^{2}} q_{p_{+}^{0}}^{2} q_{d_{-}^{3}}-q_{p_{-}^{2}} q_{p_{+}^{1}}^{2} q_{d_{-}^{3}}-2 q_{p_{-}^{0}} q_{p_{+}^{0}} q_{p_{+}^{2}} q_{d_{-}^{3}} \\
& -q_{p_{-}^{2}} q_{p_{+}^{2}}^{2} q_{d_{-}^{3}}-2 q_{p_{-}^{0}} q_{p_{+}^{1}} q_{p_{+}^{3}} q_{d_{-}^{3}}+q_{p_{-}^{2}} q_{p_{+}^{3}}^{2} q_{d_{-}^{3}}+q_{p_{+}^{0}}^{2} q_{p_{+}^{1}} q_{d_{+}^{0}} \\
& -q_{p_{+}^{1}}^{3} q_{d_{+}^{0}}-q_{p_{+}^{1}} q_{p_{+}^{2}}^{2} q_{d_{+}^{0}}-2 q_{p_{+}^{0}} q_{p_{+}^{2}} q_{p_{+}^{3}} q_{d_{+}^{0}}-q_{p_{+}^{1}} q_{p_{+}^{3}}^{2} q_{d_{+}^{0}}-q_{p_{+}^{0}}^{3} q_{d_{+}^{1}} \\
& +q_{p_{+}^{0}} q_{p_{+}^{1}}^{2} q_{d_{+}^{1}}-q_{p_{+}^{0}} q_{p_{+}^{2}}^{2} q_{d_{+}^{1}}-2 q_{p_{+}^{1}} q_{p_{+}^{2}} q_{p_{+}^{3}} q_{d_{+}^{1}}-q_{p_{+}^{0}} q_{p_{+}^{3}}^{2} q_{d_{+}^{1}} \\
& +2 q_{p_{+}^{0}} q_{p_{+}^{1}} q_{p_{+}^{2}} q_{d_{+}^{2}}+q_{p_{+}^{0}}^{2} q_{p_{+}^{3}} q_{d_{+}^{2}}+q_{p_{+}^{1}}^{2} q_{p_{+}^{3}} q_{d_{+}^{2}} \\
& -q_{p_{+}^{2}}^{2} q_{p_{+}^{3}} q_{d_{+}^{2}}+q_{p_{+}^{3}}^{3} q_{d_{+}^{2}}+\left(q_{p_{+}^{2}}\left(q_{p_{+}^{0}}^{2}+q_{p_{+}^{1}}^{2}+q_{p_{+}^{2}}^{2}\right)+2 q_{p_{+}^{0}} q_{p_{+}^{1}} q_{p_{+}^{3}}\right. \\
& \left.\left.-q_{p_{+}^{2}} q_{p_{+}^{3}}^{2}\right) q_{d_{+}^{3}}\right), a_{-}\left(2 q_{p_{-}^{1}} q_{p_{-}^{2}}\left(q_{p_{+}^{0}} q_{p_{+}^{2}}+q_{p_{+}^{1}} q_{p_{+}^{3}}\right)\right. \\
& +2 q_{p_{-}^{0}} q_{p_{-}^{3}}\left(q_{p_{+}^{0}} q_{p_{+}^{2}}+q_{p_{+}^{1}} q_{p_{+}^{3}}\right)+q_{p_{-}^{0}}^{2}\left(q_{p_{+}^{0}} q_{p_{+}^{1}}-q_{p_{+}^{2}} q_{p_{+}^{3}}\right) \\
& +q_{p_{-}^{1}}^{2}\left(q_{p_{+}^{0}} q_{p_{+}^{1}}-q_{p_{+}^{2}} q_{p_{+}^{3}}\right)-\left(q_{p_{-}^{2}}^{2}+q_{p_{-}^{3}}^{2}\right)\left(q_{p_{+}^{0}} q_{p_{+}^{1}}\right. \\
& \left.\left.-q_{p_{+}^{2}} q_{p_{+}^{3}}\right)\right)-2\left(q_{p_{-}^{0}} q_{p_{+}^{0}} q_{p_{+}^{1}} q_{d_{-}^{1}}+q_{p_{-}^{3}} q_{p_{+}^{0}} q_{p_{+}^{2}} q_{d_{-}^{1}}+q_{p_{-}^{3}} q_{p_{+}^{1}} q_{p_{+}^{3}} q_{d_{-}^{1}}\right. \\
& -q_{p_{-}^{0}} q_{p_{+}^{2}} q_{p_{+}^{3}} q_{d_{-}^{1}}-q_{p_{-}^{3}} q_{p_{+}^{0}} q_{p_{+}^{1}} q_{d_{-}^{2}}+q_{p_{-}^{0}} q_{p_{+}^{0}} q_{p_{+}^{2}} q_{d_{-}^{2}}
\end{aligned}
$$




$$
\begin{aligned}
& +q_{p_{-}^{0}} q_{p_{+}^{1}} q_{p_{+}^{3}} q_{d_{-}^{2}}+q_{p_{-}^{3}} q_{p_{+}^{2}} q_{p_{+}^{3}} q_{d_{-}^{2}}-q_{p_{-}^{1}}\left(q_{p_{+}^{0}} q_{p_{+}^{1}} q_{d_{-}^{0}}\right. \\
& \left.-q_{p_{+}^{2}} q_{p_{+}^{3}} q_{d_{-}^{0}}+q_{p_{+}^{0}} q_{p_{+}^{2}} q_{d_{-}^{3}}+q_{p_{+}^{1}} q_{p_{+}^{3}} q_{d_{-}^{3}}\right)-q_{p_{-}^{2}}\left(q_{p_{+}^{0}} q_{p_{+}^{2}} q_{d_{-}^{0}}\right. \\
& \left.+q_{p_{+}^{1}} q_{p_{+}^{3}} q_{d_{-}^{0}}-q_{p_{+}^{0}} q_{p_{+}^{1}} q_{d_{-}^{3}}+q_{p_{+}^{2}} q_{p_{+}^{3}} q_{d_{-}^{3}}\right)+q_{p_{+}^{0}} q_{p_{+}^{1}}^{2} q_{d_{+}^{0}} \\
& +q_{p_{+}^{0}} q_{p_{+}^{2}}^{2} q_{d_{+}^{0}}-q_{p_{+}^{0}}^{2} q_{p_{+}^{1}} q_{d_{+}^{1}}-q_{p_{+}^{1}} q_{p_{+}^{3}}^{2} q_{d_{+}^{1}}-q_{p_{+}^{0}}^{2} q_{p_{+}^{2}} q_{d_{+}^{2}}^{2} \\
& \left.-q_{p_{+}^{2}}^{2} q_{p_{+}^{3}}^{2} q_{d_{+}^{2}}+\left(q_{p_{+}^{1}}^{2}+q_{p_{+}^{2}}^{2}\right) q_{p_{+}^{3}}^{3} q_{d_{+}^{3}}\right), q_{p_{-}^{0}} q_{d_{-}^{0}}+q_{p_{-}^{1}} q_{d_{-}^{1}}+q_{p_{-}^{2}} q_{d_{-}^{2}} \\
& +q_{p_{-}^{3}} q_{d_{-}^{3}}, q_{p_{+}^{0}} q_{d_{+}^{0}}+q_{p_{+}^{1}} q_{d_{+}^{1}}+q_{p_{+}^{2}} q_{d_{+}^{2}}+q_{p_{+}^{3}} q_{d_{+}^{3}}, q_{p_{-}^{0}}^{2}+q_{p_{-}^{1}}^{2} \\
& \left.+q_{p_{-}^{2}}^{2}+q_{p_{-}^{3}}^{2}-1, q_{p_{+}^{0}}^{2}+q_{p_{+}^{1}}^{2}+q_{p_{+}^{2}}^{2}+q_{p_{+}^{3}}^{2}-1\right]^{T}
\end{aligned}
$$

4. E-joint:

$$
\begin{aligned}
& \left(q_{p^{i}} ; q_{d^{i}}\right)_{ \pm}^{i=0,1,2,3} \mapsto\left[\left(q_{p_{-}^{0}}^{2}\left(q_{p_{+}^{0}} q_{p_{+}^{2}}+q_{p_{+}^{1}} q_{p_{+}^{3}}\right)-q_{p_{-}^{1}}^{2}\left(q_{p_{+}^{0}} q_{p_{+}^{2}}+q_{p_{+}^{1}} q_{p_{+}^{3}}\right)\right.\right. \\
& -\left(q_{p_{-}^{2}}-q_{p_{-}^{3}}\right)\left(q_{p_{-}^{2}}+q_{p_{-}^{3}}\right)\left(q_{p_{+}^{0}} q_{p_{+}^{2}}+q_{p_{+}^{1}} q_{p_{+}^{3}}\right)+q_{p_{-}^{0}} q_{p_{-}^{2}}\left(-q_{p_{+}^{0}}^{2}\right. \\
& \left.\left.+q_{p_{+}^{1}}^{2}+q_{p_{+}^{2}}^{2}-q_{p_{+}^{3}}^{2}\right)+q_{p_{-}^{1}} q_{p_{-}^{3}}\left(-q_{p_{+}^{0}}^{2}+q_{p_{+}^{1}}^{2}+q_{p_{+}^{2}}^{2}-q_{p_{+}^{3}}^{2}\right)\right) \cos \psi_{-} \\
& +\left(-2 q_{p_{-}^{0}} q_{p_{-}^{1}}\left(q_{p_{+}^{0}} q_{p_{+}^{2}}+q_{p_{+}^{1}} q_{p_{+}^{3}}\right)-2 q_{p_{-}^{2}} q_{p_{-}^{3}}\left(q_{p_{+}^{0}} q_{p_{+}^{2}}\right.\right. \\
& \left.+q_{p_{+}^{1}} q_{p_{+}^{3}}\right)+q_{p_{-}^{0}} q_{p_{-}^{3}}\left(-q_{p_{+}^{0}}^{2}+q_{p_{+}^{1}}^{2}+q_{p_{+}^{2}}^{2}-q_{p_{+}^{3}}^{2}\right) \\
& \left.+q_{p_{-}^{1}} q_{p_{-}^{2}}\left(q_{p_{+}^{0}}^{2}-q_{p_{+}^{1}}^{2}-q_{p_{+}^{2}}^{2}+q_{p_{+}^{3}}^{2}\right)\right) \sin \psi_{-},-2\left(q _ { p _ { - } ^ { 3 } } \left(q_{p_{-}^{1}} q_{p_{+}^{0}} q_{p_{+}^{1}}\right.\right. \\
& \left.+q_{p_{-}^{2}} q_{p_{+}^{0}} q_{p_{+}^{2}}+q_{p_{-}^{2}} q_{p_{+}^{1}} q_{p_{+}^{3}}-q_{p_{-}^{1}} q_{p_{+}^{2}} q_{p_{+}^{3}}\right)-q_{p_{-}^{0}}\left(-q_{p_{-}^{2}} q_{p_{+}^{0}} q_{p_{+}^{1}}\right. \\
& \left.\left.+q_{p_{-}^{1}} q_{p_{+}^{0}} q_{p_{+}^{2}}+q_{p_{-}^{1}} q_{p_{+}^{1}} q_{p_{+}^{3}}+q_{p_{-}^{2}} q_{p_{+}^{2}} q_{p_{+}^{3}}\right)\right) \cos \psi_{-}+\left(q _ { p _ { - } ^ { 0 } } ^ { 2 } \left(q_{p_{+}^{0}} q_{p_{+}^{2}}\right.\right. \\
& \left.+q_{p_{+}^{1}} q_{p_{+}^{3}}\right)-q_{p_{-}^{1}}^{2}\left(q_{p_{+}^{0}} q_{p_{+}^{2}}+q_{p_{+}^{1}} q_{p_{+}^{3}}\right)+\left(q_{p_{-}^{2}}-q_{p_{-}^{3}}\right)\left(q_{p_{-}^{2}}\right. \\
& \left.+q_{p_{-}^{3}}\right)\left(q_{p_{+}^{0}} q_{p_{+}^{2}}+q_{p_{+}^{1}} q_{p_{+}^{3}}\right)+2 q_{p_{-}^{1}} q_{p_{-}^{2}}\left(q_{p_{+}^{0}} q_{p_{+}^{1}}-q_{p_{+}^{2}} q_{p_{+}^{3}}\right) \\
& \left.+2 q_{p_{-}^{0}} q_{p_{-}^{3}}\left(-q_{p_{+}^{0}} q_{p_{+}^{1}}+q_{p_{+}^{2}} q_{p_{+}^{3}}\right)\right) \sin \psi_{-}, 2 q_{p_{-}^{3}}\left(q_{p_{+}^{0}} q_{p_{+}^{2}}\right. \\
& \left.+q_{p_{+}^{1}} q_{p_{+}^{3}}\right) q_{d_{-}^{0}}+q_{p_{-}^{1}}\left(-q_{p_{+}^{0}}^{2}+q_{p_{+}^{1}}^{2}+q_{p_{+}^{2}}^{2}-q_{p_{+}^{3}}^{2}\right) q_{d_{-}^{0}} \\
& +q_{p_{-}^{0}} q_{p_{+}^{0}}^{2} q_{d_{-}^{1}}-q_{p_{-}^{0}} q_{p_{+}^{1}}^{2} q_{d_{-}^{1}}+2 q_{p_{-}^{2}} q_{p_{+}^{0}} q_{p_{+}^{2}} q_{d_{-}^{1}}-q_{p_{-}^{0}} q_{p_{+}^{2}}^{2} q_{d_{-}^{1}} \\
& +2 q_{p_{-}^{2}} q_{p_{+}^{1}} q_{p_{+}^{3}} q_{d_{-}^{1}}+q_{p_{-}^{0}} q_{p_{+}^{3}}^{2} q_{d_{-}^{1}}-2 q_{p_{-}^{1}}\left(q_{p_{+}^{0}} q_{p_{+}^{2}}\right. \\
& \left.+q_{p_{+}^{1}} q_{p_{+}^{3}}\right) q_{d_{-}^{2}}+q_{p_{-}^{3}}\left(-q_{p_{+}^{0}}^{2}+q_{p_{+}^{1}}^{2}+q_{p_{+}^{2}}^{2}-q_{p_{+}^{3}}^{2}\right) q_{d_{-}^{2}} \\
& +q_{p_{-}^{2}} q_{p_{+}^{0}}^{2} q_{d_{-}^{3}}-q_{p_{-}^{2}} q_{p_{+}^{1}}^{2} q_{d_{-}^{3}}-2 q_{p_{-}^{0}} q_{p_{+}^{0}} q_{p_{+}^{2}} q_{d_{-}^{3}}-q_{p_{-}^{2}} q_{p_{+}^{2}}^{2} q_{d_{-}^{3}} \\
& -2 q_{p_{-}^{0}} q_{p_{+}^{1}} q_{p_{+}^{3}} q_{d_{-}^{3}}+q_{p_{-}^{2}} q_{p_{+}^{3}}^{2} q_{d_{-}^{3}}+q_{p_{+}^{0}}^{2} q_{p_{+}^{1}} q_{d_{+}^{0}} \\
& -q_{p_{+}^{1}}^{3} q_{d_{+}^{0}}-q_{p_{+}^{1}} q_{p_{+}^{2}}^{2} q_{d_{+}^{0}}-2 q_{p_{+}^{0}} q_{p_{+}^{2}} q_{p_{+}^{3}} q_{d_{+}^{0}}-q_{p_{+}^{1}} q_{p_{+}^{3}}^{2} q_{d_{+}^{0}}-q_{p_{+}^{0}}^{3} q_{d_{+}^{1}} \\
& +q_{p_{+}^{0}} q_{p_{+}^{1}}^{2} q_{d_{+}^{1}}-q_{p_{+}^{0}} q_{p_{+}^{2}}^{2} q_{d_{+}^{1}}-2 q_{p_{+}^{1}} q_{p_{+}^{2}} q_{p_{+}^{3}} q_{d_{+}^{1}}-q_{p_{+}^{0}} q_{p_{+}^{3}}^{2} q_{d_{+}^{1}}
\end{aligned}
$$




$$
\begin{aligned}
& +2 q_{p_{+}^{0}} q_{p_{+}^{1}} q_{p_{+}^{2}} q_{d_{+}^{2}}+q_{p_{+}^{0}}^{2} q_{p_{+}^{3}} q_{d_{+}^{2}}+q_{p_{+}^{1}}^{2} q_{p_{+}^{3}} q_{d_{+}^{2}}-q_{p_{+}^{2}}^{2} q_{p_{+}^{3}} q_{d_{+}^{2}} \\
& +q_{p_{+}^{3}}^{3} q_{d_{+}^{2}}+\left(q_{p_{+}^{2}}\left(q_{p_{+}^{0}}^{2}+q_{p_{+}^{1}}^{2}+q_{p_{+}^{2}}^{2}\right)+2 q_{p_{+}^{0}} q_{p_{+}^{1}} q_{p_{+}^{3}}-q_{p_{+}^{2}} q_{p_{+}^{3}}^{2}\right) q_{d_{+}^{3}}, \\
& -q_{p_{-}^{0}} q_{p_{+}^{0}} q_{p_{+}^{1}} q_{d_{-}^{1}}-q_{p_{-}^{3}} q_{p_{+}^{0}} q_{p_{+}^{2}} q_{d_{-}^{1}}-q_{p_{-}^{3}} q_{p_{+}^{1}} q_{p_{+}^{3}} q_{d_{-}^{1}} \\
& +q_{p_{-}^{0}} q_{p_{+}^{2}} q_{p_{+}^{3}} q_{d_{-}^{1}}+q_{p_{-}^{3}} q_{p_{+}^{0}} q_{p_{+}^{1}} q_{d_{-}^{2}}-q_{p_{-}^{0}} q_{p_{+}^{0}} q_{p_{+}^{2}} q_{d_{-}^{2}} \\
& -q_{p_{-}^{0}} q_{p_{+}^{1}} q_{p_{+}^{3}} q_{d_{-}^{2}}-q_{p_{-}^{3}} q_{p_{+}^{2}} q_{p_{+}^{3}} q_{d_{-}^{2}}+q_{p_{-}^{1}}\left(q_{p_{+}^{0}} q_{p_{+}^{1}} q_{d_{-}^{0}}\right. \\
& \left.-q_{p_{+}^{2}} q_{p_{+}^{3}} q_{d_{-}^{0}}+q_{p_{+}^{0}} q_{p_{+}^{2}} q_{d_{-}^{3}}+q_{p_{+}^{1}} q_{p_{+}^{3}} q_{d_{-}^{3}}\right) \\
& +q_{p_{-}^{2}}\left(q_{p_{+}^{0}} q_{p_{+}^{2}} q_{d_{-}^{0}}+q_{p_{+}^{1}} q_{p_{+}^{3}} q_{d_{-}^{0}}-q_{p_{+}^{0}} q_{p_{+}^{1}} q_{d_{-}^{3}}\right. \\
& \left.+q_{p_{+}^{2}} q_{p_{+}^{3}} q_{d_{-}^{3}}\right)-q_{p_{+}^{0}} q_{p_{+}^{1}}^{2} q_{d_{+}^{0}}-q_{p_{+}^{0}} q_{p_{+}^{2}}^{2} q_{d_{+}^{0}}+q_{p_{+}^{0}}^{2} q_{p_{+}^{1}} q_{d_{+}^{1}} \\
& +q_{p_{+}^{1}} q_{p_{+}^{3}}^{2} q_{d_{+}^{1}}+q_{p_{+}^{0}}^{2} q_{p_{+}^{2}} q_{d_{+}^{2}}+q_{p_{+}^{2}}^{2} q_{p_{+}^{3}}^{2} q_{d_{+}^{2}}^{2} \\
& -\left(q_{p_{+}^{1}}^{2}+q_{p_{+}^{2}}^{2}\right) q_{p_{+}^{3}} q_{d_{+}^{3}}, q_{p_{-}^{0}} q_{d_{-}^{0}}+q_{p_{-}^{1}} q_{d_{-}^{1}}+q_{p_{-}^{2}} q_{d_{-}^{2}}+q_{p_{-}^{3}} q_{d_{-}^{3}}, \\
& q_{p_{+}^{0}} q_{d_{+}^{0}}+q_{p_{+}^{1}} q_{d_{+}^{1}}+q_{p_{+}^{2}} q_{d_{+}^{2}}+q_{p_{+}^{3}} q_{d_{+}^{3}}, q_{p_{-}^{0}}^{2}+q_{p_{-}^{1}}^{2}+q_{p_{-}^{2}}^{2} \\
& \left.+q_{p_{-}^{3}}^{2}-1, q_{p_{+}^{0}}^{2}+q_{p_{+}^{1}}^{2}+q_{p_{+}^{2}}^{2}+q_{p_{+}^{3}}^{2}-1\right]^{T}
\end{aligned}
$$

5. S-joint:

$$
\begin{aligned}
\left(q_{p^{i}} ; q_{d^{i}}\right)_{ \pm}^{i=0,1,2,3 \mapsto} \mapsto & {\left[a_{-}\left(q_{p_{-}^{0}}^{2}+q_{p_{-}^{1}}^{2}-q_{p_{-}^{2}}^{2}-q_{p_{-}^{3}}^{2}\right)+2\left(q_{p_{-}^{1}} q_{d_{-}^{0}}-q_{p_{-}^{0}} q_{d_{-}^{1}}+q_{p_{-}^{3}} q_{d_{-}^{2}}\right.\right.} \\
& \left.-q_{p_{-}^{2}} q_{d_{-}^{3}}-q_{p_{+}^{1}} q_{d_{+}^{0}}+q_{p_{+}^{0}} q_{d_{+}^{1}}-q_{p_{+}^{3}} q_{d_{+}^{2}}+q_{p_{+}^{2}} q_{d_{+}^{3}}\right), a_{-} q_{p_{-}^{1}} q_{p_{-}^{2}} \\
& +a_{-} q_{p_{-}^{0}} q_{p_{-}^{3}}+q_{p_{-}^{2}} q_{d_{-}^{0}}-q_{p_{-}^{3}} q_{d_{-}^{1}}-q_{p_{-}^{0}} q_{d_{-}^{2}}+q_{p_{-}^{1}} q_{d_{-}^{3}}-q_{p_{+}^{2}} q_{d_{+}^{0}} \\
& +q_{p_{+}^{3}} q_{d_{+}^{1}}+q_{p_{+}^{0}} q_{d_{+}^{2}}-q_{p_{+}^{1}} q_{d_{+}^{3}}, a_{-} q_{p_{-}^{0}} q_{p_{-}^{2}}-a_{-} q_{p_{-}^{1}} q_{p_{-}^{3}}-q_{p_{-}^{3}} q_{d_{-}^{0}} \\
& -q_{p_{-}^{2}} q_{d_{-}^{1}}+q_{p_{-}^{1}} q_{d_{-}^{2}}+q_{p_{-}^{0}} q_{d_{-}^{3}}+q_{p_{+}^{3}} q_{d_{+}^{0}}+q_{p_{+}^{2}} q_{d_{+}^{1}}-q_{p_{+}^{1}} q_{d_{+}^{2}} \\
& -q_{p_{+}^{0}} q_{d_{+}^{3}}, q_{p_{-}^{0}} q_{d_{-}^{0}}+q_{p_{-}^{1}} q_{d_{-}^{1}}+q_{p_{-}^{2}} q_{d_{-}^{2}}+q_{p_{-}^{3}} q_{d_{-}^{3}}, q_{p_{+}^{0}} q_{d_{+}^{0}} \\
& +q_{p_{+}^{1}} q_{d_{+}^{1}}+q_{p_{+}^{2}} q_{d_{+}^{2}}+q_{p_{+}^{3}} q_{d_{+}^{3}}, q_{p_{-}^{0}}^{2}+q_{p_{-}^{1}}^{2}+q_{p_{-}^{2}}^{2}+q_{p_{-}^{3}}^{2}-1, q_{p_{+}^{0}}^{2} \\
& \left.+q_{p_{+}^{1}}^{2}+q_{p_{+}^{2}}^{2}+q_{p_{+}^{3}}^{2}-1\right]^{T}
\end{aligned}
$$

6. U-joint:

$$
\begin{aligned}
\left(q_{p^{i}} ; q_{d^{i}}\right)_{ \pm}^{i=0,1,2,3} \mapsto[ & {\left[-\cos \varphi_{+}\left(-2\left(-q_{p_{-}^{1}}^{2} q_{p_{+}^{0}} q_{p_{+}^{1}}+q_{p_{-}^{3}}^{2} q_{p_{+}^{0}} q_{p_{+}^{1}}+2 q_{p_{-}^{1}} q_{p_{-}^{3}} q_{p_{+}^{1}} q_{p_{+}^{2}}\right.\right.\right.} \\
& -2 q_{p_{-}^{1}} q_{p_{-}^{3}} q_{p_{+}^{0}} q_{p_{+}^{3}}-q_{p_{-}^{1}}^{2} q_{p_{+}^{2}} q_{p_{+}^{3}}+q_{p_{-}^{3}}^{2} q_{p_{+}^{2}} q_{p_{+}^{3}} \\
& +2 q_{p_{-}^{0}} q_{p_{-}^{2}}\left(q_{p_{+}^{1}} q_{p_{+}^{2}}-q_{p_{+}^{0}} q_{p_{+}^{3}}\right)+q_{p_{-}^{0}}^{2}\left(q_{p_{+}^{0}} q_{p_{+}^{1}}+q_{p_{+}^{2}} q_{p_{+}^{3}}\right) \\
& -q_{p_{-}^{2}}^{2}\left(q_{p_{+}^{0}} q_{p_{+}^{1}}+q_{p_{+}^{2}} q_{p_{+}^{3}}\right)+q_{p_{-}^{2}} q_{p_{-}^{3}}\left(q_{p_{+}^{0}}^{2}-q_{p_{+}^{1}}^{2}+q_{p_{+}^{2}}^{2}\right.
\end{aligned}
$$


199

$$
\begin{aligned}
& \left.\left.-q_{p_{+}^{3}}^{2}\right)+q_{p_{-}^{0}} q_{p_{-}^{1}}\left(-q_{p_{+}^{0}}^{2}+q_{p_{+}^{1}}^{2}-q_{p_{+}^{2}}^{2}+q_{p_{+}^{3}}^{2}\right)\right) \cos \psi_{-} \\
& +\left(q_{p_{-}^{2}}^{2} q_{p_{+}^{0}}^{2}-q_{p_{-}^{3}}^{2} q_{p_{+}^{0}}^{2}+4 q_{p_{-}^{2}} q_{p_{-}^{3}} q_{p_{+}^{0}} q_{p_{+}^{1}}-q_{p_{-}^{2}}^{2} q_{p_{+}^{1}}^{2}+q_{p_{-}^{3}}^{2} q_{p_{+}^{1}}^{2}\right. \\
& +q_{p_{-}^{2}}^{2} q_{p_{+}^{2}}^{2}-q_{p_{-}^{3}}^{2} q_{p_{+}^{2}}^{2}+4 q_{p_{-}^{2}} q_{p_{-}^{3}} q_{p_{+}^{2}} q_{p_{+}^{3}}-q_{p_{-}^{2}}^{2} q_{p_{+}^{3}}^{2} \\
& +q_{p_{-}^{3}}^{2} q_{p_{+}^{3}}^{2}+4 q_{p_{-}^{1}} q_{p_{-}^{2}}\left(q_{p_{+}^{1}} q_{p_{+}^{2}}-q_{p_{+}^{0}} q_{p_{+}^{3}}\right)+4 q_{p_{-}^{0}}\left(q_{p_{-}^{1}} q_{p_{+}^{0}} q_{p_{+}^{1}}\right. \\
& \left.-q_{p_{-}^{3}} q_{p_{+}^{1}} q_{p_{+}^{2}}+q_{p_{-}^{3}} q_{p_{+}^{0}} q_{p_{+}^{3}}+q_{p_{-}^{1}} q_{p_{+}^{2}} q_{p_{+}^{3}}\right)+q_{p_{-}^{0}}^{2}\left(q_{p_{+}^{0}}^{2}\right. \\
& \left.\left.\left.-q_{p_{+}^{1}}^{2}+q_{p_{+}^{2}}^{2}-q_{p_{+}^{3}}^{2}\right)+q_{p_{-}^{1}}^{2}\left(-q_{p_{+}^{0}}^{2}+q_{p_{+}^{1}}^{2}-q_{p_{+}^{2}}^{2}+q_{p_{+}^{3}}^{2}\right)\right) \sin \psi_{-}\right) \\
& +2 \sin \varphi_{+}\left(\left(-q_{p_{-}^{2}}^{2} q_{p_{+}^{0}} q_{p_{+}^{2}}+q_{p_{-}^{3}}^{2} q_{p_{+}^{0}} q_{p_{+}^{2}}-2 q_{p_{-}^{2}} q_{p_{-}^{3}} q_{p_{+}^{1}} q_{p_{+}^{2}}\right.\right. \\
& -2 q_{p_{-}^{2}} q_{p_{-}^{3}} q_{p_{+}^{0}} q_{p_{+}^{3}}+q_{p_{-}^{2}}^{2} q_{p_{+}^{1}} q_{p_{+}^{3}}-q_{p_{-}^{3}}^{2} q_{p_{+}^{1}} q_{p_{+}^{3}}+2 q_{p_{-}^{0}} q_{p_{-}^{1}}\left(q_{p_{+}^{1}} q_{p_{+}^{2}}\right. \\
& \left.+q_{p_{+}^{0}} q_{p_{+}^{3}}\right)+q_{p_{-}^{0}}^{2}\left(q_{p_{+}^{0}} q_{p_{+}^{2}}-q_{p_{+}^{1}} q_{p_{+}^{3}}\right)+q_{p_{-}^{1}}^{2}\left(-q_{p_{+}^{0}} q_{p_{+}^{2}}\right. \\
& \left.+q_{p_{+}^{1}} q_{p_{+}^{3}}\right)+q_{p_{-}^{0}} q_{p_{-}^{2}}\left(-q_{p_{+}^{0}}^{2}-q_{p_{+}^{1}}^{2}+q_{p_{+}^{2}}^{2}+q_{p_{+}^{3}}^{2}\right) \\
& \left.+q_{p_{-}^{1}} q_{p_{-}^{3}}\left(-q_{p_{+}^{0}}^{2}-q_{p_{+}^{1}}^{2}+q_{p_{+}^{2}}^{2}+q_{p_{+}^{3}}^{2}\right)\right) \cos \psi_{-}+\left(-2 q_{p_{-}^{2}} q_{p_{-}^{3}} q_{p_{+}^{0}} q_{p_{+}^{2}}\right. \\
& +q_{p_{-}^{2}}^{2} q_{p_{+}^{1}} q_{p_{+}^{2}}-q_{p_{-}^{3}}^{2} q_{p_{+}^{1}} q_{p_{+}^{2}}+q_{p_{-}^{2}}^{2} q_{p_{+}^{0}} q_{p_{+}^{3}}-q_{p_{-}^{3}}^{2} q_{p_{+}^{0}} q_{p_{+}^{3}} \\
& +2 q_{p_{-}^{2}} q_{p_{-}^{3}} q_{p_{+}^{1}} q_{p_{+}^{3}}+q_{p_{-}^{0}}^{2}\left(q_{p_{+}^{1}} q_{p_{+}^{2}}+q_{p_{+}^{0}} q_{p_{+}^{3}}\right) \\
& -q_{p_{-}^{1}}^{2}\left(q_{p_{+}^{1}} q_{p_{+}^{2}}+q_{p_{+}^{0}} q_{p_{+}^{3}}\right)+q_{p_{-}^{0}} q_{p_{-}^{3}}\left(-q_{p_{+}^{0}}^{2}-q_{p_{+}^{1}}^{2}+q_{p_{+}^{2}}^{2}\right. \\
& \left.+q_{p_{+}^{3}}^{2}\right)+q_{p_{-}^{1}}\left(-2 q_{p_{-}^{0}} q_{p_{+}^{0}} q_{p_{+}^{2}}+2 q_{p_{-}^{0}} q_{p_{+}^{1}} q_{p_{+}^{3}}\right. \\
& \left.\left.\left.+q_{p_{-}^{2}}\left(q_{p_{+}^{0}}^{2}+q_{p_{+}^{1}}^{2}-q_{p_{+}^{2}}^{2}-q_{p_{+}^{3}}^{2}\right)\right)\right) \sin \psi_{-}\right), a_{-}\left(q_{p_{-}^{0}}^{2}+q_{p_{-}^{1}}^{2}\right. \\
& \left.-q_{p_{-}^{2}}^{2}-q_{p_{-}^{3}}^{2}\right)-2\left(d_{+} q_{p_{+}^{0}} q_{p_{+}^{2}}+d_{+} q_{p_{+}^{1}} q_{p_{+}^{3}}-q_{p_{-}^{1}} q_{d_{-}^{0}}+q_{p_{-}^{0}} q_{d_{-}^{1}}\right. \\
& \left.-q_{p_{-}^{3}} q_{d_{-}^{2}}+q_{p_{-}^{2}} q_{d_{-}^{3}}+q_{p_{+}^{1}} q_{d_{+}^{0}}-q_{p_{+}^{0}} q_{d_{+}^{1}}+q_{p_{+}^{3}} q_{d_{+}^{2}}-q_{p_{+}^{2}} q_{d_{+}^{3}}\right), \\
& a_{-} q_{p_{-}^{1}} q_{p_{-}^{2}}+a_{-} q_{p_{-}^{0}} q_{p_{-}^{3}}+d_{+} q_{p_{+}^{0}} q_{p_{+}^{1}}-d_{+} q_{p_{+}^{2}} q_{p_{+}^{3}} \\
& +q_{p_{-}^{2}} q_{d_{-}^{0}}-q_{p_{-}^{3}} q_{d_{-}^{1}}-q_{p_{-}^{0}} q_{d_{-}^{2}}+q_{p_{-}^{1}} q_{d_{-}^{3}}-q_{p_{+}^{2}} q_{d_{+}^{0}}+q_{p_{+}^{3}} q_{d_{+}^{1}} \\
& +q_{p_{+}^{0}} q_{d_{+}^{2}}-q_{p_{+}^{1}} q_{d_{+}^{3}}, a_{-}\left(-2 q_{p_{-}^{0}} q_{p_{-}^{2}}+2 q_{p_{-}^{1}} q_{p_{-}^{3}}\right)+d_{+}\left(-q_{p_{+}^{0}}^{2}\right. \\
& \left.+q_{p_{+}^{1}}^{2}+q_{p_{+}^{2}}^{2}-q_{p_{+}^{3}}^{2}\right)+2\left(q_{p_{-}^{3}} q_{d_{-}^{0}}+q_{p_{-}^{2}} q_{d_{-}^{1}}-q_{p_{-}^{1}} q_{d_{-}^{2}}\right. \\
& \left.-q_{p_{-}^{0}} q_{d_{-}^{3}}-q_{p_{+}^{3}} q_{d_{+}^{0}}-q_{p_{+}^{2}} q_{d_{+}^{1}}+q_{p_{+}^{1}} q_{d_{+}^{2}}+q_{p_{+}^{0}} q_{d_{+}^{3}}\right), q_{p_{-}^{0}} q_{d_{-}^{0}} \\
& +q_{p_{-}^{1}} q_{d_{-}^{1}}+q_{p_{-}^{2}} q_{d_{-}^{2}}+q_{p_{-}^{3}} q_{d_{-}^{3}}, q_{p_{+}^{0}} q_{d_{+}^{0}}+q_{p_{+}^{1}} q_{d_{+}^{1}} \\
& +q_{p_{+}^{2}} q_{d_{+}^{2}}+q_{p_{+}^{3}} q_{d_{+}^{3}}, q_{p_{-}^{0}}^{2}+q_{p_{-}^{1}}^{2}+q_{p_{-}^{2}}^{2}+q_{p_{-}^{3}}^{2}-1, q_{p_{+}^{0}}^{2} \\
& \left.+q_{p_{+}^{1}}^{2}+q_{p_{+}^{2}}^{2}+q_{p_{+}^{3}}^{2}-1\right]^{T}
\end{aligned}
$$




\section{Appendix B}

\section{Singularities and Grashof-type}

\section{conditions for 3-RRR PPM}

To illustrate the derivation of the conditions in (7.4) eight of the $(4 \times 4)$ minors are shown. In the following, we write $s_{i j}=\sin \left(\theta_{i}-\theta_{j}\right)$ and similarly for cosine.

$$
\begin{aligned}
& S_{1}=\left[\begin{array}{cccc}
-l_{1} s_{1} & l_{2} s_{2} & 0 & -l_{4} s_{4} \\
l_{1} c_{1} & -l_{2} c_{2} & 0 & l_{4} c_{4} \\
0 & -l_{2} s_{2} & l_{3} s_{3} & 0 \\
0 & l_{2} c_{2} & -l_{3} c_{3} & 0
\end{array}\right], \quad S_{2}=\left[\begin{array}{cccc}
-l_{1} s_{1} & l_{2} s_{2} & 0 & l_{5} s_{5} \\
l_{1} c_{1} & -l_{2} c_{2} & 0 & -l_{5} c_{5} \\
0 & -l_{2} s_{2} & l_{3} s_{3} & -l_{5} s_{5} \\
0 & l_{2} c_{2} & -l_{3} c_{3} & l_{5} c_{5}
\end{array}\right] \\
& S_{3}=\left[\begin{array}{cccc}
-l_{1} s_{1} & l_{2} s_{2} & 0 & 0 \\
l_{1} c_{1} & -l_{2} c_{2} & 0 & 0 \\
0 & -l_{2} s_{2} & l_{3} s_{3} & l_{6} s_{6} \\
0 & l_{2} c_{2} & -l_{3} c_{3} & -l_{6} c_{6}
\end{array}\right], \quad S_{4}=\left[\begin{array}{cccc}
-l_{1} s_{1} & l_{2} s_{2} & 0 & -a_{1} s_{7} \\
l_{1} c_{1} & -l_{2} c_{2} & 0 & a_{1} c_{7} \\
0 & -l_{2} s_{2} & l_{3} s_{3} & m_{34} \\
0 & l_{2} c_{2} & -l_{3} c_{3} & m_{44}
\end{array}\right], \\
& S_{5}=\left[\begin{array}{cccc}
-l_{1} s_{1} & l_{2} s_{2} & -l_{4} s_{4} & l_{5} s_{5} \\
l_{1} c_{1} & -l_{2} c_{2} & l_{4} c_{4} & -l_{5} c_{5} \\
0 & -l_{2} s_{2} & 0 & -l_{5} s_{5} \\
0 & l_{2} c_{2} & 0 & l_{5} c_{5}
\end{array}\right], \quad S_{6}=\left[\begin{array}{cccc}
-l_{1} s_{1} & l_{2} s_{2} & -l_{4} s_{4} & 0 \\
l_{1} c_{1} & -l_{2} c_{2} & l_{4} c_{4} & 0 \\
0 & -l_{2} s_{2} & 0 & l_{6} s_{6} \\
0 & l_{2} c_{2} & 0 & -l_{6} c_{6}
\end{array}\right] \text {, } \\
& S_{7}=\left[\begin{array}{cccc}
-l_{1} s_{1} & l_{2} s_{2} & -l_{4} s_{4} & -a_{1} s_{7} \\
l_{1} c_{1} & -l_{2} c_{2} & l_{4} c_{4} & a_{1} c_{7} \\
0 & -l_{2} s_{2} & 0 & m_{34} \\
0 & l_{2} c_{2} & 0 & m_{44}
\end{array}\right], \quad S_{8}=\left[\begin{array}{cccc}
-l_{1} s_{1} & l_{2} s_{2} & l_{5} s_{5} & 0 \\
l_{1} c_{1} & -l_{2} c_{2} & -l_{5} c_{5} & 0 \\
0 & -l_{2} s_{2} & -l_{5} s_{5} & l_{6} s_{6} \\
0 & l_{2} c_{2} & l_{5} c_{5} & -l_{6} c_{6}
\end{array}\right]
\end{aligned}
$$


where $m_{34}=a_{1} s_{7}-a_{2} s_{\alpha, 7}$ and $m_{44}=-a_{1} c_{7}+a_{2} c_{\alpha, 7}$. Their determinants, together with the other 27 which have similar shapes, must vanish simultaneously for a singular point of $F$ and

$$
\begin{aligned}
& \operatorname{det} S_{1}=-\left(s_{23}\right)\left(s_{14}\right) l_{1} l_{2} l_{3} l_{4}, \\
& \operatorname{det} S_{2}=\left(s_{13}\right)\left(s_{25}\right) l_{1} l_{2} l_{3} l_{5}, \\
& \operatorname{det} S_{3}=-\left(s_{12}\right)\left(s_{36}\right) l_{1} l_{2} l_{3} l_{6}, \\
& \operatorname{det} S_{4}=\left[-a_{1}\left(s_{13}\right)\left(s_{27}\right)+a_{2}\left(s_{12}\right)\left(s_{\alpha, 3,7}\right)\right] l_{1} l_{2} l_{3}, \\
& \operatorname{det} S_{5}=-\left(s_{14}\right)\left(s_{25}\right) l_{1} l_{2} l_{4} l_{5}, \\
& \operatorname{det} S_{6}=\left(s_{14}\right)\left(s_{26}\right) l_{1} l_{2} l_{4} l_{6}, \\
& \operatorname{det} S_{7}=\left(s_{14}\right)\left[a_{1}\left(s_{27}\right)+a_{2}\left(s_{\alpha, 2,7}\right)\right] l_{1} l_{2} l_{4}, \\
& \operatorname{det} S_{8}=-\left(s_{16}\right)\left(s_{25}\right) l_{1} l_{2} l_{5} l_{6},
\end{aligned}
$$

where $s_{\alpha, 3,7}=\sin \left(\alpha-\theta_{3}+\theta_{7}\right)$ and $s_{\alpha, 2,7}=\sin \left(\alpha-\theta_{2}+\theta_{7}\right)$. Assume that $l_{k}>0, k=1, \ldots 6$, then it is clear from (B.1) that, for example

- $\operatorname{det} S_{1}=0 \Longleftrightarrow \theta_{2}=\theta_{3} \bmod \pi$ or $\theta_{1}=\theta_{4} \bmod \pi$

- $\operatorname{det} S_{2}=0 \Longleftrightarrow \theta_{1}=\theta_{3} \bmod \pi$ or $\theta_{2}=\theta_{5} \bmod \pi$

- $\operatorname{det} S_{3}=0 \Longleftrightarrow \theta_{1}=\theta_{2} \bmod \pi$ or $\theta_{3}=\theta_{6} \bmod \pi$

- $\operatorname{det} S_{5}=0 \Longleftrightarrow \theta_{1}=\theta_{4} \bmod \pi$ or $\theta_{2}=\theta_{5} \bmod \pi$

- $\operatorname{det} S_{6}=0 \Longleftrightarrow \theta_{1}=\theta_{2} \bmod \pi$ or $\theta_{2}=\theta_{6} \bmod \pi$

- $\operatorname{det} S_{8}=0 \Longleftrightarrow \theta_{1}=\theta_{6} \bmod \pi$ or $\theta_{2}=\theta_{5} \bmod \pi$

From these and similar solutions for all the minors we deduce that one of the sets of equalities in (7.4) must hold. In all such cases, expressions such as those in $\operatorname{det} S_{4}, \operatorname{det} S_{7}, \operatorname{det} S_{9}, \operatorname{det} S_{10}$ also vanish, ensuring singularity. The full set of conditions can be found in [110].

Let us consider a configuration where $\theta_{1}=\theta_{2}=\theta_{4}=\theta_{5}=\theta_{7} \bmod \pi$, satisfying singularity conditions in (7.4a) and substitute it into $F\left(x_{i}, y_{i}, \theta_{i}\right)=$ $\mathbf{0}$ where $F$ is given in (7.2). It follows that $c_{j}= \pm c_{i}, s_{j}= \pm s_{i}, i, j=$ $1,2,4,5,7$. Eliminating $x_{i}, y_{i}, i=1, \ldots, 7$ from the first 14 equations, we end 
up with the following relations for the design parameters and pose rotations in Figure 7.1

$$
\begin{aligned}
& \mu c_{1}=b_{1}, \\
& \mu s_{1}=0, \\
& \xi c_{1}=a_{2} s_{1} s_{\alpha}+l_{3} c_{3}+l_{6} c_{6}+b_{2} c_{\beta}, \\
& \xi s_{1}=-a_{2} c_{1} s_{\alpha}+l_{3} s_{3}+l_{6} s_{6}+b_{2} s_{\beta},
\end{aligned}
$$

where $\mu= \pm a_{1} \pm l_{1} \pm l_{4} \pm l_{2} \pm l_{5}$ and $\xi= \pm a_{2} c_{\alpha} \pm l_{1} \pm l_{4}$. Assuming $b_{1}>0$, equations (B.2a) and (B.2b) imply that $\mu \neq 0$ and $\theta_{1}=0$ or $\pi$ and thus the generalised Grashof condition $\mu=b_{1}$ in equation (7.5) is readily obtained.

At the same time, equations (B.2c) and (B.2d) imply that, in such a singular configuration, $\theta_{3}, \theta_{6}$ may have several solutions, so the limb $l_{3} l_{6}$ may be positioned in different configurations as illustrated Figure 7.2a. Mathematically speaking, given $\theta_{1}$ as above, we can write equations (B.2c) and (B.2d) as linear equations in $c_{3}, s_{3}, c_{6}, s_{6}$ together with the identities $c_{3}^{2}+s_{3}^{2}=1, c_{6}^{2}+s_{6}^{2}=1$

$$
\begin{aligned}
l_{3} c_{3}+l_{6} c_{6}+b_{2} c_{\beta}-\xi & =0, \\
l_{3} s_{3}+l_{6} s_{6}+b_{2} s_{\beta}-a_{2} s_{\alpha} & =0
\end{aligned}
$$

This gives four equations, two linear and two quadratic, in the four variables $c_{3}, s_{3}, c_{6}, s_{6}$ and hence can have up to four distinct (complex) solutions. One way to view this is that solving equations (B.3a) and (B.3b) for $c_{3}, s_{3}$ and substituting them into $c_{3}^{2}+s_{3}^{2}=1$ gives

$$
\left(l_{6} c_{6}+b_{2} c_{\beta}-\xi\right)^{2}+\left(l_{6} s_{6}+b_{2} s_{\beta}-a_{2} s_{\alpha}\right)^{2}=l_{3}^{2}
$$

which represents a circle. This can only intersect the circle $c_{6}^{2}+s_{6}^{2}=1$ in at most two real points, the other solutions being the complex circular points at infinity. 
Finally, let us consider a configuration where $\theta_{i}=\eta_{i} \pi, \eta_{i} \in \mathbb{Z}, i=1, \ldots, 6$ satisfying singularity set in (7.4d) and substitute the conditions into (7.2). It follows that $c_{j}= \pm c_{i}, s_{j}= \pm s_{i}, i, j=1, \ldots, 6$. By setting $F=\mathbf{0}$ and eliminating $x_{i}, y_{i}, i=1, \ldots, 7$, we end up with the following four linear relations in $c_{1}, s_{1}, c_{7}, s_{7}$ :

$$
\begin{aligned}
a_{1} c_{7}-\zeta c_{1} & =b_{1}, \\
a_{1} s_{7}-\zeta s_{1} & =0, \\
a_{2} c_{\alpha} c_{7}-a_{2} s_{\alpha} s_{7}-\vartheta c_{1} & =b_{2} c_{\beta}, \\
a_{2} s_{\alpha} c_{7}+a_{2} c_{\alpha} s_{7}-\vartheta s_{1} & =b_{2} s_{\beta}
\end{aligned}
$$

where $\zeta= \pm l_{1} \pm l_{4} \pm l_{2} \pm l_{5}$ and $\vartheta= \pm l_{1} \pm l_{4} \pm l_{3} \pm l_{6}$. Solving these and combining with the identities $c_{1}^{2}+s_{1}^{2}=c_{7}^{2}+s_{7}^{2}=1$ gives the Grashof-type conditions (7.6). We conjecture that these equations equate three geometric expressions relating to the three closed-loop chains in Figure 7.1, that enable a feasible configuration in which the three limbs are parallel. 


\section{Bibliography}

[1] C. Gosselin and J. Angeles, "Singularity analysis of closed-loop kinematic chains," IEEE Transactions on Robotics and Automation, vol. 6, no. 3, pp. 281-290, 1990.

[2] D. Zlatanov, R. G. Fenton, and B. Benhabib, "Singularity analysis of mechanisms and robots via a motion-space model of the instantaneous kinematics," in Robotics and Automation, 1994. Proceedings., 1994 IEEE International Conference on, pp. 980-985, IEEE, 1994.

[3] F. Park and J. W. Kim, "Manipulability of closed kinematic chains," Journal of Mechanical Design, vol. 120, no. 4, pp. 542-548, 1998.

[4] F. Grashof, Theoretische Maschinenlehre, vol. 3. L. Voss, 1890.

[5] A. André-Marie, "Essai sur la philosophie des sciences," Paris: Chez Bachelier, 1834.

[6] T. Koetsier, "The case of kinematics, the genesis of a discipline," in Explorations in the History of Machines and Mechanisms, pp. 491-504, Springer, 2012.

[7] W. R. Hamilton, Elements of Quaternions. Longmans, Green, \& Company, 1866.

[8] N. L. White, "Grassmann - cayley algebra and robotics," Journal of Intelligent and Robotic Systems, vol. 11, no. 1-2, pp. 91-107, 1994. 
[9] W. K. Clifford, A preliminary sketch of biquaternions. Proceedings of the London Mathematical Society, 1873.

[10] R. S. Ball, A Treatise on the Theory of Screws. Cambridge University Press, 1998.

[11] E. Study, "Of the movements and redistributions: I. and II. treatise," Mathematical Annals, vol. 39, pp. 441-565, 1891.

[12] D. L. Peiper, "The kinematics of manipulators under computer control," tech. rep., STANFORD UNIV CA DEPT OF COMPUTER SCIENCE, 1968.

[13] R. S. Hartenberg and J. Denavit, "A kinematic notation for lower pair mechanisms based on matrices," Journal of applied mechanics, vol. 77, no. 2, pp. 215-221, 1955.

[14] V. Gough, "Contribution to discussion of papers on research in automobile stability, control and tyre performance," Proc. of Auto Div. Inst. Mech. Eng., vol. 171, pp. 392-395, 1957.

[15] D. Stewart, "A platform with six degrees of freedom," Proceedings of the institution of mechanical engineers, vol. 180, no. 1, pp. 371-386, 1965.

[16] K. H. Hunt, Kinematic Geometry of Mechanisms, vol. 7. Oxford University Press, USA, 1978.

[17] J. Phillips, "Introducing screw theory, volume 1 of freedom in machinery," 1984.

[18] J.-P. Merlet, "Singular configurations of parallel manipulators and Grassmann geometry," International Journal of Robotics Research, vol. 8, no. 5, pp. 45-56, 1989. 
[19] M. Husty and P. Zsombor-Murray, "A special type of singular stewartgough platform," in Advances in robot kinematics and computational geometry, pp. 449-458, Springer, 1994.

[20] K. Sugimoto, J. Duffy, and K. Hunt, "Special configurations of spatial mechanisms and robot arms," Mechanism and Machine Theory, vol. 17, no. 2, pp. 119-132, 1982.

[21] F. Litvin, Z. Yi, V. P. Castelli, and C. Innocenti, "Singularities, configurations, and displacement functions for manipulators," International Journal of Robotics Research, vol. 5, no. 2, pp. 52-65, 1986.

[22] H. Zhang, "Kinematic stability of robot manipulators under force control," in Proceedings, 1989 International Conference on Robotics and Automation, pp. 80-85, IEEE, 1989.

[23] C. J. Damaren, "Modal properties and control system design for twolink flexible manipulators," The International Journal of Robotics Research, vol. 17, no. 6, pp. 667-678, 1998.

[24] B. Fei, W. S. Ng, S. Chauhan, and C. K. Kwoh, "The safety issues of medical robotics," Reliability Engineering \& System Safety, vol. 73, no. 2, pp. 183-192, 2001.

[25] J. Kieffer and J. Lenarcic, "On the exploitation of mechanical advantage near robot singularities," Informatica, vol. 18, no. 3, pp. 315-323, 1994.

[26] J. Carretero, M. Nahon, C. Gosselin, and B. Buckham, "Kinematic analysis of a three-dof parallel mechanism for telescope applications," in Proceedings of the 1997 ASME Design Engineering Technical Conferences, 1997. 
[27] H. M. Daniali, P. Zsombor-Murray, and J. Angeles, "Singularity analysis of planar parallel manipulators," Mechanism and Machine Theory, vol. 30, no. 5, pp. 665-678, 1995.

[28] I. Bonev and C. Gosselin, "Singularity analysis of 3-dof planar parallel mechanisms via screw theory," Journal of Mechanical Design, vol. 125, pp. 573-581, 2003.

[29] P. S. Donelan, "Singularity-theoretic methods in robot kinematics," Robotica, vol. 25, no. 6, pp. 641-659, 2007.

[30] D. E. Whitney, "Resolved motion rate control of manipulators and human prostheses," IEEE Transactions on man-machine systems, vol. 10, no. 2, pp. 47-53, 1969.

[31] V. Guillemin and A. Pollack, Differential Topology, vol. 370. American Mathematical Society, 2010.

[32] D. K. Pai and M. C. Leu, "Genericity and singularities of robot manipulators," IEEE Transactions on Robotics and Automation, vol. 8, no. 5, pp. 545-559, 1992.

[33] M. Golubitsky and V. Guillemin, Stable Mappings and Their Singularities. Graduate Texts in Mathematics, Springer, 2012.

[34] K. Tchon,, "Differential topology of the inverse kinematic problem for redundant robot manipulators," International Journal of Robotics Research, vol. 10, no. 5, pp. 492-504, 1991.

[35] K. Tchoń and R. Muszyński, "Singularities of nonredundant robot kinematics," International Journal of Robotics Research, vol. 16, no. 1, pp. 60-76, 1997.

[36] P. S. Donelan, "Generic properties in euclidean kinematics," Acta Applicandae Mathematica, vol. 12, no. 3, pp. 265-286, 1988. 
[37] C. Gibson, "Kinematic singularities - a new mathematical tool," in Proc. 3rd Int. Workshop on Advances in Robot Kinematics, Ferrara, Italy, pp. 209-215, 1992.

[38] C. Gibson and C. Hobbs, "Simple singularities of space curves," in Mathematical Proceedings of the Cambridge Philosophical Society, vol. 113, pp. 297-310, Cambridge University Press, 1993.

[39] P. S. Donelan, C. G. Gibson, and W. Hawes, "Trajectory singularities of general planar motions," Proceedings of the Royal Society of Edinburgh Section A: Mathematics, vol. 129, no. 1, pp. 37-55, 1999.

[40] J. W. Burdick, "A classification of 3R regional manipulator singularities and geometries," Mechanism and Machine Theory, vol. 30, no. 1, pp. 71-89, 1995.

[41] P. Wenger and J. El Omri, "Changing posture for cuspidal robot manipulators," in Robotics and Automation, 1996. Proceedings., 1996 IEEE International Conference on, vol. 4, pp. 3173-3178, IEEE, 1996.

[42] P. Wenger, "Classification of 3R positioning manipulators," Journal of Mechanical Design, vol. 120, no. 2, pp. 327-332, 1998.

[43] A. Karger, "Singularity analysis of serial robot-manipulators," Journal of Mechanical Design, vol. 118, no. 4, pp. 520-525, 1996.

[44] J. Lerbet, "Analytic geometry and singularities of mechanisms," ZAMM Journal of Applied Mathematics and Mechanics, vol. 78, no. 10, pp. 687-694, 1998.

[45] J. Lerbet and K. Hao, "Kinematics of mechanisms to the second orderapplication to the closed mechanisms," Acta Applicandae Mathematica, vol. 59, no. 1, pp. 1-19, 1999. 
[46] R. W. Brockett, "Robotic manipulators and the product of exponentials formula," in Mathematical theory of networks and systems, pp. 120-129, Springer, 1984.

[47] C. G. Gibson and P. E. Newstead, "On the geometry of the planar 4-bar mechanism," Acta Applicandae Mathematicae, vol. 7, no. 2, pp. 113$135,1986$.

[48] C. G. Gibson and D. Marsh, "On the linkage varieties of Watt 6bar mechanisms-I. basic geometry," Mechanism and Machine Theory, vol. 24, no. 2, pp. 105-113, 1989.

[49] A. Karger and M. Husty, "Classification of all self-motions of the original Stewart-Gough platform," Computer-Aided Design, vol. 30, no. 3, pp. 205-215, 1998.

[50] C. Torras, F. Thomas, and M. Alberich-Carramiñana, "Stratifying the singularity loci of a class of parallel manipulators," IEEE Transactions on Robotics, vol. 22, no. 1, pp. 23-32, 2006.

[51] P. Ben-Horin and M. Shoham, "Application of Grassmann-Cayley algebra to geometrical interpretation of parallel robot singularities," International Journal of Robotics Research, vol. 28, no. 1, pp. 127-141, 2009 .

[52] A. Mueller, "On the terminology and geometric aspects of redundant parallel manipulators," Robotica, vol. 31, no. 1, pp. 137-147, 2013.

[53] O. Bohigas, M. Manubens, and L. Ros, Singularities of robot mechanisms: Numerical computation and avoidance path planning, vol. 41. Springer, 2016.

[54] F. Park and J. W. Kim, "Singularity analysis of closed kinematic chains," Journal of Mechanical Design, vol. 121, no. 1, pp. 32-38, 1999. 
[55] D. Zlatanov, I. A. Bonev, and C. M. Gosselin, "Constraint singularities of parallel mechanisms," in Robotics and Automation, 2002. Proceedings. ICRA'02. IEEE International Conference on, vol. 1, pp. 496-502, IEEE, 2002.

[56] N. Shvalb, M. Shoham, H. Bamberger, and D. Blanc, "Topological and kinematic singularities for a class of parallel mechanisms," Mathematical Problems in Engineering, 2009.

[57] C. Chen, "The order of local mobility of mechanisms," Mechanism and Machine Theory, vol. 46, no. 9, pp. 1251-1264, 2011.

[58] A. Müller, "On the manifold property of the set of singularities of kinematic mappings: Genericity conditions," Journal of Mechanisms and Robotics, vol. 4, no. 1, p. 011006, 2012.

[59] A. Müller, "Local kinematic analysis of closed-loop linkages-mobility, singularities, and shakiness," Journal of Mechanisms and Robotics, vol. 8, no. 4, p. 041013, 2016.

[60] A. Müller, "Higher-order analysis of kinematic singularities of lower pair linkages and serial manipulators," Journal of Mechanisms and Robotics, vol. 10, no. 1, p. 011008, 2018.

[61] S. Piipponen and J. Tuomela, "Algebraic analysis of kinematics of multibody systems," Mechanical Sciences, vol. 4, no. 1, pp. 33-47, 2013.

[62] G. A. Kramer, Solving Geometric Constraint Systems: a Case Study in Kinematics. MIT press, 1992.

[63] G. Mozzi, Discorso Matematico sopra il Rotamento Momentaneo dei Corpi. Donate Campo, 1763. 
[64] M. Chasles, "A note on the general properties of a system of two similar bodies arbitrarily positioned in space; and on the finite or infinitely small displacement of an unconstrained solid body," Bulletin des Sciences Mathématiques, Férussac, vol. 14, pp. 321-26, 1830.

[65] S. V. Amirinezhad and P. Donelan, "Kinematic constraint maps, C-space singularities and generalised Grashof conditions," in 2017 IDETC/CIE Conference, pp. V05BT08A071, 10pp, ASME, 2017.

[66] S. V. Amirinezhad and P. Donelan, "Kinematic singularities of a 3-dof planar geared robot manipulator," in Advances in Robot Kinematics 2016, pp. 441-449, Springer, 2018.

[67] S. V. Amirinezhad and P. Donelan, "Kinematic constraint maps and c-space singularities for planar mechanisms with prismatic joints," in International Symposium on Advances in Robot Kinematics, pp. 212220, Springer, 2018.

[68] S. V. Amirinezhad, P. Donelan, and A. Müller, "Transversality and its applications to kinematics," in International Symposium on Advances in Robot Kinematics, pp. 221-229, Springer, 2018.

[69] S. V. Amirinezhad and P. Donelan, "Input and output singularities for parallel manipulators," in The 15th IFToMM World Congress, Krakow, Poland, to appear, 2019.

[70] J. M. Selig, Geometric Fundamentals of Robotics. Springer Science \& Business Media, 2004.

[71] W. R. Scott, Group Theory. Courier Corporation, 2012.

[72] C. G. Gibson, Singular Points of Smooth Mappings, vol. 25. Pitman Publishing, 1979. 
[73] R. Gilmore, Lie Groups, Lie Algebras, and some of Their Applications. Dover, 2012.

[74] A. L. Onishchik, E. Vinberg, and V. Minachin, Lie Groups and Lie Algebras. Springer, 1993.

[75] B. O'Neill, Elementary Differential Geometry. Academic Press, 1966.

[76] J. Stillwell, Naive Lie Theory. Springer Science \& Business Media, 2008.

[77] A. I. R. Galarza and J. Seade, Introduction to Classical Geometries. Springer Science \& Business Media, 2007.

[78] J. J. Uicker, B. Ravani, and P. N. Sheth, Matrix Methods in the Design Analysis of Mechanisms and Multibody Systems. Cambridge University Press, 2013.

[79] J. M. McCarthy, Introduction to Theoretical Kinematics. MIT press, 1990.

[80] R. M. Murray, A Mathematical Introduction to Robotic Manipulation. CRC press, 2017.

[81] E. G. Hemingway and O. M. O'Reilly, "Perspectives on euler angle singularities, gimbal lock, and the orthogonality of applied forces and applied moments," Multibody System Dynamics, vol. 44, no. 1, pp. 31$56,2018$.

[82] B. A. Rosenfeld, A History of Non-Euclidean Geometry: Evolution of the Concept of a Geometric Space, vol. 12. Springer Science \& Business Media, 2012.

[83] M. Pfurner, Analysis of spatial serial manipulators using kinematic mapping. PhD thesis, Universität Innsbruck, 2006. 
[84] D. Klawitter, Clifford Algebras: Geometric Modelling and Chain Geometries with Application in Kinematics. Springer, 2014.

[85] E. W. Weisstein, "Fiber." http://mathworld.wolfram.com/Fiber.html.

[86] L.-W. Tsai, "The kinematics of spatial robotic bevel-gear trains," IEEE Journal on Robotics and Automation, vol. 4, no. 2, pp. 150-156, 1988.

[87] I. Talpasanu, T. Yih, and P. Simionescu, "Application of matroid method in kinematic analysis of parallel axes epicyclic gear trains," Journal of Mechanical Design, vol. 128, no. 6, pp. 1307-1314, 2006.

[88] S. V. Amirinezhad and M. K. Uyguroğlu, "Kinematic analysis of geared robotic mechanism using matroid and T-T graph methods," Mechanism and Machine Theory, vol. 88, pp. 16-30, 2015.

[89] M. Spong and M. Vidyasagar, "Robot dynamics and control," 1989.

[90] E. J. Haug, Computer Aided Kinematics and Dynamics of Mechanical Systems, vol. 1. Allyn and Bacon Boston, 1989.

[91] J. J. Craig, Introduction to Robotics: Mechanics and Control, vol. 3. Pearson/Prentice Hall Upper Saddle River, NJ, USA:, 2005.

[92] M. L. Husty and H.-P. Schröcker, "Algebraic geometry and kinematics," in Nonlinear Computational Geometry, pp. 85-107, Springer, 2009 .

[93] M. Spivak, Calculus on manifolds: a modern approach to classical theorems of advanced calculus. WA Benjamin, Inc., New York-Amsterdam, 1965.

[94] M. Daher and P. Donelan, "Invariant properties of the DenavitHartenberg parameters," in Interdisciplinary Applications of Kinematics, pp. 43-51, Springer, 2015. 
[95] K.-L. Ting and Y.-W. Liu, "Rotatability laws for N-bar kinematic chains and their proof," Journal of Mechanical Design, vol. 113, no. 1, pp. 32-39, 1991.

[96] D. K. Pai and M. C. Leu, "Generic singularities of robot manipulators," in Proceedings, IEEE International Conference on Robotics and Automation, pp. 738-744, IEEE, 1989.

[97] K. Tchon and R. Muszynski, "Singular inverse kinematic problem for robotic manipulators: A normal form approach," IEEE Transactions on Robotics and Automation, vol. 14, no. 1, pp. 93-104, 1998.

[98] P. Donelan, "Genericity conditions for serial manipulators," Advances in Robot Kinematics: Analysis and Design, pp. 185-192, 2008.

[99] A. Müller, "Generic mobility of rigid body mechanisms," Mechanism and Machine Theory, vol. 44, no. 6, pp. 1240-1255, 2009.

[100] K. H. Hunt and P. R. McAree, "The octahedral manipulator: geometry and mobility," International Journal of Robotics Research, vol. 17, no. 8, pp. 868-885, 1998.

[101] B. M. St-Onge and C. M. Gosselin, "Singularity analysis and representation of the general Gough-Stewart platform," International Journal of Robotics Research, vol. 19, no. 3, pp. 271-288, 2000.

[102] H. Li, C. M. Gosselin, M. J. Richard, and B. M. St-Onge, "Analytic form of the six-dimensional singularity locus of the general GoughStewart platform," Journal of Mechanical Design, vol. 128, no. 1, pp. 279-287, 2006.

[103] M. L. Husty, "An algorithm for solving the direct kinematics of general Stewart-Gough platforms," Mechanism and Machine Theory, vol. 31, no. 4, pp. 365-379, 1996. 
[104] J.-P. Merlet, "Solving the forward kinematics of a Gough-type parallel manipulator with interval analysis," International Journal of Robotics Research, vol. 23, no. 3, pp. 221-235, 2004.

[105] H. S. M. Coxeter, Introduction to Geometry, vol. 136. Wiley New York, 1969.

[106] S. Roberts, "On 3-bar motion in plane space," Proceedings of the London Mathematical Society, vol. 1, no. 1, pp. 14-23, 1875.

[107] K. Hain, Applied Kinematics. McGraw-Hill, 1967.

[108] D. Cox, J. Little, and D. OShea, Ideals, varieties, and algorithms: an introduction to computational algebraic geometry and commutative algebra. Springer Science \& Business Media, 2013.

[109] C. Xue, K.-L. Ting, and J. Wang, "Mobility criteria of planar singleloop N-bar chains with prismatic joints," Journal of Mechanisms and Robotics, vol. 3, no. 1, p. 011011, 2011.

[110] S. V. Amirinezhad, "A constraint-based approach to manipulator kinematics and singularities-MATHEMATICA files," 2019. https://drive.google.com/drive/folders/ 1bL4oyZdg9U5BV93kVHk-Qru2JPaZIHRB?usp=sharing, last accessed 2019-02-27.

[111] G. Yang, W. Chen, and I.-M. Chen, "A geometrical method for the singularity analysis of 3-RRR planar parallel robots with different actuation schemes," in Intelligent Robots and Systems, 2002. IEEE/RSJ International Conference on, vol. 3, pp. 2055-2060, IEEE, 2002.

[112] P. Wenger, D. Chablat, and M. Zein, "Degeneracy study of the forward kinematics of planar 3-RPR parallel manipulators," Journal of Mechanical Design, vol. 129, no. 12, pp. 1265-1268, 2007. 
[113] R. Willis, Principles of Mechanism. Longmans, Green, 1870.

[114] F. Buchsbaum and F. Freudenstein, "Synthesis of kinematic structure of geared kinematic chains and other mechanisms," Journal of Mechanisms, vol. 5, no. 3, pp. 357-392, 1970.

[115] C.-H. Hsu and K.-T. Lam, "A new graph representation for the automatic kinematic analysis of planetary spur-gear trains," Journal of Mechanical Design, vol. 114, no. 1, pp. 196-200, 1992.

[116] I. Talpasanu, Kinematics and Dynamics of Mechanical Systems Based on Graph-Matroid Theory. PhD thesis, The University of Texas at Arlington, 2004.

[117] L.-W. Tsai, Robot analysis: the mechanics of serial and parallel manipulators. John Wiley \& Sons, 1999.

[118] J. Schadlbauer, D. R. Walter, and M. L. Husty, "A complete kinematic analysis of the 3-RPS parallel manipulator," in 15th National Conference on Machines and Mechanisms (NaCoMM 2011), Chennai, India, Nov, 2011.

[119] D. Chablat, R. Jha, F. Rouillier, and G. Moroz, "Workspace and joint space analysis of the 3-RPS parallel robot," in ASME 2014 International Design Engineering Technical Conferences and Computers and Information in Engineering Conference, American Society of Mechanical Engineers, 2014.

[120] J.-P. Merlet, "A formal-numerical approach for robust in-workspace singularity detection," IEEE Transactions on Robotics, vol. 23, no. 3, pp. 393-402, 2007. 
[121] P. Choudhury and A. Ghosal, "Singularity and controllability analysis of parallel manipulators and closed-loop mechanisms," Mechanism and machine theory, vol. 35, no. 10, pp. 1455-1479, 2000. 